University of Louisville

ThinkIR: The University of Louisville's Institutional Repository

Electronic Theses and Dissertations

$12-2018$

\title{
CD11b regulation of $B$ cell receptor signaling in health and systemic lupus erythematosus.
}

Paul Lorenzo Dascani
University of Louisville

Follow this and additional works at: https://ir.library.louisville.edu/etd

Part of the Immunity Commons

\section{Recommended Citation}

Dascani, Paul Lorenzo, "CD11b regulation of B cell receptor signaling in health and systemic lupus erythematosus." (2018). Electronic Theses and Dissertations. Paper 3076.

https://doi.org/10.18297/etd/3076

This Doctoral Dissertation is brought to you for free and open access by ThinkIR: The University of Louisville's Institutional Repository. It has been accepted for inclusion in Electronic Theses and Dissertations by an authorized administrator of ThinkIR: The University of Louisville's Institutional Repository. This title appears here courtesy of the author, who has retained all other copyrights. For more information, please contact thinkir@louisville.edu. 


\title{
CD11B REGULATION OF B CELL RECEPTOR SIGNALING IN HEALTH AND SYSTEMIC LUPUS ERYTHEMATOSUS
}

\author{
By \\ Paul Lorenzo Dascani \\ B.S., University of Pittsburgh, 2011 \\ M.S., University of Louisville, 2015
A Dissertation
Submitted to the Faculty of the
School of Medicine
in Partial Fulfillment of the Requirements
for the Degree of \\ Doctor of Philosophy in Microbiology and Immunology \\ Department of Microbiology and Immunology \\ University of Louisville \\ Louisville, Kentucky
}

December 2018 
Copyright 2018 by Paul Lorenzo Dascani All rights reserved 

CD11B REGULATION OF B CELL RECEPTOR SIGNALING IN HEALTH AND SYSTEMIC LUPUS ERYTHEMATOSUS

\author{
By \\ Paul Lorenzo Dascani \\ B.S., University of Pittsburgh, 2008 \\ M.S., University of Louisville, 2015 \\ A Dissertation Approved on \\ December 3, 2018 \\ by the following Dissertation Committee:
}

Jun Yan, M.D., Ph.D.

Dissertation Director

Michele Kosiewicz, Ph.D.

Pascale Alard, Ph.D.

Venkatakrishna Jala, Ph.D.

David Powell, Ph.D. 


\begin{abstract}
CD11B REGULATION OF B CELL RECEPTOR SIGNALING IN HEALTH AND SYSTEMIC LUPUS ERYTHEMATOSUS

Paul Dascani
\end{abstract}

December 3, 2018

Loss of function mutation in CD11b has been associated with incidence of systemic lupus erythematosus (SLE), a disease driven by B cell production of pathogenic autoimmune antibody. Our previous work has revealed the ability of CD11b to regulate B cell receptor (BCR) signaling and control autoimmune disease in mice. However, how CD11b regulates the immune response under normal conditions remains unknown. Through the use of a CD11b knockout model, we demonstrated that CD11b-deficient mice have an elevated antigen-specific humoral response in a Th2 type immunization. Deletion of CD11b resulted in elevated serum IgM and IgG antibody, increases in antigen-specific germinal center (GC) B cells and plasma cells (PCs), and increased expression of survival and regulatory factors Bcl-XL and Blimp1 within these subsets. Subsequent experiments using a tissue-specific CD11b deletion model revealed this effect to be B cell intrinsic, and not altered by myeloid cell CD11b expression. Examination of BCR signaling in GC B cells of CD11b knockouts revealed defects in association of negative regulators SHP-1 and pLyn with the BCR following stimulation, as well as alterations in nuclear 
localization of GC regulators cMyc and FOXO1. Through the use of a CD11b-reporter mouse model, we identified multiple novel CD11b-expressing B cell subsets that are dynamically altered during immunization and autoimmune conditions. Lastly, we investigated the incidence of CD11b mutation rs1143679 in SLE patients and examined its effect on the clinical outcome of disease and impact on B cell function. These studies describe a novel role for $\mathrm{CD} 11 \mathrm{~b}$ in regulation of the healthy humoral response at the GC stage and reveal previously unknown populations of CD11b expressing B cell subsets, suggesting a complex function for $\mathrm{CD} 11 \mathrm{~b}$ in $\mathrm{B}$ cells during development and maturation. 
TABLE OF CONTENTS

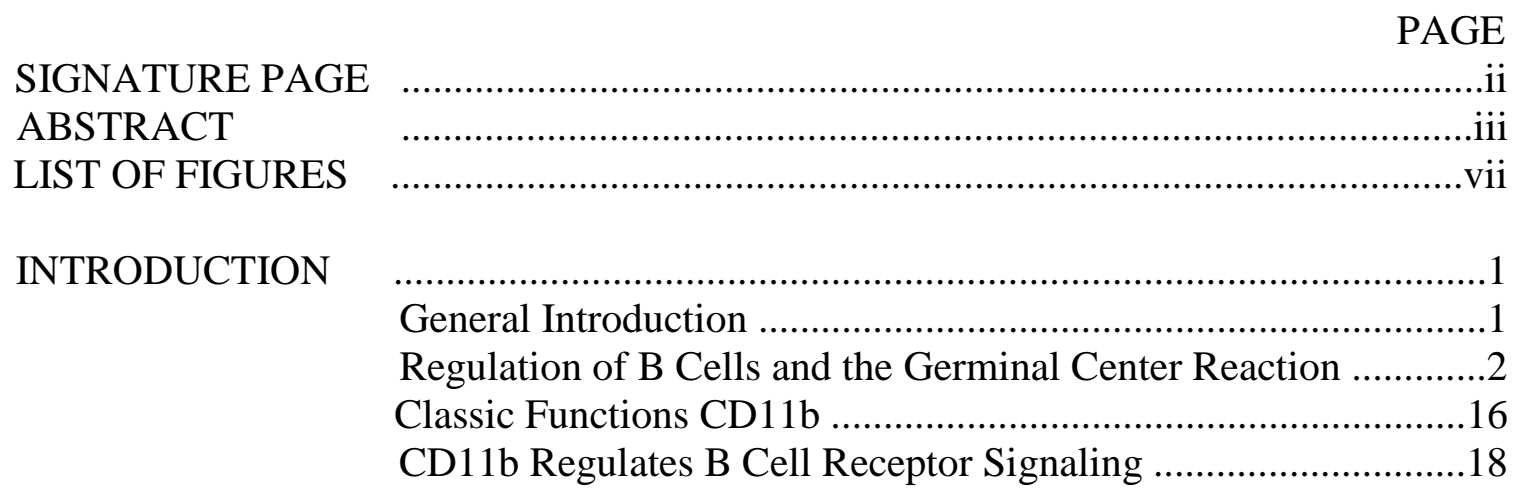

CHAPTER I: CD11B REGULATION OF B CELL RECEPTOR SIGNALING IN

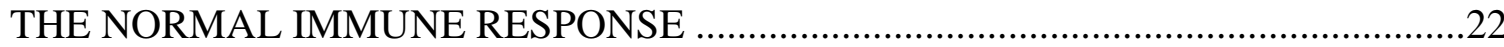

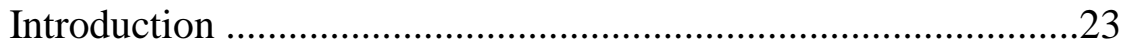

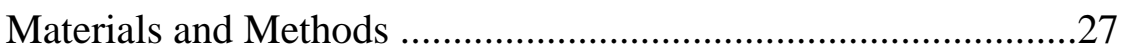

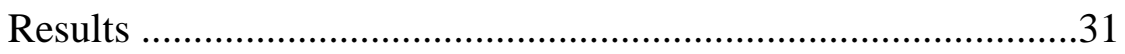

Global CD11b Deficiency Alters Antibody Production and GC

Formation in Non-autoimmune Mice ............................................31

CD11b Regulates Germinal Center B Cell BCR Signaling ..........37

B Intrinsic CD11b Regulates the Th2 Antibody Response .........45

Myeloid Cell Loss of CD11b Does Not Impact the Th2

Antibody Response.......................................50

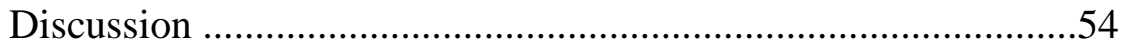

CHAPTER II: DYNAMIC EXPRESSION OF CD11B ON B CELLS IN NAÏVE

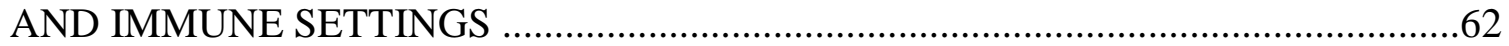

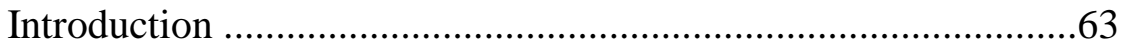

Materials and Methods .............................................................67

Results.................................................... 70

B cell CD11b Expression is Altered in a Th2 Immunization

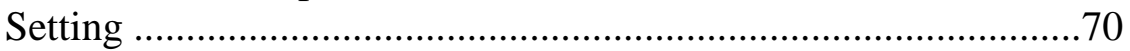

Progression of Lupus-Like Disease in Mice Impacts B Cell

Expression of CD11b ............................................................. 81

Comparison of the CD11b Expressing Plasma Cell Repertoire ...87

Discussion ....................................................................................

CHAPTER III: THE IMPACT OF CD11B POLYMORPHISM ON B CELLS IN SYSTEMIC LUPUS ERYTHEMATOSUS.........................................................96

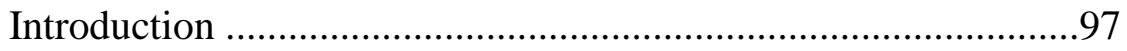

Materials and Methods ...............................................................106 
Results

A Phenotypic Survey of ITGAM Mutation rs1143679 in SLE

Patients of the Louisville Region

Discussion

CONCLUSIONARY REMARKS AND FUTURE PERSPECTIVES

\section{CHAPTER IV: TRASNCRIPTION FACTOR STAT3 SERVES AS A NEGATIVE REGULATOR CONTROLLING IGE CLASS SWITCHING ..................................123

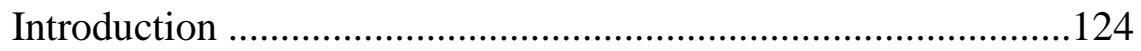 \\ Materials and Methods .......................................................132

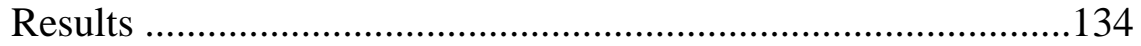

STAT3 Deficiency in B and T Cell Subsets Disrupts the

Regulation of Class Switching in a Th2-type Immunization ......137

STAT3-Dependent Regulation of Germinal Center Class

Switching is a B cell Intrinsic effect ........................................142

Depletion of STAT3 Regulates Germinal Center Class Switching

In Vitro .151

Target Genes of STAT3 in the GC B Cells ..............................156 STAT3 in B Cells Regulates Lung Inflammation During Inhaled Allergy Reactions 160

Discussion .164

REFERENCES

CURRICULUM

VITAE 


\section{LIST OF FIGURES}

Figure 1: Increased antibody response in CD11b-knockout mice following Immunization 33

Figure 2: Germinal center B cells are increased in CD11b-knockout mice 34

Figure 3: Plasma cells are increased in CD11b-knockout mice ………….........................35

Figure 4: CD11b deficiency alters GC and PC regulator expression ..............................36

Figure 5: Loss of CD11b alters SHP-1 colocalization with the BCR ..............................39

Figure 6: Loss of CD11b alters pLyn colocalization with the BCR ................................40

Figure 7: Loss of CD11b alters pSyk colocalization with the BCR ................................41

Figure 8: CD11b deficiency alters pSyk colocalization in non-GC B cells ....................42

Figure 9: Loss of CD11b alters cMyc Nuclear Translocation ........................................43

Figure 10: Loss of CD11b alters FOXO1 Nuclear Translocation ....................................44

Figure 11: Generation of CD11b-loxp mice …………...............................................46

Figure 12: B Cell loss of CD11b leads to increased serum antibody ...............................47

Figure 13: The effect of B cell CD11b deletion on GC and PC formation ......................48

Figure 14: The effect of CD11b deletion on follicular, marginal zone, and memory B cells

Figure 15: Efficiency of Lyz2-cre driven deletion of CD11b B ......................................51

Figure 16: Myeloid cell loss of CD11b does not alter the antibody response ..................52

Figure 17: Myeloid cell loss of CD11b does not alter GC and PC differentiation ............53

Figure 18: Loss of CD11b alters GC B cell signaling and regulation and enhances the

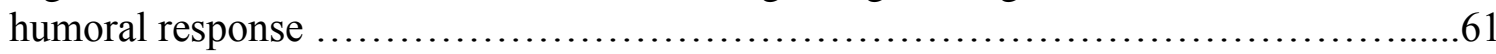


Figure 19: Generation of CD11b-reporter mice .73

Figure 20: The impact of immunization on CD11b expression in early-stage developing B cells of the bone marrow

Figure 21: The impact of immunization on CD11b expression in later-stage developing B cells of the bone marrow .75

Figure 22: The impact of immunization on CD11b expression in splenic marginal zone and follicular B cells .76

Figure 23: The impact of immunization on CD11b expression in splenic GC B cells .....77

Figure 24: The impact of immunization on CD11b expression in splenic memory B cells .78

Figure 25 The impact of immunization on CD11b expression in splenic plasma cells ....79 Figure 26: The impact of immunization on CD11b expression in long lived bone marrow plasma cells .80

Figure 27: Progression of autoimmune disease in FRT-LPR mice .83

Figure $28 \mathrm{Fas}^{\mathrm{lpr}}$ autoimmune disease impacts splenic B cell CD11b expression .............84

Figure 29: Fas ${ }^{\mathrm{lpr}}$ autoimmune disease impacts plasma cell CD11b expression ................85

Figure 30: Fas ${ }^{\mathrm{lpr}}$ autoimmune disease impacts developmental B cell CD11b expression.86

Figure 31: Progression of autoimmune disease in FRT-ABIN1 mice .............................89

Figure 32: FRT-ABIN1 plasma cell CD11b expression .................................................90

Figure 33: CD11b is dynamically expressed across different $\mathrm{B}$ cell subsets ..................93

Figure 34: Incidence of rs1143679 in Louisville, KY area SLE Patients .......................111

Figure 35: Clinical and demographic associations of rs1143679 in SLE ......................112

Figure 36: Identification of peripheral B cell subsets in SLE patients ..........................113

Figure 37: Peripheral B cell subsets in SLE patients with ITGAM mutation ................114

Figure 38: In vitro differentiation of plasmablasts from SLE patient PBMCs ................115

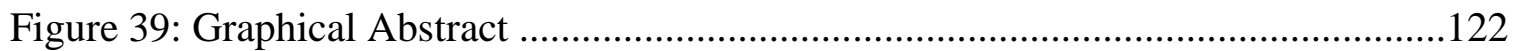


Figure 40: STAT3 regulates IgE antibody class switching in a Th2-type response 139

Figure 41: STAT3 regulates GC and PC IgE class switching in a Th2-type response ...140

Figure 42: Loss of STAT3 leads to deceased antibody affinity in CD2-Cre driven STAT3 knockouts

Figure 43: STAT3 regulation of class switching is a B cell intrinsic effect 146

Figure 44: B cell STAT3 depletion alters antibody affinity 146

Figure 45: Loss of STAT3 in B cells alters early germinal center and plasma cell IgE class switching

Figure 46: STAT3 regulates germinal center and plasma cell IgE class switching in B cells

Figure 47: STAT3 depletion alters germinal center light zone and dark zone Distribution 149

Figure 48: B cell deletion of STAT3 alters germinal center gene expression 150

Figure 49: STAT3 regulates IgE class switching of in-vitro generated germinal center B cells 153

Figure 50: STAT3 regulates IgE class switching of in-vitro generated germinal center B cells

Figure 51: STAT3 regulates germinal center related genes in in vitro generated germinal center B cells .155

Figure 52: STAT3 binding patterns in GC B cells 157

Figure 53: Gene Ontology (GO) analysis of differentially expressed peak related genes in STAT3 control and cKO GC B cells 158

Figure 54: STAT3-bound genes in GC B cells 159

Figure 55: Generation of an asthma-like model in STAT3 deficient mice 161

Figure 56: STAT3 deficiency causes increased inflammation in an asthma-like model.162

Figure 57: STAT3 deficiency causes increased eosinophil infiltration in an asthma-like model 


\section{INTRODUCTION}

Maintaining an effective defense from outside harm is crucial to an organism's survival, at both the macro and micro level. Infection from foreign bacteria, viruses, and other parasites all pose a deadly threat, but the mammalian immune system has evolved to combat these pathogens.

Two major forms of immunity function to combat these dangers are; innate and adaptive immunity (1). The innate immune system is characterized by cells which initiate a response based on a non-variable, germ-line encoded receptor that recognizes broad pathogen-associated molecular patterns (PAMPs) (2). The adaptive arm of the immune system is comprised of cells which contain highly specific receptors generated through somatic recombination of the germ-line, and are able to produce immune memory; allowing a greater, more rapid, and more effective response upon repeated encounter with a given antigen (3).

The adaptive compartment is composed of two broad cell types: T cells and B cells. The T cell compartment is largely considered to propagate cell-mediated immunity; driving elimination of pathogenic antigen by direct cell-to-cell contact and recognition via the T-cell receptor (TCR) (4). Conversely, B cells are mediators of humoral immunity. Not requiring direct cell contact with the B cell receptor (BCR), B cells secrete antibodies which are able to bind their cognate antigen at sites distal from the B cell itself. This binding action assists in pathogen elimination by activation of the 
complement system, enhancing recognition of the foreign body by innate cells, and modulating the $\mathrm{T}$ cell response (5-7).

Activation of the B cell antibody response is a tightly regulated process. Inproper regulation can cause the production of antibody against self-antigens and lead to autoimmunity. Overzealous regulation would leave the B cell response inert, unable to effectively defend the body from foreign pathogen. An intricate network of signals and mechanisms act to maintain a healthy level of B cell response and strike a balance between necessary and unnecessary activation of B cells. In this work, we will discuss the role of cluster of differentiation $11 \mathrm{~b}(\mathrm{CD} 11 \mathrm{~b})$ as a novel regulator of $\mathrm{B}$ cell activation in the normal B cell response, as well as its implications in autoimmunity. We will also investigate the impact of CD11b mutation on B cell function in systemic lupus erythematosus (SLE), a disease characterized by a severe pathogenic autoimmune B cell response.

\section{Regulation of B Cells and the Germinal Center Reaction}

During development, B cells undergo random V(D)J gene recombination of the B cell receptor in order to produce a wide diversity of antigen specificities. The earliest B cell developmental stage to feature a functional, V(D)J rearranged Ig sequence is the immature B cell stage in the bone marrow. Even in healthy human subjects, $75 \%$ of the clones produced from this population were found to be autoreactive. This included major epitopes such as insulin, LPS, and nuclear antigens $(8,9)$. To eliminate the potential damage of these cells, several mechanisms exist to induce B cell tolerance, such as negative selection/deletion, induction of anergy, and re-editing of the BCR (10). Selfreactive $\mathrm{B}$ cells may also escape central mechanisms of selection and appear in the 
periphery, only to remain inactive even in the presence of self-antigen. These cells are classified as "ignorant" or "indifferent to self-antigens (11). Peripheral autoreactive B cells may also be regulated by selective differentiation into the marginal zone B cell compartment, which is more easily regulated by dependence on $\mathrm{T}$ cell interaction (12). The prevalence of these regulatory mechanisms at every B cell stage clearly indicates the importance of preventing the production and activation of autoreactive B cells.

B cells are able to sense nucleic acids via TLR7 and TLR9, which recognize RNA and DNA, respectively. The receptors, located intracellularly in the endosome, assist in the recognition of and response to microbes via detection of foreign nuclear antigen (13). However, they are also sensitive to self-nuclear antigen, and are involved in production of anti-nuclear antibodies (ANAs) (14). Cell death leads to release of complex nuclear antigens, such as Smith antigen/ribonucleoprotein (Sm/RNP) and nucleosomes. When autoreactive B cells specific for these epitopes encounter these free-floating antigens, they engage the BCR and are translocated to the endosomal compartment. Here, they can interact with TLR7 and TLR9, which serve as co-stimulatory signals to enhance the activation cascade which results in the B cell production of ANAs (15). Indeed, impaired TLR7 and TLR9 activity was shown to reduce production of autoantibody against Sm/RNP and DNA in mouse models of lupus, while enhanced TLR7 expression was able to induce lupus-like disease in healthy mice and accelerate pathogenesis in lupus-prone strains $(16,17)$. In humans, SNPs leading to increased TLR7 expression have been associated with SLE in certain populations (18). These findings demonstrate a necessity for control of B cell activation via the innate RNA and DNA-sensing pathway. 
Type-1 Interferons (IFN I) are responsible for activation signaling in the innate and adaptive immune system. These pathways control the presentation of antigen and production of cytokines vital to B cell activation. In B cells, IFN I also activates expression of BAFF, which is an essential cytokine for differentiation and development (19). Plasmacytoid dendritic cells (pDCs) are a major producer of IFN I, which then provides necessary activation signals for B cells. Overactivation of pDCs can lead to overproduction of IFN I and subsequently, overactivation of B cells (20).

The BCR signaling cascade is one of the major mechanisms for controlling B cell activation. Disruption of these mechanisms can lead to unwanted activation of the B cell response and potentially autoimmunity. A large cohort of SLE-associated genes are involved in this pathway, such as scaffold adaptor protein BANK1. BANK1 amplifies BCR signaling by linking the BCR-induced activation of Src-family kinases, including LYN, with PLC $\gamma 2$ cascade IP3 and DAG calcium influx signaling (21). This mechanism then leads to further downstream activation of the PRKCB gene, which is also strongly associated with SLE (22). Indeed, recent mouse-model studies demonstrated BANK1deficient mice have decreased $\mathrm{IgG}$ production during autoimmune disease development (23). Another important player in the BCR pathway is RasGRP3. This protein amplifies Ras-mediated MAPK signaling and is involved in B cell antibody production and proliferation (24). It is also expressed in other cell types critical to the immune response, such as T cells and myeloid cells (25). Dysfunction of RasGRP3 in B cells can drive autoimmune activation in SLE and may contribute to disease progression via other cell types as well. 
The Src-family kinases LYN, BLK, and CSK serve as BCR inhibitory signals, and defects in these molecules have been linked to development of autoimmune disease. LYN-deficient mice have been shown in several models to develop B cell hyperactivity and autoimmunity (26). LYN enacts inhibition by recruitment and activation of inhibitory surface receptors FcGR2B and CD22. The cytoplasmic tail domains of molecules then recruit and activate SHIP and SHP-1 phosphatases which reduce the strength of the B cell activation signal (27). BLK is able to promote interaction between the aforementioned BANK1 and PLC $\gamma 2$. Reduced BLK expression has been correlated with reduced frequency of marginal zone B cells and pre-B cells, however, these fewer marginal zone cells are hyper-responsive to BCR stimulation (28). BLK reduction also enhanced cytokine production and kidney disease in Fas ${ }^{\mathrm{lpr}}$ lupus-prone mice (29). Lastly, CSK directly interacts with intracellular phosphatase LYP; which regulates activation of LYN. In SLE patients, overexpression of CSK has been associated with increased IgM autoantibody production and doubled frequency of transitional B cells. This CSK-LYPLYN mechanism affects SLE pathogenesis at multiple stages of B cell maturation (30, 31). Protein tyrosine phosphatase non-receptor type 22 (PTPN22) is associated with many autoimmune disorders, including SLE. Similar effects have also been confirmed in several mouse models $(32,33)$. In B cells as well as T cells, PTPN22 regulates CSK to control the BCR or T cell receptor (TCR) activation threshold (34). The interaction of PTPN22, LYN, BLK, and CSK in controlling BCR ligation signal is a crucial area of study for understanding the regulation of B cell activation.

Lipid phosphatase SHIP-1 and non-receptor tyrosine phosphatases SHP-1 and LYB/PEP are regulators of B cell tolerances, and have been implicated in autoimmune 
disease (35). In mice, induced loss of SHIP-1 or SHP-1 caused a reversal of anergic state in nuclear-antigen reactive B cells, eventually leading to differentiation into plasma cells and high ANA production (35). Other studies have shown development of lupus-like disease in SHIP-1 or SHP-1 deficient mice $(36,37)$. These results not only elucidate SHIP-1 and SHP-1 as vital controllers of B cell activation, but also show the reversibility of anergy and the roles this process may play in autoimmune disease.

There are multiple co-receptors that inhibit B cell activation via recruitment of SHP-1, such as CD22, CD32B, and CD $(35,38)$. However, CD72 is uniquely specified in maintaining tolerance to nuclear antigens, making it a prime target for study in ANAdependent SLE. Additionally, polymorphisms in CD72 have been found to associate with SLE susceptibility (39). CD72 selectively binds Sm/RNP, and acts to inhibit activation signals when the BCR encounters and binds Sm/RNP antigen (40). This divalent binding of colocalized BCR and CD27 causes BCR-associated Lyn to phosphorylate the cytoplasmic tail of CD72, which in turn activates SHP-1 to suppress the BCR signal (41). In mice, deletion of CD72 causes severe lupus-like disease, even more drastically than disease caused in deletion of other inhibitory receptor CD22 (42). Interestingly, CD72 does not repress BCR signal stimulated with polyclonal anti-IgM antibody, which further enforces the highly-specific function of this repressor in maintaining tolerance to Sm/RNP nuclear antigen (43). CD72's high specificity also prevents it from binding microbial RNA, allowing it to "filter out" self-RNA from being sensed downstream by TLR7 (40). This finding implicates the possibility for other common auto-antigen restricted suppressive co-receptors in B cell signaling. 
Self-reactive and poly-reactive B cells that avoid negative selection and deletion may accumulate in the B-1 B cell fraction. B-1 cells tend to be polyreactive $\operatorname{IgM}$ or IgA class, and are able to form independent of $\mathrm{T}$ cell help via multivalent antigens such as LPS (44). This fraction can be further divided into B-1a and B-1b subsets. B-1a cells produce naturally occurring low affinity autoantibodies that are useful in the clearance of apoptosis-derived debris (45). The B-1b fraction generates IgM memory cells in response to $\mathrm{T}$-independent antigen and has been shown to prevent certain secondary infections (46). The expansion of these B-1 cells has been correlated with autoimmune disorders, including SLE, in humans and mice (47). Deletion of SHP-1 in mice causes lupus-like disease and expansion of B-1 cells. However, deletion of Siglec-10, a SHP-1 recruiter expressed highly in B-1 cells, caused expansion of B-1 cells but only minor autoimmune symptoms. Though SHP-1 is an important regulator of B-1 cell expansion, their enhanced response in its absence is not potent enough to induce severe lupus-like autoimmunity alone $(37,48)$. This demonstrates the dynamic nature of $\mathrm{B}$ cell regulation, outlining the need for different levels of control depending on the specific subset's function and potency.

Not all B cell subsets are activators of the humoral response. Regulatory B cells are able to suppress the function of $\mathrm{T}$ lymphocytes though multiple mechanisms, including IL-10 (49). This, in turn, is able to regulate the B cell activation via reduced T helper cell interaction, resulting in a reduced antibody response. Not limited to the adaptive arm, regulatory B cells can also inhibit DCs, NK cells, and macrophages, which can contribute to an overall limited inflammatory response (50). Indeed, animals lacking IL-10 producing regulatory B cells develop more severe autoimmune disease, mediated 
by several cell types (49). Interestingly, this regulatory subset is able to differentiate into antibody producing plasma cells after a brief period of IL-10 production, revealing a mechanism of transient regulation by B cells on their own response (51).

The interaction between $\mathrm{B}$ cells and $\mathrm{T}$ cell is an important step in immune activation. B cells can serve as antigen presenting cells (APCs) by endocytosing autoantigens and presenting them mostly on MHC-II (52). Signaling via the MHC-II is heavily regulated by several associative molecules including CD19 and CD22 via SHP-1, drawing many parallels with BCR signal regulation (53). MHC-II genes HLA-DR2 and HLA-DR3 are strongly associated with SLE, though their exact role is not well understood (54). Co-stimulatory signals also play a large role in regulation of B cell activation. B cell OX40L interacts with OX40 (CD134) on T cells to enhance B cell proliferation and differentiation. In T-regulatory cells, this interaction negatively regulates generation of IL-10, which helps maintain tolerance (55).

The marginal zone (MZ) B cell compartment differentiates from transitional B cells; a brief stage of development following generation in the bone marrow but before maturity in the spleen. Named for their location in the marginal zone of the spleen, MZ B cells act as an early defense against T-independent blood-borne pathogens (56). They respond strongly to TLR activation and are able to quickly differentiate into plasma cells without the need for direct co-stimulation signals from $\mathrm{T}$ cells, instead using soluble signals and cytokine from DCs, neutrophils, and T cells (57). In humans, MZ B cells are present in the spleen and peripheral blood, but in mice they are restricted to the MZ of the spleen (58). Activation of MZ B cells is controlled by BCR, BAFF/BAFF-R, and Notch signaling. Their response is controlled by standard BCR negative regulatory mechanisms 
such as SHP-1 and CD22 inhibition. MZ B cell expansion has been observed in multiple lupus mouse models and have also displayed increased generation of anti-dsDNA IgM antibody $(59,60)$. In humans however, the role of MZ B cells remains unclear.

Follicular (FO) B cells also derive from the transitional B cell stage. Unlike MZ B cells, FO B cells do require cognate T cell signaling help which causes them to be much slower to differentiate into effector plasma cells. Thus, their role in the T-independent is minimal (56). However, they are the major source of the T-dependent B cell reaction. FO B cells migrate between the splenic follicles, lymph nodes, and the circulation until they contact antigen. They may also encounter antigen presented by MZ B cells in the follicle (61). Upon activation, they migrate to the B-T border of the lymph node and spleen via CCR7 expression. Here they engage with T helper cells and receive proliferative signals. The specific of this interaction vary depending on the type of T helper cell (Th1, Th2, Th17, Tfh), which is dependent on T cell-DC interaction the antigen/pathogen molecular pattern (62). Following activation, FO B cells differentiate into extrafollicular plasma cells, or enter the germinal center reaction to increase antigen affinity (63).

To produce high amounts of effector antibody, including autoreactive epitopes, activated B cells differentiate into plasma cells. This process may happen either through the extrafollicular pathway, or though the germinal center (GC) reaction. Following engagement of mature B cells with cognate antigen, B cells migrate to the T-B follicle border in secondary lymphoid organs to receive CD4 T cell co-stimulatory signals. These signals drive rapid proliferation of the B cells which then locate to either the outer follicle pathway at the T-B border to become extrafollicular plasma cells (EF PCs), or to the central B cell follicle to initiate the germinal center. The exact mechanisms controlling 
this divergence are still not fully understood, though some studies have suggested BCR affinity may be a factor under competitive conditions (63).

EF PCs produce a faster but lower affinity antibody response which is important in quickly responding to infection (64). These differentiating B cells increase expression of Blimp-1 (or PRDM1) as well as CXCR4 and CD138 (65). T-helper cells directly interact with the B cells and provide essential stimulatory signaling through CD40, ICOS, and IL-21 (66). This drives differentiation into EF PCs and class switch recombination (CSR) of the Ig heavy chain (63). These cells then chemotaxis via CXCR4 to the red pulp of the spleen and form EF foci. Here, higher affinity BCRs are selected to survive and proliferate. These newly formed EF PCs can then produce an antibody response and reside in peripheral lymphoid organs, as well as generate memory B cells $(67,68)$. EF PCs may play a role in autoimmunity due to their dependence on TLR9 signaling to migrate to the EF foci, which would encourage differentiation of DNA-reactive B cells (69). However, most research is focused on the GC pathway of PC differentiation rather than the shorter lived and lower affinity response of EF PCs. There are also no effective markers to distinguish EF PCs from GC PCs, making their effect hard to delineate in long-term responses (70). Recently, some animal studies have found enhanced EF PC differentiation in autoimmune models, and a possible large population of EF derived PCs in SLE patients (71). Though their role is less characterized in SLE, the EF PC population may play a role in disease progression and warrants further investigation. Cells that do not enter the EF pathway instead enter the GC reaction inside the central B cell follicle. Expression of transcription factor B cell lymphoma 6 (Bcl6), an essential and hallmark regular of the GC, begins at this time and continues for the 
majority of the GC stage (72). Cells in the center may cluster together and interact with follicular dendritic cells (FDCs) to initiate the GC reaction $(73,74)$. FDCs present in these tissues help to organize B cells to initialize and form the GC structure, serve as antigen presenting cells, and propagate B cell survival and proliferation during the GC reaction $(75,76)$. Infection or immunization is required to initiate $\mathrm{GC}$ formation in healthy individuals, but lupus mouse models are able to spontaneously generate GCs, indicating an important role for this process in disease (77).

The mature GC is defined by two distinct and polarized compartments; the light zone (LZ) and the dark zone (DZ). The DZ forms more closely to the bordering T cell follicle and contains very little supportive FDCs. Here, the B cells cluster and undergo rapid proliferation. These cells are hallmarked by expression of chemokine CXCR4 and activation-induced cytidine deaminase (AID)(AICDA), as well as near-constant activity of the $\mathrm{G} 2$ and $\mathrm{M}$ phases of proliferation $(78)(78,79)$. AID expression combined with high-error DNA polymerase drives B cell somatic hypermutation (SHM), which causes random rearrangement of the Ig that may possibly increase antigen affinity (80). Every round of division further increases the amount of mutations and thus the chance of increased (and perhaps decreased) affinity maturation. These DZ GC B cells will then migrate to the $\mathrm{LZ}$ to compete for selection with their newly re-arranged BCR. The highest affinity B cells will out-compete those of lower affinity and return to the DZ for further rounds of SHM and CSR. It has been demonstrated that many more cells move from the DZ to the $\mathrm{LZ}$ than from the $\mathrm{LZ}$ to the $\mathrm{DZ}$, indicating that high amounts of selection are occurring in the LZ paradigm of this process (81). 
The LZ forms close to the marginal zone and contains many FDCs, as well as non-participant naïve B cells $(82,83)$. FDCs present antigen to B cells via the Fc $\gamma$ receptor and provide anti-apoptotic signal via BAFF and Notch ligands. These interactions are short-lived, lasting about 3 minutes $(84,85)$. This step serves a selector for high affinity BCRS, as lower affinity B cells will have weaker and more transient interactions with FDCs, depriving them of necessary anti-apoptotic signals. Defective negative regulation of the BCR at this step can allow for the survival of B cells that have weak binding or off-target specificity, including self-antigens. FDCs can also drive offtarget effects through the presentation of self-antigen for selection. Indeed, a study has identified the presence of nuclear antigens presented on FDCs on SLE patients (86). Some SLE patients also display disarray of the FDC network that associates with lymphadenopathy (87). These findings, supported by the essential role of FDCs in some mouse models of arthritis, suggest FDCs may play a part in regulation of the autoreactive B cell response (88).

The LZ also contains $\mathrm{T}$ follicular helper (Tfh) cells, which provide crucial signals to B cells to regulate their size, restrict low affinity GC B cell entry into the reaction, provide support signals for high affinity GC B cells, and select for high-affinity B cell affinity maturation $(89,90)$. Like GC B cells, Tfh cells are also dependent on Bcl6 expression during the GC reaction (72). Tfh engagement is vital as GC B cells cannot trigger BCR engagement and must depend on Tfh signaling to promote proliferation and survival. CD40L, IL-4, and IL-21 are the most critical signals provided by Tfh cells, though there are others (91). IL-4 and IL-21 promote class switching to $\operatorname{IgG}_{1}$, while CD40 is a vital co-activation signal of the BCR (92). These signals compete against Fas- 
FasL interactions that can lead to cell death, thereby serving as another layer of selection for higher-affinity BCRs (91). This Fas-FasL signaling is also important in the prevention of autoimmunity, as B cell depletion of Fas leads to lupus-like disease (93). Interestingly, the Tfh cells receive many inhibitory signals from GC B cells to prevent over proliferation due to constant engagement of the TCR. High PD-L1 expression on GC B serves not only to inhibit Tfh proliferation, but also reduces Tfh ICOS and IL-21 expression, thereby self-regulating the amount of Tfh help the GC B cell receives (94). This intricate interaction with Tfh cells acts as a crucial regulatory step of the GC reaction, and its disruption may have pathogenic effects. Increased frequency of Tfh cells in the periphery has been associated with autoantibody-related diseases, including SLE (95). Indeed, clusters of Tfh and GC B cells are found in the kidneys of lupus nephritis patients, demonstrating a direct link to B cell regulation and pathogenesis (96).

The GC reaction gives rise to both memory B cells and PCs. Lower affinity GC B cells differentiate into the memory subset, while higher affinity cells become PCs. Memory cells also arise from the GC much earlier than PCs $(97,98)$. IL-21 signaling also drives differentiation to PCs over memory B cells (99). At the transcriptional level, Bach2 has been shown to be important in memory B cell selection, while other transcription factors such as Blimp1 and IRF4 are tied to the PC fate $(100,101)$. PC differentiation is initiated in the LZ, but the cells then move towards the dark zone to complete their final rounds of proliferation as plasmablasts before exiting the GC into the red pulp (102). Proper regulation of these PC and memory B cell differentiation processes is vital to the formation of a strong humoral response, and can have pathological consequences (103) 
Newly differentiated PCs usually survive for a few weeks without further stimulation, decreasing by as much as $95 \%$ by 30 -days post infection in some models (104). Memory B cells are traditionally considered the mediators of long-term humoral immunity, but s subset of long-lived PCs has also been identified. These cells have been found as late as 10 years post-vaccination and persist despite depletion of memory B cells $(105,106)$. Long-lived PCs contribute to the pathogenesis of lupus-like disease in mice and are unaffected by some traditional treatment methods $(107,108)$. The survival of both long and short-lived PCs is dependent on APRIL and BAFF-signaling and is enhanced by other signals such as IL-6 and CD28 (109-111). The prolonged survival of PCs in SLE is one cause for high autoantibody levels and subsequent tissue damage. Lupus mouse models show increased expression of APRIL, BAFF, and IL-6, and these trends can be found in human SLE patients as well $(112,113)$. Under normal conditions, limited amounts of PCs can be found in peripheral organs such as the spleen, bone marrow, and even kidney. In lupus mouse models, the amount of PCs in these sites is greatly increased, indicating an increase in PC support and survival signals in these tissues (114).

PC tolerance is maintained through different mechanisms, depending on the source of the cells. EF PCs are tolerogenic in nature due to the short duration of their response, and the decreased affinity compared to GC PCs. Also, most EF PCs produce IgM class antibody rather than more potent and pathogenic IgG. In MRL-lpr lupus mice, the presence of $\operatorname{IgM}$ autoantibody was able to delay onset of disease, demonstrating a role for antibody class balance in maintenance of tolerance (115). 
GC PCs are selected for tolerance in the GC phase. One proposed mechanism is through competition for T-helper or FDC survival signal. GC B cells which begin as or become self-reactive through SHM will be outcompeted for survival signaling by cells that remain on target for foreign antigen and either die or fail to differentiate (78). Though this mechanism would fail if T-helper or FDCs first bind and present selfantigens, it does offer a layer of protection against autoimmune activation. Interestingly, the frequency of IgG class self-reactive memory B cells is much higher than IgG PCs, indicating a role of self-reactivity in determining GC B cells differentiation between these two subsets (116). Prolonged expression of MHC-II on GC PCs though the proliferative stage suggests another tolerance mechanism in the requirement for $\mathrm{T}$ cell interaction as they reach terminal differentiation (117).

Hyper responsiveness of B cells can result from ineffective regulation. Increased signaling in the BCR activation pathway following antigen binding cascades downstream to increase the $\mathrm{B}$ cell response. There are multiple signaling molecules and factors involved in this mechanism, many of which are established genetic risk alleles for SLE (118). This includes both increases of function for pro-signaling molecules such as BANK1 as well as decreased function for negative regulators of BCR signaling including LYN $(119,120)$. Non-BCR activation pathways, such as type-I IFN, can also contribute to hyperresponsiveness by decreasing the BCR signal threshold required to induce the $\mathrm{B}$ cell response (121). The hyperresponsiveness caused by these abnormalities can allow autoreactive B cells to circumvent affinity-based selection mechanisms in the LZ of the GC, leading to proliferation in the DZ and eventual production of PCs (122). TLR signaling also contributes to B cell activation. Increased TLR7 expression in mice has 
been shown to spontaneously increase GC formation and plasmablast production leading to autoimmunity (123).

All of these findings clearly outline a widespread and complex pathway of B cell development and regulation. Multiple mechanisms contribute to maintaining an appropriate level of B cell response. Dysfunction of these mechanisms can lead to overactivation and autoimmune disease. Not limited to the B cells alone; abnormalities in the various cells that support and interact with B cells can also drive this hyperresponsiveness.

\section{Classic Functions of CD11b}

$\mathrm{CD} 11 \mathrm{~b}$ is a surface-bound receptor classically associated with myeloid cells such as neutrophils and macrophages. An association of CD11b mutation with SLE incidence was identified several years ago, though much of its disease contribution was attributed to typical CD11b-expressing myeloid cells (124). However, the B cell centric nature of SLE begs the question; how does CD11b impact B cells in disease pathogenesis? Does it act indirectly via alterations in myeloid cell function, or do these mutations affect B cell function directly? A better understanding of the classic function of CD11b is necessary to approach this question.

CD11b is expressed on phagocytic cells, natural killer (NK) cells, and has also been found on small populations of $\mathrm{T}$ and $\mathrm{B}$ cells. It makes up the $\alpha$ subunit of the heterodimer Mac-1, also known as CR3 or integrin $\alpha_{M} \beta_{2}$ (ITGAM). The other half of the integrin, the $\beta$ subunit, is CD18. Mac- 1 is a member of the $\beta_{2}$ integrin family expressed exclusively on leukocytes, which includes LFA-1, p150, and integrin $\alpha_{D} \beta_{2}$. The $\alpha$ portions of all family members are similar in both structure and function (125). 
Mac-1/CR3 can act as a complement receptor, cellular adhesion molecule, and a transmembrane signaling adapter. In phagocytes and NK cells, it can bind ICAM-1 on endothelial cells to allow entry into sites of inflammation (126). The ability to bind complement is the source of its CR3 namesake. This mechanism is used in initiating phagocytosis of microorganisms, apoptotic cells, or cellular debris opsonized with iC $3 b$ or immune complexes (127). However, it is also capable of binding many other ligands and is regarded as loosely restricted.

Mac-1 signaling is bi-directional, capable of both inside-out and outside-in pathways (128). Inside-out pathways are initiated via intracellular signaling from other nearby surface receptors like the TLRs, chemokine receptors, and the TCR. These induce conformational changes in Mac-1 that can alter its affinity for ligands. Outside-in signaling is initiated by direct binding of Mac-1 with extracellular ligands, before or after inside-out signaling has already taken place. These pathways are involved in phagocytosis, cellular adhesion, changes in gene expression, and other functions (129).

In DCs, Mac-1 can inhibit function at inflammatory sites that lack pathogenic signals. This downregulatory mechanism decreases DC expression of maturation marker CD86 to dampen their ability to stimulate $\mathrm{T}$ cell proliferation to reduce unwanted immune responses in a non-pathogen inflammatory event (130). Macrophage Mac-1 is able to negatively regulate low-avidity TLR inflammatory responses. In a negativefeedback pathway, TLR-induced PI3-kinase activates CD11b to recruit Srk-family kinases that degrade signaling downstream of TLR, such as MyD88 and TRIF (131). Another macrophage function of Mac-1 is cross-regulation of heterologous receptor signaling. It can regulate type-I interferon and TLR4 signaling via Srk-family kinases that 
act as inhibitors of downstream signaling while simultaneously inducing expression of anti-inflammatory cytokine IL-10 (132). Mac-1 acts as a negative regulator in NK cells as well. NKs greatly increase expression of CD11b upon maturity to keep unwanted activation in check. In an inducible hepatitis model, NK CD11b deficiency led to much more rapid and severe liver damage, thus demonstrating the pathogenic potential of these cells without CD11b regulation (133).

Mac-1 is also important in establishing peripheral tolerance. CD11b-deficient mice challenged orally with antigen display an overproduction of IL-6 by APCs, leading to high differentiation of T cells into the Th17 subset. These Th17 cells then produce high amounts of IL-17 that interferes with the establishment of oral tolerance and causes exaggerated immune response to inert oral antigen (134). The systemic tolerogenic function of Mac-1 is supported by its effect in lupus mouse models. Fas ${ }^{\text {lpr }}$ mice develop much more severe disease when Mac-1 is deficient. Deletion of structurally similar and familiar integrin LFA-1 had the opposite effect of preventing lupus-like disease (135). Clearly, Mac-1 plays an inhibitory role across many cell types, and its function is tightly regulated and highly specific even when compared to highly similar molecules.

\section{CD11b Regulates B Cell Receptor Signaling}

By convention, CD11b is considered a myeloid cell marker. However, the association of CD11b/ITGAM mutation with SLE, a disease driven by humoral autoimmunity, suggests a function in B cells as well. In 2013, our lab released a study identifying CD11b expression on B cells acting as a negative regulator of BCR signaling. This effectively demonstrated a role in controlling B cell autoimmunity and established the basis for the works of this dissertation (136). 
The known association of ITGAM mutations linked to SLE and the understudied function of $\mathrm{CD} 11 \mathrm{~b}$ in B cells led to this investigation. Initial experiments sought to identify CD11b expression on various B cell populations in anti-snRNP transgenic mice. Here, CD11b was observed in the developmental B cell stages in the bone marrow, follicular, marginal zone, B1a, and B1b B cells. This effectively established the presence of CD11b at all stages of B cells at different expression levels.

In the next phase of the study, the effect of CD11b-deficiency was examined using cells from CD11b global knockout mice. Splenic B cells were stimulated with antiIgM in vitro and it was found that $\mathrm{CD} 11 \mathrm{~b} \mathrm{KO}$ cells hyper-proliferated compared to controls. Interestingly, stimulation with other signals including LPS and CpG did not result in differential proliferation, suggesting this is a BCR-pathway specific phenomenon. Cell viability was enhanced in CD11b deficient cells following IgM stimulation, indicating increased cell survival. Supporting this, cell survival genes were also more highly expressed in KO cells. These cells showed enhanced entry into the $\mathrm{S}$ and G2/M phases of the cell cycle compared to controls. These results outline a role in CD11b for surprising B cell proliferation in response to BCR pathway stimulation. The impact of CD11b global deficiency was then examined in vivo. WT or CD11b KO mice were immunized with apoptotic cells, a major source of auto antigen in SLE. KO mice produced significantly more anti-snRNP antibody and ANAs than controls, as well as a higher frequency of antibody producing PCs. CD11b-deficient mice also had severe Ig deposition in the kidney while controls did not. Because these mice had global CD11b deletion, a chimera model was used to examine the impact of CD11bsufficiency in the B cell developmental stages in the bone marrow. Indeed, these mice 
demonstrated the same autoreactive phenotype following immunization, indicating CD11b impacts autoimmunity after development.

Because CD11b is expressed on many cell types, an adoptive transfer into Rag1defecient mice was used to limit CD11b-knockout to B and T cells. Following apoptotic cell immunization, recipients of CD11b KO cells produced greater auto antibodies and had increased plasma cell frequency compared to controls. The survival of CD11b-KO transferred cells was also greater, correlating with earlier results. B cell deficient $\mu \mathrm{MT}$ mice were utilized in a similar experiment. Again, KO recipients had increased autoimmunity, suggesting a B intrinsic defect.

More in-depth signaling studies were then preformed to examine the mechanism behind CD11b's effect on autoimmunity. Anti-IgM stimulated CD11b KO B cells had decreased levels of Lyn and CD22 phosphorylation. However, many other BCR-vital signaling proteins were unchanged. Calcium influx assay revealed increased calcium flux following BCR crosslinking, as well as increased calcium sensor STIM-1. These results suggest CD11b is involved in the BCR-Lyn-CD22 regulation circuit.

In order to produce a model more relevant to human disease, the next set of experiments used a CD11b-lacking B cell line transfected to express either functional human ITGAM or SNP variant rs1143679. Calcium influx on variant-expressing cells was increased compared to controls, mimicking the knockout phenotype seen in mice. Imagestream analyses were then used to examine the colocalization of major BCR signaling pathway players. Without stimulation, CD11b variant cell lines had significantly decreased colocalization of BCR with CD22 and SHP-1 signals but no change with p-Lyn. After BCR stimulation, variant cells had significantly less BCR 
colocalization with p-Lyn. In both variant and WT expressing cells, CD11b dissociated from the BCR following stimulation. However, variant CD11b significantly decreased colocalization with CD22, p-Lyn, and SHP-1 following stimulation while WT cells did not. These findings demonstrate CD11b mutation impacts the ability of BCR-negative regulators to associate with the BCR.

Clearly, $\mathrm{CD} 11 \mathrm{~b}$ functions as a regulator of $\mathrm{BCR}$ signaling to impact $\mathrm{B}$ cell activation; however, the mechanisms behind this need to be more thoroughly investigated. We hypothesize that B cell intrinsic CD11b is able to regulate the healthy immune response and is not limited to control of autoimmune signaling. Additionally, $\mathrm{CD} 11 \mathrm{~b}$ functions as a BCR signaling regulator in multiple B cell subsets. We will address these unknowns by examining the impact global CD11b deficiency in non-autoimmune animals, specifically deleting CD11b in B cells in vivo, and surveying CD11b expression in various B cell populations under different immune settings. Lastly, we will study the impact of CD11b mutation in human SLE patients to determine its impact on B cell function in disease. 


\section{CHAPTER I}

CD11B REGULATION OF B CELL RECEPTOR SIGNALING IN THE NORMAL IMMUNE RESPONSE 


\section{INTRODUCTION}

Stimulation of a naïve B cell by antigen can result in divergent outcomes. It may become activated and proliferate to generate an immune response, or it can mediate tolerance to the antigen by induction of apoptosis or anergy. The decision between these polar fates is controlled by a number of factors that include the strength of the signal that results from antigen binding of the BCR and the input of co-stimulatory signals that can inhibit or propagate activation. These events also determine the magnitude and longevity of the resulting humoral response. Regulation of these signals is crucial to maintain a healthy level of antibody response that is not excessive and potentially pathogenic or underwhelming and unable to effectively provide immunity against foreign agents.

Our previous work has identified $\mathrm{CD} 11 \mathrm{~b}$ as a negative regulator of BCR signaling in autoimmune animals. The presence of this mechanism implies a function for regulation of the normal immune response as well. However, the autoimmune setting is highly irregular. Alterations to homeostasis and bias of an autoimmune genetic background can skew the normal function of this regulatory factor. In order to better understand the role of $\mathrm{CD} 11 \mathrm{~b}$ as a regulator of $\mathrm{B}$ cell activation, we must examine its effect in a normal, nonautoimmune setting.

\section{BCR Activation Governs B Cell Fate}

The type of stimulation, dose, affinity, and duration of signal can all govern the fate and strength of the B cell response. Effective activation of the BCR is dependent upon a strong, continuous stimulation and the presence of co-stimulatory signals (137). Early stage division following activation is strongly linked to differentiation into class 
switch antibody-producing cells. The magnitude of this critical early division is controlled by the duration and concentration of both T-dependent and innate TLR signals (138). Negative regulations of BCR signaling, which may include CD11b, act as gatekeepers during these vital steps and prevent weakly binding off-target antigens from eliciting a response.

The importance of BCR signaling is not limited to the activation of naïve B cells. Multiple stages of B cell development utilize the BCR to drive proliferation, selection, and survival (139). The GC reaction is vital to the production of an effective high affinity and long-term humoral response and is formed based on BCR signaling. B cells are recruited into the GC or the EF pathway based on affinity for antigen (140). Higher affinity results in more competitive binding to the BCR and subsequent antigen uptake. The increased activation signal from BCR ligation and increased antigen available for $\mathrm{MCH}$ presentation then drive higher duration T-B interactions which govern B cell fate (141). Within the GC, BCR signaling is largely dampened by high activity of SHP-1. This effect is believed to help drive selection via GC B cell dependence on FDC and Tfh help for survival (142). However, a small subset of light zone GC B cells have been found to have active BCR pathway activity (143). These cells may be actively engaging with FDCs to uptake antigen, triggering short-lived BCR pathway activation that is quickly smothered by enhanced negative regulatory signaling. This BCR signaling within GC B cells, along with co-signals like CD40, can drive the expression of vital GC regulators FOXO1 and cMyc (144). FOXO1 regulates GC B selection, and its expression is required for maintaining the dark zone of the GC cycle (145). cMyc is essential for GC maintenance and initiation and limited to the light zone. It is most highly expressed 
following GC B engagement with Tfh cells (146). This demonstrates the critical downstream effects of BCR signaling in driving an effective GC B cell response. Negative Regulation of BCR Signaling is Highly Specific

Ligation of the BCR results in a cascade of downstream signaling events that contribute to activation of the B cell. This signaling pathway is negatively regulated though a system of multi-faceted inhibitors. At the cell surface, Fc $\gamma$ RIIB, CD72, Siglec$\mathrm{G}$, and CD22 co-receptors containing cytoplasmic immunoreceptor tyrosine-based inhibition motifs (ITIMs) become phosphorylated via Lyn following BCR ligation (147). These phosphorylated regions are then able to recruit phosphatases, such as SHP-1, which can inactive phosphorylation signals downstream of the BCR including $\mathrm{PIP}_{3}$, and Syk $(148,149)$. These various surface co-receptors negatively regulate through different downstream effectors. CD22 acts mainly through SHP-1, while Fc $\gamma$ RIIB can only recruit SHIP-1 (147). Indeed, each of these inhibitory co-receptors has been demonstrated to serve distinct regulatory functions. CD72 recognizes self-antigen Sm/RNP, and acts as an autoimmune regulator (150). CD22 regulates the constantly low level activation of BCR known as tonic signaling, which serves to promote resting B cell survival (151). Similarly, Siglec-G maintains B-1 B cell homeostasis (152). Our previous study found that CD11b deficiency caused more severe disease in Sn/RNP autoimmune mice, possibly via defects in CD72. However, CD11b was also necessary to maintain association of CD22 and SHP-1 with the BCR upon stimulation, suggesting a role in controlling tonic signaling. These findings suggest a larger role for CD11b in controlling BCR activation, influencing different regulatory pathways that are not limited to autoreactive tolerance. 
In the following experiments, we examine the effect of CD11b deficiency in a Th2 immunization setting to determine its function on B cells in the normal immune response. We found that a global loss of CD11b leads to an increased antibody response, as well as higher amounts of antigen-specific GC and PCs. In vitro examination of GC BCR signaling reveals defects in the SHP-1 and pLyn negative regulation pathway and altered utilization of critical GC regulatory factors FOXO1 and cMyc. These data describe a novel role for CD11b in regulating a healthy immune response, as well as suggest a function in controlling BCR signaling at multiple stages of B cell development. 


\section{MATERIALS AND METHODS}

Mice

NP Tg CD11b ${ }^{-/-}$knockout mice (NP KO) were generated by interbreeding CD11b global knockout mice with NP Tg mice (The Jackson Laboratory). CD11b ${ }^{\text {loxp/loxp }}$ mice were generated for the purpose of this study using CRISPR/Cas9. Conditional knockout mice

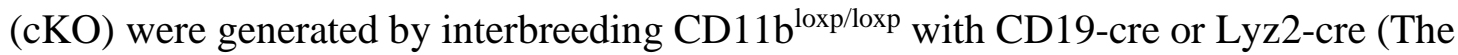
Jackson Laboratory) mice to obtain CD19;cre-CD11b loxp/loxp $_{\text {and Lyz2-cre;CD11b }}^{\text {loxp/loxp }}$ mice, respectively. Mice at 6-8 weeks of age with both sexes were used for immunization experiments. All mice were housed and bred in a conventional facility at the University of Louisville. Animal care and experiments were conducted in accordance with the National Institutes of Health guidelines and were approved by the Institutional Animal Care and Use Committee at the University of Louisville.

\section{Immunizations and Ab Detection}

$\mathrm{NP}$ tg or NP KO mice were immunized via i.p. injection of $50 \mu \mathrm{g} \mathrm{NP}_{20-29}-\mathrm{CGG}$ in Alum adjuvant at a 1:1 ratio. Conditional knockout or control mice were immunized via intraperitoneal (i.p.) injection of $100 \mu \mathrm{g}$ OVA peptide or 50 $\mu \mathrm{g} \mathrm{NP}_{20-29}$-CGG (Biosearch Technologies) in Alum adjuvant at a 1:1 ratio. Anti-NP and anti-OVA and antibody titers were measured via enzyme-linked immunosorbent assay (ELISA) using a 96-well plate coated with $1 \mu \mathrm{g}$ of OVA or $\mathrm{NP}_{22}-\mathrm{BSA}$ and a standard ELISAMAX kit (Biolegend).

Antibodies, Flow Cytometry, and Cell Sorting 
Single-cell suspensions were obtained from spleen and treated with Fc-blocker for 10 minutes on ice followed by staining with appropriate fluorochrome-labeled antibody or isotype controls for at least 20 minutes at $4^{\circ} \mathrm{C}$. Cells were then washed with 10 volumes of PBS before resuspension in PBS for acquisition or Running Buffer (Miltenyi Biotech) for sorting. For intracellular staining, cells were fixed and permeabilized in appropriate buffers (Biolegend) followed by the addition of fluorochrome-labeled antibody. Cells were then washed two times with 10 volumes of permeabilization buffer and resuspension for acquisition. Flow cytometry samples were acquired using a FACSCanto cytometer (BD Biosciences) and analyzed using FlowJo software (Tree Star). Sorting was performed using FACSAria III (BD Biosciences) or MoFlo XDP (Beckman Coulter). Cell purity was confirmed at greater than $90 \%$ via flow cytometry. The following antimouse antibodies were used: IgD, GL7, B220 (CD45R), CD21/35, CD23, CD38, CD80, CD138 (Biolegend).

\section{Quantitative real-time PCR}

Sorted GC B cells and plasma cells were suspended in TRIzol (Life Technologies) for lysis and storage, followed by RNA extraction described in the manufacturer protocol. RNA was used to transcribe cDNA using a Reverse Transcription Kit (Bio-Rad). Quantitative PCR was then performed on a Bio-rad MyiQ single-color RT-PCR detection system using SYBR Green Supermix (Bio-Rad). Primer sequences used: Blimp1 F'5AGAGTGCACAGTGGAGAACG-3'; Blimp1 R'5-GGTGCACAAATTGCGTAAAC-3'; Bcl-XL F'5-TCCCGTAGAGATCCACAAAAGT-3’; Bcl-XL: R'5GACAAGGAGATGCAGGTATTGG-3'. Gene expression was normalized to the $\beta_{2-}$ 
microblobulin ( $\beta-M G)$ housekeeping gene and represented data as the fold differences by the $2^{-\Delta \Delta \mathrm{Ct}}$ method, where the threshold cycle $(\Delta \mathrm{Ct})=\mathrm{Ct}_{\text {arget gene }}-\mathrm{Ct}_{\beta-\mathrm{MG}}$ and $\Delta \Delta \mathrm{Ct}=$ $\Delta \mathrm{Ct}_{\text {induced }}-\Delta \mathrm{Ct}_{\text {reference }}$

\section{Imagestream Analysis}

Whole spleens were resuspended at $20 \times 10^{6}$ cells $/ \mathrm{mL}$ in RPMI-1640 medium (Sigma, St. Louis, MO) (supplemented with 10\% FBS, 5.5 $\times 10^{-5} \mathrm{M}$ 2-ME, 100 units $/ \mathrm{ml}^{-1}$ penicillin, and $100 \mu \mathrm{g} / \mathrm{mL}^{-1}$ streptomycin (GIBCO, Waltham, MA)) and pre-warmed at $37^{\circ} \mathrm{C}$ for 30 minutes. Cells were stimulated with $20 \mu \mathrm{g} / \mathrm{mL}$ FITC-anti-IgM F(ab' $)_{2}$ for indicated time points. Stimulation was immediately stopped by addition of PFA to $1.5 \%$. Cells were washed with PBS and treated with Fc Blocker before antibody staining for surface molecules. Cells were then washed with PBS again followed by permeabilization with 0.1\% Triton-X 100 in Staining Buffer (PBS with 3\% FBS, 0.02\% Azide, and 2mM EDTA). Cells were then washed with Staining Buffer and incubated overnight at $4^{\circ} \mathrm{C}$ with antibodies for intracellular molecules. Cells were washed in Staining Buffer and incubated with secondary antibody if necessary, then resuspended in $100 \mu \mathrm{L}$. Samples were acquired using the Amnis Imagestream-X (EMD Millipore) and analyzed with IDEAS software (EMD Millipore). Colocalization was calculated using the similarity feature.

\section{Statistical Analysis}

All quantitative data are shown as mean \pm s.e.m unless otherwise indicated. All samples were compared using two-tailed, unpaired Student's $T$ test. A $P$ value less than 0.05 was 
considered significant. Statistical analysis was performed with Prism (Graphpad Software). 
RESULTS

Global CD11b deficiency alters antibody production and GC formation in nonautoimmune mice.

To examine the effects of CD11b deficiency in an antigen-specific and nonautoimmune Th2 setting, we crossed $\mathrm{CD} 11^{-/-}$mice with the transgenic NP mouse model. These animals carry a pre-recombined BCR heavy chain variable locus that has affinity for the hapten NP (4-hydroxy-3-nitrophenylacetyl). The advantages of this system are twofold. Firstly, it allows NP antigen-specific cells to be identified via antibody staining. Secondly, these mice carry a higher frequency of innate anti-NP B cells, allowing for a greater scale response following immunization. $\mathrm{NPtg}_{\mathrm{CD}} 11 \mathrm{~b}^{-/-}$mice and $\mathrm{NPtg} \mathrm{CD} 11 \mathrm{~b}^{+/+}$ controls were immunized with $50 \mu \mathrm{g}$ of NP-CGG (NP-chicken gamma globulin conjugate) suspended in Alum adjuvant. In mice, Alum causes a heavily polarized Th2type response that enhances antibody production (153). Alum has also been suggested as a preferential adjuvant for use over Complete Freund's Adjuvant (CFA), which can cause harmful and interfering off-target effects (154).

Animals were sacrificed at day 14 post-immunization to allow for full formation of the GC reaction and production of antibody-producing PCs. Anti-NP IgG effectorclass antibody was elevated in CD11b-knockout mice compared to controls. Baseline IgM class antibody was also increased in knockout mice, which is produced by non-class switched, non-GC B cells (Figure 1). These heightened antibody levels indicate a dysregulation of the $\mathrm{B}$ cell response to immunization in the absence of CD11b, revealing a role not limited to autoimmune reactions. 
The notable increases in serum antibody then led us to investigate the status of GC and PC populations in this model. At 14 days after immunization, we did not observe any significant change in the total amount of GC B cells, averaging at $0.65 \%$ of splenic lymphocytes. However, when examining the NP-specific cell population, we did find an increased frequency of GCs, and both subsets were notably higher than their non-specific counterparts at roughly $1 \%$ and $2 \%$ for control and CD11b deficient animals, respectively (Figure 2). Examination of the PC population revealed higher frequency in knockout mice. Unlike the GCs, total PCs were increased in addition to the NP-specific subset (Figure 3). Increases in both of these populations suggest CD11b-negative regulation is able to limit the high affinity antibody response, though the antigen-restriction of this effect in GCs but not PCs implies a more dynamic effect between these stages of B cell maturation.

To further profile these cells, we sorted GC B cells and PCs from immunized mice to assess expression of Bcl-XL, an anti-apoptotic and B-cell differentiation regulator, and Blimp1, which is vital for PC differentiation. For GCs, both Blimp1 and Bcl-XL were more highly expressed in knockout cells. PCs significantly increased Blimp1 expression, but not Bcl-XL (Figure 4). Together, these data demonstrate an antigen-restricted negative-regulatory role for $\mathrm{CD} 11 \mathrm{~b}$ on the maintenance of GC B and PC populations, which impacts the magnitude of the antibody response. 

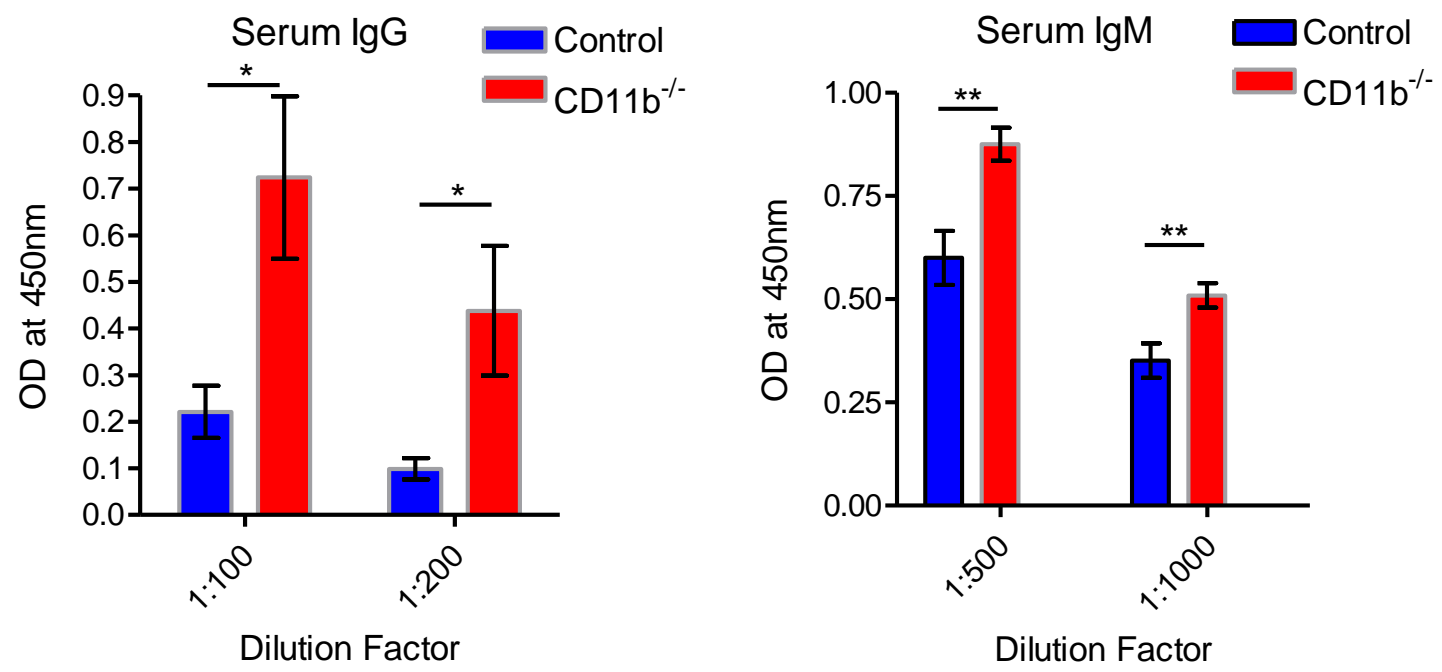

Figure 1: Increased antibody response in CD11b-knockout mice following immunization. $\mathrm{NPtg} \mathrm{CD}_{11} \mathrm{~b}^{-/}$and $\mathrm{NPtg} \mathrm{CD} 11 \mathrm{~b}^{+/+}$mice were immunized with $50 \mu \mathrm{g}$ of NP-CGG suspended in Alum via i.p. injection. Serum was collected on day 14 postimmunization. Anti-NP IgG and IgM antibody was measured via ELISA in plates coated with $1 \mu \mathrm{g} /$ well of NP-BSA. Optical density was measured at 450nm. Data are means \pm s.e.m. Summarized data are from at least three independent experiments. ${ }^{*} \mathrm{P}<0.05 ; * * \mathrm{P}<0.01$ (unpaired two-tailed Student's t-test). 

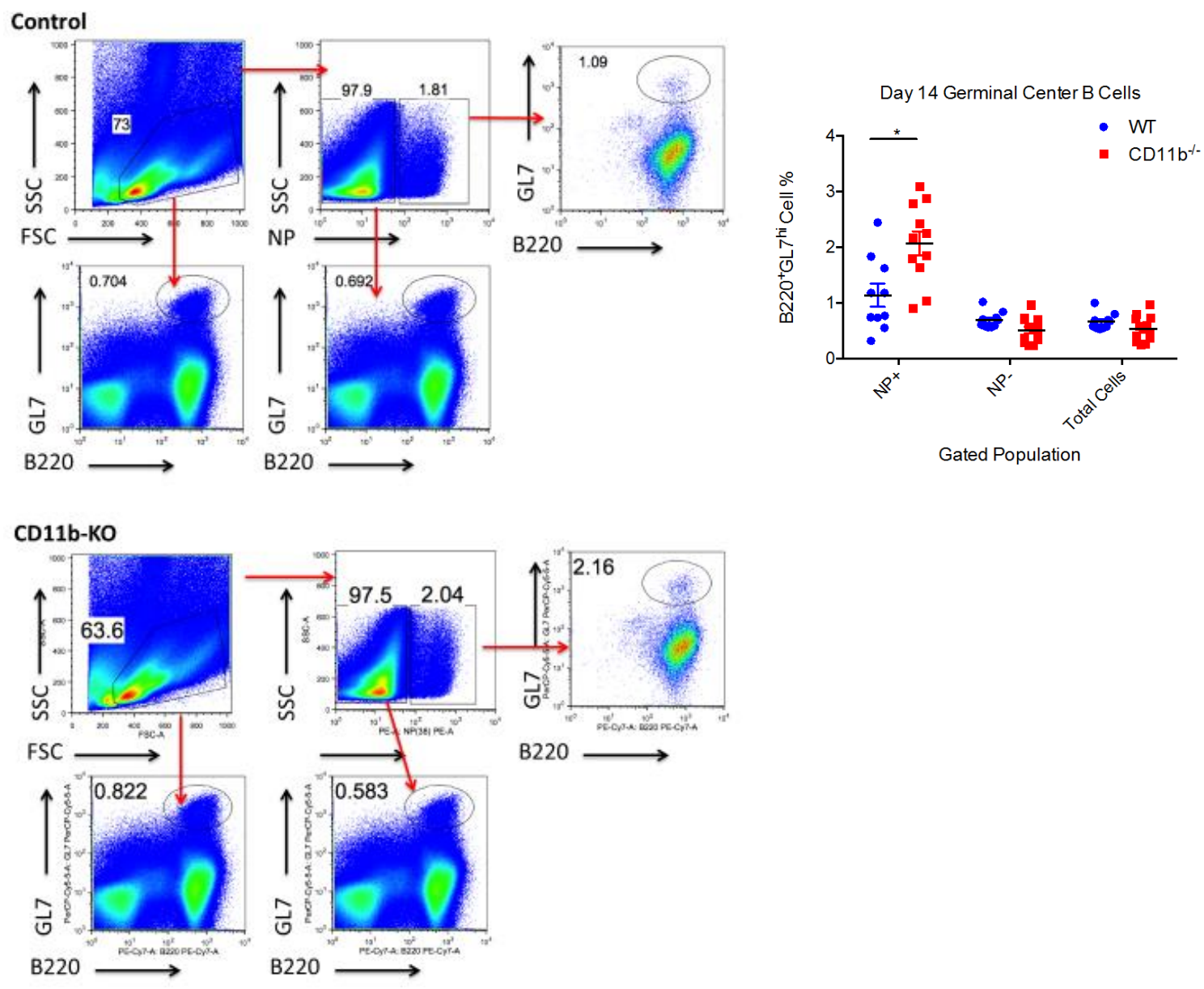

Figure 2: Germinal center B cells are increased in CD11b-knockout mice. NPtg $\mathrm{CD}_{11} \mathrm{~b}^{-/-}$and NPtg CD11 $\mathrm{b}^{+/+}$mice were immunized with $50 \mu \mathrm{g}$ of NP-CGG suspended in Alum via i.p. injection. Spleens were harvested on day 14 post-immunization. Gating strategy and representative dot-plots of control (top) and knockout (bottom) mice. Summarized data for GC frequency is shown on right. Data are means \pm s.e.m. Summarized data are from at least three independent experiments. $* \mathrm{P}<0.05$ (unpaired two-tailed Student's t-test). 


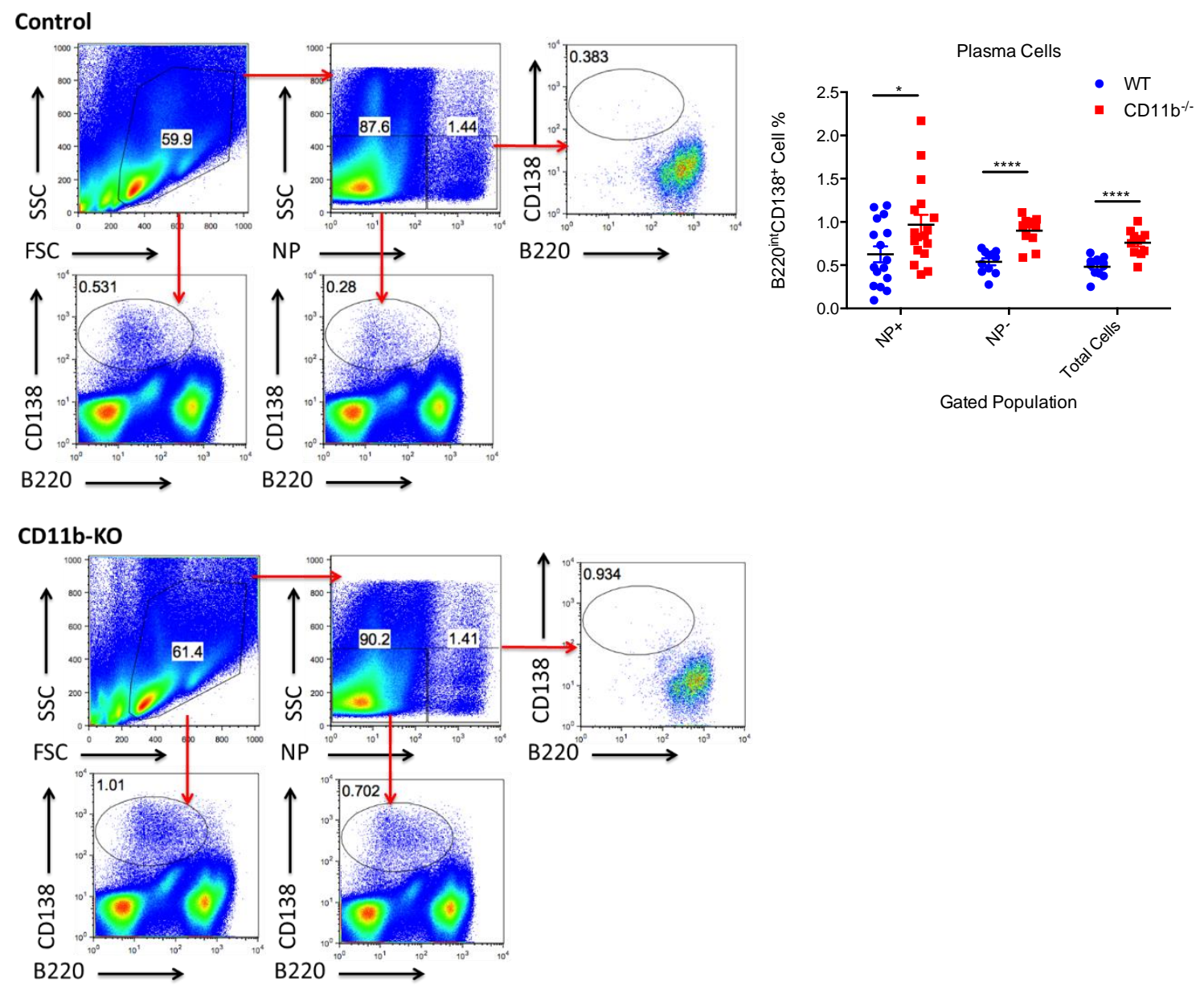

Figure 3: Plasma cells are increased in CD11b-knockout mice. NPtg CD11 $\mathrm{b}^{-/}$and NPtg CD11 $\mathrm{b}^{+/+}$mice were immunized with $50 \mu \mathrm{g}$ of NP-CGG suspended in Alum via i.p. injection. Spleens were harvested on day 14 post-immunization. Gating strategy and representative dot-plots of control (top) and knockout (bottom) mice. Summarized data for GC frequency is shown on right. Data are means \pm s.e.m. Summarized data are from at least three independent experiments. ${ }^{*} \mathrm{P}<0.05$ (unpaired two-tailed Student's t-test). 

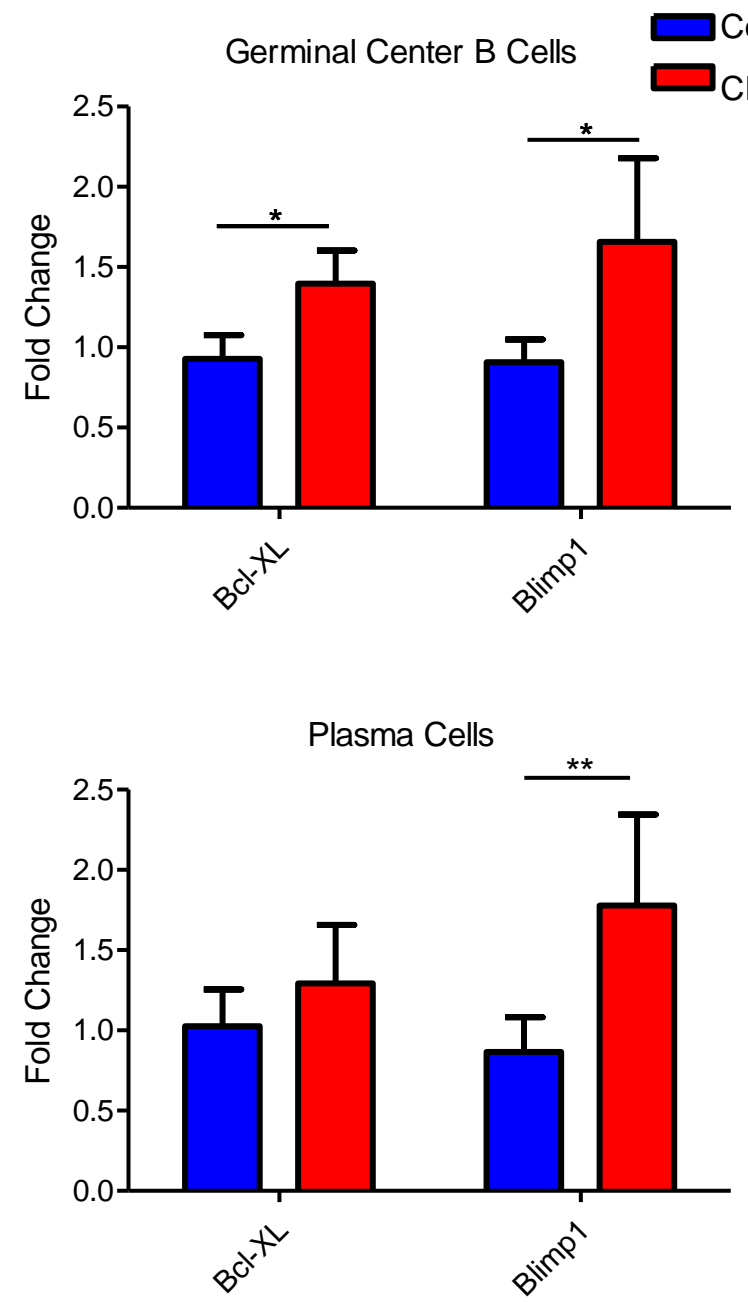

Figure 4: CD11b deficiency alters GC and PC regulator expression. GCBs and PCs were sorted from immunized NPtg CD11 $\mathrm{b}^{-/}$and $\mathrm{NPtg} \mathrm{CD} 11 \mathrm{~b}^{+/+}$mice on day 14 . RNA was extracted, used to transcribe cDNA, and RT-PCR was performed to examine gene expression of genes Bcl-XL and Blimp1. Data are means \pm s.e.m. Summarized data are from at least two independent experiments. $* \mathrm{P}<0.05 ; * * \mathrm{P}<0.001$ (unpaired two-tailed Student's t-test). 


\section{CD11b Regulates Germinal Center B Cell BCR Signaling}

Our previous study demonstrated the role of $\mathrm{CD} 11 \mathrm{~b}$ as a negative regulator of BCR signaling in vitro using the K46-17 B cell line transfected with a mutant form of human ITGAM. Here, we utilize the NP CD11b global knockout mouse model to examine BCR signaling on GC B cells ex vivo using Imagestream. This imaging flow cytometry provides microscopy visualization of cells based on flow cytometry gating, allowing for the acquisition of large numbers of events and rare cell populations that would be unobtainable using traditional confocal microscopy methods. These visual data can then be analyzed using associated software and provide quantitative numerical results to accompany qualitative visual images. Control and CD11b deficient mice were immunized in the same manner as previous experiments and splenocytes were analyzed at 14 days post treatment. Single cell suspensions of spleen were stimulated with FITClabeled anti-IgM Fab fragments lacking the Fc portion of the molecule, allowing for divalent binding of the $\mathrm{BCR}$ without nonspecific binding of $\mathrm{Fc}$ receptors found on $\mathrm{B}$ cells.

We first examined the impact of CD11b deficiency on BCR signaling molecules SHP-1, pLyn, and pSyk. As previously discussed, SHP-1 and pLyn negatively regulate the BCR signaling cascade by de-phosphorylating activation signals such as pSyk. Functional association of the BCR with each respective molecule was determined by measuring overlap of fluorescent signal across the cell, quantified as a colocalization score. BCR stimulation induced colocalization with negative regulator SHP-1 in both groups, but this was significantly reduced in CD11b knockout cells (Figure 5). In wildtype cells, pLyn was colocalized with the BCR in the resting state, briefly separated after 
5 minutes of stimulation, then rejoined by the 10-minute time point. CD11b deficient cells maintained a very low level of pLyn/BCR localization that was not altered by BCR ligation (Figure 6). When investigating pSyk, we did not see a significant change in colocalization with the BCR in CD11b deficient GC B cells versus controls (Figure 7). However, non-GC B cells did display a significant decrease in the absence of CD11b, suggesting differential regulation of this activator between B cell subsets (Figure 8). The decreased association of negative regulatory elements SHP-1 and pLyn with the BCR in knockouts show that $\mathrm{CD} 11 \mathrm{~b}$ serves as a signal regulator in the GC, consistent in its role shown with our previous data at the naïve B cell stage.

Next we investigated the nuclear localization activity of cMyc and FOXO1, which are involved in GC survival and selection (144). A significant reduction in nuclear localization of cMyc was observed in CD11b deficient GC B cells both before and after stimulation (Figure 9). BCR stimulation caused an increase in cMyc nuclear activity in wild-type cells, but knockout-cell cMyc activity was less affected. FOXO1 nuclear translocation was also unchanged by anti-IgM stimulation in both groups, though there was a consistent significant reduction in knockout mice (Figure 10). The fate of GC B cells is driven by expression of these key regulators, which are activated by BCR and coreceptor signaling. Altered nuclear utilization of FOXO1 and cMyc in the absence of CD11b reveals the downstream functional impact of its role in BCR negative regulation. The significant changes in these general B cell and GC B-specific factors outline a mechanism for $\mathrm{CD} 11 \mathrm{~b}$ regulation of $\mathrm{BCR}$ signaling in vivo at the $\mathrm{GC}$ stage. 

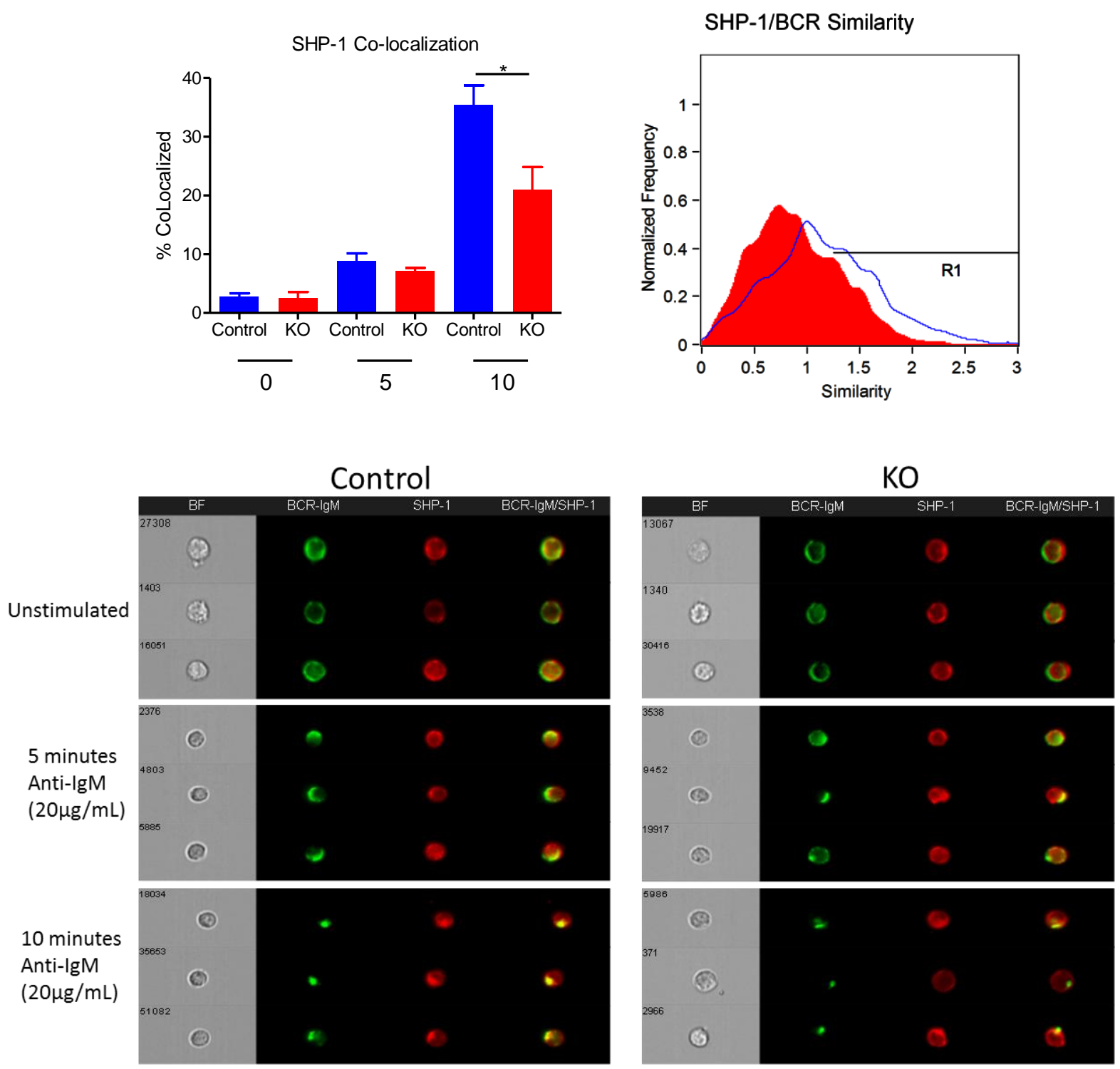

Figure 5: Loss of CD11b alters SHP-1 colocalization with the BCR. Single-cell

suspensions of day 14 post-immunization spleens from NPtg CD11b ${ }^{-/-}$and NPtg

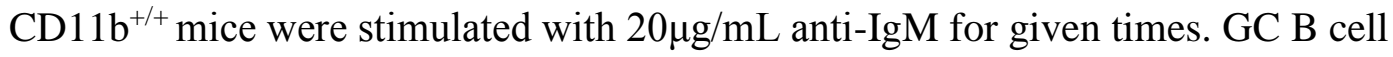
colocalization of SHP-1 and BCR was measured via Imagestream, gated on B220 2 GL $7^{\text {hi }}$ events and defined by a similarity score greater than 1.25 . Summary data for percentage of nuclear localized cells, representative similarity histogram, and representative images are shown. Data are means \pm s.e.m. $* \mathrm{P}<0.05$ (unpaired two-tailed Student’s t-test). 

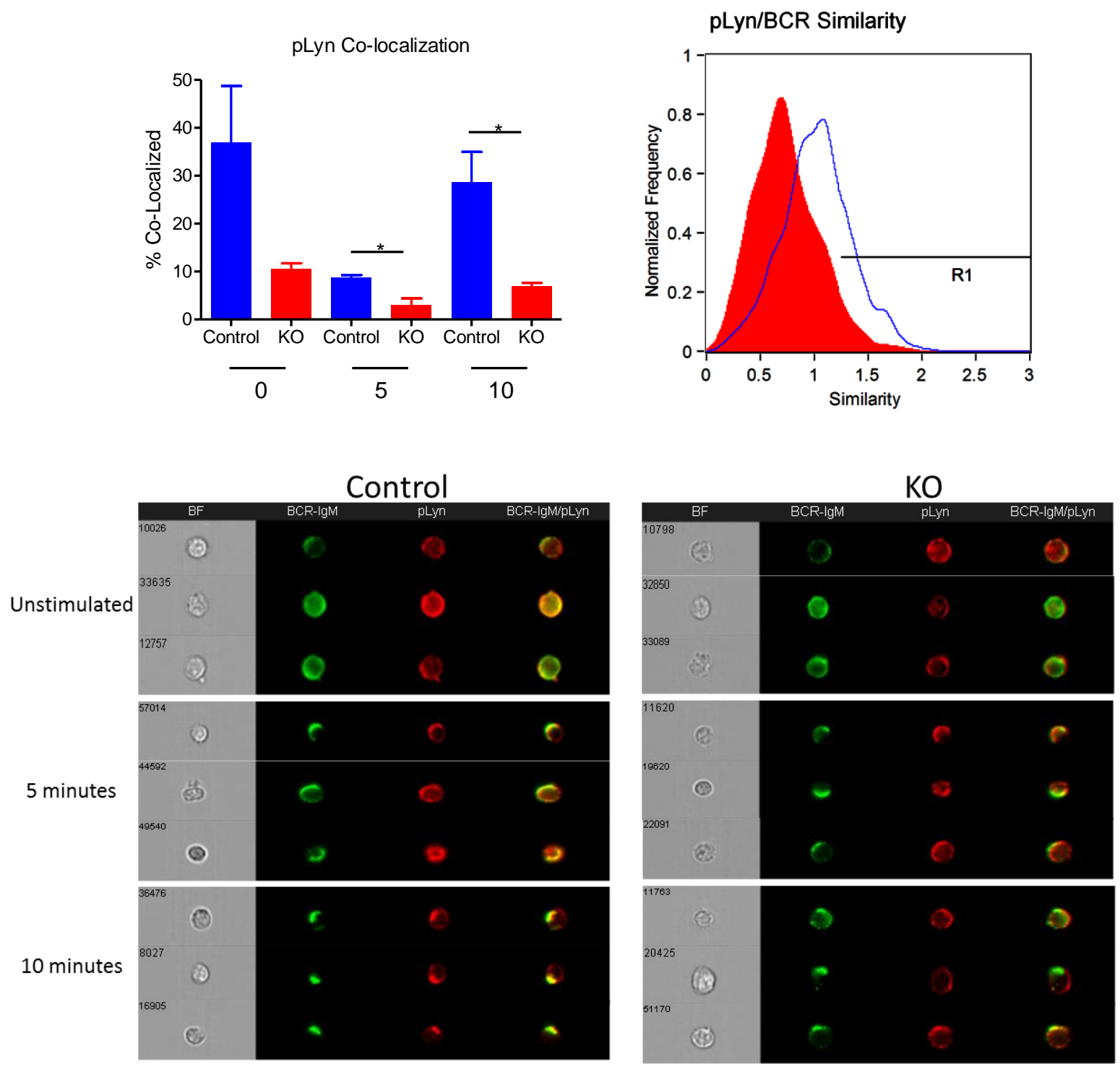

Figure 6: Loss of CD11b alters pLyn colocalization with the BCR. Single-cell

suspensions of day 14 post-immunization spleens from NPtg CD11 ${ }^{-/-}$and $\mathrm{NPtg}^{-}$

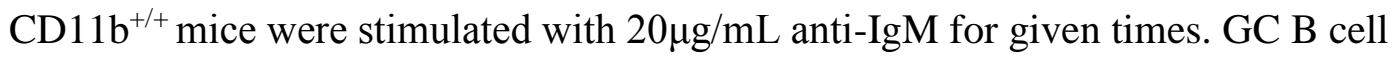
colocalization of pLyn and BCR was measured via Imagestream, gated on B $220^{+} \mathrm{GL} 7^{\text {hi }}$ events and defined by a similarity score greater than 1.25 . Summary data for percentage of nuclear localized cells, representative similarity histogram, and representative images are shown. Data are means \pm s.e.m. $* \mathrm{P}<0.05$ (unpaired two-tailed Student's t-test). 

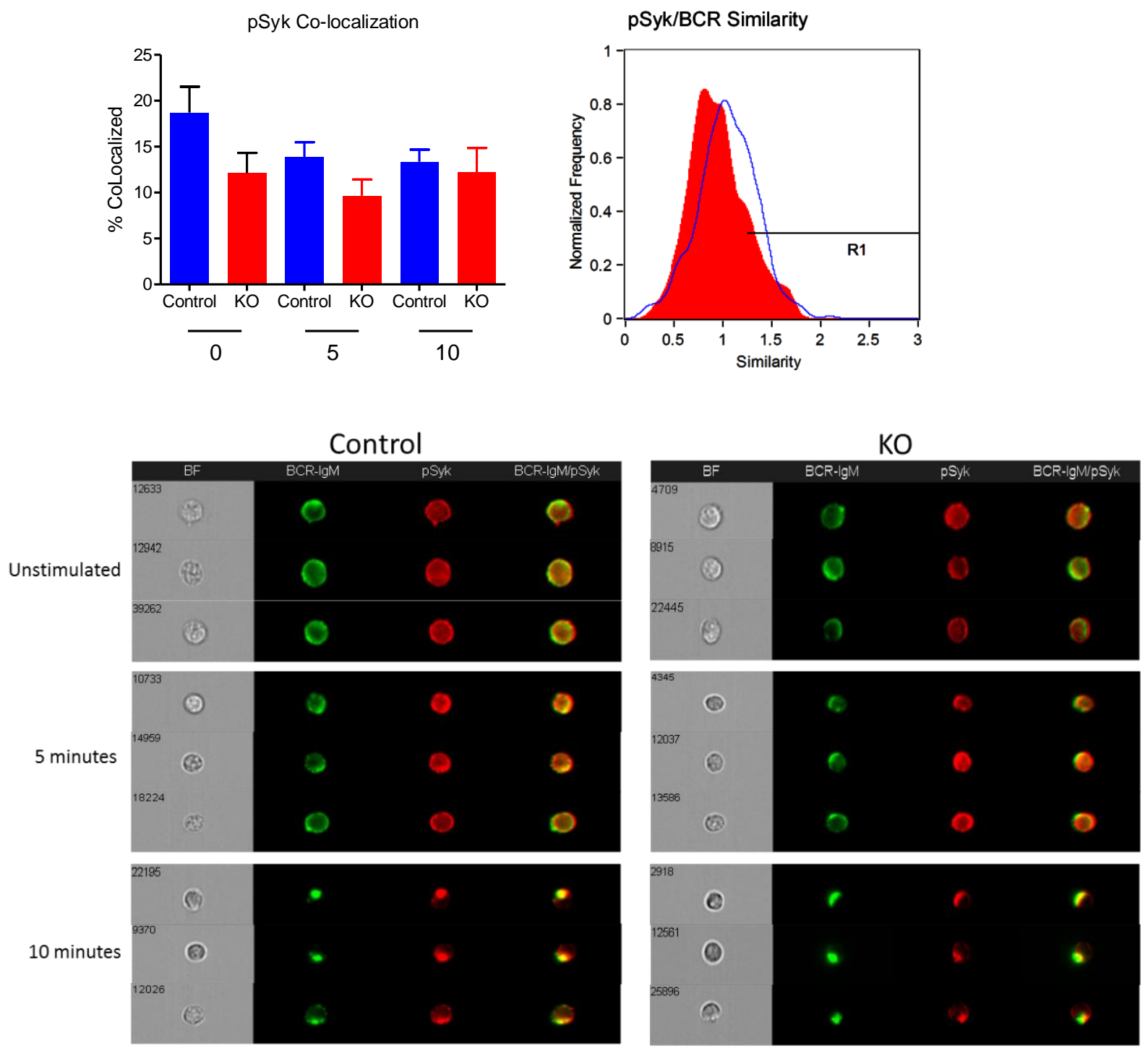

Figure 7: Loss of CD11b and pSyk colocalization with the BCR. Single-cell

suspensions of day 14 post-immunization spleens from NPtg CD11b ${ }^{-/-}$and NPtg CD $11 b^{+/+}$mice were stimulated with $20 \mu \mathrm{g} / \mathrm{mL}$ anti-IgM for given times. GC B cell colocalization of pSyk and BCR was measured via Imagestream, gated on B220+GL $7^{\text {hi }}$ events and defined by a similarity score greater than 1.25 . Summary data for percentage of nuclear localized cells, representative similarity histogram, and representative images are shown. Data are means \pm s.e.m. $* \mathrm{P}<0.05$ (unpaired two-tailed Student's t-test). 

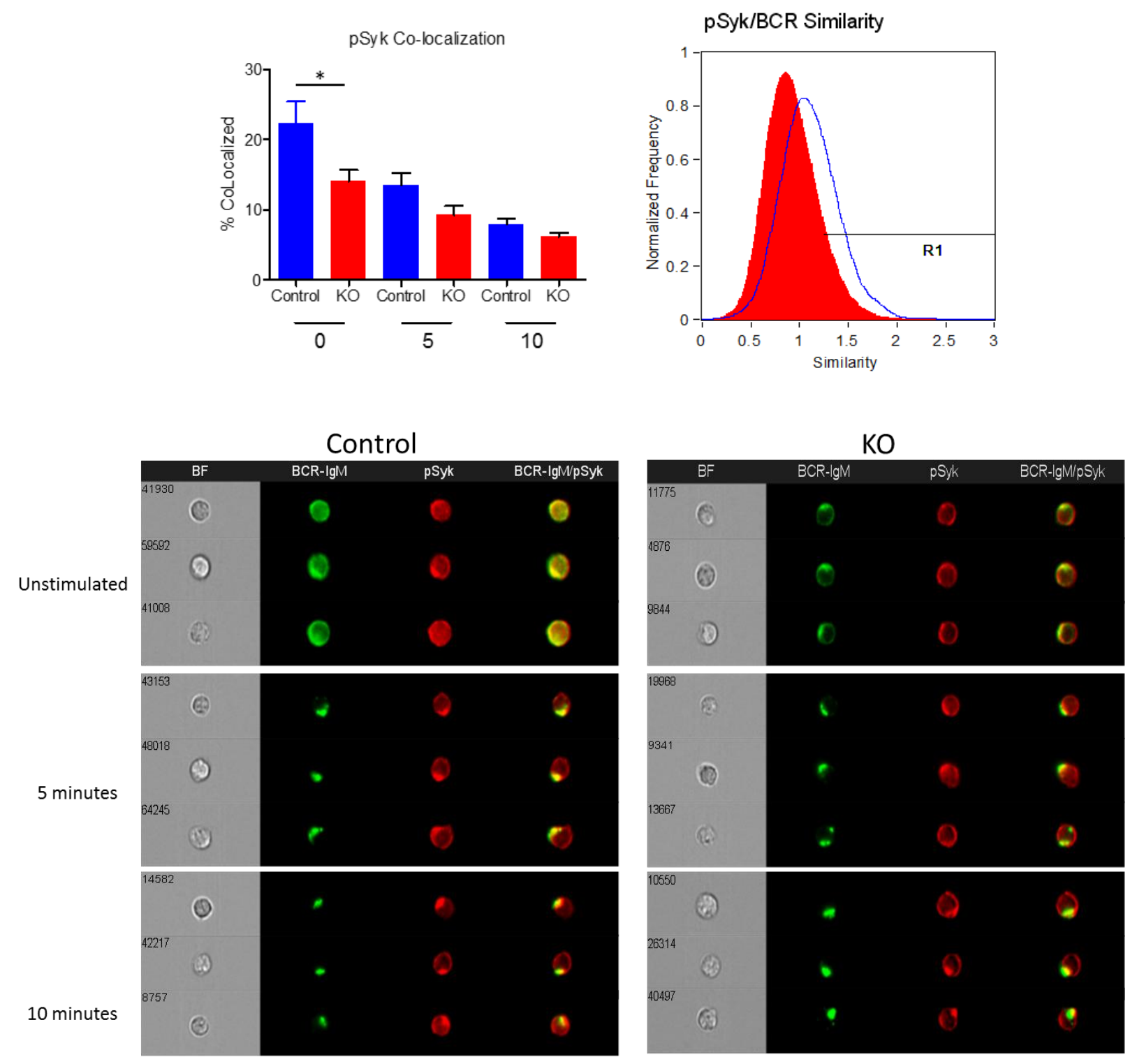

Figure 8: CD11b deficiency alters pSyk colocalization in non-GC B cells . Single-cell

suspensions of day 14 post-immunization spleens from NPtg CD11b ${ }^{-/-}$and NPtg $\mathrm{CD}_{11} \mathrm{~b}^{+/+}$mice were stimulated with $20 \mu \mathrm{g} / \mathrm{mL}$ anti-IgM for given times. Non-GC B cell colocalization of pSyk and BCR was measured via Imagestream, gated on B220 ${ }^{+} \mathrm{GL7}^{-}$ events and defined by a similarity score greater than 1.25 . Summary data for percentage of nuclear localized cells, representative similarity histogram, and representative images are shown. Data are means \pm s.e.m. $* \mathrm{P}<0.05$ (unpaired two-tailed Student’s t-test). 

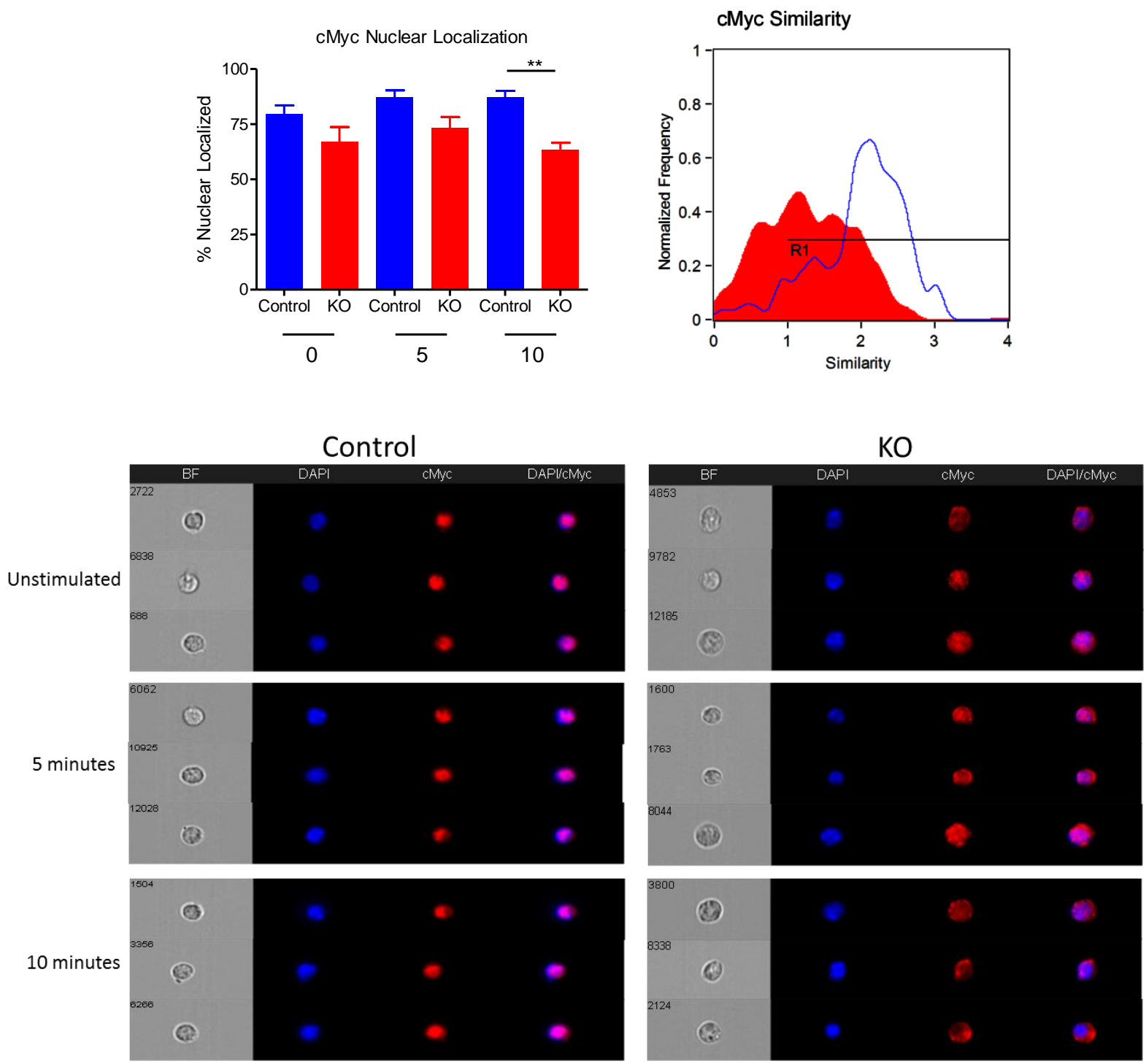

Figure 9: Loss of CD11b alters cMyc Nuclear Translocation. Single-cell suspensions of day 14 post-immunization spleens from $\mathrm{NPtg}^{\mathrm{CD}} 11 \mathrm{~b}^{-/-}$and $\mathrm{NPtg} \mathrm{CD} 11 \mathrm{~b}^{+/+}$mice were stimulated with $20 \mu \mathrm{g} / \mathrm{mL}$ anti-IgM for given times. GC B cell nuclear localization of cMyc was measured via Imagestream, gated on B220+GL $7^{\text {hi }}$ events and defined by a similarity score greater than 1 . Summary data for percentage of nuclear localized cells, representative similarity histogram, and representative images are shown. Data are means \pm s.e.m. $* * \mathrm{P}<0.001$ (unpaired two-tailed Student's t-test). 

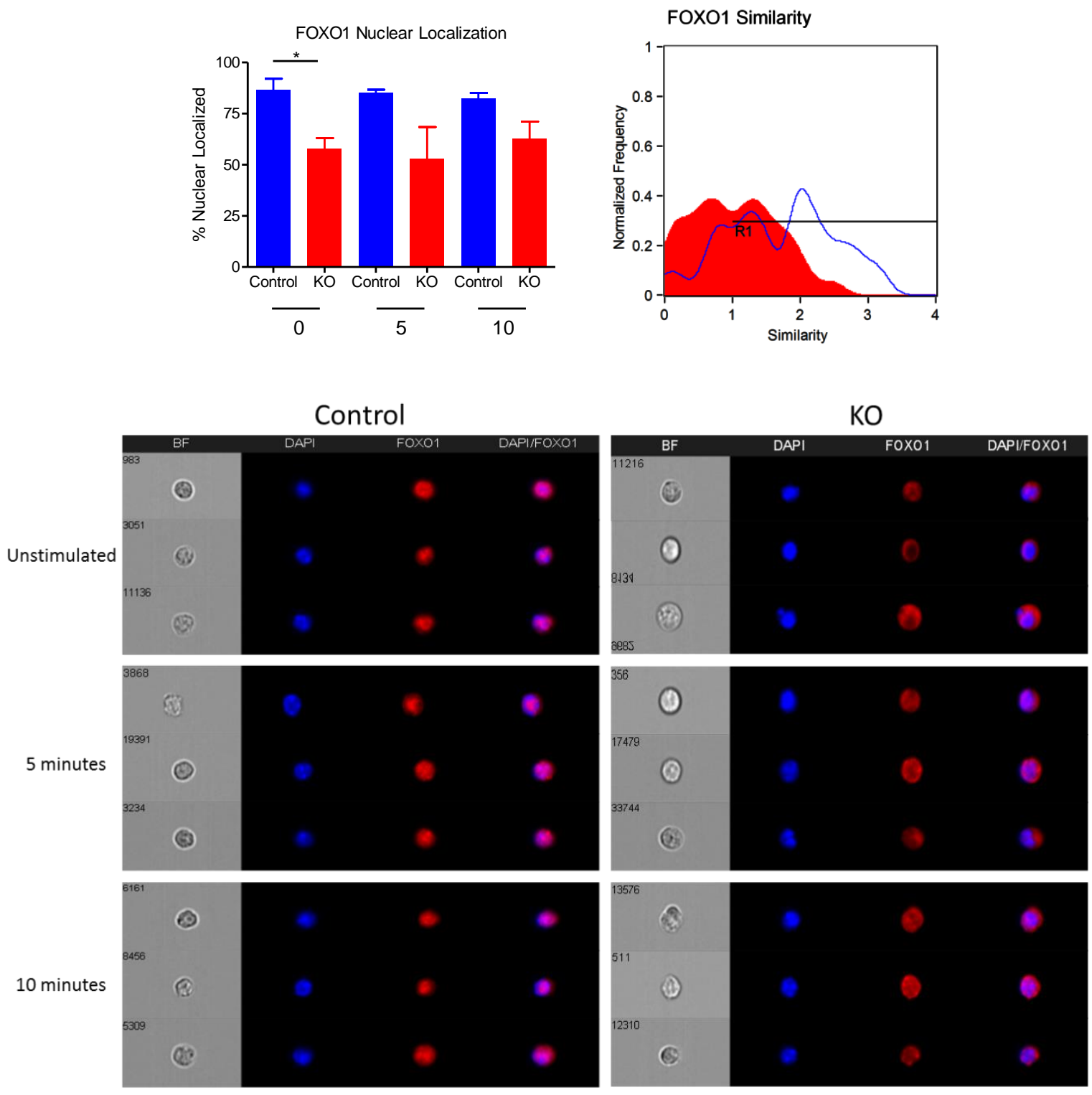

Figure 10: Loss of CD11b alters FOXO1 Nuclear Translocation. Single-cell suspensions of day 14 post-immunization spleens from NPtg CD11b ${ }^{-/-}$and NPtg

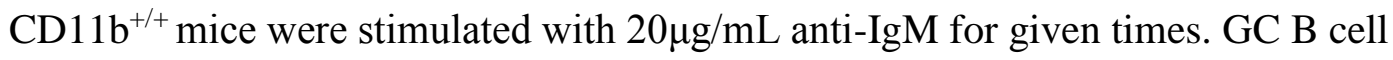
nuclear localization of FOXO1 was measured via Imagestream, gated on B220 $0^{+} 7^{\text {hi }}$ events and defined by a similarity score greater than 1 . Summary data for percentage of nuclear localized cells, representative similarity histogram, and representative images are shown. Data are means \pm s.e.m. ${ }^{*} \mathrm{P}<0.05$ (unpaired two-tailed Student's t-test). 


\section{$B$ cell intrinsic $\mathrm{CD11b}$ regulates the $\mathrm{Th} 2$ antibody response}

The expression of CD11b on B cells and its impact on BCR signaling has been clearly demonstrated by our previous experiments. However, these studies have either taken place in vitro conditions or with in vivo global knockout models. The increased B cell response seen in our NP experiments could be caused by CD11b deficiency in several different cell types vital to the Th2 response and GC formation including Tfh cells, macrophages, and FDCs. To better elucidate the cell-specific importance of CD11b in this phenotype, we generated an inducible knockout model using the cre-lox system (Figure 11). We first crossed this strain with CD19-cre mice to study the effect of a B cell-restricted CD11b deletion.

CD11b ${ }^{\text {loxp/loxp }}$ and CD11b $b^{\text {loxp/loxp }} ;$ CD19-Cre littermates were immunized with OVA to induce a Th2 type reaction, analogous to our previous experiments. At 14 days post-immunization there was a significant increase in anti-OVA IgG antibody while an increase in antigen-specific IgM antibody was also observed, mimicking the phenotype of global CD11b knockouts (Figure 12). Examination of the GC frequency reveals a possible trending but not statistically significant increase in knockout mice. Surprisingly, the PC population was completely unaltered despite the observed increase in antibody response (Figure 13). Because of the high CD11b expression levels found on follicular, marginal zone, and memory B cells in our reporter studies, we investigated these populations as well; however, no difference in the frequencies of any of these B cell types was noted (Figure 14). These results suggest that B cell CD11b expression is regulating the antibody response intrinsically, but effects on the frequency of B cell populations are subtler. 

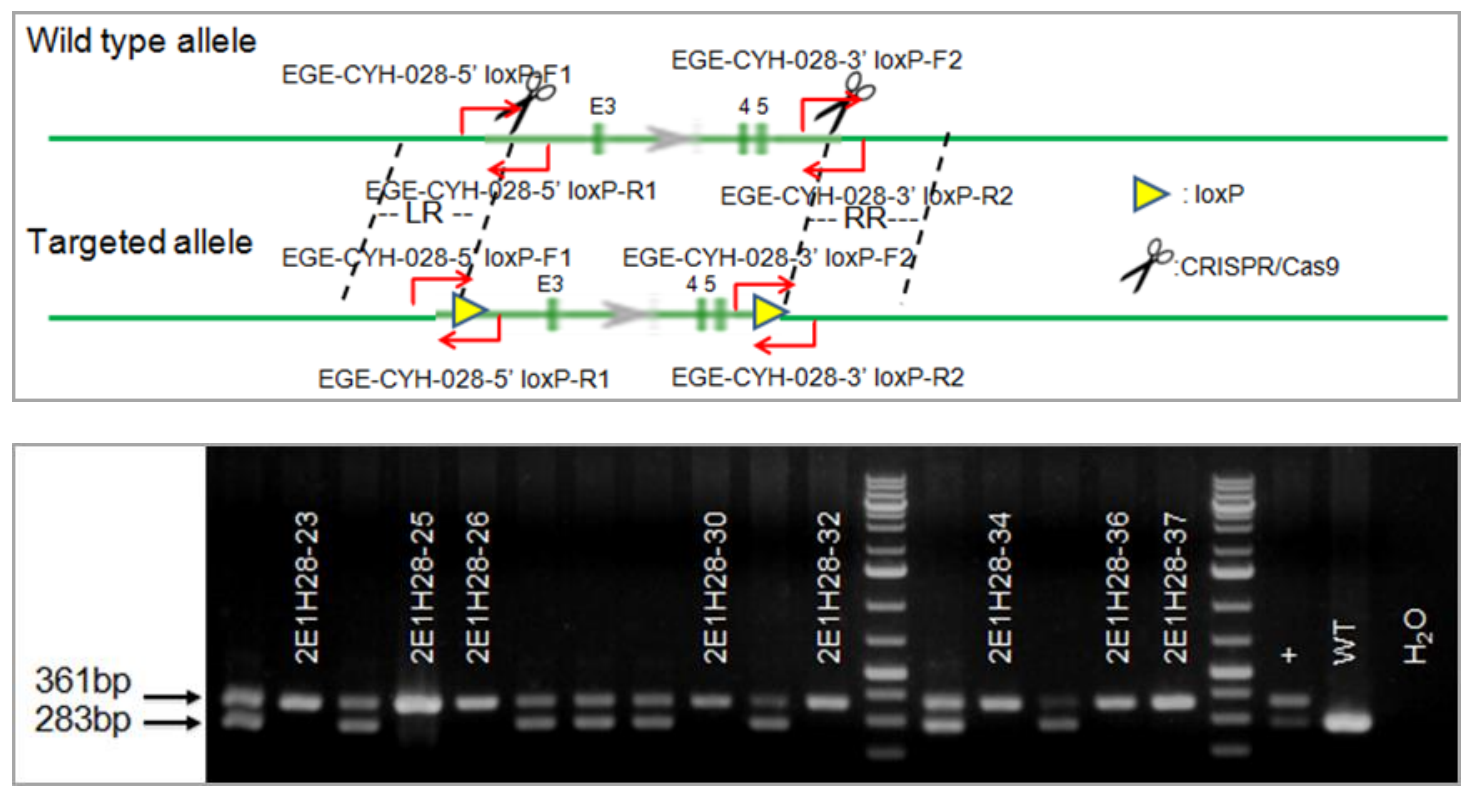

Figure 11: Generation of CD11b-loxp mice. CD11b conditional knockout mice were generated for this purpose of this study using CRISPR/Cas9. Construct design (top).

Briefly, loxP elements were inserted into intron 2 and intron 5, resulting in loxP flank of exons $3-5$ and causing a frame shift resulting in a truncated non-sense mediated decay protein. Example genotyping results are shown (bottom). The wild-type allele results in a $283 \mathrm{bp}$ band, while the loxp mutant can be seen at $361 \mathrm{bp}$. 

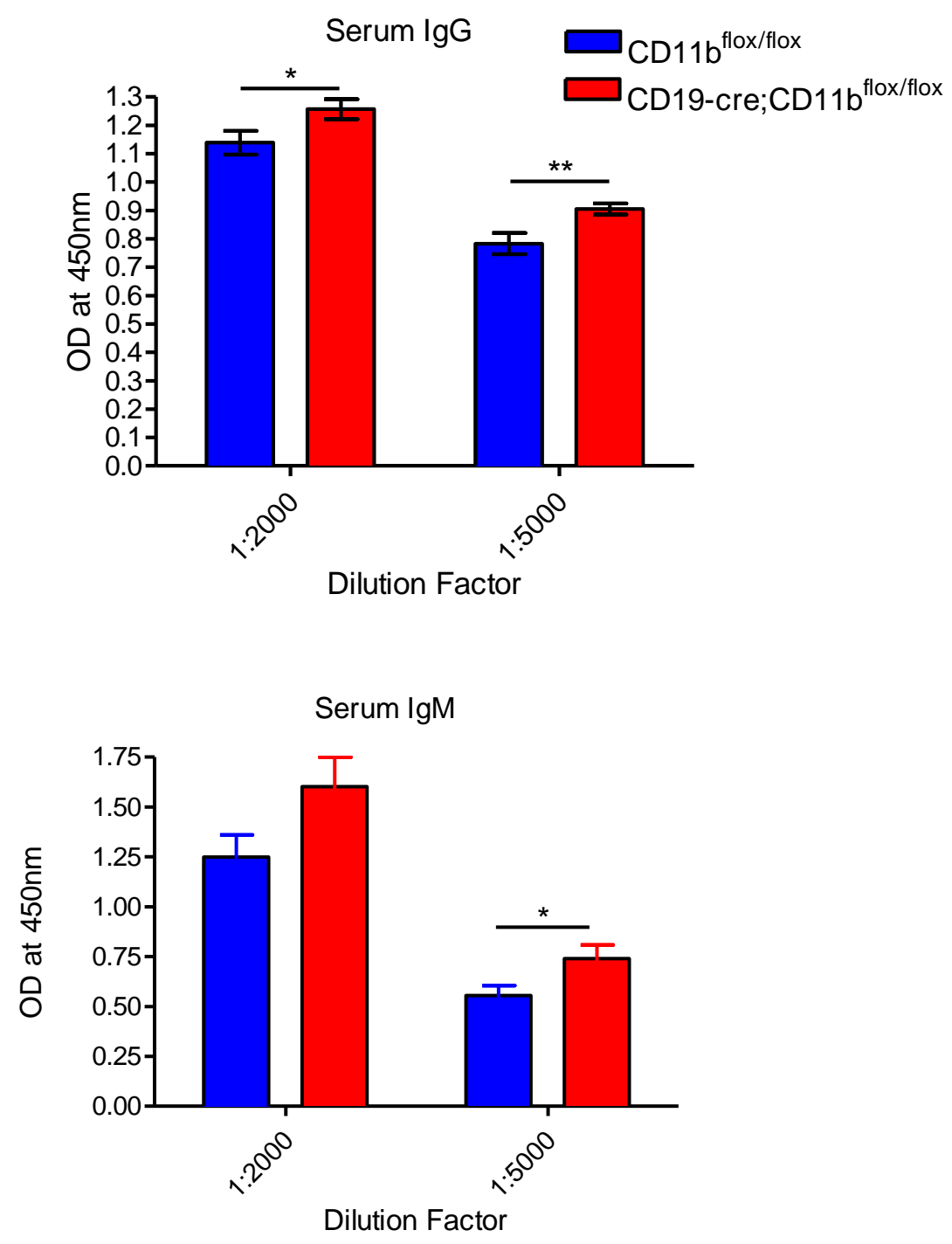

Figure 12: B Cell loss of CD11b leads to increased serum antibody. CD $11 b^{\text {loxp/loxp }}$ and CD11b $b^{\text {loxp/loxp }} ;$ CD19-Cre littermates were immunized 100 $\mu \mathrm{g}$ of OVA suspended in Alum via i.p. injection. Serum was collected on day 14 post-immunization. Anti-OVA IgG and IgM antibody was measured via ELISA at given dilutions in plates coated with $1 \mu \mathrm{g} /$ well of OVA. Optical density was measured at 450nm. Data are means \pm s.e.m. Summarized data are from at least three independent experiments. ${ }^{*} \mathrm{P}<0.05 ; * * \mathrm{P}<0.01$ (unpaired twotailed Student's t-test). 

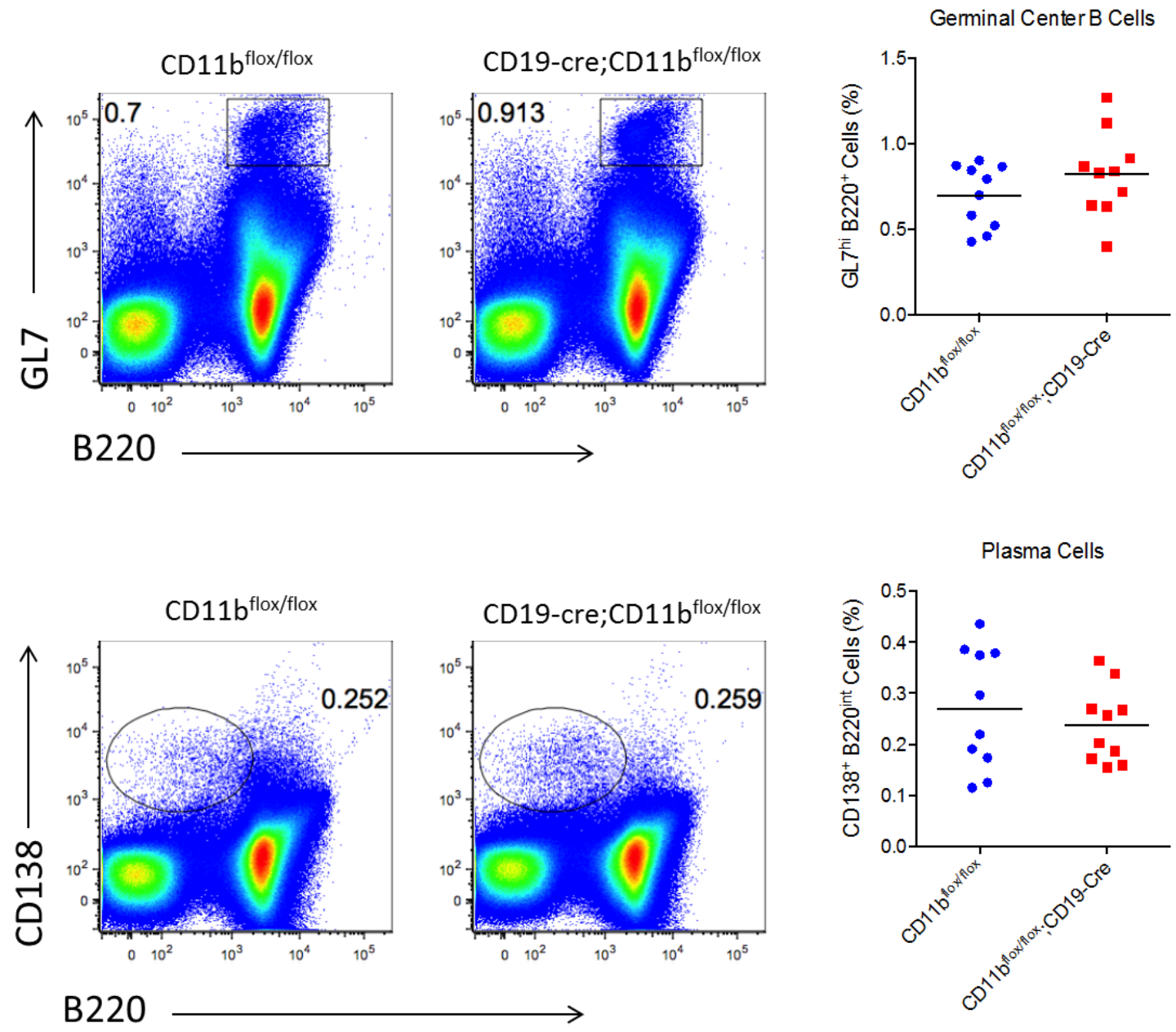

Figure 13: The effect of B cell CD11b deletion on GC and PC formation.

CD11b $b^{\text {loxp/loxp }}$ and CD11b $b^{\text {loxp/loxp}} ;$ CD19-Cre littermates were immunized with $100 \mu \mathrm{g}$ of OVA suspended in Alum via i.p. injection. Spleens were harvested on day 14 postimmunization. Summarized data and representative dot-plots for GCs (top) and PCs (bottom) are shown. Data are means \pm s.e.m. Summarized data are from at least three independent experiments. 


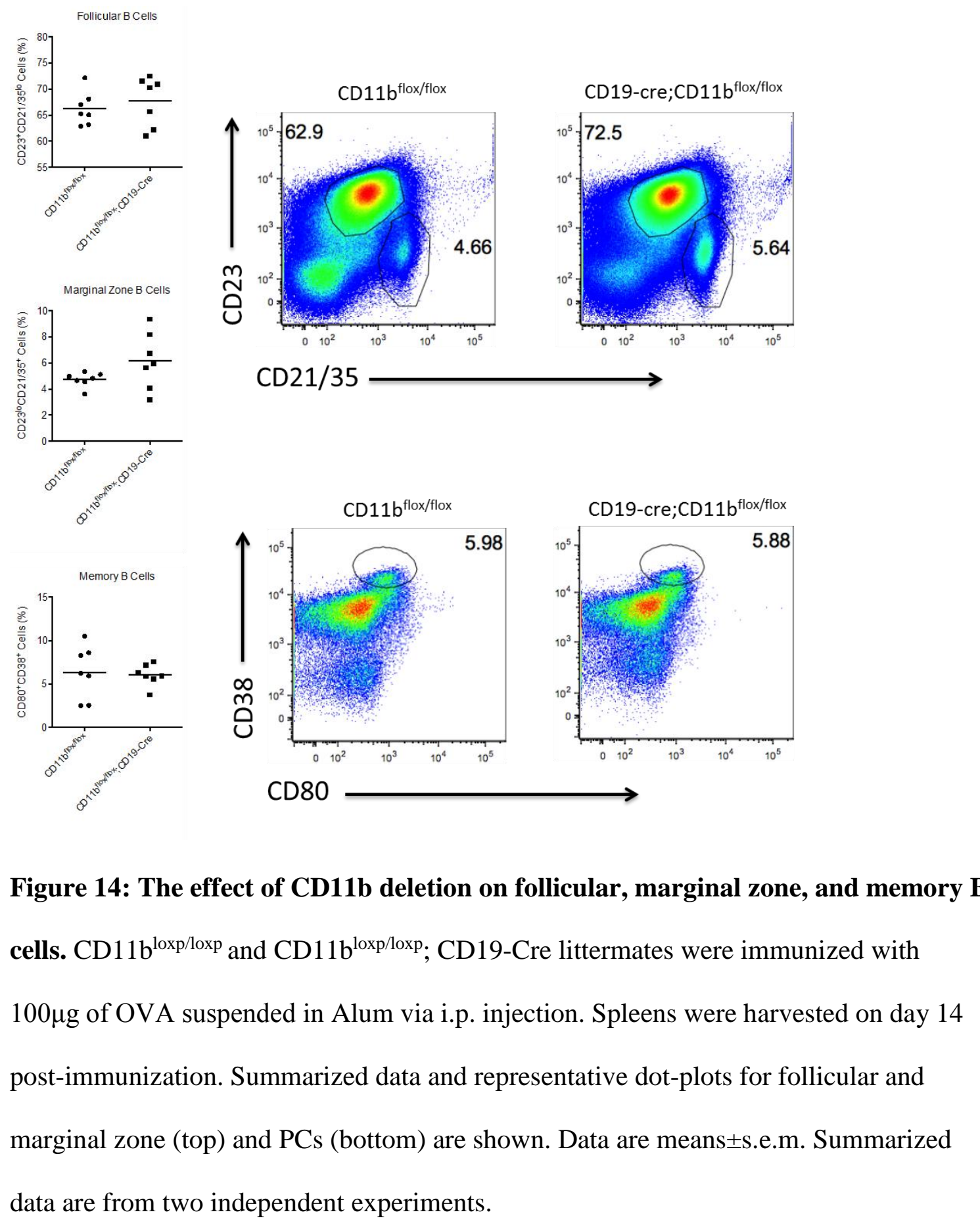




\section{Myeloid cell loss of CD11b does not impact the Th2 antibody response}

With the conditional knockout model established, we used the lysosome 2 (Lyz2) cre system to specifically deplete CD11b expression in monocytes, macrophages, and neutrophils. While the Lyz2 cre model is widely used to study these cell types, its efficiency of deletion is not $100 \%$ and can vary greatly between tissue location and immune setting (155). Thus, we stained for CD11b in CD11b $b^{\text {loxp/loxp }}$, Lyz2-cre mice to determine the knockout efficiency and found a roughly $50 \%$ reduction of CD11b expression on myeloid cells (Figure 15). With this knowledge, we proceeded to examine the effect of this deletion in a Th2 immunization setting. Unlike our previous models, we did not observe any change in the IgM or IgG antibody response (Figure 16). The frequencies of GC and PC cells in the spleen were also unaffected (Figure 17). These results suggest that $\mathrm{B}$ cell expression of $\mathrm{CD} 11 \mathrm{~b}$ controls the heightened immune response observed in global knockout mice, but myeloid cell CD11b does not have a significant impact. 


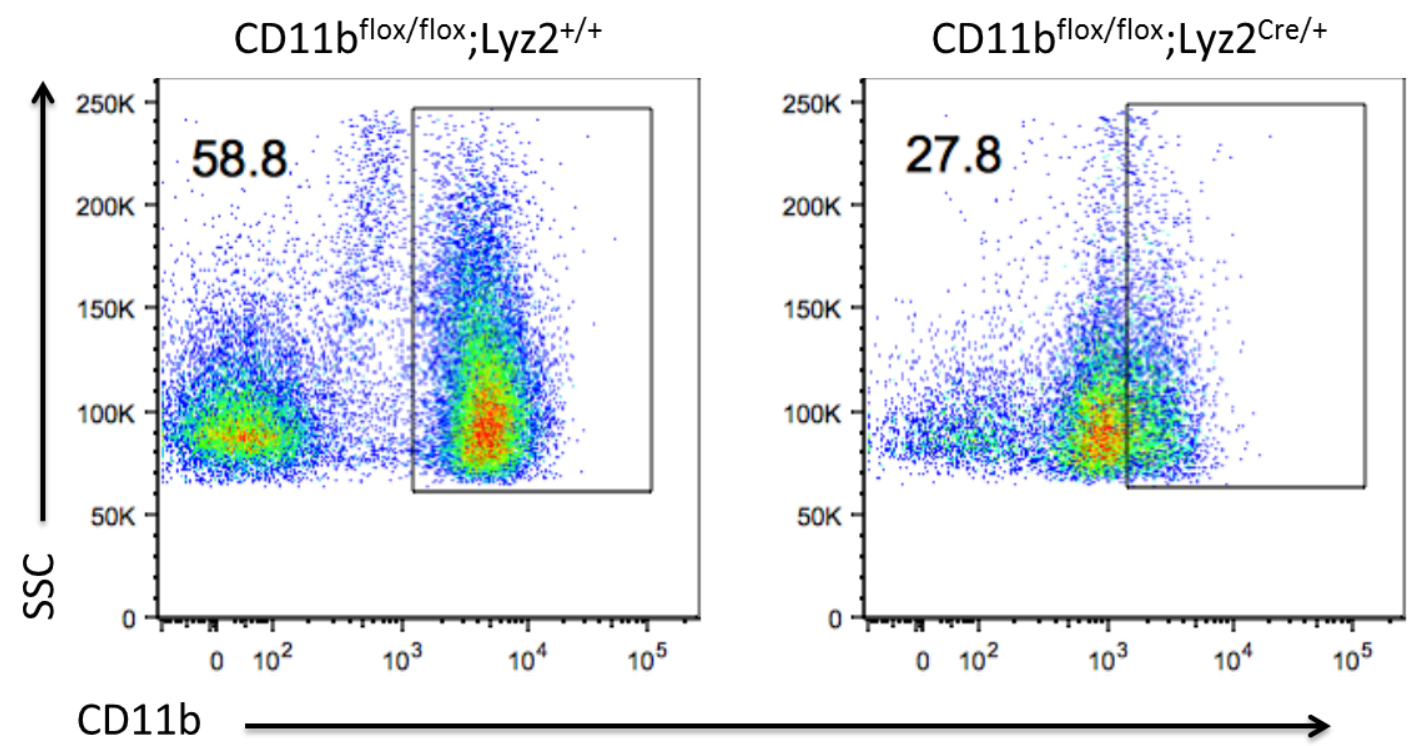

Figure 15: Efficiency of Lyz2-cre driven deletion of CD11b B. Spleens from

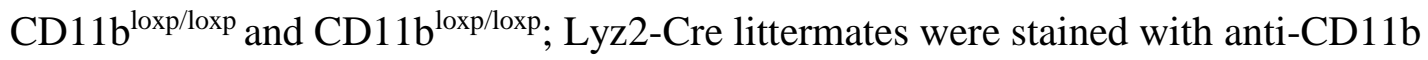
antibody to examine the efficiency of deletion. Representative dot plots are shown. 

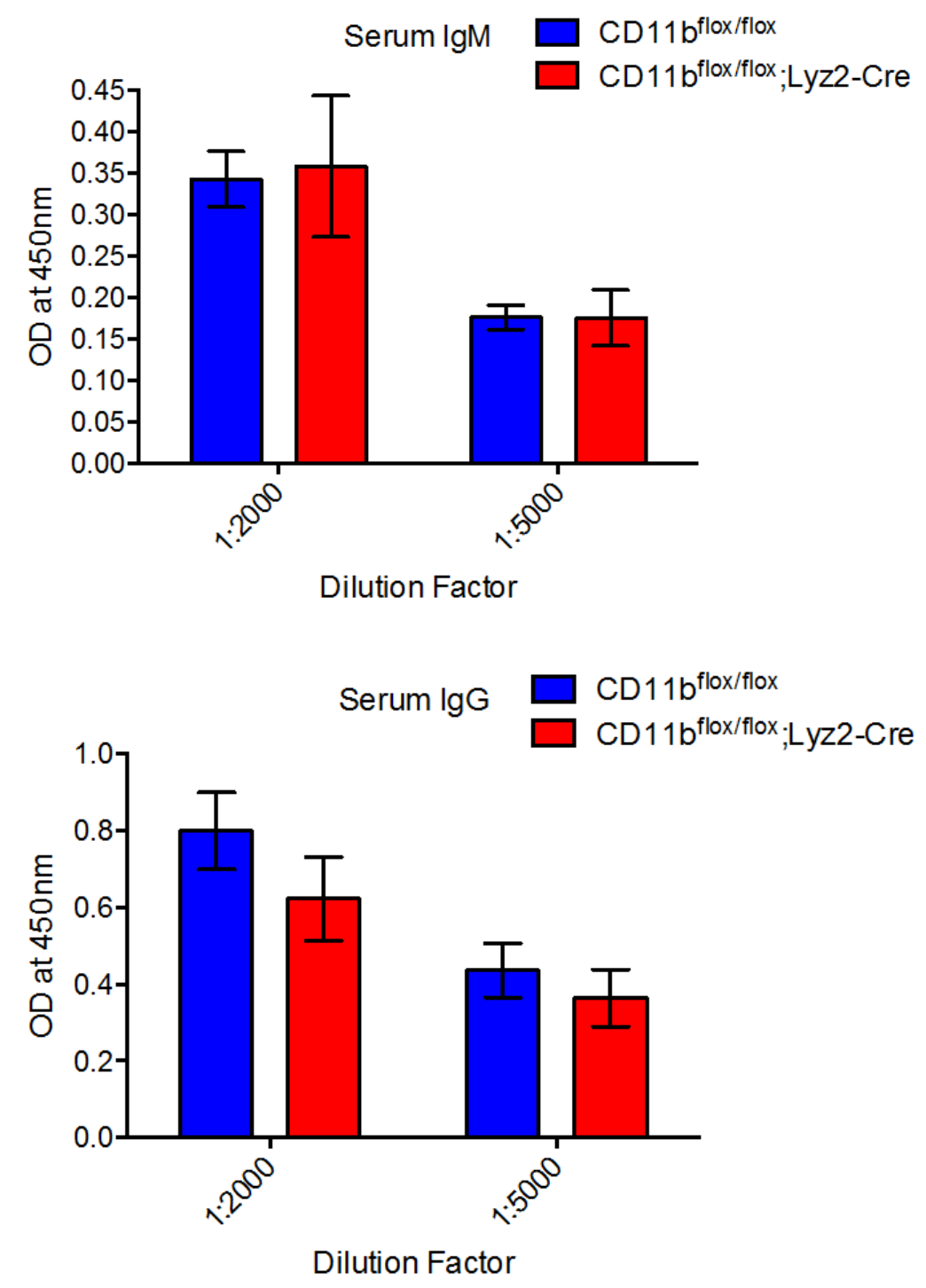

Figure 16: Myeloid cell loss of CD11b does not alter the antibody response.

CD11b ${ }^{\text {loxp/loxp }}$ and CD11b $b^{\text {loxp/loxp}}$; Lyz2-cre littermates were immunized $100 \mu \mathrm{g}$ of OVA suspended in Alum via i.p. injection. Serum was collected on day 14 post-immunization. Anti-OVA IgG and IgM antibody was measured via ELISA at given dilutions in plates coated with $1 \mu \mathrm{g} /$ well of OVA. Optical density was measured at 450nm. Data are means \pm s.e.m. Summarized data are from at least three independent experiments. 

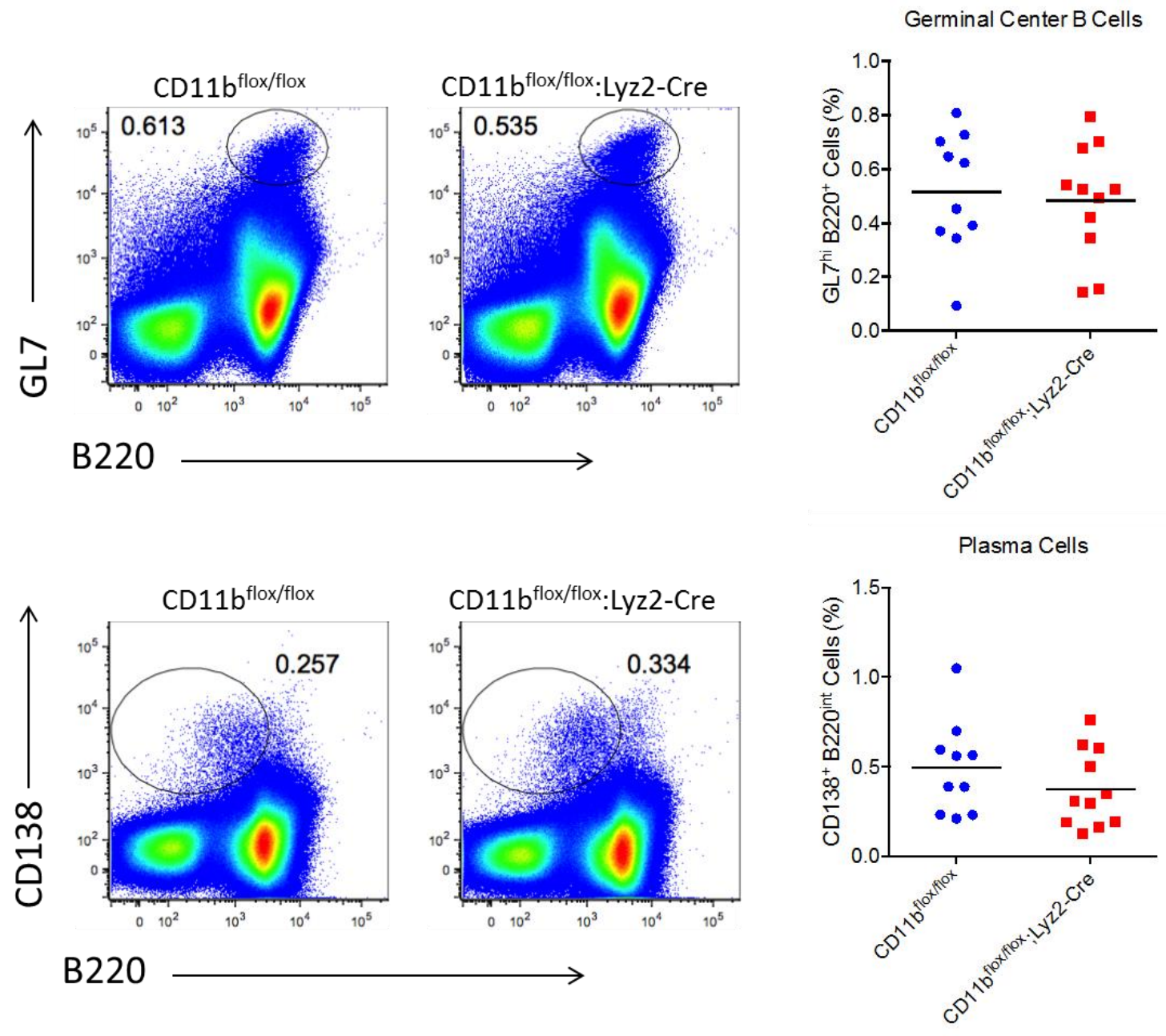

Figure 17: Myeloid cell loss of CD11b does not alter GC and PC differentiation.

CD11b $b^{\text {loxp/loxp }}$ and CD11b $b^{\text {loxp/loxp}}$; Lyz2-cre littermates were immunized with $100 \mu \mathrm{g}$ of OVA suspended in Alum via i.p. injection. Spleens were harvested on day 14 postimmunization. Summarized data and representative dot-plots for GCs (top) and PCs (bottom) are shown. Data are means \pm s.e.m. Summarized data are from at least three independent experiments. 


\section{DISCUSSION}

Our previous in vitro work demonstrated how CD11b negatively regulates naïve B cell activation, while our in vivo studies utilized anti-snRNP transgenic mice and apoptotic cell immunization to demonstrate CD11b's role in preventing autoimmunity. However, the function of $\mathrm{CD} 11 \mathrm{~b}$ as a regulator of the normal immune response was left unknown. As discussed earlier, unique mechanisms and pathways exist to regulate the immune response to different types of stimuli, including autoimmune signals. Whether $\mathrm{CD} 11 \mathrm{~b}$ functions as a general regulator of the $\mathrm{B}$ cell receptor or specialized in autoimmune responses remained to be determined.

In the present work, we utilized a non-autoimmune setting to observe the effect of CD11b on B cells and GC formation in a general immune reaction. Using the NPtg model in a Th2-type immunization allowed us to increase the B cell response without inducing autoimmunity, as well as enabling identification the antigen-specific B cell response (156). We observed an increase in anti-NP IgM and IgG antibody in CD11b deficient mice, suggesting that this effect was not dependent on the autoimmune nature of the previous experiments. While CD11b can function as a negative regulator of downstream antibody-driven autoimmunity, it is not limited to typical autoimmune antigen activation. The increase in both switched IgG and non-switched IgM antibody also shows this regulation impacts antibody resulting from GC B cells as well as extrafollicular B cells. The "choice" between these two maturation pathways is governed by antigen recognition strength, thus implying that B cell CD11b regulation is not dependent on the intensity of BCR-antigen binding (157). Interestingly, we found an increase in the NP-specific GC B cell population of knockout mice, but not in the total or non-NP specific GCs. Contrary to 
this, both NP-specific and non-NP-specific PCs were increased in absence of CD11b. This may be a result of the temporal nature of the GC and PC populations, which persist for roughly 3 weeks and 4 to 6 months, respectively (158). The GC B cells generated by recent NP immunization are limited to this antigen, while end-stage PCs generated by previous antigen responses still persist at a higher level than CD11b sufficient mice. This could be examined in future experiments by sequential immunization with different, traceable antigens and examination of the respective GC and PC populations.

Binding of the BCR promotes expression of survival signals to ensure longevity of the newly activated B cell during subsequent migration and differentiation. BCR ligation induces expression of $\mathrm{NF \kappa B}$, which regulates several transcription factors vital to $\mathrm{B}$ cell development and function. Among them is Bcl-XL, a member of the Bcl-2 family of survival factors. Bcl-XL overexpression in the $\mathrm{GC}$ has been linked to reduced apoptosis and increased survival of weak affinity GC B cells, which leads to excess differentiation to the memory B cell fate (159). Indeed, GCs from our CD11b deficient animals more highly expressed Bcl-XL, likely the result of increased BCR signaling. However, previous studies have demonstrated that $\mathrm{Bcl}-\mathrm{XL}$ overexpression alone does not increase the GC frequency or serum antibody response in an NP immunization model (160). This suggests that additional essential signals are more highly expressed in the absence of CD11b. Bcl-XL expression has also been shown to be vital during plasma cell differentiation in preventing ER stress-induced death (161). Increased expression of Bcl$\mathrm{XL}$ in the PCs of CD11b deficient mice is likely driving increased survival of this population during differentiation. This compounded with the increase in their GC progenitors results in significant increase in total PCs. Blimp-1 was upregulated in 
knockout PCs as well. Expression of Blimp-1 increases as GC B cells downregulate Bcl6 and exit the germinal center to differentiate into PCs, where it continues to increase in expression and drive PC maturity (162). Blimp-1 is also required for PC antibody secretion, suggesting that the $\mathrm{IgG}$ increase seen systemically may also be increased at the individual cell production level (163). Though NFאB can directly bind the Blimp-1 promoter (prdm1), it is not sufficient to induce its transcription alone (164). Additional signals from CD40 and IL-21 engagement are required to fully induce Blimp-1 and PC differentiation (165). Thus, the increase in PC Blimp-1 expression is likely not a direct effect of increased BCR signaling in the absence of CD11b, but rather a downstream consequence of increased GC B cell- $\mathrm{T}_{\mathrm{fh}}$ interaction at the GC stage driven by enhanced BCR signaling.

The BCR signaling cascade acts through and is regulated by phosphorylationcontrolled proteins such as Syk and SHP-1. In this study, we were able to expand upon our previous findings using in vitro B cell line models to demonstrate the effects of CD11b deletion on primary cells ex vivo at the GC B stage. In the absence of stimulation, BCRs cluster into autoinhibitory confirmations. Indeed, our previous work demonstrated CD11b has a stabilizing effect on CD22, which maintains the inhibitory cluster state to control tonic BCR signaling (166). Binding of antigen causes dispersal of these clusters, opening up immunoreceptor tyrosine-based activation motifs (ITAMs) on the cytoplasmic portions of associated CD79 which in turn recruit downstream signals such as Lyn and Syk $(167,168)$. In GC B cells, we did not observe a significant change in pSyk colocalization with BCR following ligation in the absence of CD11b. However, non-GC B cells did demonstrate a defect in pSyk-BCR colocalization in the naïve 
condition, implying pSyk is less important in GC-B cell signaling than non-GCs.

Additionally, a small amount of BCR capping can be seen at the resting state in non-GC knockout cells. Perhaps CD11b is acting as a regulator of tonic signaling at the non-GC stage, producing some BCR cap formation in its absence, but is less important at the GC stage. BCR-pLyn colocalization was decreased in CD11b deficient cells, demonstrating its involvement in maintaining the pLyn negative regulatory circuit at the GC B cell stage. Similarly, SHP-1 recruitment to the BCR region was decreased in the absence of CD11b. These observations confirm CD11b's function in the regulatory circuit of BCR activation at the GC stage, suggesting an impactful role on light-zone affinity-driven selection. Whether it is serving a specific regulatory purpose at this stage or simply retaining its function as a permanent feature of the BCR circuit has yet to be determined. CD11b may be contributing to the high activation levels of SHP-1 that drive dampened BCR signaling within the GC (142). This novel finding of CD11b BCR regulation at the GC stage builds upon our previous work with a naïve B cell line and suggests the possibility of regulatory roles for $\mathrm{CD} 11 \mathrm{~b}$ at other stages of $\mathrm{B}$ cell maturation and development.

The GC reaction is tightly controlled by several transcriptional regulators. Among them are FOXO1 and cMyc. FOXO1 instructs the dark zone side of the reaction and shares many cooperative functions with master GC regulator Bcl6 (145). We observed a decrease in the nuclear localization of FOXO1 following BCR ligation in CD11b deficient cells. In the GC, BCR engagement is restricted to the light zone as newly mutated GC B cells exit the dark zone and compete for uptake of cognate antigen from FDCs. As FOXO1 is limited to the dark zone, its function may be downregulated by 
increased BCR signaling that is further exacerbated by absence of $\mathrm{CD} 11 \mathrm{~b}$ negative regulation. Conversely, cMyc is not expressed in proliferating dark zone GC B cells, and is vital in GC maintenance, formation, and positive selection in the light zone (146). Surprisingly, CD11b deficient cells had significantly less nuclear localization of cMyc at the steady state and after BCR ligation. This may be a result of downstream negative regulatory elements compensating for the increased signaling resulting from CD11b's absence. Alternatively, CD11b may have an unknown function related to the nuclear utilization kinetics of cMyc. Other studies have utilized additional signals present in TfhGC B cell interactions to examine cMyc, such as CD40L. The absence of these signals may be influencing the effects seen here and should be incorporated in future experiments. Despite this, these data outline a role for $\mathrm{CD} 11 \mathrm{~b}$ negative regulation of $\mathrm{BCR}$ signaling at the GC stage of B cell development. Loss of function ITGAM mutations in SLE patients may be contributing to disease by increased BCR activation during this critical process, resulting in increased production of high-affinity antibody-producing PCs.

The generation of CD11b-flox mice allowed us to examine the specific contributions of different cell types to this phenotype. Use of a CD19-Cre model to eliminate B cell CD11b expression was able to replicate the increase in serum IgG and IgM seen in the global knockout, but not the increases in GC and PC frequencies. However, in global CD11b knockout mice, only the NP-specific fraction of GCs was increased while the total was unchanged. The inability to replicate this phenotype in the B cell-specific knockout could be due to the lack of an increased antigen-specific naïve B cell population present in the NP mouse model. Combining the NP model with the tissue- 
specific CD11b knockout could reveal the potential effects of this antigen bias. The lack of increase in the PC population was unexpected, as both NP-specific and total PC fractions were higher in global knockouts. This suggests that B cell CD11b deficiency is not sufficient to increase PC differentiation. However, increases in serum antibody indicate that these PCs are hyperactive, producing greater amounts of $\operatorname{IgG}$ and $\operatorname{IgM}$ at the individual cell level. This can be confirmed with future in-vitro stimulation experiments.

Despite the relatively high populations of CD11b expressing memory B cells found in our reporter model, we did not observe any changes in these subsets in B cell CD11b knockout mice. In memory B cells, CD11b has been shown to be vital to trafficking and migration, especially in the peripheral blood (169). As these cells were of the spleen, loss of CD11b may be less impactful. Additionally, memory B cell generation is still ongoing at 14 days post-immunization. Investigation at the 4 -week timepoint would provide a more complete model of the memory B response $(170,171)$.

Decision between the marginal zone and follicular B cell fate is governed by the strength of BCR signaling (172). A previous study demonstrated deletion of BCR negative regulator $\mathrm{CD} 22$ resulted in a reduction in marginal zone $\mathrm{B}$ cells in favor of the follicular compartment (173). Despite this, we did not observe any changes in these populations in B cell CD11b knockout mice. This suggests that CD11b does not play a significant role in BCR signal regulation at this stage.

CD11b expressing myeloid cells are known to be important in the priming and expansion of B cells during an alum immunization reaction $(174,175)$. Even CD11b expressing myeloid-derived suppressor cells (MDSCs) have been shown to enhance B cell antibody production in certain settings $(176,177)$. Interestingly, CD11b expressing 
myeloid cells were found to suppress lupus-like disease in male, but not female, NZB mice (178). Depletion of CD11b expressing neutrophils can even cause accelerated disease in both genders of these mice (179). However, usage of myeloid cell expressed lysosome-cre driven conditional knockout was unable to replicate any of the enhanced B cell response phenotypes seen in our global knockout animals. In these cells, most notably macrophages and neutrophils, CD11b functions in cell-cell adhesion and trafficking to sites of inflammation. Our results imply that defects in this mechanism are not sufficient to alter the ability of these cells to enhance the B cell response, and perhaps they maintain adequate trafficking through other pathways. Marginal zone macrophages are essential for B cell follicle trafficking and GC formation, but this subset expresses minimal levels of CD11b and is likely unaffected by deletion or mutation $(180,181)$. Alternatively, the non-significant changes in these mice may be due to the inefficiency of the lysosome-cre knockout model, which was only able to reduce expression by an average of $50 \%$.

These experiments have demonstrated that $\mathrm{CD} 11 \mathrm{~b}$ is able to regulate the humoral response in a B cell intrinsic manner. We have revealed novel BCR signaling regulation by CD11b at the GC B cell stage and its downstream effect on antibody production (Figure 18). This data suggests a widespread function for $\mathrm{CD} 11 \mathrm{~b}$ regulations of the $\mathrm{BCR}$ across different $\mathrm{B}$ cell subsets and warrants the investigation of its potentially novel role in these different populations. 


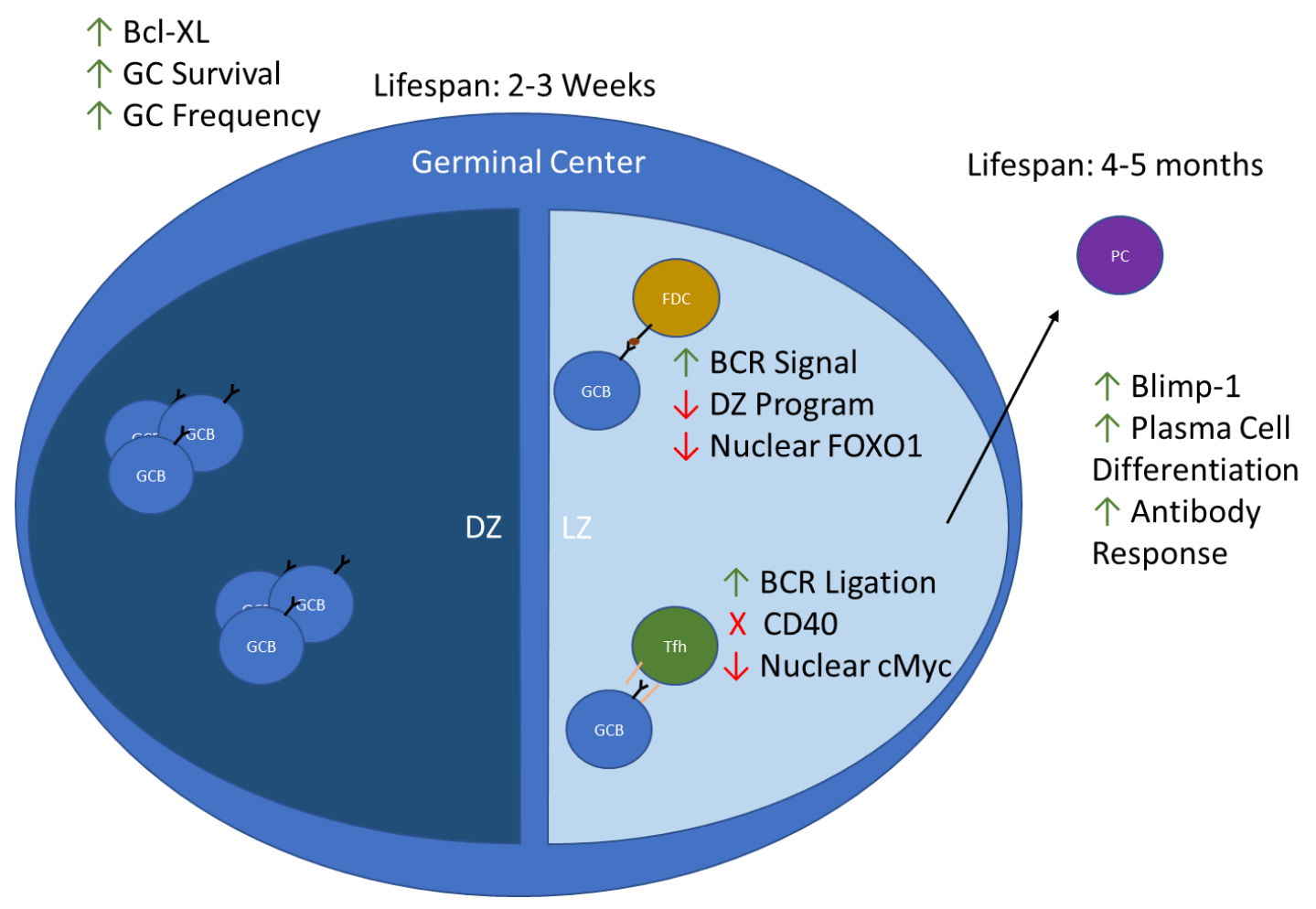

Figure 18: Loss of CD11b alters GC B cell signaling and regulation and enhances the humoral response. 
CHAPTER II

DYNAMIC EXPRESSION OF CD11B ON B CELLS IN NAÏVE AND IMMUNE SETTINGS 


\section{INTRODUCTION}

The identification of CD11b as a regulator of B cell BCR signaling at the GC and naïve B cell stages has revealed the possibility of roles in other B cell subsets as well. Preventing unwanted stimulation of B cells via BCR negative regulation is vital at every step of B cell development. However, mechanisms of regulation are dynamic. As discussed in the previous chapter, various surface BCR co-receptors specialize in negative regulation in response to specific ligands, such as CD72 and Sn/RNP. The varying necessities of B cells at different stages of maturity may require more or less regulation from a given receptor mechanism. Whether regulation by CD11b has similar restrictions remains to be determined. To effectively study $\mathrm{CD} 11 \mathrm{~b}$ regulation of the $\mathrm{BCR}$, its expression on different B cell subsets must be examined.

\section{BCR Signaling In B Cell Differentiation}

As the hallmark receptor of B cells, the BCR acts as an important signaling pathway at all stages of development and differentiation. Understanding of the BCR's function at various B cell stages is necessary to better examine the potential role of $\mathrm{CD} 11 \mathrm{~b}$ as a negative regulator. Expression of the BCR begins in the early development within the bone marrow, as immature B cells express the pre-BCR which features a $\mathrm{V}(\mathrm{D}) \mathrm{J}$ recombined heavy chain and a temporary surrogate light chain (182). Even this pre-BCR stage engages in ligation and activation pathways to drive selection of functional heavy-chains (183). After the addition of a recombined light chain, BCR signaling is once again vital to filter out autoreactive B cells from the newly formed repertoire (184). By the mature B cell stage, expression of the IgM-BCR is subject to activation and negative regulation via previously discussed pathways. The IgD-BCR can 
also be expressed in these cells. The molecular pathways involved in $\operatorname{IgD}$ activation and negative regulation have been shown to differ from that of IgM, but overall remain poorly understood (139). BCR signaling strength governs the differentiation between marginal zone and follicular B cells fates, with higher levels of signal favoring the latter (185). At the follicular stage, antigen encounter via the BCR enhances activation and drives migration to the $\mathrm{T}$ zone to receive T cell signal $(186,187)$. After T-B interaction, B cell fate is governed by affinity. BCRs with high affinity have stronger BCR signaling and receive more $\mathrm{T}$ cell help, driving them to extrafollicular plasma cell differentiation (157). As discussed in the previous chapter, BCR signaling in the GC is minimal due to high activation of negative regulator SHP-1. B cells with lower yet still competitive affinity enter the GC. Regulation of the BCR signal is crucial during all of these processes to ensure a proper B cell response that prevents the unwanted activation of offtarget cells yet promotes the correct fate for needed effectors. Our identification of $\mathrm{CD} 11 \mathrm{~b}$ as a novel BCR regulator reveals the need to re-examine its presence in different B cell subsets.

\section{Known Roles of CD11b in B Cells}

Despite its classic association with myeloid cells, CD11b expression has been identified on B cells for many years (188). However, the function and purpose of B cell CD11b is still not well established. One initial study to examine these cells found that $\mathrm{CD} 11 \mathrm{~b}$ is expressed more on $\mathrm{CD}^{2} 7^{+} \mathrm{IgD}^{-}$memory $\mathrm{B}$ cells than other peripheral $\mathrm{B}$ cell populations. These cells were demonstrated to migrate in vitro more effectively than

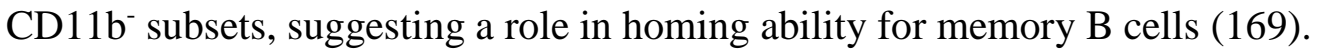


A small subset of B1 B cells, roughly 10\%, expresses CD11b. These cells were found to specialize in stimulation and expansion of $\mathrm{CD}^{+} \mathrm{T}$ cells and produced far less IgM than the majority $\mathrm{CD} 11 \mathrm{~b}^{-}$subset. In SLE patients, the $\mathrm{CD} 11 \mathrm{~b}^{+}$population is increased three-fold and have enhanced T cell stimulation activity (189). These same cells have also been shown to produce IL-10 and suppress T cells activation, demonstrating a two-fold role in regulation of T cells (190). In mice, B1 CD11b expression has been suggested as an indicator of the development stage (191). IL-10 dependent $\mathrm{CD} 11 \mathrm{~b}^{+} \mathrm{B}$ cells have also been described as $\mathrm{T}$ cell regulators in autoimmune hepatitis mice (192).

A CD11b expressing population of plasma cells has also been described. These intestinally-located plasma cells reside in the Peyer's patch, and produce higher amounts of IgA antibody than $\mathrm{CD}_{11} \mathrm{~b}^{-}$cells. They also proliferate more rapidly and are dependent on IL-10. These CD11b plasma cells are important for early IgA responses in the intestine following oral immunization (193).

CD11b (also known as complement receptor 3 (CR3)) expression in $\operatorname{IgM}^{+}$splenic B cells was found in relation to complement component 3 (C3). In C3 deficient animals, previously unseen expression of CD11b was found to be 2 to three-fold higher than $\mathrm{C} 3$ sufficient mice (194). This was determined to be an effect driven by lack of environmental C3, as B cells from wild-type animals began to express more CD11b after transfer into $\mathrm{C}^{-/-}$mice. These findings describe a role for $\mathrm{C} 3$ in controlling the expression of $\mathrm{CD} 11 \mathrm{~b}$ on B cells, though the purpose of this function is still unclear.

Despite these known CD11b expressing B cell populations, the role of CD11b in B cells remained largely unstudied. Demonstration of CD11b as a novel BCR regulator 
requires the re-examination of CD11b in these previously described subsets. The presence of CD11b across B cell subsets must also be re-evaluated given the implication of these new findings on immune regulation.

In this study, we examine CD11b expression on various B cell subsets using an endogenous CD11b-GFP reporter. We identify previously unreported populations of CD11b expressing cells. Interestingly, these expression patterns change during the immune state, revealing a dynamic role for $\mathrm{CD} 11 \mathrm{~b}$ in $\mathrm{BCR}$ regulation. $\mathrm{CD} 11 \mathrm{~b}$ expression in Fas ${ }^{\mathrm{lpr}}$ autoimmunity is also examined. In these animals, B cell CD11b patterns are altered with disease progression, and fundamentally changed from the naïve and healthy immune settings. 


\section{MATERIALS AND METHODS}

Mice

CD11b reporter (FRT) mice were generated for the purpose of this study. Lupus-prone CD11b reporter Fas ${ }^{\text {lpr/lpr }}$-FRT mice were generated by interbreeding FRT mice with Fas ${ }^{\text {lpr/pr }}$ mice (The Jackson Laboratory). Lupus-prone CD11b reporter ABIN1-FRT mice were generated by interbreeding FRT mice with ABIN1[D485N] inactive ABIN1 knockin mice (a gift from Dr. David Powell). Mice at 6-8 weeks of age with both sexes were used for immunization experiments. Aged mouse experiments were used at the indicated timepoints. All mice were housed and bred in a conventional facility at the University of Louisville. Animal care and experiments were conducted in accordance with the National Institutes of Health guidelines and were approved by the Institutional Animal Care and Use Committee at the University of Louisville.

\section{Immunizations and Ab Detection}

FRT-reporter mice were immunized via intraperitoneal (i.p.) injection of $100 \mu \mathrm{g}$ OVA peptide in Alum adjuvant at a 1:1 ratio. Sera was collected before and at given timepoints after immunization, or at noted time-points in aged mice. Anti-OVA and antibody titers were measured via enzyme-linked immunosorbent assay (ELISA) using a 96-well plate coated with $1 \mu \mathrm{g}$ of OVA and a standard ELISAMAX kit (Biolegend). Mouse antidsDNA antibody was detected using an anti-dsDNA ELISA kit (Signosis Inc).

Antibodies, Flow Cytometry, and Cell Sorting 
Single-cell suspensions were obtained from spleen or bone marrow and treated with Fcblocker for 10 minutes on ice followed by staining with appropriate fluorochrome-labeled antibody or isotype controls for at least 20 minutes at $4^{\circ} \mathrm{C}$. Cells were then washed with 10 volumes of PBS before resuspension in PBS for acquisition or Running Buffer (Miltenyi Biotech) for sorting. For intracellular staining, cells were fixed and permeabilized in appropriate buffers (Biolegend) followed by the addition of fluorochrome-labeled antibody. Cells were then washed two times with 10 volumes of permeabilization buffer and resuspension for acquisition. Flow cytometry samples were acquired using a FACSCanto cytometer (BD Biosciences) and analyzed using FlowJo software (Tree Star). Sorting was performed using FACSAria III (BD Biosciences) or MoFlo XDP (Beckman Coulter). Cell purity was confirmed at greater than $90 \%$ via flow cytometry. The following anti-mouse antibodies were used: Gr-1, NK1.1, IgD, IgM, GL7, B220 (CD45R), CD3, CD11b, CD11c, CD21/35, CD23, CD38, CD43, CD80, CD138 (Biolegend).

\section{BCR Repertoire Sequencing}

Sort-purified cells were frozen in TRIzol (Life Technologies) for lysis and storage. RNA isolation was performed using chloroform extraction and an RNEasy Micro Kit (Qiagen).

\section{Statistical Analysis}

All quantitative data are shown as mean \pm s.e.m unless otherwise indicated. All samples were compared using two-tailed, unpaired Student's $T$ test. A $P$ value less than 0.05 was considered significant. Statistical analysis was performed with Prism (Graphpad 
Software). 


\section{B Cell CD11b expression is altered in a Th2 immunization setting.}

Our previous data has clearly defined an effect of CD11b expression on the naïve B, GC B, and plasma cell stages. However, expression of CD11b at each stage of B cell development is not well characterized. In order to examine this, we generated a CD11breporter mouse model, described as "FRT", which adds a GFP tag to the CD11b promoter site (Figure 19). Though CD11b expression on B cells has been demonstrated to be very low, this system allows for a more sensitive quantification directly controlled by transcription and expression of $\mathrm{CD} 11 \mathrm{~b}$ rather than traditional antibody staining which can be subject to nonspecific binding effects.

We first sought to investigate any changes in CD11b expression in an immunization setting compared to a naïve state. A Th2-type immunization was used to build upon our previous studies using global CD11b knockout mice. Beginning at the early stages of development, we examined the pre-pro B, pro B, pre B, and immature B cell populations in the bone marrow. Expression was greatest at the earliest pre-pro B cell stage, and increased even further after immunization (Figure 20). In the intermediate pro $\mathrm{B}$ and pre $\mathrm{B}$ stages, the $\mathrm{CD} 11 \mathrm{~b}$ expressing fraction was minimal, averaging less than even $1 \%$ of the total population (Figure 21). By the immature B cell phase, expression elevated slightly, and was once again increased in the immune setting. As a whole, the developmental stages of B cells have minimal levels of CD11b expression compared to other subsets. Thus, it is unlikely that CD11b is playing a significant role in regulation of their pre-BCR and BCR signaling.

In the peripheral lymphoid organs, we revealed CD11b expression in the follicular and marginal zone B cells of the spleen. The follicular B compartment revealed a low but 
very well-defined population of CD11b expressing cells (Figure 22). Interestingly, expression decreased by nearly half after immunization. The marginal zone CD11 $\mathrm{b}^{+}$ subset was larger, and also showed a similar decrease in immunized mice. Though the marginal zone CD11b expressing frequency is higher than that of the follicular B cell subset, it is less well defined against the CD11b-negative population. Also within the spleen, we examined GC B cells to reveal an average of $2 \%$ of naïve GC B cells express CD11b which decreased by nearly half following immunization (Figure 23). Due to the relatively low frequency of GC B cells, the CD11b expressing subset is very rare and poorly-defined compared to larger populations of B cells. Post-GC long-lived memory B cells reside in the spleen as well. Naïve levels of CD11b in these cells increased dramatically after Th2 immunization, though with some variation (Figure 24). The presence of $\mathrm{CD} 11 \mathrm{~b}$ on memory $\mathrm{B}$ cells is unsurprising given previous reports, but the discovery of CD11b expressing populations within the follicular, marginal zone, and GC compartments is novel. Though the frequency is low, these subsets are easily distinguished from the $\mathrm{CD} 11 \mathrm{~b}^{-}$population and may be playing a key role in regulation of the BCR in these cells.

Among the B cell subsets examined, the plasma cells carry the most consistently high amounts of CD11b expression. An average of $12 \%$ of splenic-resident short-lived plasma cells express CD11b in the naïve state, which decreased after immunization (Figure 25). Surprisingly, the opposite effect was observed in bone marrow-resident longlived plasma cells. Here, naïve CD11b expression averaged at roughly $8 \%$ and increased after immunization (Figure 26). These findings build upon the previous identification of CD11b expressing plasma cells in the Peyer's patch. However, the opposing shift in 
expression under immunization conditions indicates a differential role for CD11b in these two closely related but distinct populations. The varying changes in expression across all of these B cell subsets outlines a complex and dynamic role for CD11b regulation. 


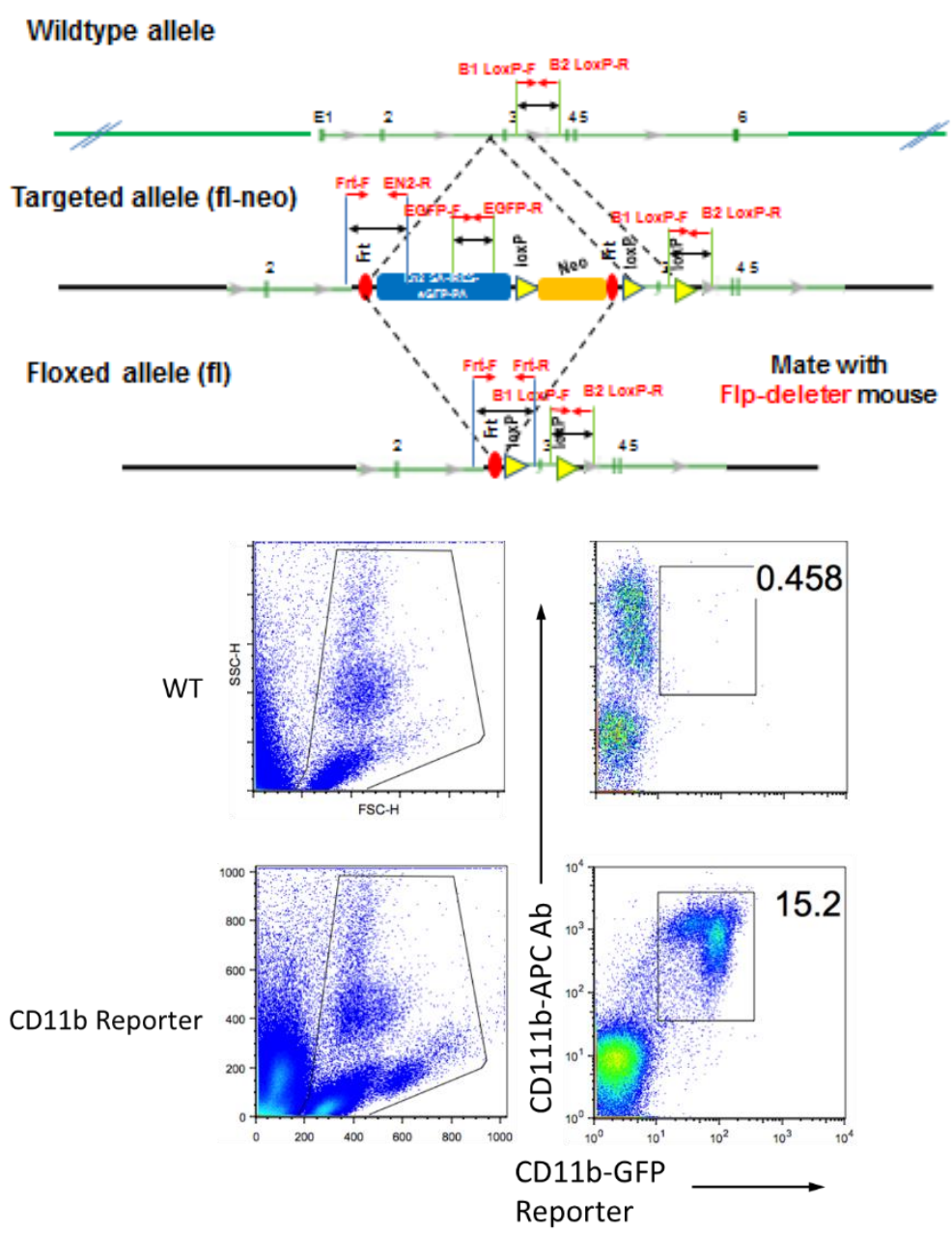

Figure 19: Generation of CD11b-Reporter mice. CD11b reporter mice were generated for this purpose of this study using. Construct design (top). Briefly, GFP was inserted into the promoter region of the third exon of CD11b using a loxp-neo cassette. To verify reporter effectiveness, spleen cells from FRT mice were stained with CD11b-APC antibody and compared against the FITC channel reporter (bottom). 


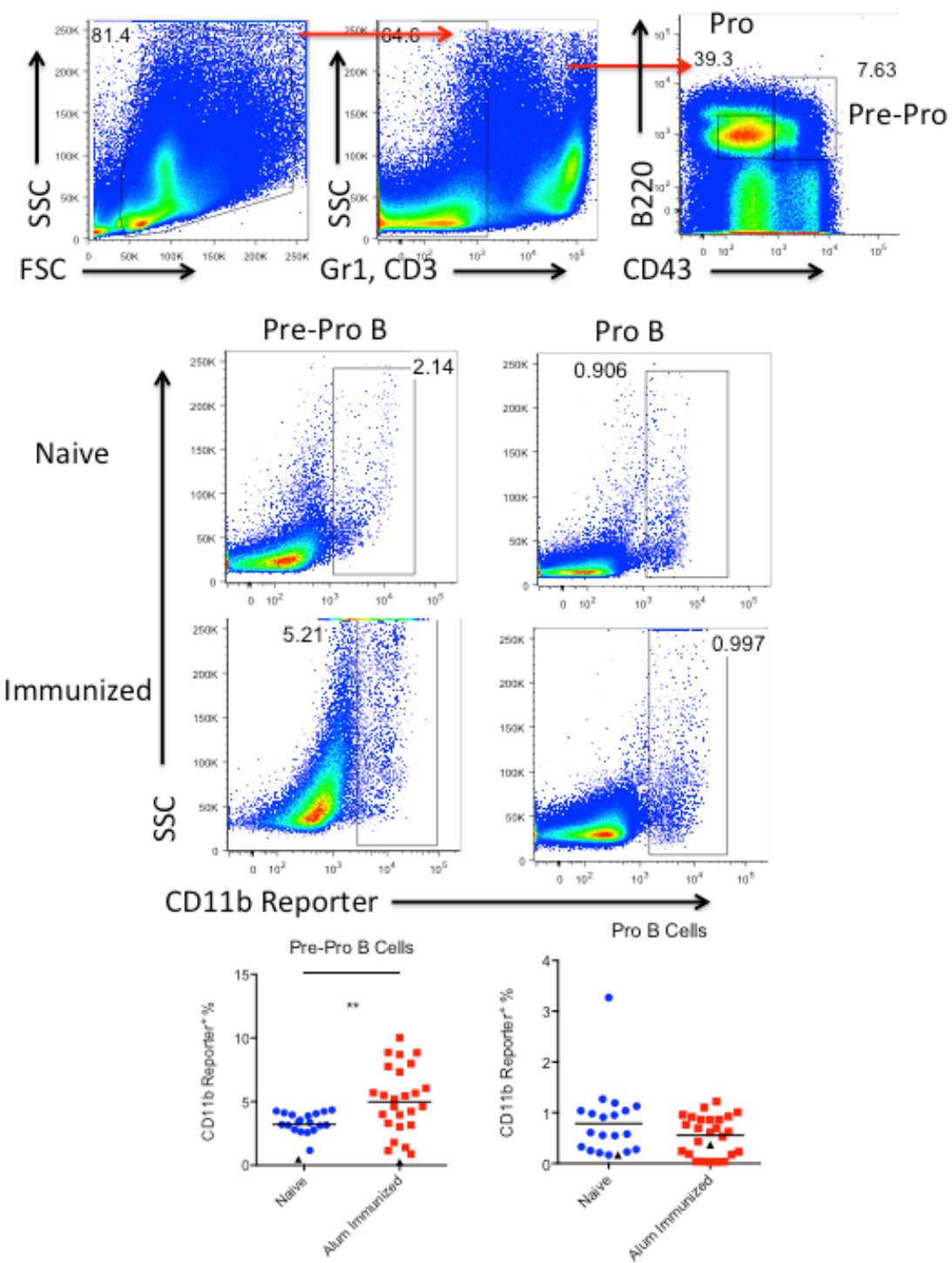

Figure 20: The impact of immunization on CD11b expression in early-stage developing B cells of the bone marrow. FRT mice were immunized with $100 \mu \mathrm{g}$ of OVA suspended in Alum via i.p. injection. Bone marrow was harvested on day 14 postimmunization alongside naïve littermates. Gating strategy for identifying pre-pro and pro B cells (top) and representative dot-plots for CD11b expression (bottom) are shown.

Reporter-negative littermates were used as referential controls, shown by $\boldsymbol{\Delta}$. Summarized data are from at least three independent experiments. $* * \mathrm{P}<0.001$ (unpaired two-tailed Student's t-test). 

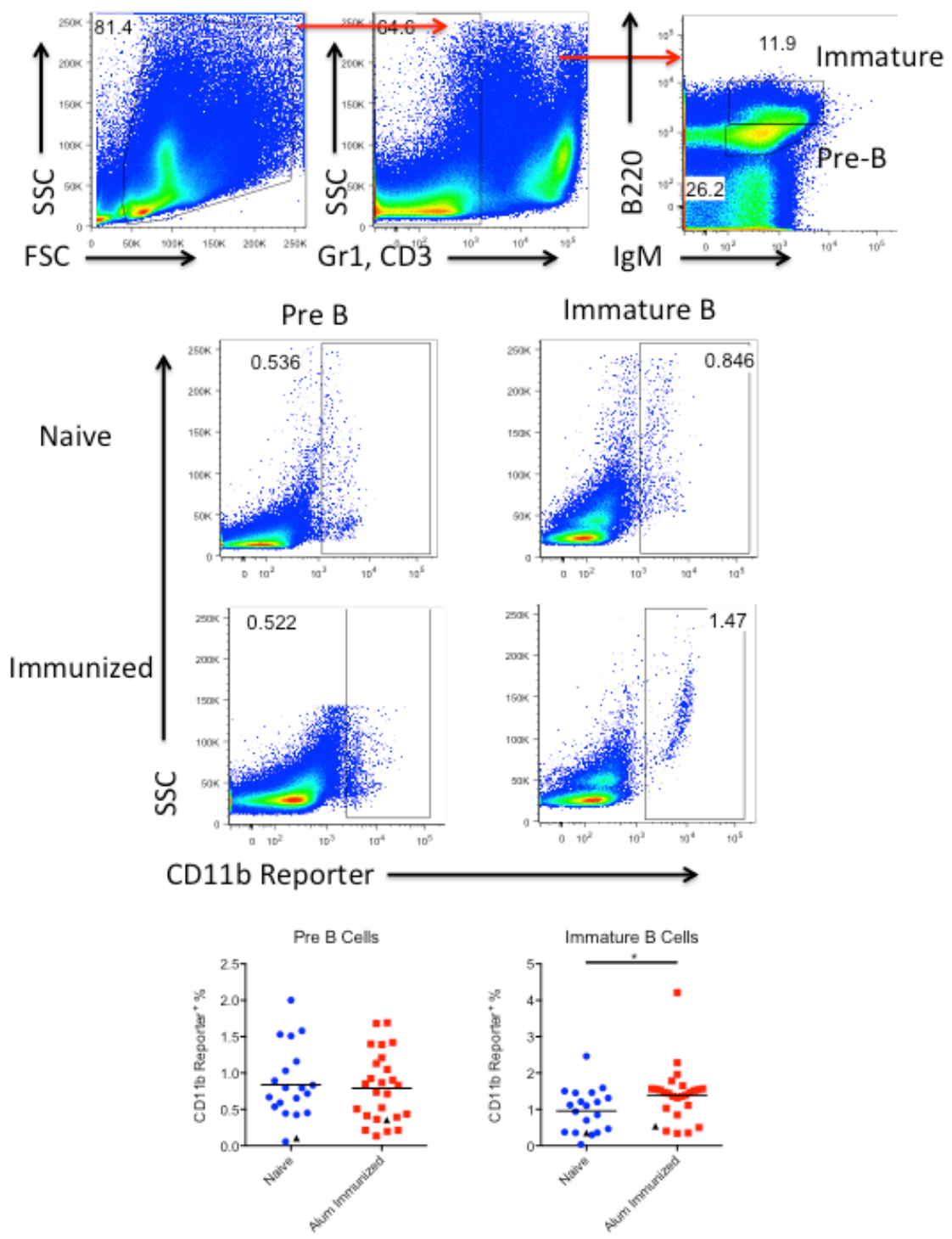

Figure 21: The impact of immunization on CD11b expression in later-stage

developing B cells of the bone marrow FRT mice were immunized with $100 \mu \mathrm{g}$ of OVA suspended in Alum via i.p. injection. Bone marrow was harvested on day 14 postimmunization alongside naïve littermates. Gating strategy for identifying pre B and immature B cells (top) and representative dot-plots for CD11b expression (bottom) are shown. Reporter-negative littermates were used as referential controls, shown by $\boldsymbol{\Lambda}$. Summarized data are from at least three independent experiments. $* \mathrm{P}<0.05$ (unpaired two-tailed Student's t-test). 

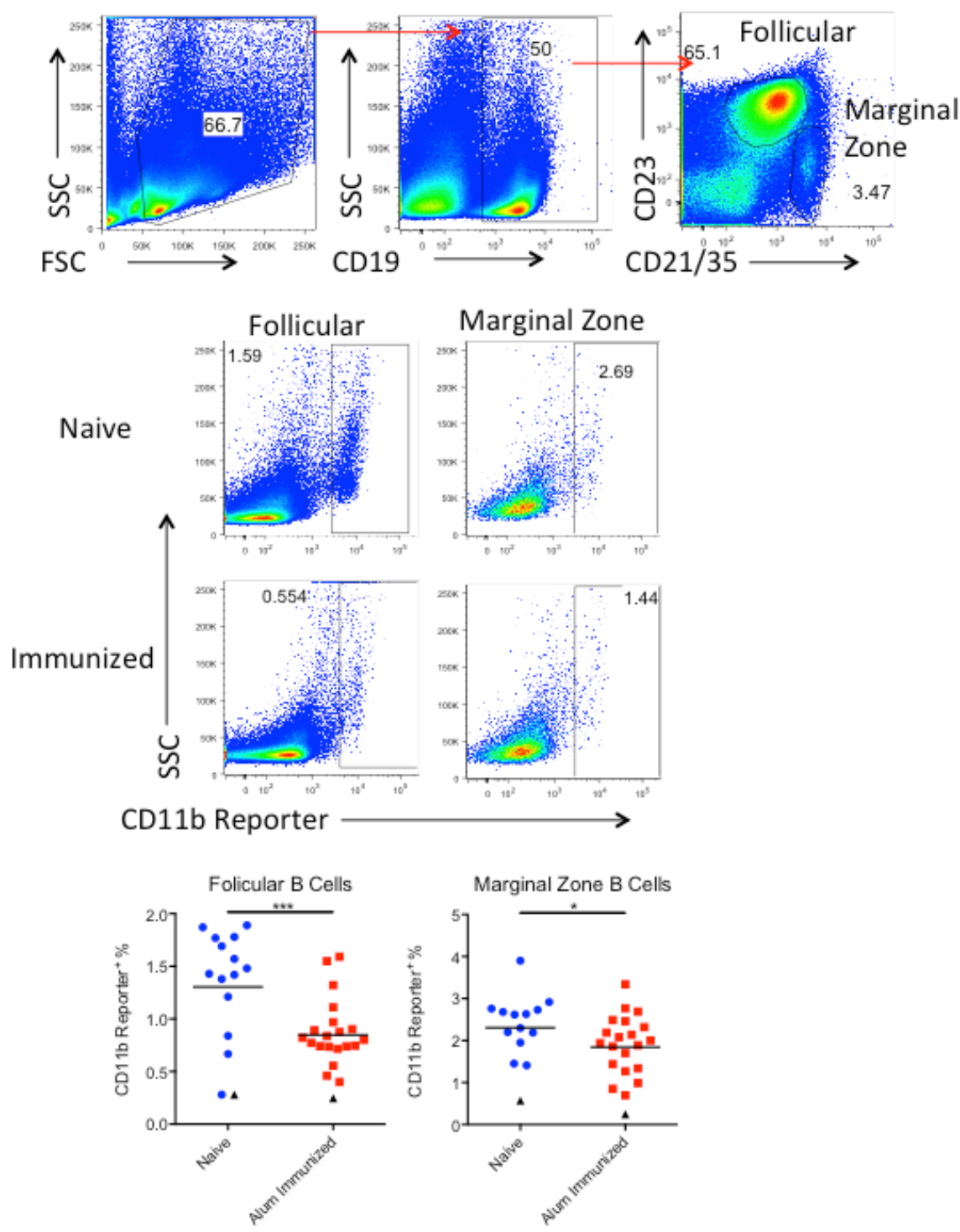

Figure 22: The impact of immunization on CD11b expression in splenic marginal zone and follicular B cells. FRT mice were immunized with $100 \mu \mathrm{g}$ of OVA suspended in Alum via i.p. injection. Spleens were harvested on day 14 post-immunization alongside naïve littermates. Gating strategy for identifying marginal zone and follicular B cells (top) and representative dot-plots for CD11b expression (bottom) are shown.

Reporter-negative littermates were used as referential controls, shown by $\boldsymbol{\Lambda}$.

Summarized data are from at least three independent experiments. $* \mathrm{P}<0.05$, $* * \mathrm{P}<0.0001$ (unpaired two-tailed Student's t-test). 

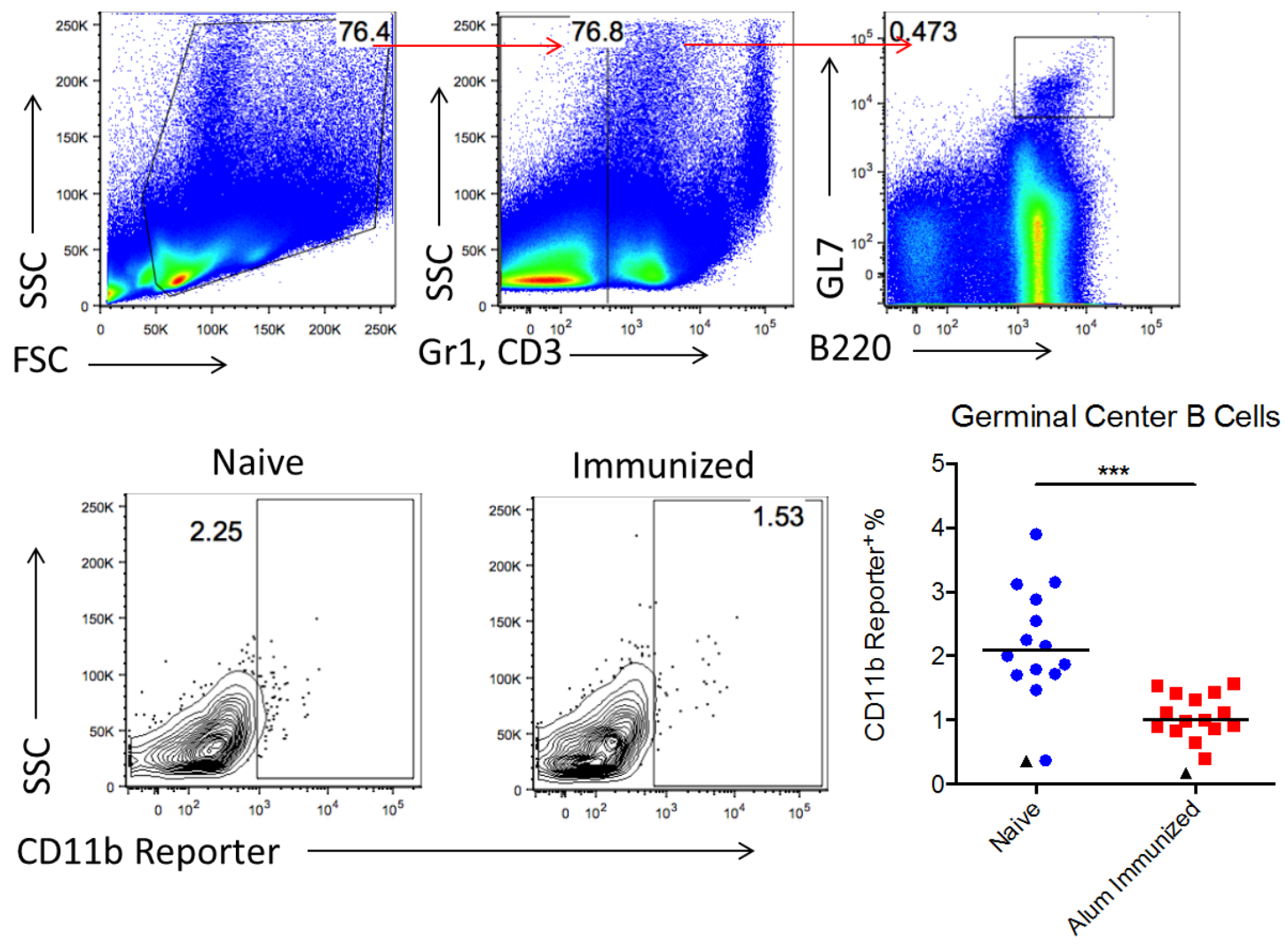

Figure 23: The impact of immunization on CD11b expression in splenic GC B cells.

FRT mice were immunized with $100 \mu \mathrm{g}$ of OVA suspended in Alum via i.p. injection.

Spleens were harvested on day 14 post-immunization alongside naïve littermates. Gating strategy for identifying GC B cells (top) and representative dot-plots for CD11b expression (bottom) are shown. Reporter-negative littermates were used as referential controls, shown by $\boldsymbol{\Lambda}$. Summarized data are from at least three independent experiments. $* * * \mathrm{P}<0.0001$ (unpaired two-tailed Student's t-test). 

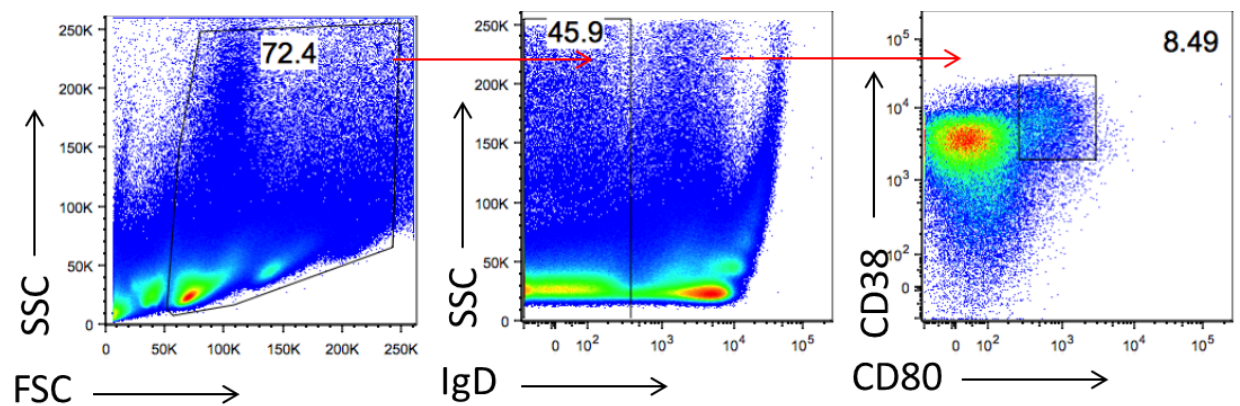

FSC

IgD CD80
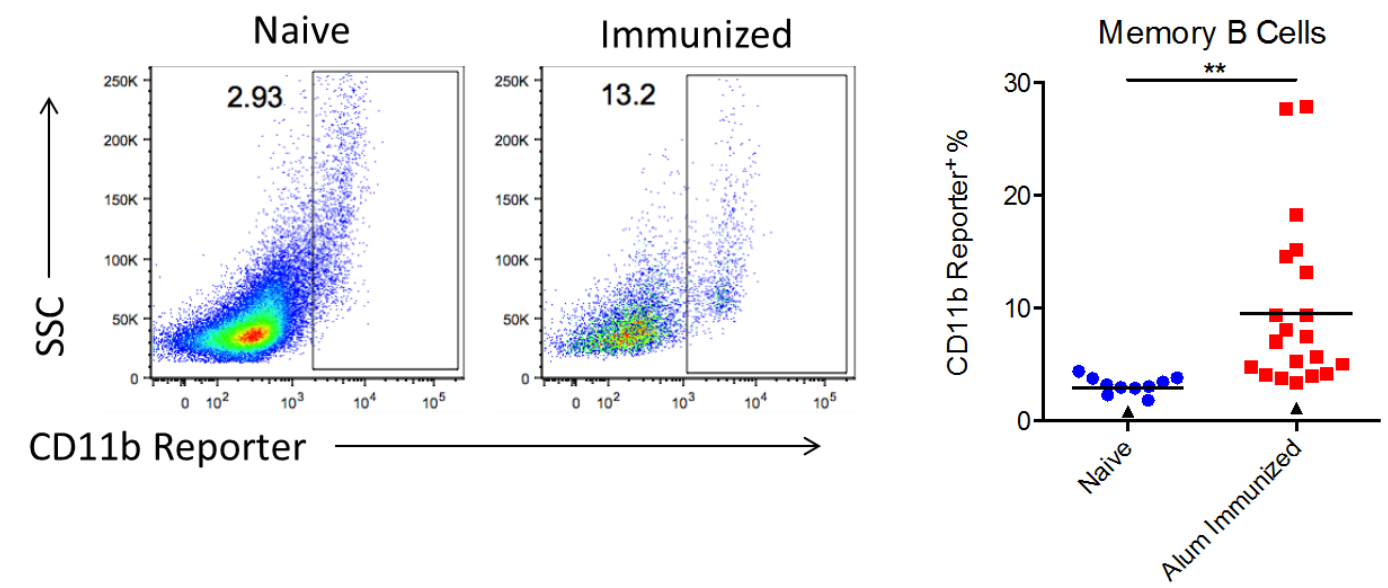

Figure 24: The impact of immunization on CD11b expression in splenic memory B cells. FRT mice were immunized with $100 \mu \mathrm{g}$ of OVA suspended in Alum via i.p. injection. Spleens were harvested on day 14 post-immunization alongside naïve littermates. Gating strategy for identifying memory B cells (top) and representative dotplots for CD11b expression (bottom) are shown. Reporter-negative littermates were used as referential controls, shown by $\boldsymbol{\Lambda}$. Summarized data are from at least three independent experiments. $* * \mathrm{P}<0.001$ (unpaired two-tailed Student's t-test). 

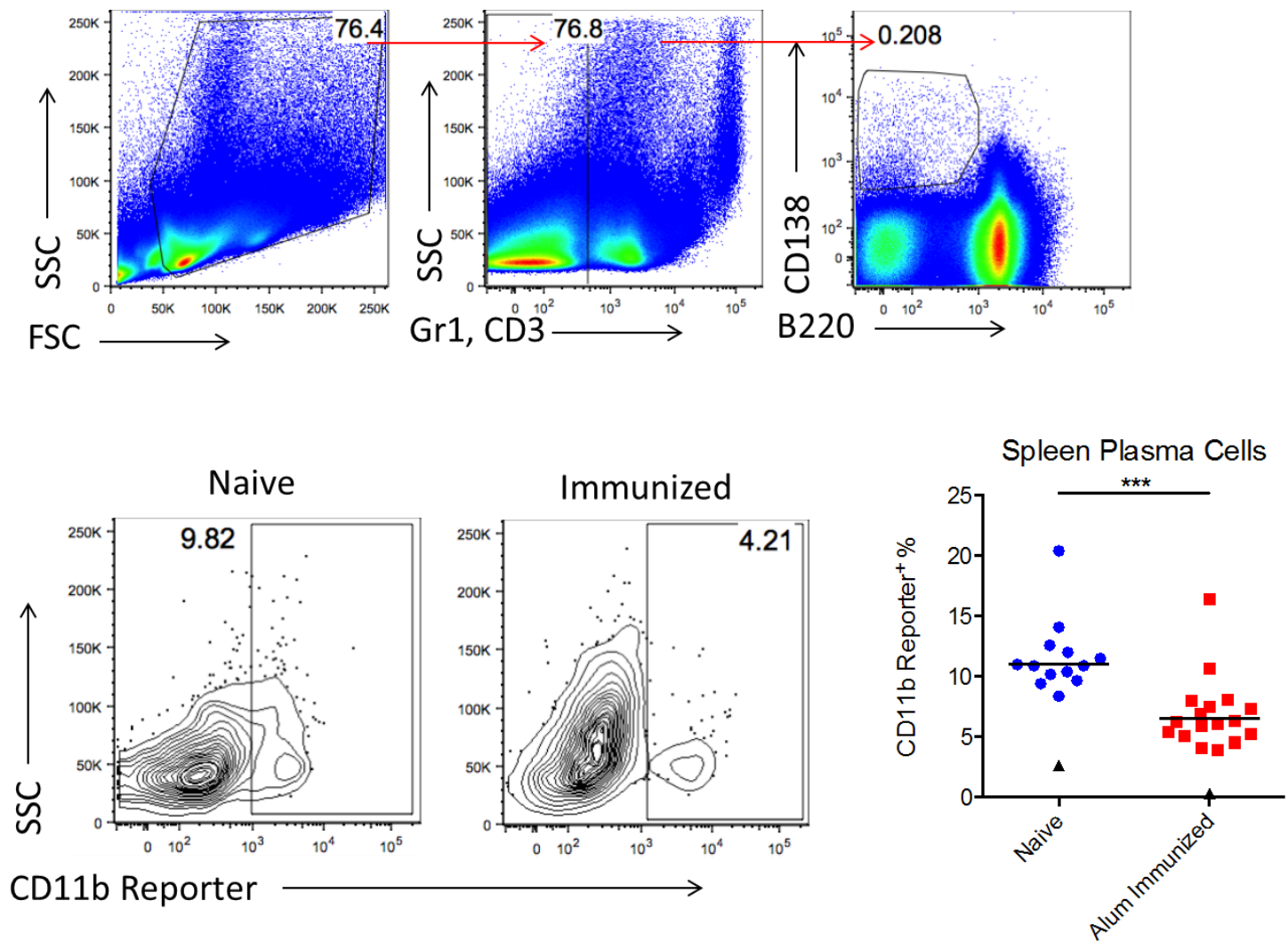

Figure 25: The impact of immunization on CD11b expression in splenic plasma

cells. FRT mice were immunized with $100 \mu \mathrm{g}$ of OVA suspended in Alum via i.p. injection. Spleens were harvested on day 14 post-immunization alongside naïve littermates. Gating strategy for identifying plasma cells (top) and representative dot-plots for $\mathrm{CD} 11 \mathrm{~b}$ expression (bottom) are shown. Reporter-negative littermates were used as referential controls, shown by $\boldsymbol{\Delta}$. Summarized data are from at least three independent experiments. $* * * \mathrm{P}<0.0001$ (unpaired two-tailed Student's t-test). 


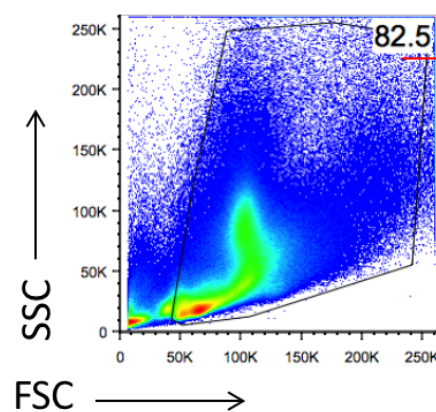

FSC

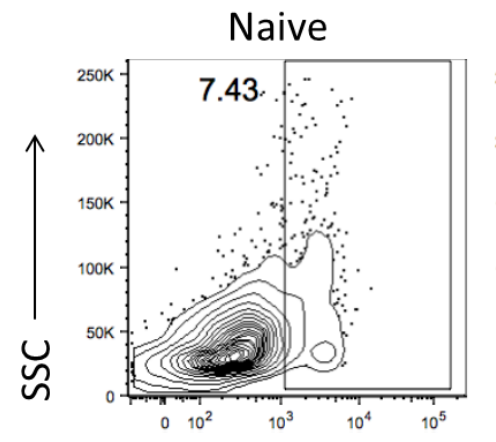

CD11b Reporter

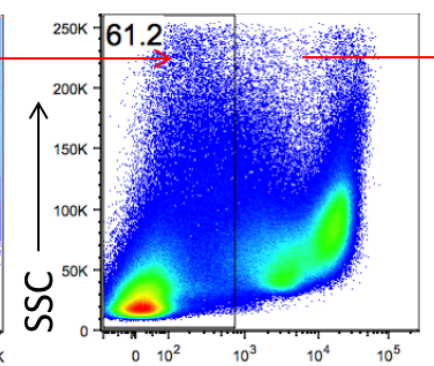

Gr1, CD3

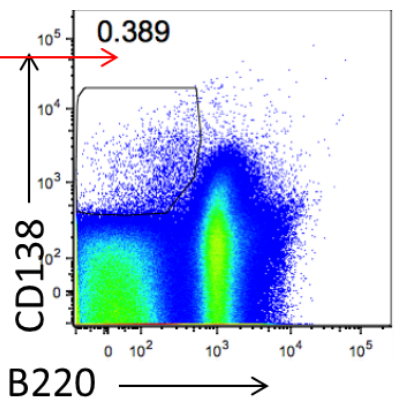

Bone Marrow Plasma Cells

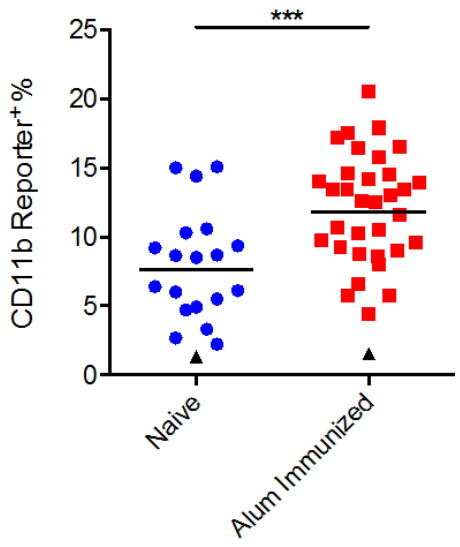

Figure 26: The impact of immunization on CD11b expression in long lived bone

marrow plasma cells. FRT mice were immunized with $100 \mu \mathrm{g}$ of OVA suspended in Alum via i.p. injection. Spleens were harvested on day 14 post-immunization alongside naïve littermates. Gating strategy for identifying plasma cells (top) and representative dot-plots for CD11b expression (bottom) are shown. Reporter-negative littermates were used as referential controls, shown by $\boldsymbol{\Lambda}$. Summarized data are from at least three independent experiments. $* * * \mathrm{P}<0.0001$ (unpaired two-tailed Student's t-test). 


\section{Progression of lupus-like disease in mice impacts B cell expression of CD11b}

Given the effectiveness of the FRT reporter system for examining B cell expression of CD11b in the immunization setting, we sought to utilize it in lupus-like models for increased disease-relevance. The Fas ${ }^{\mathrm{lpr}}$ mouse strain carries a loss of function mutation for the Fas gene, which is vital to Fas-FasL interaction and driving apoptosis of lymphocytes. This defect causes immense lymphoproliferation and lymphadenopathy, glomerulonephritis, and systemic autoimmunity, and is widely used as a murine model for SLE in both the MRL and B6 backgrounds $(195,196)$. We crossed this line with our FRT reporter strain to obtain FRT-Fas ${ }^{\text {lpr }}$ mice. Expression of CD11b on relevant B cell populations was observed from early life through disease onset at 3 months, which was confirmed by high titers of anti-dsDNA autoantibody in the serum (Figure 27).

The follicular and marginal zone B cells of the spleen showed very low expression of CD11b. This level was not affected with the progression of lupus-like disease (Figure 28). The splenic memory B cell CD11b+ population was much lower than that observed in healthy animals, and appeared to decrease as disease progressed. Though expression was initially very low in the GCs, they too revealed a decreased expression with age. By 4 months old, CD11b expression was nearly indistinguishable from the autofluorescence of reporter-negative controls.

The CD11b expressing fraction of splenic PCs was much higher than all other subsets, similar to observations in the healthy immunization model. At the earliest time point, an average of $4.5 \%$ expression can be seen which then steadily falls to nearly undetectable levels by month 4 (Figure 29). Long-lived bone marrow PCs conversely maintain CD11b expression over time, though with some volatility. 
The early developmental stages of B cells all display extremely low amounts of CD11b in this model; all below 1\% average expression (Figure 30). However, they do maintain a consistent pattern of expression with healthy animals; demonstrating notable CD11b expressing populations in the pre-pro and immature fractions while expression in the pro B and pre-B stages is minimal. Progression of disease appears to have no impact on these cells, with a slight increase in immature B cells being the exception.

As a whole, CD11b expression in B cells is much lower in the Fas ${ }^{\text {lpr }}$ disease state than in healthy animals. With the exception of plasma cells, all B cell subsets appear to have negligible $\mathrm{CD} 11 \mathrm{~b}$ expressing populations. Whether this is an effect of lupus-like disease, a unique bias caused by the Fas ${ }^{\mathrm{lpr}}$ model, or generalized inflammation remains to be determined. 


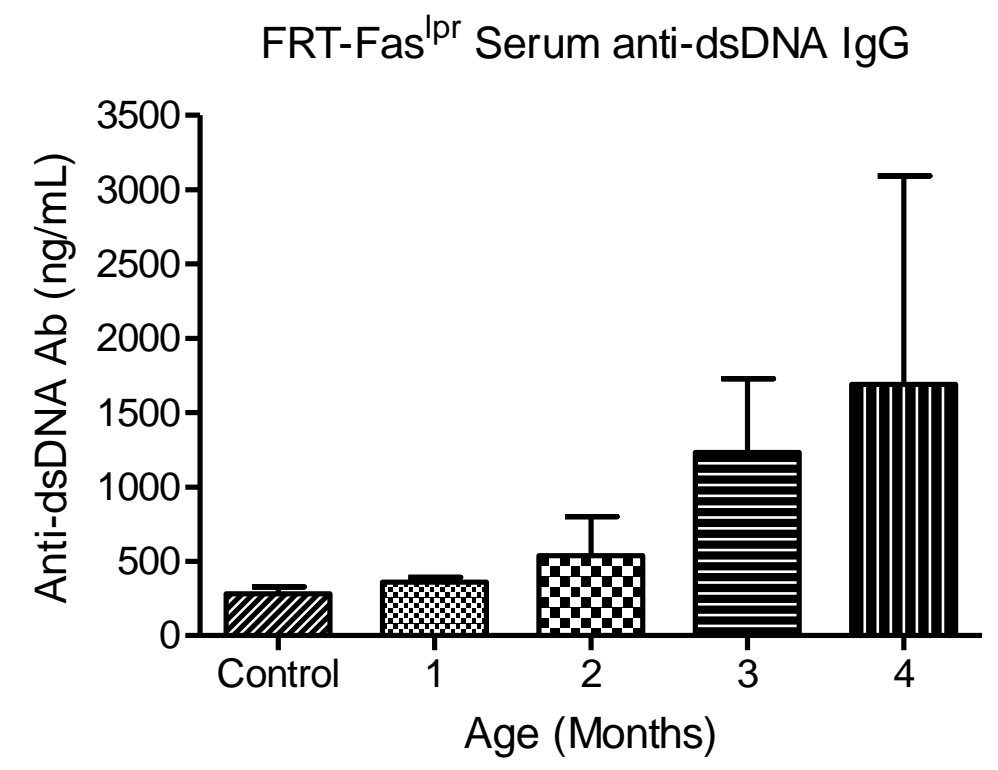

Figure 27: Progression of autoimmune disease in FRT-LPR mice. Fas ${ }^{\mathrm{lp} / \mathrm{pr}}$ were crossed with the CD11b-reporter FRT strain to obtain FRT-Fas ${ }^{\text {lpr/pr }}$ animals. Progression of autoimmune disease was confirmed by measurement of anti-dsDNA antibody at given time-points. 

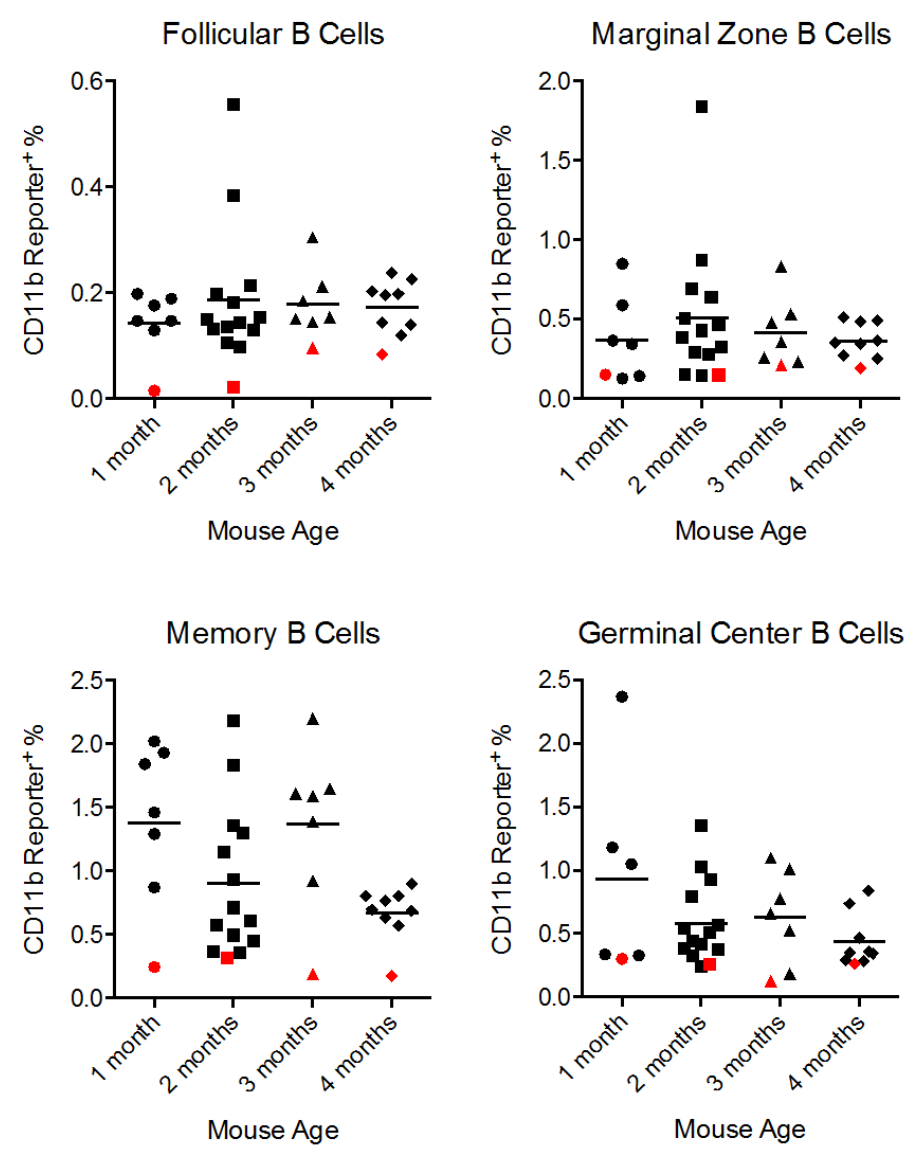

Figure 28: Fas $^{\text {lpr }}$ autoimmune disease impacts splenic B cell CD11b expression. FRT-

Fas $^{\mathrm{lpr}}$ animals were aged to 1 month, 2 months, 3 months, or 4 months before sacrifice.

Splenic populations of follicular, marginal zone, memory, and GC B cells were identified as previously shown above. Reporter-negative littermates were used as referential controls, shown in red. A minimum of $n=6$ were used for each time-point. 

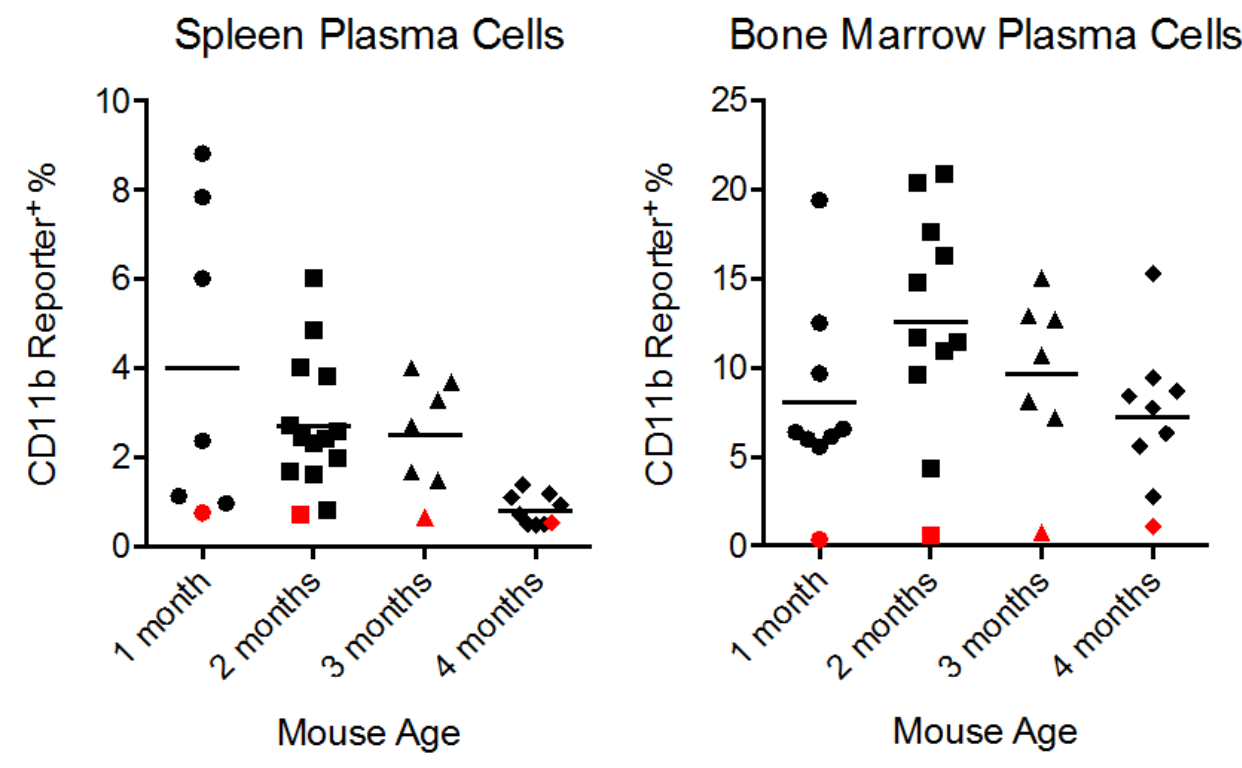

Figure 29: Fas $^{\mathrm{lpr}}$ autoimmune disease impacts plasma cell CD11b expression. FRTFas $^{\text {lpr }}$ animals were aged to 1 month, 2 months, 3 months, or 4 months before sacrifice. Splenic and bone marrow populations of PCs were identified as previously shown above. Reporter-negative littermates were used as referential controls, shown in red. A minimum of $n=6$ were used for each time-point. 

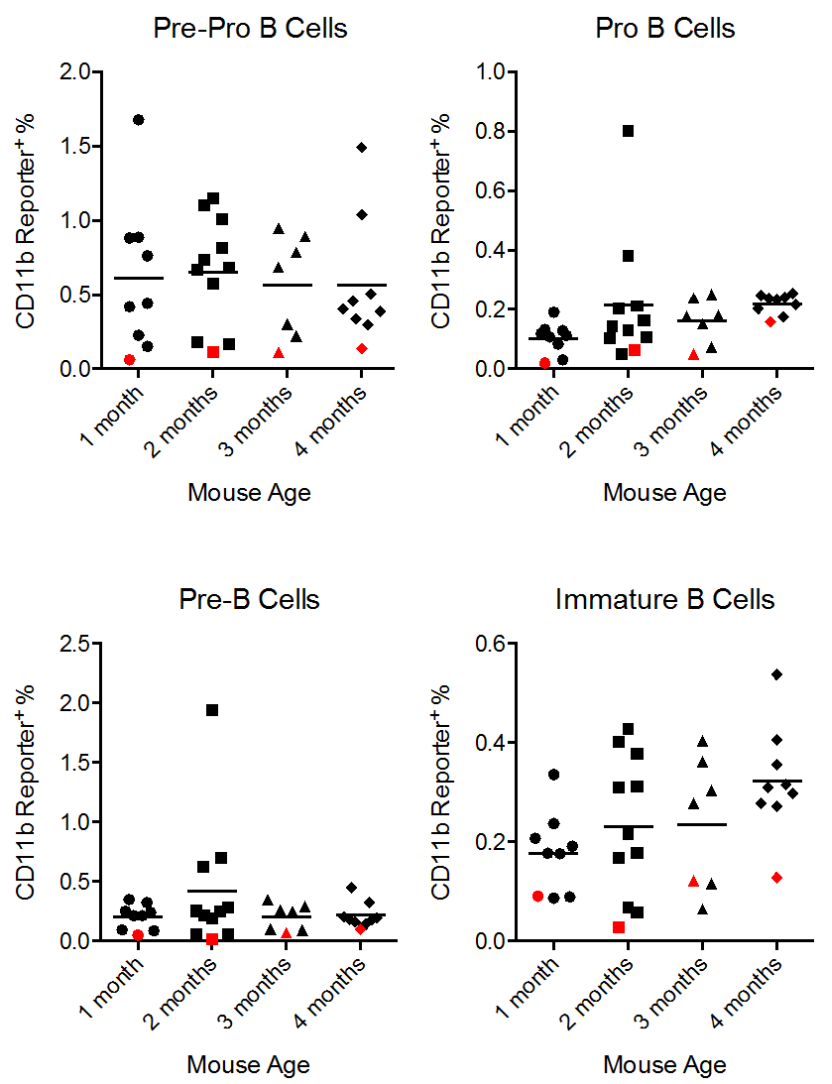

Figure 30: Fas $^{\text {Ipr }}$ autoimmune disease impacts developmental B cell CD11b

expression. FRT-Fas ${ }^{\mathrm{lpr}}$ animals were aged to 1 month, 2 months, 3 months, or 4 months before sacrifice. Bone marrow populations of developing B cells were identified as previously shown above. Reporter-negative littermates were used as referential controls, shown in red. A minimum of $n=6$ were used for each time-point. 


\section{Comparison of the CD11b-expressing plasma cell repertoire.}

Advances in high-throughput sequencing have allowed for large-scale analysis and immune profiling of the BCR repertoire in a given population. This technique has been used to study a number of autoimmune disorders, as well as assist in antibody discovery $(197,198)$. The established role for CD11b in downregulation of autoimmune activation suggests that its expression on B cells may correlate with a unique antigen specificity profile. Thus, we sought to utilize this technique to compare the BCR repertoires of the CD11b expressing and non-expressing PC subsets.

To examine this, we crossed our FRT reporter strain with the ABIN1 lupus-like model. A20-binding inhibitor of NF- $\mathrm{BB}$ (ABIN1) is a ubiquitin-binding protein that was identified as a SLE susceptibility loci in genome-wide association studies $(199,200)$. The $\mathrm{ABIN}^{\mathrm{D} 485 \mathrm{~N}}$ strain carries a non-functional mutant form of human ABIN1 (D485N) and has been used in several studies of B cell autoimmunity and as a mouse model of lupus (201-203). Additionally, this system is not subject to the bias of the Fas ${ }^{\text {lpr }}$ model whose lack of Fas-FasL interaction heavily skews selection of the B cell population. Progression of autoimmune disease in FRT-ABIN1 mice was confirmed via serum levels of antidsDNA IgG antibody (Figure 31). In a preliminary study, 5 month old ABIN1 animals displayed distinct CD11b expression in the PC population with subsets ranging from 3.7 to $25.7 \%$ (Figure 32). isolated the paired CD11b expressing and non-expressing subsets from individual animals for BCR repertoire sequencing and comparison. This is an ongoing study, and we expect analysis of these BCR repertoires to provide meaningful insight into the properties of the CD11b expressing plasma cell population. We will also 
determine if CD11b expression is protective against the autoimmunity or nephritis that occurs in ABIN1(D485N) mice. 
FRT-ABIN1 Serum anti-dsDNA IgG

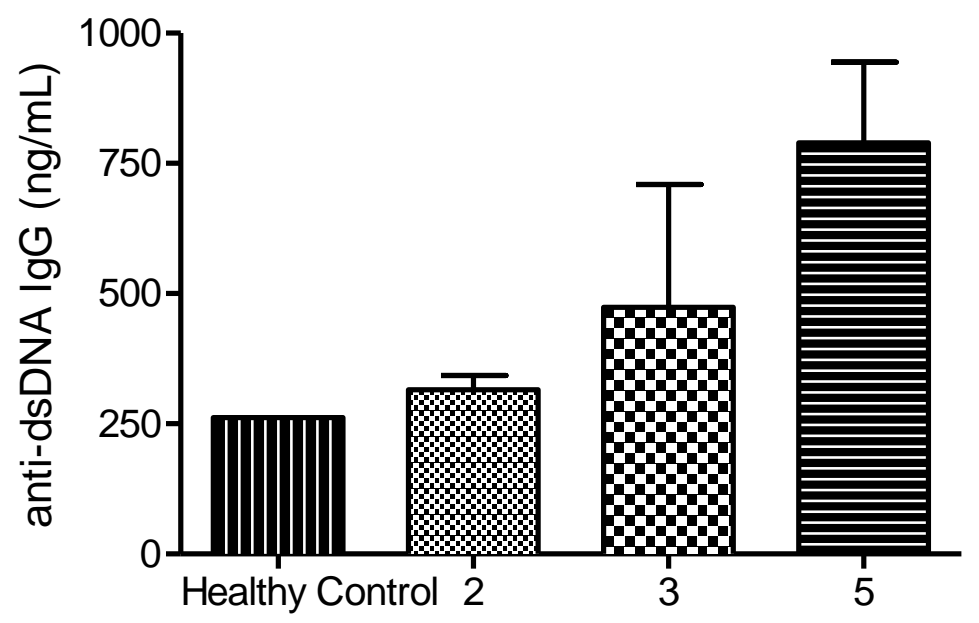

Figure 31: Progression of autoimmune disease in FRT-ABIN1 mice. $A B I N 1^{D 485 N}$ mice were crossed with the CD11b-reporter FRT strain to obtain FRT-ABIN1 animals. Progression of autoimmune disease was confirmed by measurement of anti-dsDNA IgG antibody at given time-points. 

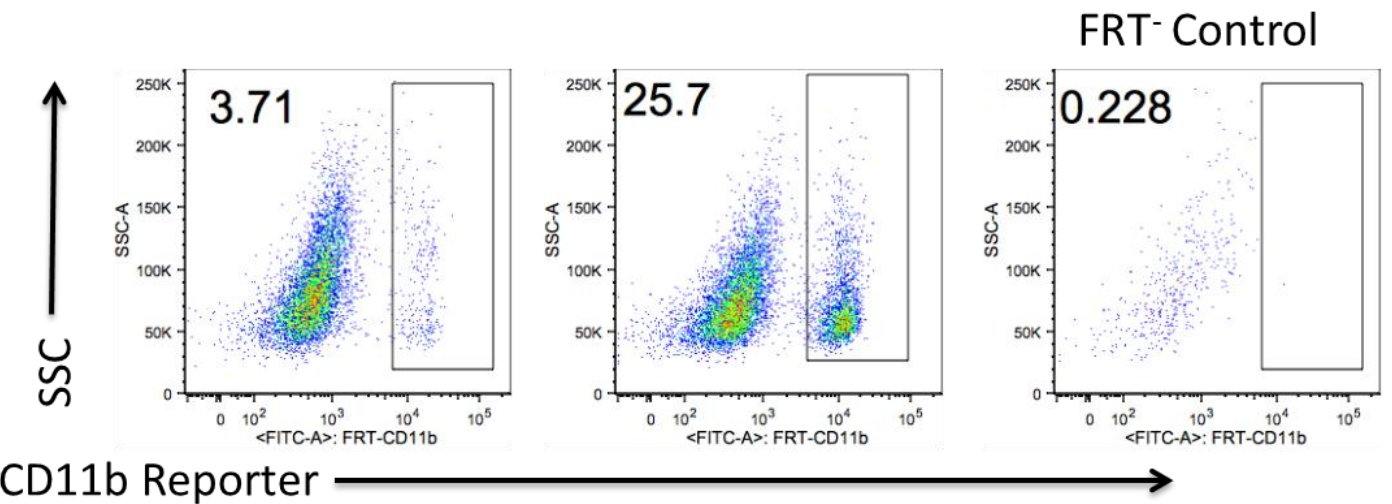

Figure 32: FRT-ABIN1 plasma cell CD11b expression. FRT-ABIN1 animals were aged to 5 months. The splenic population of PCs was identified as previously shown above. Gating for the CD11b expressing subset is shown, based reporter-negative control. 


\section{DISCUSSION}

The identification of $\mathrm{CD} 11 \mathrm{~b}$ as a regulator of BCR signaling revealed the potential for CD11b expression in multiple B cell subsets. As CD11b is conventionally associated with myeloid cells, its presence on B cell populations has been largely overlooked. We have demonstrated novel sub-populations of CD11b expressing B cells in multiple subsets, which are dynamically influenced by immune setting.

CD11b expressing B cell subsets have been previously identified, namely in the memory B cell, B1 B cell, and Peyer's patch resident plasma cell subsets $(169,189,193)$. Using a CD11b reporter, we were able to identify several more populations of $\mathrm{CD}_{11} \mathrm{~b}^{+} \mathrm{B}$ cells in immunization and autoimmune conditions. Early stages of B cell development in the bone marrow have extremely low CD11b expressing populations, suggesting CD11b does not impact B cell development in a significant way. Germinal center B cells of the spleen retained a relatively small population of CD11b expressing cells, which decreased even further after immunization. Our reporter model confirmed previous reports of a substantial CD11b expressing fraction of memory B cells. This expression increased following immunization, which is likely to assist in migration and tissue homing following generation as previously shown (169). Memory B cell CD11b expression was lower in autoimmune mice and decreased further with disease severity, which may be indicative of a regulatory role breakdown in addition to function in migration. Follicular and marginal zone B cells express CD11b at a comparatively lower level, which was significantly reduced after immunization. Indeed, in lupus-prone mice, CD11b expression was nearly absent in these cells, and did not change over the course of disease. 
In MRL-lpr lupus mice, autoreactive B cells are able to avoid follicular exclusion due to lack of Fas-FasL selection and become major producers of anti-dsDNA antibody (204). It is possible that populations of CD11b expressing B cells in which CD11b is actively serving as a negative regulator are being outcompeted by these autoreactive B cells unchecked by Fas-FasL deletion. Thus, the fraction of $\mathrm{CD} 11 \mathrm{~b}^{+} \mathrm{B}$ cells in these subsets is dwarfed by the $\mathrm{CD}_{11} \mathrm{~b}^{-}$, resulting in a much lower frequency in the total population.

Expanding upon the CD11b expressing plasma cells previously identified in the Peyer's patch, we observed CD11b positive populations of both splenic and bone marrow resident PCs. In the splenic population, which are typically short-lived PCs, CD11b expression decreased following immunization. The aforementioned CD11b expressing Peyer's patch PCs were found to proliferate and produce antibody at a higher rate than $\mathrm{CD}_{11} \mathrm{~b}^{-}$cells. Increased CD11b expression may serve as a negative regulator to better control these hyper-responsive cells. Splenic PC CD11b could function in a similar role by tempering the response of newly formed PCs, which carry reduced expression as they complete differentiation and exit the GC (Figure 33). Conversely, long-lived PCs of the bone marrow increased CD11b expression following treatment. Long-lived PCs do not activate and respond to $\mathrm{BCR}$ binding antigen or immune complex, but rather exist to provide persistent antibody after initial encounter with antigen $(114,205)$. Here, high CD11b expression may serve as an additional layer of regulation to prevent BCR signal activation of these cells and maintain a steady state of antibody production. In Fas ${ }^{\text {pr }}$ mice, both bone marrow and splenic PCs lost CD11b expression over time. Like the memory B cells, this is likely related to increased severity of disease as freshly formed 
PCs of the spleen lose regulatory elements and the migratory pattern of long-lived PCs is altered by a chronic state of immune response.

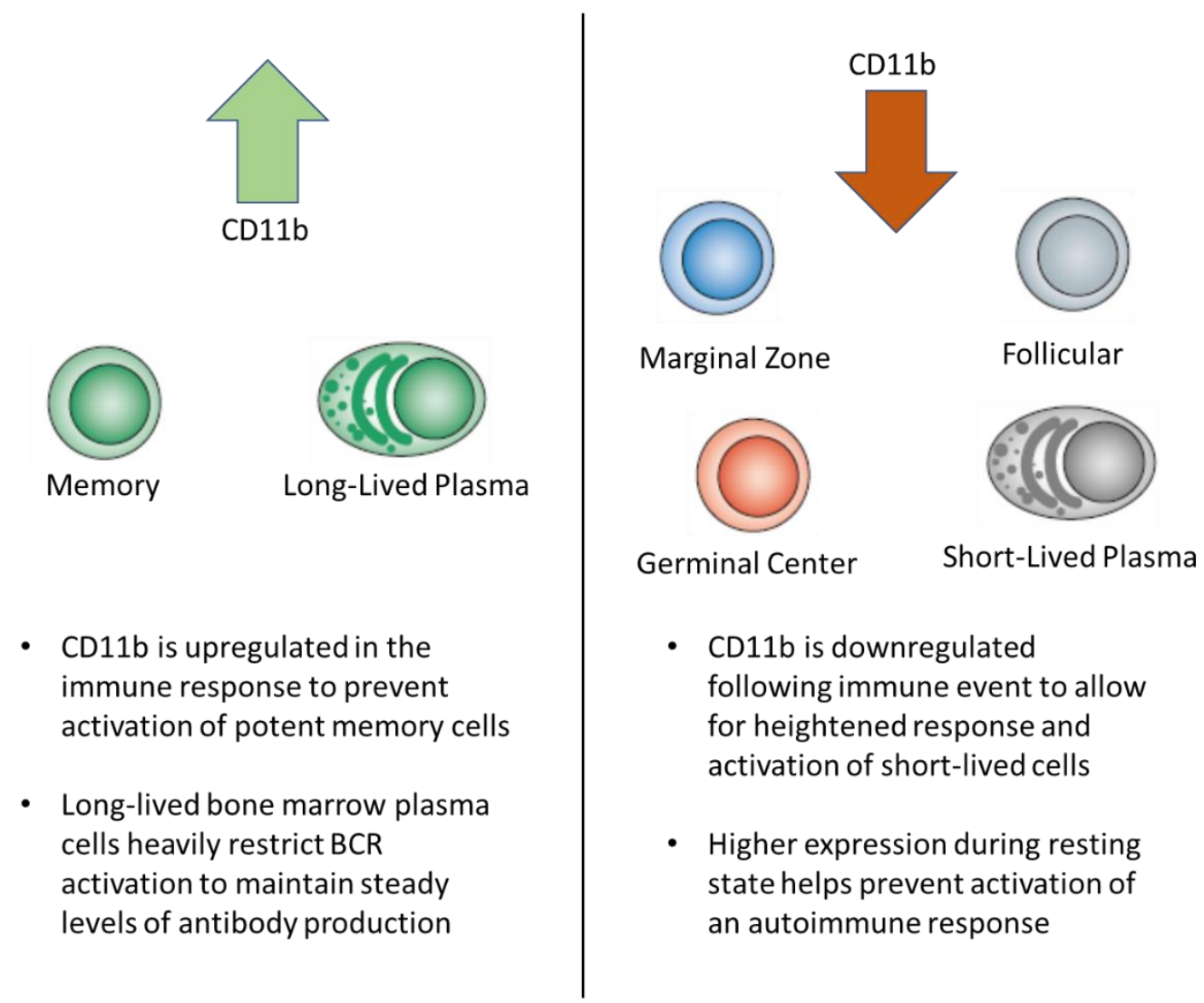

Figure 33: CD11b is dynamically expressed across different $B$ cell subsets

The variability in CD11b expression patterns seen across these B cell populations demonstrates its role as a dynamic regulator that serves the need of different functional niches. In splenic memory B cells and long-lived plasma cells of the bone marrow, increased expression of $\mathrm{CD} 11 \mathrm{~b}$ is following immunization may providing increased protection against unwanted activation. These B cell types are potent mediators of secondary responses, and increased CD11b downregulation of BCR signaling can prevent a pathogenic hyper-production of antibody. Conversely, the marginal zone, follicular, 
germinal center, and short-lived splenic plasma cells all reduced CD11b expression in the Th2 response. These are the formative and transient mediators of humoral immunity, acting to generate a response and persist only long enough to permit clearance of pathogen before dying off. Decreased expression of negative regulator CD11b during the crucial stages of the humoral immune reaction can allow for more effective engagement of the BCR, enhancing B cell differentiation and subsequent antibody response. Comparatively increased CD11b expression during the naïve state could be acting as a prevention mechanism of acute autoimmune activation, restricting BCR engagement until a true systemic immune reaction takes place.

Examination of $\mathrm{CD} 11 \mathrm{~b}$ in the disease state will require more thorough studies. The B cell selection bias of the Fas ${ }^{\mathrm{lpr}}$ model alters the B cell population and causes unintended skewing of the $\mathrm{CD} 11 \mathrm{~b}$ expressing fractions. Other models of lupus which do not have a direct effect on $\mathrm{B}$ cells, such as $\mathrm{ABIN} 1^{\mathrm{D} 485 \mathrm{~N}}$, may reveal more about $\mathrm{CD} 11 \mathrm{~b}$ in disease progression.

Preliminary experiments (data not shown) have demonstrated that CD11b expression or non-expression on plasma cells and mature B cells is conserved in vitro for up to 72 hours in stimulation conditions. This suggests that CD11b expression is not a transient property, is rather a long-term characteristic of these populations. Whether expression of this regulator is a germline-intrinsic property or something that can be induced remains to be seen. Additionally, the functional response of the $\mathrm{CD} 11 \mathrm{~b}^{+}$and CD11b- populations of these subsets must be evaluated. Ongoing experiments comparing the $\mathrm{BCR}$ repertoire of the divergent $\mathrm{CD} 11 \mathrm{~b}$ subsets will reveal much about the functional impact of CD11b. It is anticipated that the $\mathrm{CD} 11 \mathrm{~b}^{+}$will contain a higher frequency of 
autoreactive BCR specificities, which would indicate CD11b expression is controlled by B cell negative selection; but this remains to be been.

The identification of dynamic CD11b expression on novel B cell subsets provides the potential outline of a complex regulation mechanism for BCR signaling. Further experiments are necessary to understand the function and precise role of $\mathrm{CD} 11 \mathrm{~b}$ in these varying stages of B cell development. 


\section{CHAPTER III}

THE IMPACT OF CD11B POLYMORPHISM ON B CELLS IN SYSTEMIC LUPUS ERYTHEMATOSUS 


\section{INTRODUCTION}

Though the intended function of the immune system is to protect from foreign pathogens, genetic abnormalities and environmental factors can cause malfunction leading to pathological effects and damage to self, categorized as autoimmune disease. Systemic lupus erythematosus (SLE) is an autoimmune disorder characterized by the degradation of B cell tolerance, leading to the production of antibodies against selfantigens which cause severe inflammation in the joints and kidneys (206). Many different single nucleotide polymorphisms (SNPs) have been shown to contribute to SLE pathogenesis. One such SNP, rs1143679, causes a mutation in CD11b $(207,208)$. Herein, we will overview SLE as a disease and review the known impacts of CD11b mutation on disease outcome.

\section{Presentation and Causes of SLE}

Modern study of SLE has revealed several different forms, presentations, and causes of the disease; however, a set of characteristic hallmark symptoms is shared among them. Cutaneous manifestations are nearly universal in SLE, appearing in over $75 \%$ of patients, which can then be partitioned into lupus erythematosus-specific or nonspecific symptoms (209). The disease's namesake results from an acute cutaneous lupus erythematosus (ACLE) that often occurs as a butterfly rash on the face, or as a more generalized maculopapular exanthema (210). Another SLE subset, discoid lupus erythematosus (DLE) is characterized by inflammatory plaques, follicular hyperkeratosis, and scarring leading to to recognizable patterns of hair loss (211). Over 90\% of SLE patients also have musculoskeletal system involvement. Myalgia, arthralgia, and arthritis are the most common symptoms of this type (212). The central nervous system is affected in roughly $30 \%$ of SLE cases. However, the symptoms are very nonspecific, and 
not reliable identifiers of the disease (213). Psychosis, depression, epileptic seizures, and transverse myelitis are among the peripheral neuropathies seen in SLE (214).

Though only $50 \%$ of SLE patients develop lupus nephritis (LN), it is one of the major causes of morbidity and hospitalization among all SLE manifestations (215). Because of the aggressive nature of renal involvement, the threshold for preforming a biopsy is somewhat low (216). Proteinuria and hematuria are standard signs of LN. Glomerular nephritis followed by subsequent chronic renal damage and kidney failure remain the classic pathological symptoms of LN.

The genetic component to SLE has long been established, as first seen in the heightened rate of the disease in monozygotic twins. Genes involving the major histocompatibility complex (MHC) were the first major loci found to associate with SLE. Many of the SNPs in this region remain among the most impactful on susceptibility (217). Genome-wide association studies have revealed a number of additional genes related to SLE and other immune disorders. They typically fall into one of three major mechanisms: Interferon (IFN) signaling, clearance of immune complexes and cellular waste, and lymphocyte signaling and activation (218). Some major lymphocyte activation gene loci involved in autoimmune disorders include PTPN22, IL-10, IL-21 A, CD44, LYN, and IKZF2. A few IFN pathway loci of interest are PRDM1, IRF5, IRF7, and SOCS1. Lastly, relevant clearance genes comprise FCGR2A, FCGR2B, and CLEC16A (219). While these loci are ubiquitous to several immune disorders including SLE, many monogenic causes of SLE have been identified. Some of the more notable genes include C2, C3, DNASE1, FAS/FASL, and ITGAM (220-224). 


\section{Pathogenesis of SLE}

There are several different cell types and subsets implicated in the pathogenesis of SLE, and B cells are most predominately associated with the disease due to the large role of autoreactive antibodies, notably ANAs. These ANAs are the result of a break in tolerance of self-reactive B cells. The generation of hallmark ANAs in SLE first requires a breakage of B cells from one or more of tolerance pathways. Indeed, disfunction within the B cell itself has been associated with SLE more than other cell types. Risk-loci analyses revealed B cell genes mostly strongly correlated with SLE while alleles from memory T cells were implicated in rheumatoid arthritis, further cementing SLE as a B cell-centric disease (225). Many of these B cell defects disrupt the aforementioned tolerance mechanisms, leading to increased production of antibody regardless of antigen specificity. Combining this with the large fraction of immature B cells that are reactive to nuclear antigens creates the strong potential for the production of ANAs (226). Additionally, B cells may react to nuclear antigen without requiring BCR specificity through innate immune pathways (17). Defects in the B cell activation pathway may also contribute to the development of SLE, regardless of tolerance-induction. These mechanisms serve to regulate the strength and longevity of activation signals generated by BCR ligation, and their disruption can drive SLE (227).

High-affinity effector IgG and chronic antibody responses in SLE result from plasma cells and long-lived memory B cells differentiated from the germinal center. In several lupus mouse models, enhancement of the GC reaction is a hallmark phenomenon (228). Human SLE patients display increased frequency of peripheral pre-GC B cells, Tfh cells, and memory B cells which are indicative of the GC reaction (229). These abnormal 
GCs give rise to PCs and the production of pathogenic $\operatorname{IgG}$ antibodies through multiple apparatuses. Firstly, the entry of autoreactive naïve B cells into the GC can contribute to SLE. These cells are normally excluded from entering the B cell follicle and subsequent $\mathrm{GC}$ at a tolerogenic checkpoint that is not well understood $(230,231)$. Autoreactive B cells from SLE patients have been found to circumvent this mechanism entirely and proceed to the GC stage while non-SLE patient cells do not (232). Even if this tolerance checkpoint remains effective, non-autoreactive B cells may become self-reactive in the GC. The majority of pathogenic antibodies in SLE have undergone SHM. The large fraction of post-GC IgG autoreactive cells compared to the smaller amount on pre-GC autoreactive B cells suggests the autoreactive epitope is generated during the GC reaction. Autoreactive memory B cells have been shown to derive from this pathway, but the generation of autoreactive PCs via this mechanism is unproven (233-235).

In SLE patients, the GC has increased survival that enables them to generate higher amounts of autoreactive PCs. Critical GC cytokine BAFF is markedly elevated in SLE patients (236). Increased amounts of BAFF not only encourages the survival of GC B cells that may otherwise fail selection checkpoints, but also promotes Tfh expansion. Activity of the Tfh cells is able to promote autoreactive GC B cells, and increased numbers of Tfh cells has been linked to SLE. Tfh cells produce IL-21, which has been shown to be critical in the development of self-reactive PCs and is increased in SLE patients $(237,238)$. OX40L expression on B cells is essential for Tfh maturation, and overexpression of B cell OX40L has been suggested to enhance the autoimmune response via Tfh expansion in some lupus mouse models (239). Mutations in OX40L have also 
been associated with human SLE, further demonstrating a role for Tfh cells in the disease (240).

\section{CD11b Mutation rs1143679 and SLE}

A coding variant in ITGAM has been associated with incidence of SLE. Identified as rs1143679, this mutation results in an arginine to histidine amino acid substitution at the $77^{\text {th }}$ position, described as $\mathrm{R} 77 \mathrm{H}(119)$. This substitution is in the extracellular domain of the molecule, very near the primary ligand-binding portion. The change of a larger and more highly charged amino acid to a smaller, less drastically charged one near a crucial site has implications on the molecule's function $(124,207)$.

Studies have identified differences frequency of the rs1143679 variant across multiple populations. While other ITGAM variants exist, rs1143679 was found to be the most strongly associated with SLE across different groups. In one study, $17.2 \%$ of SLE patients of European ancestry possessed this variant, while only $10.4 \%$ of healthy subjects were carriers. For patients of African descent, frequency was slightly lower with $15 \%$ incidence for the SLE group and $10 \%$ in healthy controls. Interestingly, analysis of the Gullah subpopulation of African descent found higher association with SLE at $20 \%$ disease incidence and $11 \%$ in controls (124). In a different study, Hong Kong Chinese and Thai populations were observed, finding only $1.1 \%$ and $6.2 \%$ SLE association, respectively. Healthy patient mutation frequency was expectedly lower at $0.51 \%$ and $2.1 \%$ (241). An examination of Hispanic-Americans revealed a 17\% SLE association and $9 \%$ in controls (242). For a Northern Indian cohort, incidence was $14.3 \%$ for SLE patients and $8.7 \%$ in healthy individuals (243). These data demonstrate a varying range of 
rs1143679 frequency among varied populations, with significant differences even in small sub-groups.

The effects of this ITGAM variant have been examined in multiple cell types. Notably, total expression of CD11b is not altered in variant-carrying monocytes and neutrophils. Even CD11b activation via PMA did not affect expression, indicating the mutation does not alter inside-out signaling or functional expression. However, phagocytosis in these cells is impaired. Both ex-vivo variant macrophages and varianttransfected macrophage cell lines demonstrate reduced phagocytosis via iC $3 \mathrm{~b}$. Adherence assay revealed reduced binding to iC3b, ICAM1, DC-SIGN, and Fibrinogen in variant cells. Binding of CR3 inhibits release of TLR7/8 cytokine in healthy cells, but inhibition was lost in variant monocytes (207). This reveals a potential mechanism for rs1143679 in SLE pathogenesis via dysfunctional CD11b regulation of TLR7/8 activation in response to inert nuclear antigen (244). Supporting this, CD11b has also been shown to mediate C3 activation of immunosuppression and tolerance in response to UV light damage, an established risk factor of SLE (245). Inflammatory cytokine production is normally inhibited by CD11b. Indeed, variant-expressing macrophage lines have much higher levels of IL-6 than control cells following stimulation, demonstrating yet another deficiency in proinflammatory cytokine regulation (246).

The rs1143679 variant alters neutrophil function via Mac-1 disruption. In one study, primed variant-carrying neutrophils are shown to be deficient in phagocytosis of Mac-1 dependent opsonized particles, while several other phagocytosis receptor pathways are unaffected. Affected pathways included phagocytosis via the Fc receptor, which are less able to phagocytose IgG-coated particles than non-variant neutrophils. The 
same report also demonstrates reduced firm adhesion by variant neutrophils (247). Variant deficiency in adhesion has been examined in-depth, revealing a non-binding regulatory role of the $\mathrm{R} 77$ region of $\mathrm{CD} 11 \mathrm{~b}$. Under tensile force conditions, the $\mathrm{R} 77$ contributes to brief-catch bond stability of the binding region, allowing disassociation of the cytoplasmic tail domain of the alpha subunit from the beta subunit in binding conditions. The $\mathrm{R} 77 \mathrm{H}$ variant causes accelerated formation of these catch-bonds, leading to non-disassociation of the cytoplasmic domains and prolonging the Mac-1 complexligand binding lifetime (248). The complexities of these structural interactions are still largely unknown and must be investigated further to better understand how this variant leads to disease susceptibility at a systemic level.

As stated above, total expression of CD11b was altered by the rs 1143679 variant expressed in cell lines. However, Maiti et al. have shown a dose-dependent change in CD11b expression in SLE patients depending on risk allele transcripts. Patients homozygous for the variant had reduced CD11b expression on peripheral blood mononuclear cells (PBMCs) at both the surface and RNA expression levels compared to heterozygous patients. Within the heterozygous cohort, they also identified differences in expression and regulation of the variant versus non-variant forms of CD11b. Strikingly, expression of the non-variant form was seven times higher than the variant. This was found to be an effect of a damaged transcriptional enhancer region in the rs1143679 variant allele (249). These findings demonstrate the crucial protective effect of a single copy of the non-variant form of CD11b and suggests a reason for the common prevalence of the variant in healthy patients despite the strong association with SLE. However, a recent report by Roberts et al. directly contradicts these data, suggesting that monocyte 
and NK cell expression of CD11b is not altered by rs1143679 in any way. Their results found no difference in CD11b expression between homozygous, heterozygous, and nonvariant genotype groups in classical monocytes, non-classical monocytes, CD16 expressing monocytes, and four NK subsets (250). These opposing publications exemplify the current lack of understanding of the role of rs1143679 and the necessity for further investigation of this variant form of CD11b.

Association studies have found a number of disease trends in rs1143679 carrying SLE patients. This ITGAM variant, as well as others, are associated with elevated IFN-I activity in SLE. Cells from these subjects also had increased expression of IFNB and IRF7 transcripts, suggesting a potential pathway for type-1 IFN production (251). Other proinflammatory cytokines IL-6, TNF- $\alpha$, and IL- $1 \beta$ are higher in SLE patients with this variant as well $(207,252)$. The same group of ITGAM variants has also been associated with anti-dsDNA autoantibody (253). In some SLE populations, the R77H variant is linked to higher levels of antiphospholipid antibody (254). Though this variant has been associated with SLE in multiple studies, more recent data has found that this and other ITGAM risk alleles are much more strongly associated in patients with renal nephritis than those without. The same trend has been shown in SLE patients with neurological disorders (241). This association of rs1143679 with more severe disease phenotype further supports the function of $\mathrm{CD} 11 \mathrm{~b}$ as a key regulator.

Due to the prevalence of rs1143679, the Mac-1 receptor/CD11b has become a potential therapeutic target for treatment of SLE. In one study, CD11b agonist leukadherin-1 (LA1) was able to reduce kidney neutrophil influx and proteinuria in an inducible nephritis mouse model (255). The same treatment can also reduce production of 
proinflammatory cytokines IL-1 $\beta$ and IL-6 in neutrophil and macrophages (251). LA1 has been tested in the MRL/lpr lupus model as well. Here, administration of the small molecule resulted in reduced IFN-I responses, partial integrin activation, and protection from organ injury (251). The emerging use of CD11b agonist as a therapeutic shows promise, but the expression of ITGAM across multiple cell types exposes a number of potential off-target effects in humans. A better understanding of CD11b function in individual cell types is necessary to develop more highly specific treatments able to target the pathogenic involvement of this pathway in SLE.

Though B cells play a central role in SLE, the relationship of B cell function and mutation in ITGAM is poorly defined. Our previous work has revealed CD11b as a novel regulator of BCR signaling and B cell activation, suggesting the presence of a B cellintrinsic defect caused by ITGAM mutation in rs 1143679 expressing SLE patients. Indeed, a human B cell line expressing this mutant form was found to have defects in negative regulation of the BCR signaling pathway (136). However, the functional impact of this mutation in primary B cells from SLE patients has not been examined. In the following study, we examine the prevalence of rs1143679 in nephritic SLE patients in the Louisville region and attempt to correlate its incidence to clinical outcome. We also compare the function of B cells from ITGAM variant and normal patients in vitro. 


\section{MATERIALS AND METHODS}

Immunizations and Ab Detection

Human and anti-dsDNA antibody was detected using respective anti-dsDNA ELISA kits (Signosis Inc). Antinuclear antibody (ANA) was detected using HEp-2-coated antigen substrate slides and confocal microscopy (MBL International).

Antibodies, Flow Cytometry, and Cell Sorting

Single-cell suspensions of PBMCs were treated with Fc-blocker for 10 minutes on ice followed by staining with appropriate fluorochrome-labeled antibody or isotype controls for at least 20 minutes at $4^{\circ} \mathrm{C}$. Cells were then washed with 10 volumes of PBS before resuspension in PBS for acquisition or Running Buffer (Miltenyi Biotech) for sorting. Flow cytometry samples were acquired using a FACSCanto cytometer (BD Biosciences) and analyzed using FlowJo software (Tree Star). Sorting was performed using FACSAria

III (BD Biosciences). Cell purity was confirmed at greater than $90 \%$ via flow cytometry. The following anti-human antibodies were used: fixable viability dye (eBioscience), human Fc-blocker, CD10, CD19, CD24, CD27, CD38, IgD (Biolegend).

\section{Human Subject Samples}

SLE patient blood was obtained from the University of Louisville Nephrology Department in collaboration with Dr. Dawn Caster and Dr. Kenneth McLeish. The study was approved by the Institution Ethical Board and blood samples were collected upon a written informed consent. Patient SLE classification varied on a case-by-case basis, as did time since first diagnosis. Blood samples were obtained immediately following draw 
for processing. Whole blood cells were frozen in $-80^{\circ} \mathrm{C}$ for DNA isolation. Plasma was obtained via centrifugation and frozen in aliquots at $-80^{\circ} \mathrm{C}$. PBMCs and Neutrophils were purified from remaining blood with a dual-histopaque density gradient. PBMCs were gradually frozen in freezing buffer $(90 \% \mathrm{FBS}, 10 \% \mathrm{DMSO})$ at $-140^{\circ} \mathrm{C}$. Neutrophils were frozen in TRIzol at $-80^{\circ} \mathrm{C}$ for RNA extraction.

Genotyping of ITGAM R77H rs1143679

DNA was extracted from $100 \mu \mathrm{L}$ of whole blood using a Blood \& Cell Culture DNA Maxi Kit (Qiagen). The presence of rs1143679 was identified via PCR and carried out with the following primers: 5'-CTGGTTTTTGTGTCATTCTTAGG-3'; 5'GAATCCCAGTCCCAGCCCG-3'. PCR products were then subjected to enzymatic digestion with endonuclease SsiL(C/CGC) (Thermo Fisher Scientific) before electrophoresis on a 2\% agarose gel. The ITGAM 77R allele was cleaved into 304, 24, and 22 base pair fragments. The $77 \mathrm{H}$ allele was cleaved into 328 and 22 base pair fragments.

\section{Hunan Plasmablast Culture}

Frozen whole PBMCs were thawed and counted. Cells were labeled with Carboxyfluorescein succinimidyl ester (CFSE) and washed. Whole-labeled PBMCs were cultured in a 96 well plate at $2.5 \times 10^{6}$ cells $/ \mathrm{mL}$ in the presence of $10 \mu \mathrm{g} / \mathrm{mL}$ anti-IgM, 0.33nM CpG, and 20ng/mL hIL-21 for 7 days. Upon well harvest, cells were used for flow cytometry and supernatant was saved in $-30^{\circ} \mathrm{C}$ for ELISA. 
Statistical Analysis

All quantitative data are shown as mean \pm s.e.m unless otherwise indicated. All samples were compared using two-tailed, unpaired Student's $t$ test. A $p$ value less than 0.05 was considered significant. Statistical analysis was performed with Prism (Graphpad Software). 


\section{A phenotypic survey of ITGAM mutation rs1143679 in SLE patients of the \\ Louisville region.}

The ITGAM mutation rs1143679 has been observed in multiple populations and ethnicities in many different countries, as previously discussed. We sought to examine this polymorphism in SLE patients within the Louisville, Kentucky region of the United States. Peripheral blood was collected from consenting SLE patient donors in care of the University of Louisville's nephrology clinic at varying ranges of age, disease state, treatment regimen, and time since diagnosis. Clinical diagnostic data was also provided.

We first performed genotyping for the R77H allele using whole blood. From a cohort of 38 patients, we identified 11 (28.95\%) patients homozygous for rs1143679 (described as HH) while $18(47.37 \%)$ and $8(21.05 \%)$ were heterozygous $(\mathrm{RH})$ or noncarriers (RR), respectively (Figure 34). A single patient carried an unknown SNP, identified by irregular PCR results. The incidence of the homozygous mutation is slightly higher than that of all other previously reported groups, though this may be due to variation in the low (relatively) sample size. Expectedly, the majority of patients (97.4\%) were female, thus no sex correlation could be investigated. Roughly $75 \%$ of patients were African American and 25\% were Caucasian, both of which had the same incidence of homozygous R77H (Figure 35). Surprisingly, and contrary to previously reports, we found no correlation in severity of lupus nephritis with homozygous rs1143679 mutation $(254,256,257)$. There was a slight, but non-significant, increase in the age of onset for carriers, averaging at 6.5 years. The frequency of joint and skin involvement was consistent among the groups, a roughly $82 \%$ and $62 \%$, respectively. Serum levels of C3 and C3 were also unaffected by ITGAM mutation. Level of anti-dsDNA IgG antibody 
did appear to increase with rs1143679 incidence, though this association was not statistically significant. Interestingly, there was a trending increase in serum creatine levels in homozygous and heterozygous carriers, but not in protein/creatine ratio.

We next preformed a panel staining on patient PBMCs to examine the effect of ITGAM mutation on the frequency of several B cell subsets. Our panel identified the transitional 1, transitional 2, mature naïve, plasmablast, non-switched memory, and double-negative memory B cell populations (Figure 36). Surprisingly, we did not observe any significant correlation in these populations with incidence of rs1143679 (Figure 37).

To examine the functionality of the B cells from these three groups, we cultured PBMCs in vitro in the presence of BCR, TLR9, and IL-21r stimulation to induce plasmablast differentiation. Among the three genotype cohorts, we observed no significant alteration the ability of these B cells to differentiate (Figure 38). We also found no impact of R77H mutation on the amount of ANA and anti-dsDNA antibody produced.

These summary data demonstrate no strong correlation of clinical outcome with homozygous or heterozygous ITGAM mutation. Combined with the lack of functional alteration in our in vitro assay, it appears rs1143679 does not cause a significant impact on B cell function or disease within our cohort. 


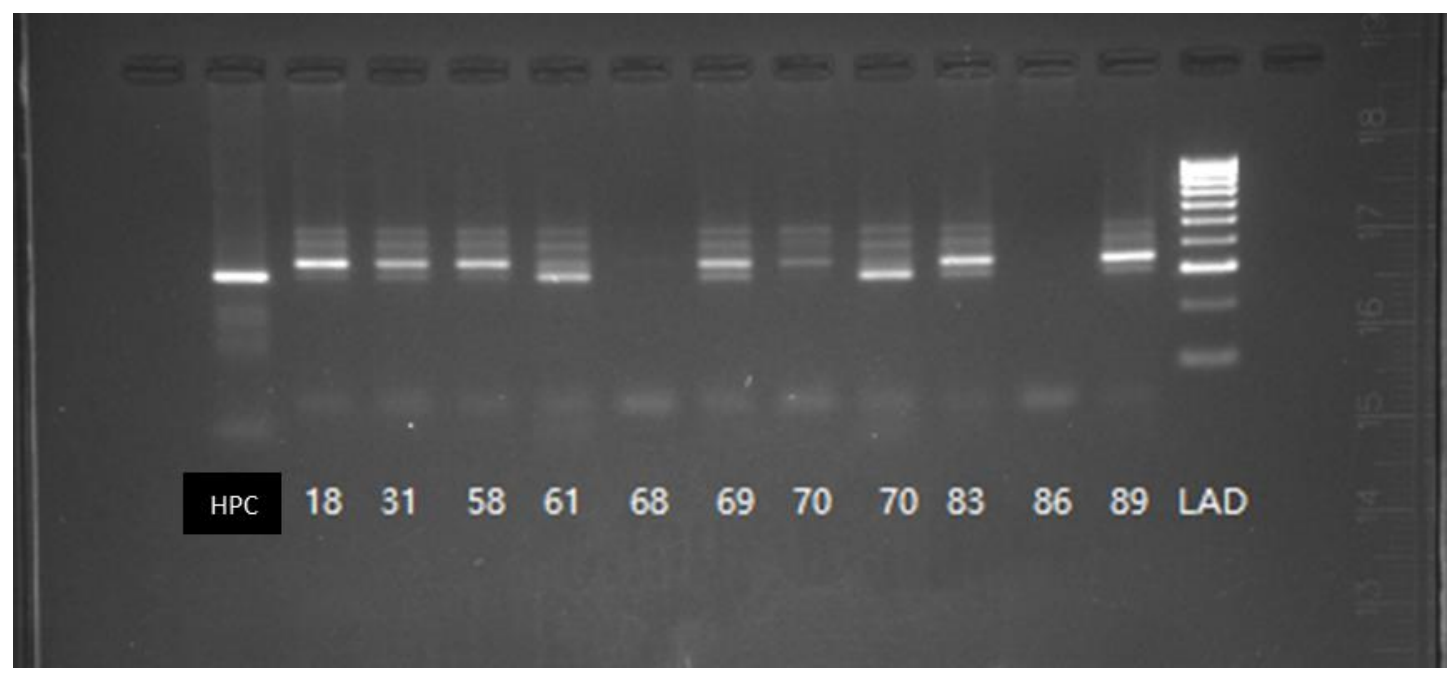

\section{R77H Genotype}

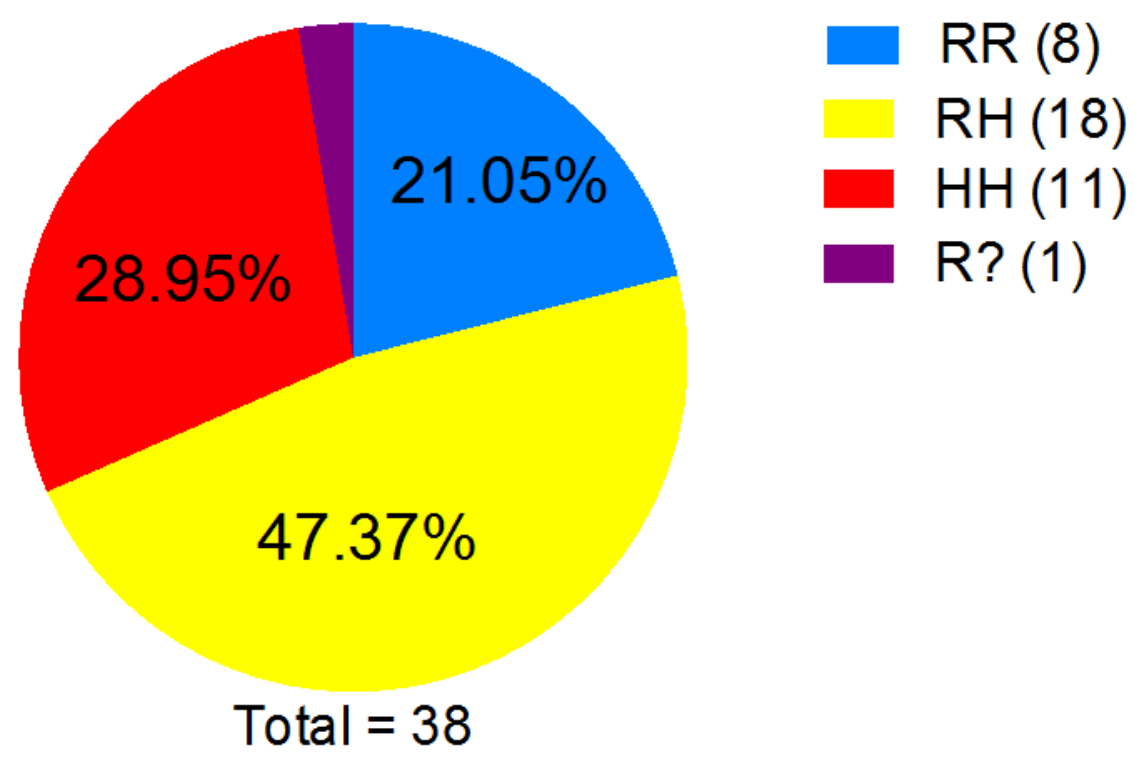

Figure 34: Incidence of rs1143679 in Louisville, KY area SLE Patients. Whole blood was obtained from SLE patients at the University of Louisville's Nephrology Clinic.

DNA was extracted, and PCR was performed for the rs1134679 allele, as described in the methods section. Example PCR results (top) and the incidence of R77H alleles in the cohort (bottom) are shown. A 304 base-pair band indicates the normal allele "R", while a 328 base-pair band signifies the mutant " $\mathrm{H}$ " form. 

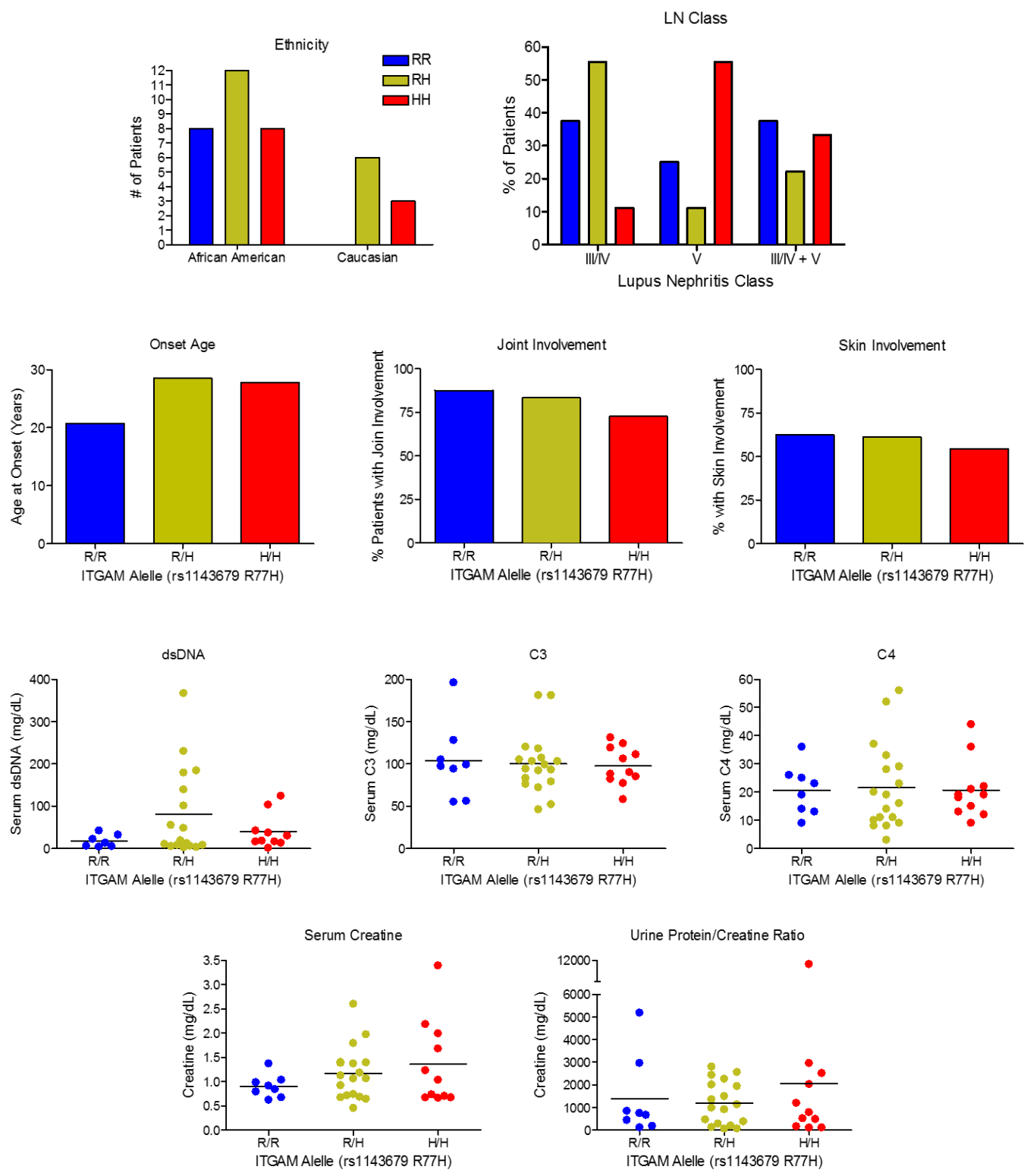

Figure 35: Clinical and demographic associations of rs1143679 in SLE. Clinical data from patients homozygous for mutant allele $(\mathrm{HH}, \mathrm{red})$ were compared with patients carrying a single copy (RH, yellow) or no mutation (RR, blue). ${ }^{*} \mathrm{P}<0.05$ (unpaired twotailed Student's t-test). 


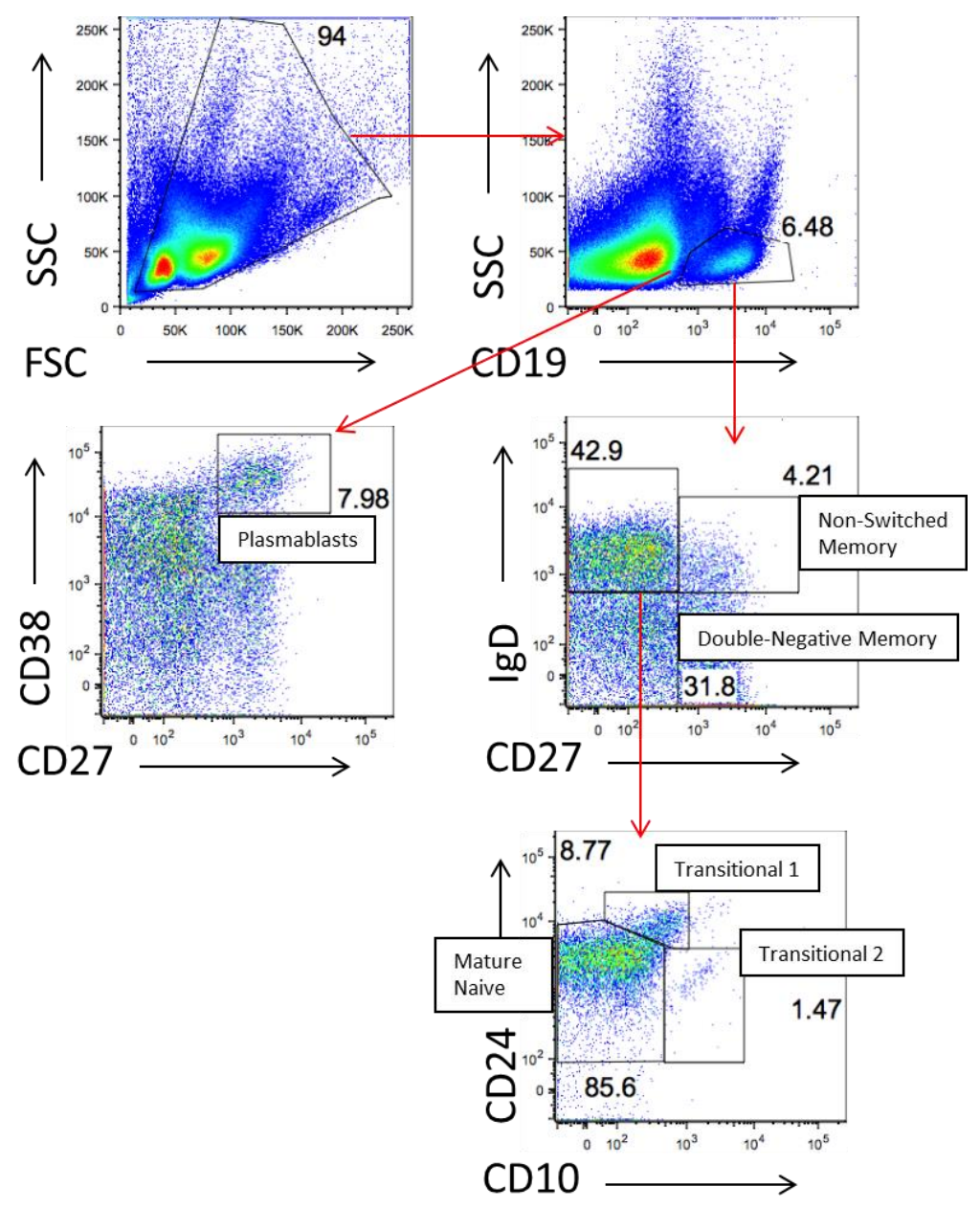

Figure 36: Identification of peripheral B cell subsets in SLE patients. Purified PBMCs from SLE patients were stained with antibody to identify B cell subsets. All populations were gated on $\mathrm{CD} 19^{+}$and identified as follows. Plasmablasts: $\mathrm{CD} 38^{+} \mathrm{CD} 27^{+}$, non-switched memory: $\mathrm{CD} 27^{+} \operatorname{IgD}^{+}$, double-negative memory: $\mathrm{CD} 27^{-} \mathrm{IgD}^{-}$, Mature Naïve: IgD ${ }^{+} \mathrm{CD} 27^{-} \mathrm{CD} 24^{+} \mathrm{CD} 10^{-}$, Transitional 1: $\operatorname{IgD}^{+} \mathrm{CD} 27^{-} \mathrm{CD} 24^{\mathrm{hi}} \mathrm{CD} 10^{-}$, Transitional 2: $\mathrm{IgD}^{+} \mathrm{CD} 27^{-} \mathrm{CD} 24^{+} \mathrm{CD} 10^{+}$. 

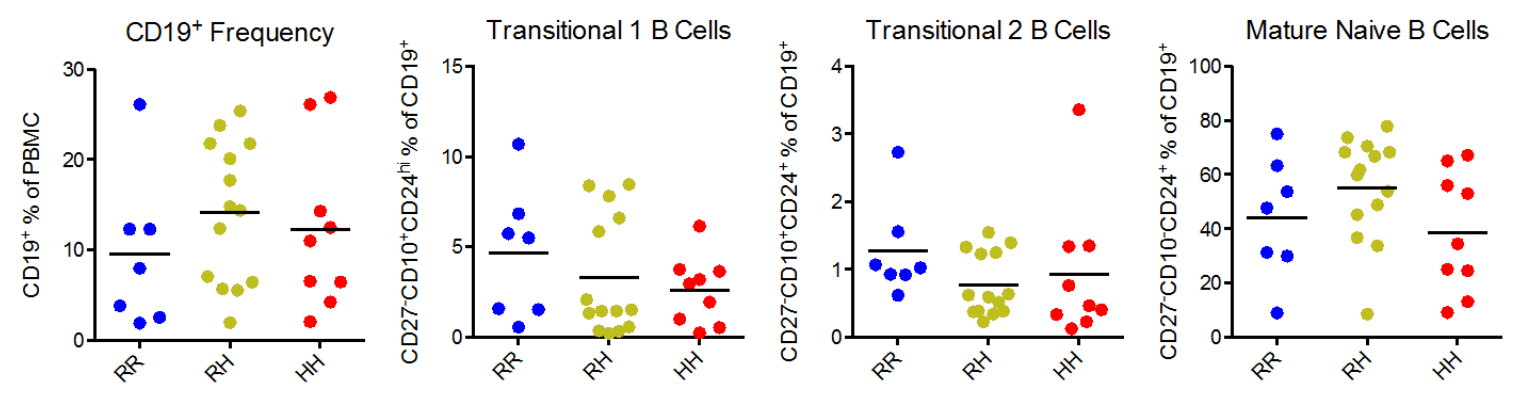

R77H Genotype

R77H Genotype

R77H Genotype

R77H Genotype

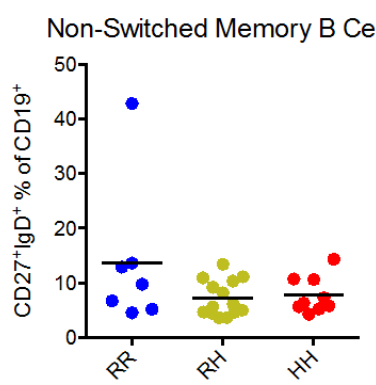

R77H Genotype

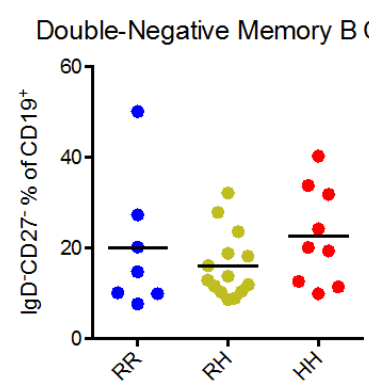

R77H Genotype

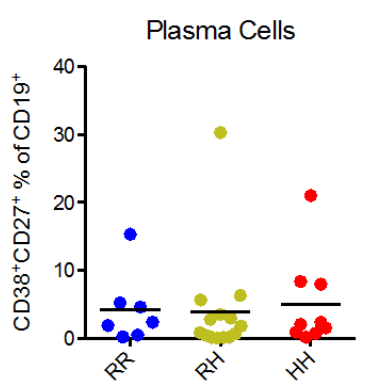

R77H Genotype

Figure 37: Peripheral B cell subsets in SLE patients with ITGAM mutation. Purified PBMCs from SLE patients were stained with antibody to identify B cell subsets, as described in the previous figure. Summarized data is shown. 

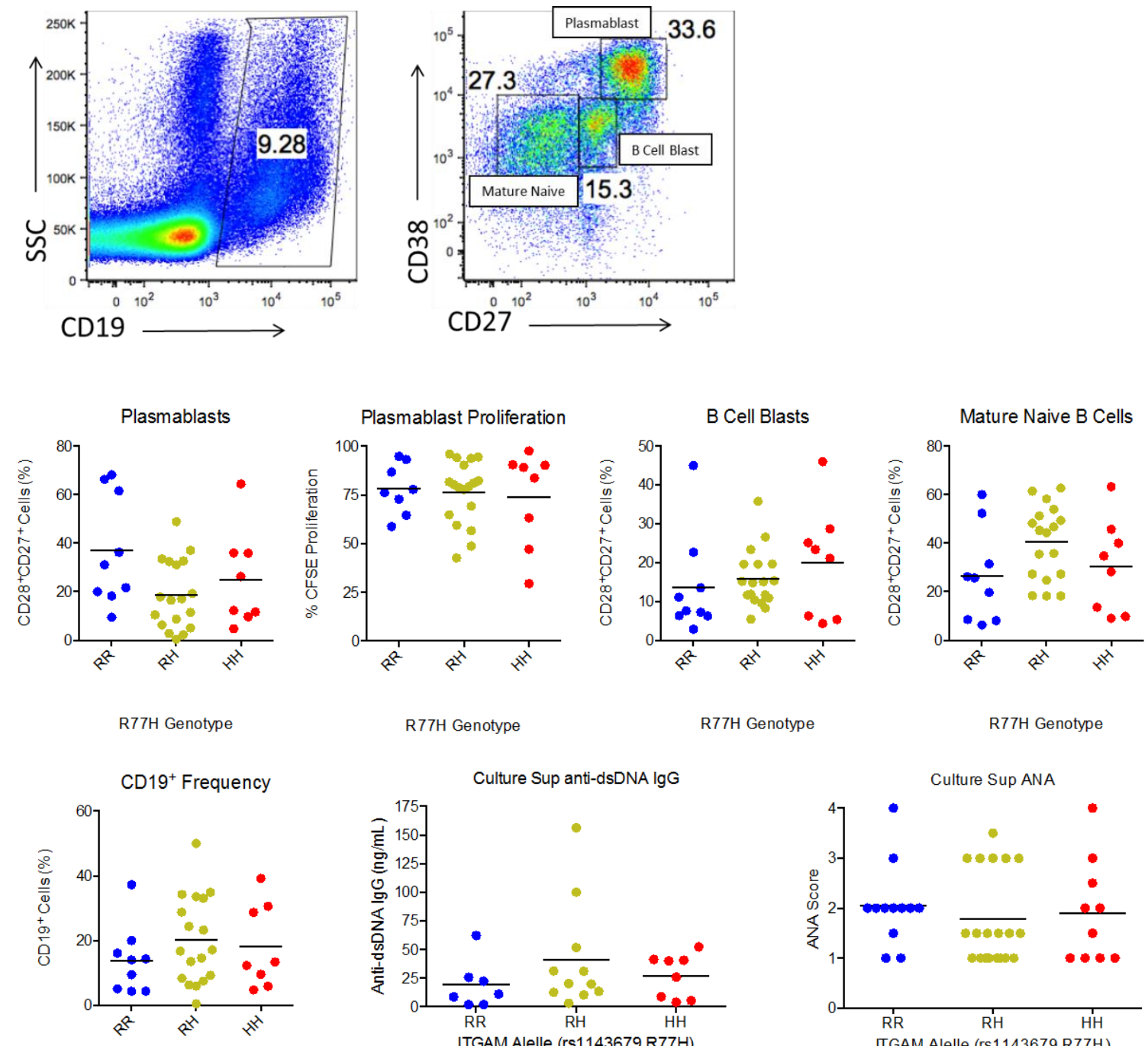

R77H Genotype

R77H Genotype

R77H Genotype
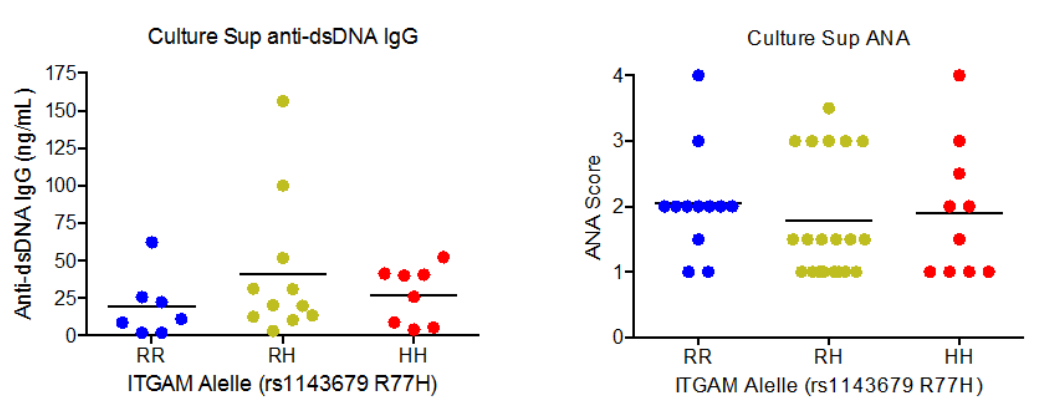

R77H Genotype

Figure 38: In vitro differentiation of plasmablasts from SLE patient PBMCs. Whole

PBMCs from SLE patients were labeled with carboxyfluorescein succinimidyl ester (CFSE) and cultured in the presence of $10 \mu \mathrm{g} / \mathrm{mL}$ anti- $\operatorname{IgM}, 0.33 \mathrm{nM} \mathrm{CpG}$, and $20 \mathrm{ng} / \mathrm{mL}$ hIL-21 for 7 days. Cells were stained with CD19, CD27, and CD38 to identify plasmablasts, B cell-blasts, and mature naïve cells as shown. CFSE signal was used to determine proliferation of the plasmablast population. Anti-dsDNA antibody and ANAs from the supernatant were measured via ELISA and microscopy score, respectively, Example dot-plot (top) and summarized data (bottom) are shown. 


\section{DISCUSSION}

The link between ITGAM mutations and SLE has been long-established. Previous research has focused on the impact of this mutation on the function of hallmark CD11b expressing cells. Indeed, these studies have found macrophage and dendritic cells carrying the $\mathrm{R} 77 \mathrm{H}$ form of $\mathrm{CD} 11 \mathrm{~b}$ have defects in phagocytosis, which is thought to contribute to SLE via the buildup of autoreactive antigen. However, our identification of $\mathrm{CD} 11 \mathrm{~b}$ as a BCR regulator has revealed B cells as a potential contributor to SLE pathogenesis in carriers of this mutation.

In our SLE cohort, we found a surprisingly high incidence of homozygous rs 1143679 at nearly $29 \%$. Previous studies have found $20 \%$ incidence in people of African descent, while European ancestry was at $17 \%$ (124). $23 \%$ of our African American patients carried the $\mathrm{HH}$ allele, while Caucasian patients had $33 \%$ incidence. However, this unusually high rate is likely due to a small sample size of 9 Caucasian patients. The prevalence of heterozygous carriers at $47 \%$ incidence was surprising, though the single mutant allele is unlikely to have any disease impact as previous work has found one functional gene provides a protective effect in neutrophils (247).

Association of ITGAM mutation and incidence of LN in Hong Kong Chinese, Thai, European, African American, and Latin American ancestry populations has previously been identified $(254,256-258)$. Our SLE cohort was unintentionally preselected for LN, as all patients were recruited via a nephrology clinic. Therefore, no such correlation could be examined. However, we were able to compare LN severity among R77H groups. Homozygous carriers and non-mutants had nearly equal numbers of patients with class 3,4 , and 5 nephritis. The heterozygous group had a proportionally 
higher amount of class 4 nephritis, but this is likely coincidental since the mutation is recessive, as discussed earlier. The association of ITGAM mutation with incidence, but not severity, of LN in SLE suggests an involvement in the initiation of kidney disease. We were able to detect a slight increase in the anti-dsDNA IgG titer of rs1143679 patients, which has also been reported before (256). Perhaps higher amounts of selfreactive antibody, resulting from increased B cell activity and decreased myeloid phagocytosis of nuclear antigen due to ITGAM dysfunction, is promoting a break in tolerance and initiation of kidney disease. The age of onset, incidence of joint involvement, and incidence of skin symptoms all showed no R77H correlation, similar to previous studies (259). Although CD11b functions as party of complement receptor 3 (CR3), no association of rs1143679 with C3 or C4 levels was found in our study or work by others (260). Reduction in C3 and C4 levels is a known indicator of SLE disease activity, but it appears they are not dependent on ITGAM function (261). Serum creatine levels were slightly increased in homozygous patients, but the creatine/urine protein ratio was not. Creatine is a classic biomarker of overall lupus severity, while the creatine/urine protein ratio is more specific to $\mathrm{LN}$ (262). It is possible that rs1143679 carriers do develop more server disease, but pre-selection for LN obscures and trend in the creatine/urine protein ratio. Our study was unable to reveal any novel correlations; thus, a larger cohort and a more comprehensive study of clinical biometrics may be required to better examine the effect of rs1143679 in LN and SLE.

Examination of the peripheral B cell subsets of our patient cohort surprisingly did not reveal any substantial association with ITGAM mutation. Pre-activation populations of transitional 1, transitional 2, and mature naïve B cells are likely upstream of any 
alteration of BCR activation caused by ITGAM dysfunction, and thus are unaffected. The lack of correlation in late stage memory B cells and plasmablasts with rs1143679 suggests that $\mathrm{CD} 11 \mathrm{~b}$ signaling alone is not enough to control disease. Indeed, our in vitro assays were also unable to produce a significant relationship of B cell response to this mutation. However, two major factors may be obscuring these results. Firstly, our cohort is both small in size and largely variable, most notably in the current disease treatment patients are under at the time of sample draw. Patients are receiving a wide range of different treatments, which can heavily influence the peripheral B cell populations. Our in vitro studies attempted to negate this effect by "washing out" any present drug influence, but the pre-selection of the B cell population by months or years of immunosuppression may have too large of an impact. Secondly, previous work has correlated rs1143679 with higher incidence of LN, but all of our patients were recruited via a nephrology clinic and thus were pre-selected with some amount of renal involvement. This screening of our patient population could be heavily skewing any correlation with clinical data or outcomes. The addition of an equal sized, non-nephritic cohort would allow for better study of this mutation's impact on disease. 


\section{CONCLUSIONARY REMARKS AND FUTURE PERSPECTIVES}

Much of the modern study of the immune system is focused on the identification and investigation of cell regulatory pathways. Treatments such as cancer immunotherapy, targeted immunosuppression, and the booming field of personalized medicine have all arisen from the discovery of novel immunoregulatory mechanisms that can be exploited to produce a favorable disease outcome. As the gaps in conventional knowledge become filled in, we must examine the unconventional and unexpected to further our understanding of immune system function.

Despite the identification of CD11b expressing B cell subsets over 25 years ago, its function in these cells has gone largely ignored. Classically considered a marker of myeloid cells, this low-key B cell population was passed over in favor of targets with more obvious potential. Previous findings showed the importance of CD11b in controlling autoimmunity and identified a functional role in B cells. In this study, we expanded this knowledge by demonstrating that CD11b acts as a controller of the healthy B cell response by regulating BCR activation signaling, therefore controlling the downstream generation of effector B cell subsets and immunogenic antibody. The ability lowly-expressed molecule to cause such a dramatic shift in response suggests either a potent ongoing regulatory effect, or acute regulation at a critical stage. Indeed, association of CD11b regulation with the CD22-Lyn-SHP1 circuit implicates participation in multiple regulatory mechanisms rather than restriction to a single 
pathway. It would be of interest to determine if there are stimulatory conditions, such as the presence of specific cytokines or certain types/sources of antigen, in which CD11bdependent regulation is ignored or disabled. Additionally, the actual mechanism of CD11b's regulatory effect is still unknown. The strong association of CD11b with CD22 suggests a critical stabilizing interaction which allows recruitment of SHP-1 and Lyn. Confirming whether this is through direct binding or is mediated through other molecules could reveal additional therapeutic targets of this mechanism.

The revelation of dynamic CD11b expression across B cell development implies an ability of these cells to control BCR regulation on a contextual basis. The inverse patterns of lowered and elevated CD11b expression following immunization in respective long-lived and short-term effector B cell subsets implies great complexities in not only CD11b control of BCR signaling, but cellular control of CD11b expression. It will be of great importance to determine precisely what factors are controlling the expression of CD11b, and whether they are shared among these divergent subset groups. The influence of different autoimmune models on CD11b in these cells is also very telling. Highly differential patterns between the Fas ${ }^{\text {lpr }}$ and ABIN1 models of lupus hints at an association of $\mathrm{CD} 11 \mathrm{~b}$ with autoreactive cell selection. Expression of this regulator may be relevant in the context of other diseases as well, and warrants pursuit.

Of particular note is the how many of the newly identified CD11b expressing B cell subsets are a relatively small fraction of the whole population. Most of these CD11 $\mathrm{b}^{+}$ sub-populations are less than $10 \%$ of the total cell type; however, they are distinctly defined by high CD11b instead of a low to intermediate level of expression. Several of our ex vivo experiments with CD11b knockout models have revealed defects in the entire 
B cell and GC B cell populations, clearly impacting a larger portion of cells than the small fractions identified in our reporter system. Determining whether these well-defined, higher CD11b expressing populations function differently than those with lower levels will reveal much about its role in controlling autoimmunity. Preliminary experiments have indicated this level of CD11b expression is indeed a permanent feature of these cells, rather than a transient shift amongst the entire B cell population. Indeed, ongoing studies to compare the repertoire of these defined CD11b expressing and non-expressing populations are currently underway. These studies can reveal a potential association of CD11b expression level with BCR specificity or self-reactivity.

Though our clinical research was not able to identify any novel correlations of ITGAM mutation with disease outcome or cell function, a more powerful study may be able to better answer these questions. In particular, examining the function of B cells from rs1134679 carrying healthy individuals would provide insights into the impact of $\mathrm{CD} 11 \mathrm{~b}$ function without the influence of disease state or long-term immunosuppressive treatment seen in our SLE cohort. It will also be vital to assess the expression pattern of $\mathrm{CD} 11 \mathrm{~b}$ in human B cell subsets, to determine the similarity to mouse models and the efficacy of their use. As part of our ongoing work, we will compare the peripheral B cell repertoire of ITGAM-variant and non-variant SLE patients, which can provide new insights on the impact of CD11b in B cell selection during disease.

In conclusion, these findings have confirmed the B cell-intrinsic effects of CD11b regulation on the humoral immune response and revealed the potential for dynamic functions of regulation across different stages of B cell maturity. Further research is necessary to delineate the exact purpose, mechanism of action, and impact of this 
regulation within each subset. The contribution of these regulatory processes to overall immune health can provide insight into the role B cell CD11b plays in human disease, and potentially reveal new therapeutic targets for treatment.

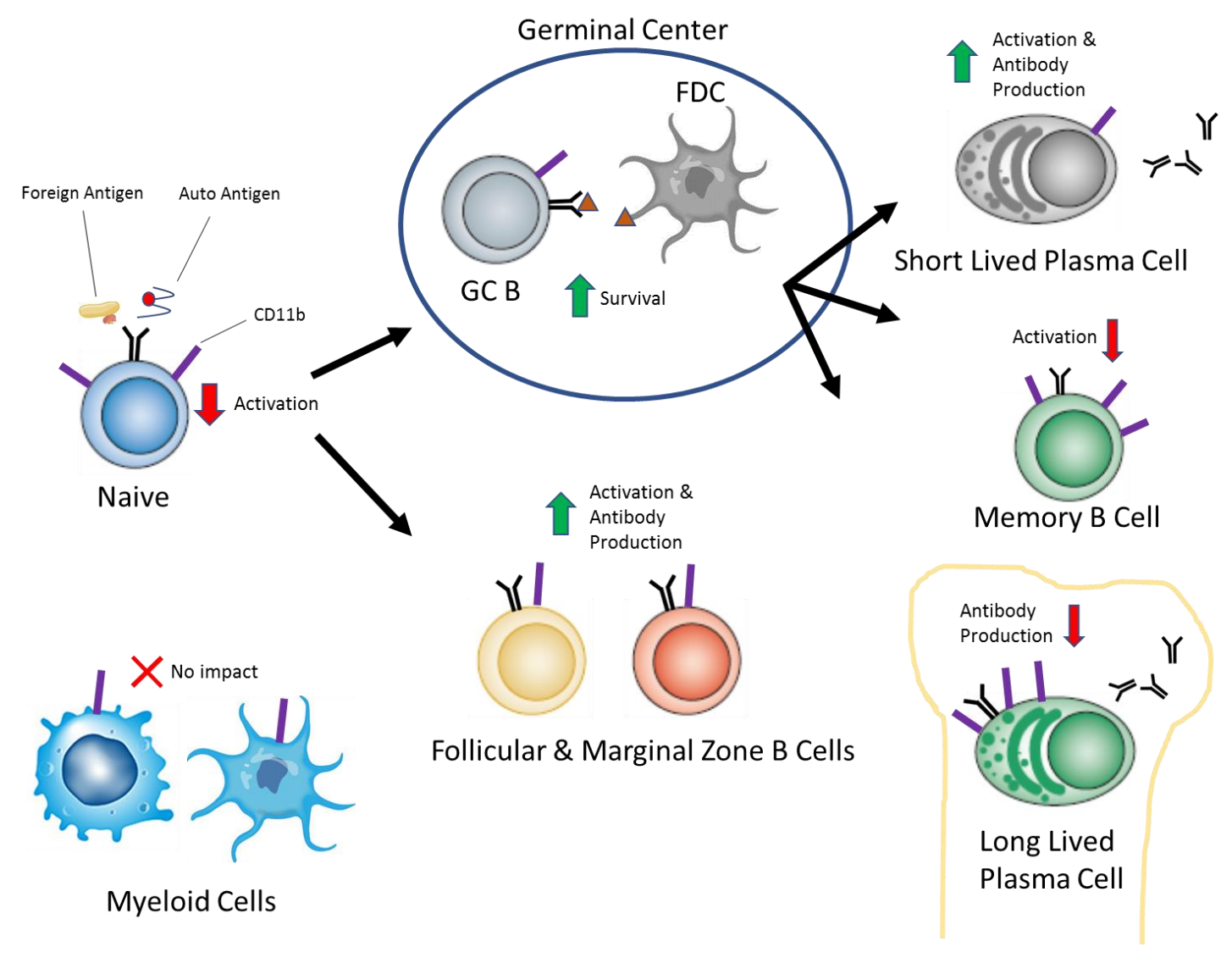

Figure 39: Graphical Abstract: The Impact of CD11b Regulation on B Cell Fate 


\section{CHAPTER IV}

\section{TRANSCRIPTION FACTOR STAT3 SERVES AS A NEGATIVE REGULATOR CONTROLLING IGE CLASS SWITCHING IN MICE ${ }^{1}$}

${ }^{1}$ This work has been accepted for publication in the following: Dascani et al "Transcription Factor STAT3 Serves as a Negative Regulator Controlling IgE Class Switching in Mice” ImmunoHorizons 2018 


\section{INTRODUCTION}

Production of the appropriate antibody class is a vital function of the B cell immune response. Immunoglobulin $\mathrm{E}(\mathrm{IgE})$ is likened with defense against parasitic infections (263). IgE is also largely associated with hypersensitivity and allergic disorders (264). Hyper IgE syndromes (HIES) are a group of immune disorders characterized by specific infectious susceptibilities, eosinophilia, and extremely high levels of IgE class antibody in the serum (265). Incidence of HIES has been linked to heterozygous mutations in the signal transducer and activator of transcription 3 (STAT3) gene (266, 267). However, precisely how transcription factor STAT3 contributes to the hyper-IgE phenotype has not been fully understood.

Class switching primarily occurs in the GC. In this unique microenvironment, recently activated $\mathrm{B}$ cells undergo class switch recombination and somatic hypermutation (SHM) to produce plasma cells which secrete high-affinity antibodies (268). STAT3 is well established as a major regulator of lymphocyte function, acting downstream of vital GC-related cytokines such as IL-4, IL-10, and IL-21 (269, 270). It has also been implicated as a critical factor for the development of T helper 2 (Th2) cells as well as follicular helper T cells, which are an essential component of the GC reaction $(271,272)$.

Studies using B cells from STAT3 loss of function HIES patients have revealed defects in many downstream effects of IL-21 and IL-10, including differentiation of antibody secreting cells $(273,274)$. Interestingly, these cells also demonstrate no alteration in their ability to class switch to $\operatorname{IgM}, \operatorname{IgG}$, and $\operatorname{IgA}$ forms (275). Reduced responses to vaccination and diminished memory B cell generation in these patients further suggests an important role for B cell function and antibody production (276). 
Despite these extensive findings, how exactly STAT3 controls IgE class switching remains largely unaddressed.

\section{Hyper IgE Syndrome}

Elevated level of immunoglobulin is a hallmark trait of several autoimmune disorders. In many of these diseases, such as SLE, effector IgG class antibody is the primary cause of pathogenicity. However, there are also many primary immunodeficiencies characterized by elevated levels of serum IgE, termed Hyper IgE Syndromes (277). HIES are often hallmarked by frequent and reoccurring infection of the lungs and skin by staphylococcus and fungal sources, as well as eczema and eosinophilia (278).

The study of this group of diseases has identified mutations in JAK-STAT molecules as the primary cause, attributing specific mutations to unique forms of the disorder. Autosomal-recessive (AR) forms of HIES are most often caused by null mutations in DOCK8, a GTPase activator (279). Though much less frequent, defects in PGM3 and TYK2 have also been linked to AR-HIES (280, 281). Interestingly, despite their similar functions, these different mutations cause distinct disease phenotypes. Patients with DOCK8 deficiency often have more severe mycobacterial and viral infections, whereas PGM3 is more strongly associated with neurocognitive symptoms (282).

Autosomal dominant (AD) HIES is the most common and well-studied form of hyper-IgE diseases. Also known as Job’s syndrome, AD-HIES was first described in 1966 by Davis et al. and identified by its hallmark eosinophilia, eczema, and recurring infections (278). The namesake elevated serum IgE was then identified by Buckley et al, 
coining the term HIES (283). Modern studies have since described these symptoms in more detail. Eczematoid rashes manifest only weeks after birth, usually around the scalp and face (284). Biopsy of these rashes reveals a Staphylococcus aureus driven eosinophilia, which can be resolved with antistaphylococcal therapy (285). Elevated serum IgE is also identified at their early stage, with titers of at least 2,000 IU/mL, if not higher (286). Recurring sinus and pulmonary infections can begin at 2-3 months of age, caused most frequently by $S$. aureus. These events can lead to permanent parenchymal lung damage and dangerous secondary infections that are a high source of morbidity and mortality in AD-HIES patients $(287,288)$. Patients are also susceptible to nonfilamentous molds such as pneumocystis jioveci leading to pneumonia, or cryptococcus in the intestine $(289,290)$.

In addition to these immunological symptoms, AD-HIES is also associated with manifestations involving dental, musculoskeletal, neurological, and vascular pathologies (291). In late childhood, facial patterns of asymmetry and coarse skin can be observed. Osteopenia and severe scoliosis may occur and require intense treatment (292). Abnormalities of the oral cavity are very common, as is failure to shed primary teeth without surgical intervention (293). Coronary artery aneurism is found in over $70 \%$ of AD-HIES patients, as was hypertension (294). Lastly, T2 hypersensitivity of the central nervous system are a common but poorly understood occurrence in this disease, and is often used as a diagnostic measure (295).

Despite a half-century of study, the genetic origin of AD-HIES was not identified until 2007 by Holland et al (296). This report examined a large cohort of AD-HIES patients found missense mutations in the $\mathrm{SH} 2$ and DNA-binding domains of STAT3. 
Notably, the mutations were autosomal dominant, thus establishing the unique AD-HIES form of hyper IgE syndrome. STAT3 expression was equal among mutant and wild-type forms, as was the ability of the molecule to phosphorylate and translocate to the nucleus (297). Since these initial studies, new findings have found that mutations specific to the SH2 domain do decrease the ability of STAT3 to phosphorylate at Tyrosine 705 (298). Despite these advances, the overall mechanism behind how these mutations impair STAT3 function has not been elucidated, particularly for polymorphisms to the DNA binding region. However, a recent study by Bocchini et al. has revealed that stabilization via increase chaperone protein activity is able to improve STAT3 function (299). This suggests a defect in post-activation stability is the mechanism behind STAT3's role in AD-HIES pathogenesis, but this warrant further investigation.

\section{STAT3 Signaling}

STAT3 was initially discovered and characterized by its ability to bind and control transcription of the IL-6 promoter region (300). Since then, the function of STAT3 as well as other STAT-family transcription factors has been well documented. STAT proteins are strongly associated with the Janus kinase-signal transducers (JAK) family signaling molecules. These JAK-STAT pathways are vital to many growth factor and cytokine signals which control a wide array of cell functions from proliferation and differentiation to survival and apoptosis $(301,302)$. Indeed, defects in these JAK-STAT signaling pathways are associated with multiple forms of autoimmune disorder and cancer.

The specific mechanisms of JAK-STAT signaling varies for each individual molecule, but the general cascade of events is conserved. The binding of surface receptor 
with extracellular ligand, such as a cytokine, causes dimerization of these receptor units. This event causes phosphorylation of the receptor cytoplasmic domain and subsequent recruitment of JAK proteins or induces phosphorylation of already present JAK molecules (303). The now activated JAK proteins then recruit and phosphorylate members of the STAT family in as homo or heterodimers, after which they translocate to the nucleus and activate target genes. STAT3 in particular is recruited by JAK1, JAK2, and TYK2 via phosphorylation on Tyrosine 705 (301).

As previously mentioned, STAT3 was first identified for its regulation of IL-6. Many more targets and activators of STAT3 have since been found. Some of the major cytokine activators of STAT3 include IL-6, IL-10, IL11-, IL-17, IL-21, and IL-22 (277). Downstream, STAT3 regulates an even greater array of genes including activation of IL6, IL-22, IL-26, IL17A, TGF- $\beta$, and MCP-1, and downregulation of TNF- $\alpha$, IL-12, and IFN- $\gamma(273,277,304,305)$. These signals are involved in a widely diverse set of cell functions, including both pro and anti-inflammatory responses. Indeed, STAT3 deficiency has been shown to impact both of these opposing forces in disease settings $(306,307)$.

Since it was first identified, much progress has been made on understanding the contribution of STAT3 deficiency to AD-HIES pathogenesis. Susceptibility to infection has been attributed to defects in the acute response driven by IL-6 (308). Likewise, defective IL-22 signaling can impair epithelial cell barriers which contributes to this effect (309). STAT3 mutations have also been shown to cripple the Th17 response, impacting CD4 T cell differentiation and neutrophil proliferation and chemotaxis. These deficiencies contribute to the reoccurring skin and lung infections in AD-HIES (310, 
311). On the anti-inflammatory side, mutations in STAT3 compromise its role in IL-10 signaling, resulting in increased inflammation in patients (312).

Predictably, antibody producing B cells are altered by STAT3 mutation in ADHIES, with the most obvious effect being increased production of IgE class antibody. Increased frequency of naïve and transitional B cells has also been noted in AD-HIES patients, along with decreased memory B cells (313). Surprisingly, T cells seem to be unaffected, implying the STAT3 driven B cell defect is not reliant on T cell help (314). In mice, loss IL-21 receptor, which utilizes STAT3, has been shown cooperate with IL-4 to negatively regulate production of IgE (315). Conversely, human studies have implicated IL-21 as an IgE inducer (316). Despite this obvious correlation with B cell $\operatorname{IgE}$ production, the specific relation and mechanism of STAT3 defects in regulating IgE antibody has not been previously studied.

\section{B Cell IgE Class Switching}

Upon engagement with cognate antigen, naïve B cells can enter the GC reaction to proliferate, increase affinity via SHM, and differentiate into antibody producing PCs; all of which were discussed in the previous chapter. Another vital process of the GC is class switch recombination. In CSR, B cells alter expression of the constant region of the immunoglobulin heavy chain ( $\operatorname{IgH})$ locus which defines the class of antibody produced. Located 3' from the variable $\mathrm{V}_{\mathrm{H}}, \mathrm{D}_{\mathrm{H}}$, and $\mathrm{J}_{\mathrm{H}}$ loci is the $\mathrm{C}_{\mathrm{H}}$ gene, comprised of multiple exons which each encode a different antibody constant region specific for an individual Ig class (317). Neighboring the upstream $\mathrm{V}_{\mathrm{J}}$ exon is the $\mathrm{C} \mu$ exon which encodes the "baseline" IgM class, while downstream lie the $\mathrm{C} \alpha, \mathrm{C} \varepsilon$, and $\mathrm{C} \gamma$ regions which encode the $\operatorname{IgA}, \operatorname{IgE}$, and $\operatorname{IgG}$ classes, respectively. Utilizing activation induced cytidine deaminase, 
the same enzyme used for SHM, GC B cells edit the germline to replace the default $\mathrm{C} \mu$ exon with one of the downstream regions, causing class switching (318).

Structure of the constant region of an antibody, determined by the germline, significantly impacts antigen affinity, and each class is utilized for specific types of antigen. Because of this, B cells class switching to each of the effector types is regulated to respond appropriately to immune insults. The IgE class results from the Th2 immune response, and is believed to have evolved to protect against parasite infections (319). Th2 type cytokines such as IL-4 and IL-13 have been shown to induce IgE class switching (320). Downstream of the cytokine receptor, STAT6 has been identified as a direct regulator of the IgE promoter, I $\varepsilon$. Acting as a homodimer, STAT6 coordinates promoter binding with other factors such as NF-kB, which can be induced by CD40-CD40L cosignaling or TLR stimulation (321).

Transcription of IE requires the presence of E2A proteins at the promoter region. However, expression of E2A is negatively regulated by Id2, which is constitutively activated under resting conditions. This mechanism serves to restrict IgE class switching and maintain typical low levels (compared to other antibody classes) of serum $\operatorname{IgE}$ (322). Interestingly, master GC regulator Bcl6 also negatively regulates IgE class switching by blocking the Is promoter (323). Despite the GC's role as the driver of class switching, the IgE form is negatively controlled by its most important regulator. This further emphasizes how tightly controlled the production of $\operatorname{IgE}$ antibody is. Several other molecules have been found to bind the I $\varepsilon$ promoter. AP-1 coordinates with STAT6 to initiate I $\varepsilon$ transcription (324). Nuclear factor interleukin-3 (NFIL3) has been found to increase IgE class switching. Induced by IL-4, NFIL3 binds directly to the Is promoter, 
and mice lacking this molecule demonstrate severely reduced IgE production (325). However, upstream regulation of NFIL3 expression, beyond IL-4 signaling, is still not understood.

IgE class switching is unique in that there are two pathways through with B cells can commit to this fate; direct and sequential switching. Direct switching is the traditional method, in which AICDA driven recombination removes loci upstream of I $\varepsilon$ in a single step and causes its expression. In sequential switching, B cells first undergo a class switch to $\mathrm{IgG}_{1}$ via expression of the $\mathrm{I} \gamma 1$ promoter. Then, during a second round of CSR, the gene is edited again to express the downstream I $\varepsilon$ which was not removed in the first step. This has been identified via the presence of $\mathrm{I} \gamma 1$ remnants present in $\operatorname{IgE}$ expressing B cells (326). More recently, it was shown that this sequential switch pathway is able to produce might higher affinity antibody than direct switching (327). These higher affinity IgE producing B cells may have a greater contribution to autoimmune disease than their lower affinity, direct switched counterparts.

Notably missing from these known contributors to IgE class switching is STAT3. The well documented link between STAT3 mutation and AD-HIES establishes a clear role for this transcription factor in control of $\operatorname{IgE}$, but this relationship has not been investigated. In this work, we seek to characterize the importance of STAT3 as an IgE class switch regulator and elucidate the mechanisms by which it acts. 


\section{MATERIALS AND METHODS}

Mice

C57BL/6 T and/or B cell STAT3 conditional knockout (cKO) mice $\left(\mathrm{STAT} 3^{\mathrm{fl} / \mathrm{fl}} \mathrm{CD} 2^{\mathrm{Cre} /+}\right.$ and STAT3 $3^{\mathrm{fl} / \mathrm{fl}} \mathrm{CD} 19^{\mathrm{Cre} /+}$ ) were generated by interbreeding STAT3 ${ }^{\mathrm{fl} / \mathrm{fl}}$ mice (328) (control) with $\mathrm{CD} 2^{\mathrm{Cre} / \mathrm{Cre}}$ or $\mathrm{CD} 19^{\mathrm{Cre} / \mathrm{Cre}}$ mice (The Jackson Laboratory), respectively. Mice at 6-8 weeks of age with both sexes were used for experiments. All mice were housed and bred in a conventional facility at the University of Louisville. Animal care and experiments were conducted in accordance with the National Institutes of Health guidelines and were approved by the Institutional Animal Care and Use Committee at the University of Louisville.

Immunizations and Ab Detection

STAT3 control or cKO mice were immunized by i.p. injection with $100 \mu \mathrm{g}$ of OVA in Alum adjuvant at a 1:1 ratio. Sera was collected before and at given time points after immunization. Anti-OVA antibody titers were measured via enzyme-linked immunosorbent assay (ELISA) using a 96-well plate coated with $1 \mu \mathrm{g}$ of OVA and a standard ELISAMAX kit (Biolegend, San Diego, CA). For IgE affinity detection, mice were immunized with $\mathrm{NP}_{23}-\mathrm{CGG}$ (Biosearch Technologies, Petaluma, CA). Sera was collected at day 14 post immunization. Anti-NP antibody was measured using parallel ELISAs in which wells were coated with $1 \mu \mathrm{g}$ of $\mathrm{NP}_{23}-\mathrm{BSA}$ or $\mathrm{NP}_{2}-\mathrm{BSA}$. The OD value ratio was used to determine overall affinity.

Abs and Flow Cytometry 
Fluorochrome-labeled mAbs against $\mathrm{IgG}_{1}, \mathrm{IgE}, \mathrm{B} 220, \mathrm{GL7}, \mathrm{CD} 138$, and viability detection antibody were purchased from BioLegend (San Diego, CA). Single-cell suspensions were blocked in the presence of anti-CD16/32 and purified anti-IgE at $4{ }^{\circ} \mathrm{C}$ for 15 minutes and stained on ice with appropriate Abs and isotype controls. For intracellular staining, cells were fixed and permeabilized followed by the addition of fluorochrome-labeled anti-IgE. Samples were acquired using a FACSCanto cytometer (BD Biosciences, San Jose, CA) and analyzed using FlowJo software (Tree Star, Ashaland, OR).

\section{Quantitative real-time PCR}

Splenic or iGB culture GC B cells and plasma cells were sorted by FACSAria III. Total RNA was prepared with TRIzol (Life Technologies) and RNeasy mini kit (Qiagen, Valencia, CA). After reverse transcription into cDNA with a reverse transcription kit (Bio-Rad, Hercules, CA), quantitative PCR was then performed on a Bio-rad MyiQ single-color RT-PCR detection system using SYBR Green Supermix (Bio-Rad). Primer sequences are listed in Supplemental Table 1. Gene expression was normalized to the $\beta_{2}-$ microblobulin $(\beta-\mathrm{MG})$ housekeeping gene and represented data as the fold differences by the $2^{-\Delta \Delta \mathrm{Ct}}$ method, where the threshold cycle $(\Delta \mathrm{Ct})=\mathrm{Ct}_{\text {arget gene }}-\mathrm{Ct}_{\beta-\mathrm{MG}}$ and $\Delta \Delta \mathrm{Ct}=$ $\Delta \mathrm{Ct}_{\text {induced }}-\Delta \mathrm{Ct}_{\text {reference }}$

In vitro-induced $G C B$ (iGB) Cell Culture

Purified B cells $\left(1 \times 10^{5}\right)$ from naïve STAT3 control or cKO mice were cultured in RPMI1640 medium (Sigma, St. Louis, MO) (supplemented with $10 \%$ FBS, 5.5x $10^{-5}$ M 2-ME, 
100 units $/ \mathrm{ml}^{-1}$ penicillin, and $100 \mu \mathrm{g} / \mathrm{mL}^{-1}$ streptomycin (GIBCO, Waltham, MA)) on a 12-well plate in the presence of CD40L and BAFF expressing feeder cells which had been irradiated with $120 \mathrm{G} \gamma \gamma$-rays (268). rIL-4 (1ng/mL, Peprotech, Rocky Hill, NJ) was added to the primary culture for 4 days. On day 4, cells were harvested, counted and used for secondary culture or analysis. For secondary culture, $2 \times 10^{5}$ cells from primary culture were re-plated on a new layer of irradiated CD40L feeder cells with 10ng/mL of rIL-21 for 3 days.

\section{STAT3 ChIP-seq and ChIP-qPCR}

In vitro cultured GC B cells from STAT3 control and cKO mice were used for ChIP studies as described previously (329). In brief, cells were subjected to sonication using a sonicator (AFA Focused ultrasonicator S220) to obtain chromatin fragments of 100-500 bp. Fragmented chromatin was incubated with STAT3 Ab (124H6, Cell Signaling Technology) or isotype control Ab and incubated for crosslinking with the beads (Dynabeads Protein G, Invitrogen). After crosslinking, crosslinks were reversed $\left(65^{\circ} \mathrm{C}\right.$ for 12-16 h), and precipitated DNA was treated with Proteinase K and then purified (QIAquick PCR purification kit, Qiagen). The DNA libraries were prepared, and sequencing was performed by BGI (Beijing Genomics Institute). The number of clean reads is approximately 24 million. The ChIP-seq data have been deposited into NCBI GEO with the accession number (GSE120022). The primer sequences of Bcl3, Crtc2, Bach2, Stat4, and Stim1 for ChIP-qPCR analysis were listed in the Supplemental Table 1. For STAT3-binding motif analysis, the overlapping regions between the two differential peak lists were ranked by the differences between control and cKO reads, and peaks that 
had fewer than 10 more reads were removed. The sequences were used as input into the MEME motif discovery software, using the oops model (one occurrence per sequence).

\section{OVA-Inhalation Asthma Model}

Control or cKO mice were immunized by i.p. injection of $25 \mu \mathrm{g}$ of OVA and $1 \mathrm{mg}$ Alum adjuvant, then boosted with the same treatment 14 days later. On day 21 post initial immunization, mice were anesthetized with isoflurane and challenged intranasally with $250 \mu \mathrm{g}$ of OVA in $30 \mu \mathrm{L}$ PBS. Intranasal challenge was repeated on days 22 and 23 , then mice were sacrificed on day 24. For microscopy, lobes of the lung were filled with and submerged in blocks of optimal cutting temperature (OCT) compound before rapidfreezing at $-140^{\circ} \mathrm{C}$ for storage.

\section{Immunofluorescence and Immunohistochemistry Staining}

Lung cryosections in OCT were sliced $9 \mu \mathrm{m}$ thick and fixed in acetone. For immunofluorescence, slices were blocked, stained with fluorescently labeled antibodies FITC-CD11b (Biolegend) and APC-CD193 (Biolegend) at 1:100 dilution overnight, then washed and stained with DAPI nuclear dye for 10 minutes before final wash and mounting. Images were acquired using a confocal microscope at 60x magnification (Nikon). For immunohistochemistry, slices were blocked overnight, stained with purified Gr-1 antibody at 1:50 dilution overnight. Slides were then washed and stained with secondary HRP-labeled antibody (Biolegend) at 1:1500 dilution for 25 minutes, washed and developed with peroxidase substrate (VECTOR Laboratories) for 10 minutes, then washed and counterstained with QS hematoxylin (VECTOR Laboratories) for 45 seconds 
before final mounting. Images were acquired using Aperio Scanscope digital scanner at 40x magnification.

\section{Statistical Analysis}

All quantitative data are shown as mean \pm s.e.m unless otherwise indicated. All samples were compared using two-tailed, unpaired Student's $T$ test. A $P$ value less than 0.05 was considered significant. Statistical analysis was performed with GraphPad Prism software. 


\section{RESULTS}

\section{STAT3 deficiency in B and T cell subsets disrupts the regulation of class switching}

\section{in a Th2-type immunization}

STAT3 has previously been shown to play a role in the formation of the GC following immunization (330). Because $\mathrm{B}$ cell and $\mathrm{T}_{\mathrm{fh}}$ cell interactions are crucial to the generation of an effective humoral immune response, we used a CD2 promoter driven Cre to investigate the effect of loss of STAT3 in these populations during a Th2 type immunization (331). At 7 days post immunization, a difference in antigen-specific IgE antibody titer could not be observed in the serum. However, levels of IgG and subclass $\mathrm{IgG}_{1}$ were significantly reduced at this time point. By 14 days after immunization, a marked increase in IgE was observed in CD2-cre;STAT3 ${ }^{f l f l}$ mice. IgG and $\operatorname{IgG}_{1}$ also maintained their decreased titers to this period (Figure 40).

Deletion of STAT3 on B and T cells caused impairment in germinal center formation. At day 14, STAT3 KO mice had significantly reduced overall GC frequency in the spleen compared to littermate controls (Figure 41, top). Within the GC population, there was also a shift in antibody class expression mirroring that seen in the peripheral blood. The amount of intracellular IgE expressing GCs was significantly increased in STAT3 knockout mice. $\operatorname{IgG}_{1}$ expressing GCs appear to be slightly reduced although not statistically significant. Antibody-producing plasma cells were also affected by loss of STAT3. While the frequency of plasma cells in the spleen was not markedly altered, their expression of antibody class was (Figure 41, bottom). Knockout mice demonstrated a substantial increase in $\mathrm{IgE}$ producing plasma cells while those of the $\mathrm{IgG}_{1}$ isotype were significantly reduced. 
Due to this apparent disruption of the GC reaction, we opted to investigate the overall affinity of antibody produced in this model by immunizing mice with NP-CGG (332). Plates were coated with highly conjugated NP23-BSA which is able to bind low and high affinity anti-NP antibody, or a much lower ratio NP2-BSA which only binds high affinity antibody. An increased $\mathrm{NP}_{2} / \mathrm{NP}_{23}$ OD value ratio conveys a higher overall affinity of antiNP antibody produced (327). IgE antibody produced by STAT3 KO mice was significantly lower affinity than controls, indicating a clear defect in affinity maturation in the GC (Figure 42, left) Despite this decreased affinity, STAT3 KO mice still produced greater amounts of $\operatorname{IgE}$ as indicated by absolute absorbance. $\operatorname{IgG}_{1}$ affinity was also reduced in knockout mice (Fig 42, right). These data suggest a critical role for STAT3 in the regulation of IgE class switching within the GC reaction and its plasma cell differentiation. 

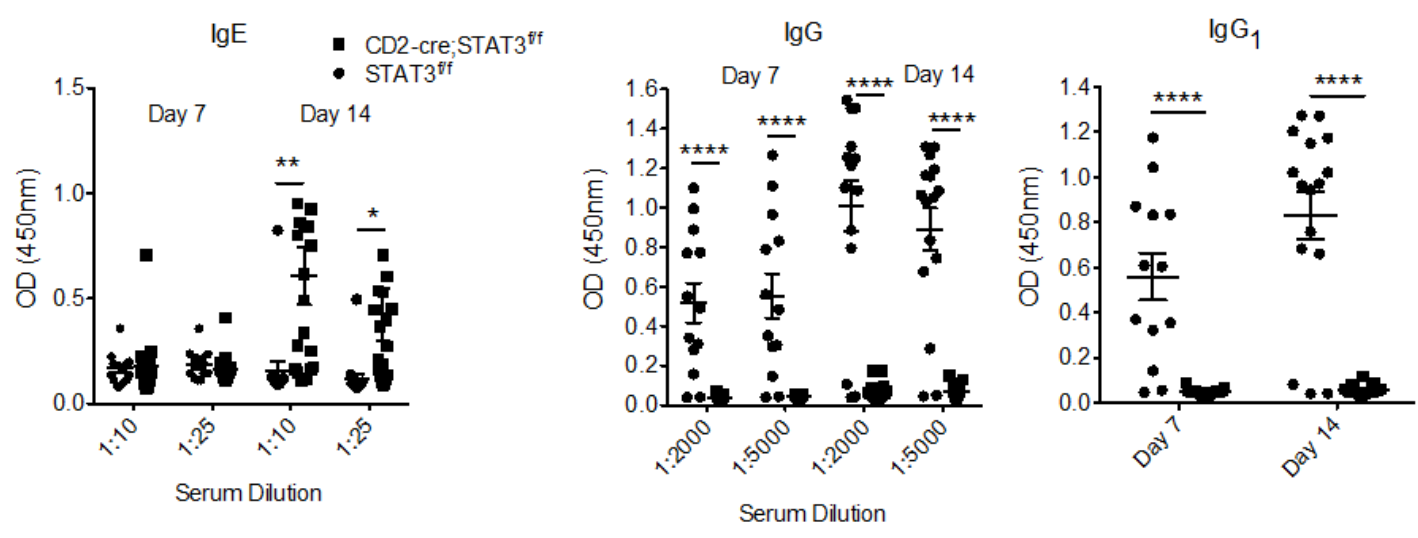

Figure 40: STAT3 regulates IgE antibody class switching in a Th2-type response. $\mathrm{STAT}^{\mathrm{f} / \mathrm{f}}$ control or CD2-Cre; STAT3 ${ }^{\mathrm{f} / \mathrm{f}}$ mice were immunized i.p. with $100 \mu \mathrm{g}$ OVA in Alum. Sera were collected at indicated time points and anti-OVA antibody levels of different classes and isotypes (IgE, $\operatorname{IgG}$, and $\left.\mathrm{IgG}_{1}\right)$ were detected by ELISA. Data are means \pm s.e.m* $P<0.05 ; * * P<0.01 ; * * * ; * * * * P<0.0001$ (unpaired two-tailed Student's $t$ test) 


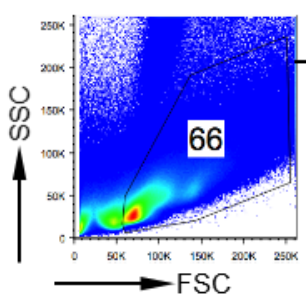

STAT3 ${ }^{\text {fF }}$

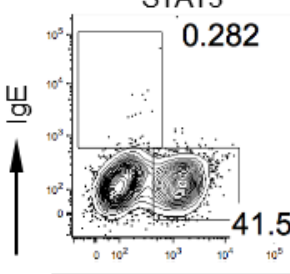

41.5
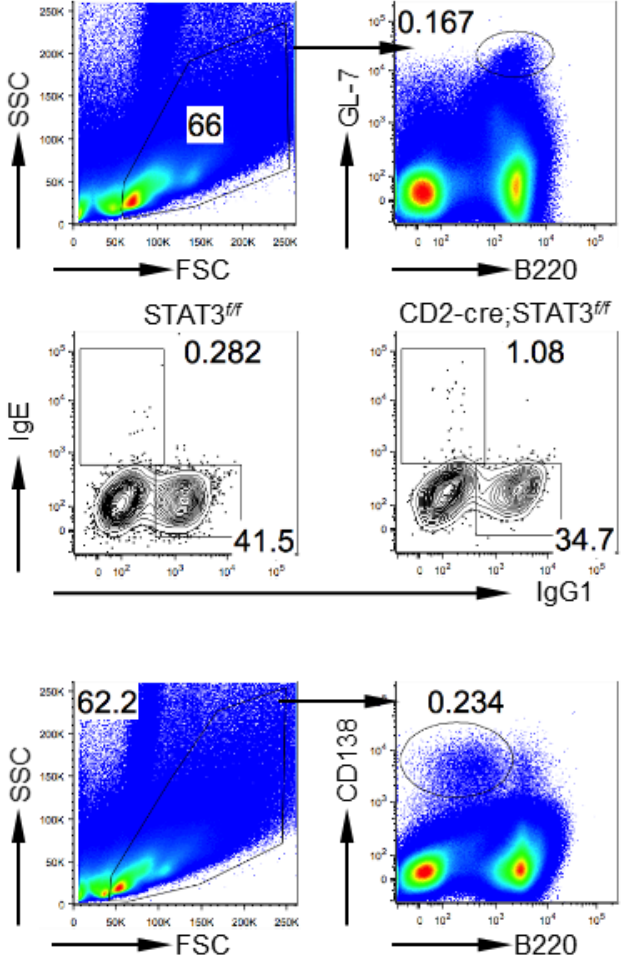

STAT3 $3^{\text {ff }}$

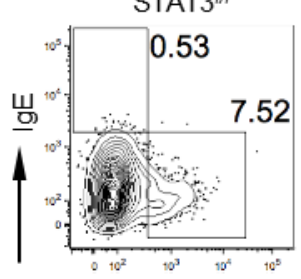

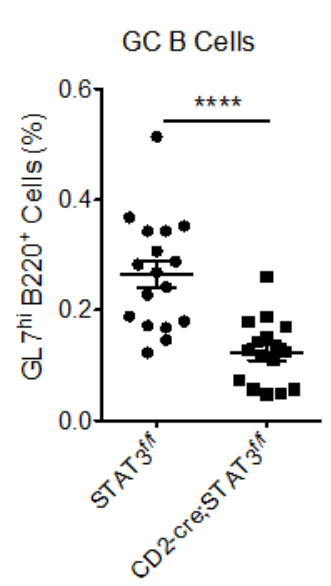
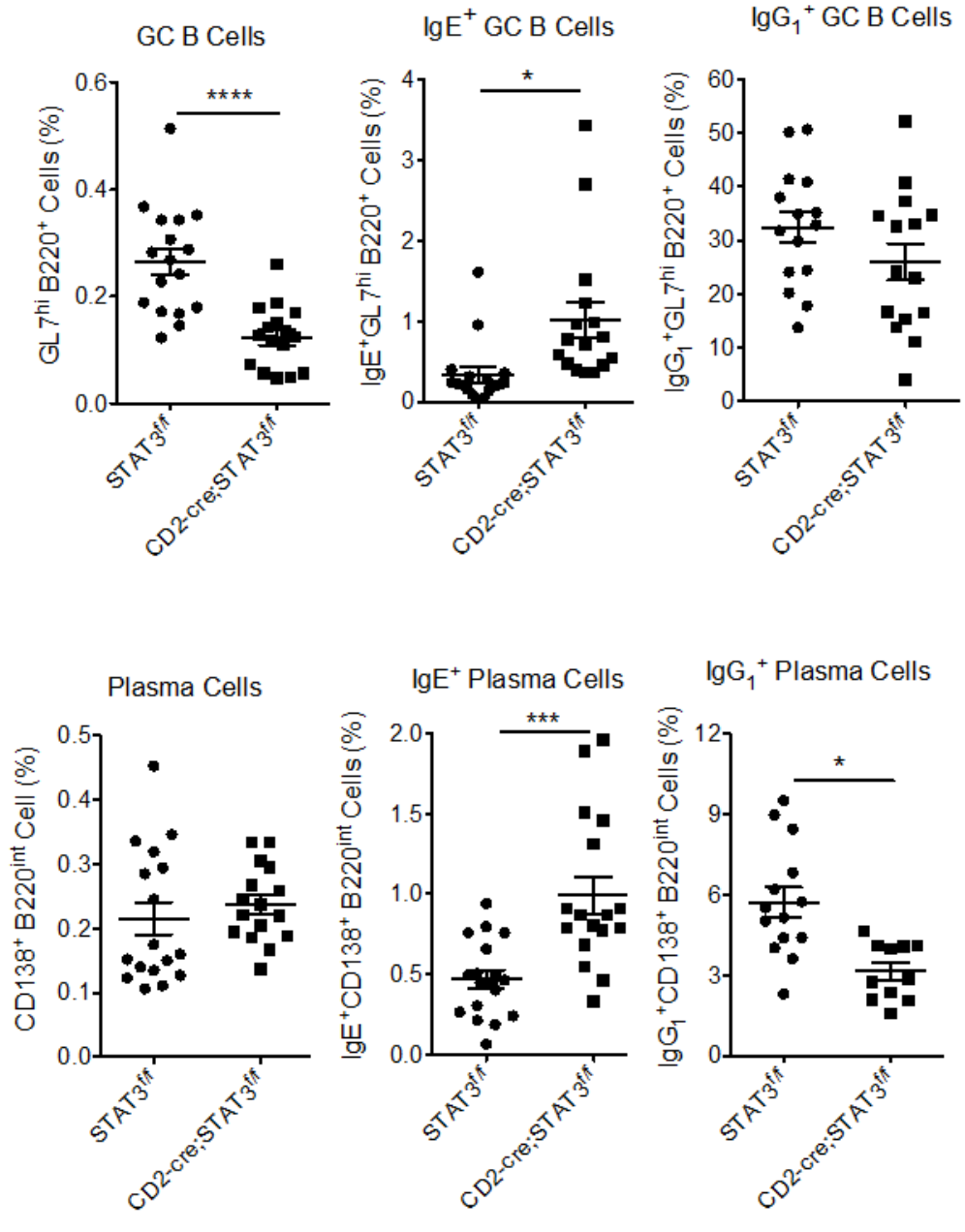

Figure 41: STAT3 regulates GC and PC IgE class switching in a Th2-type response.

$\mathrm{STAT}^{\mathrm{f} / \mathrm{f}}$ control or CD2-Cre; STAT3 ${ }^{\mathrm{f} / \mathrm{f}}$ mice were immunized i.p. with $100 \mu \mathrm{g}$ OVA in Alum. Spleens were harvested for FACS at day 14 post immunization. Cells were stained with mAbs B220, GL7, CD138, IgG , and intracellular IgE. Representative dot plots and summarized results are shown from at least three independent experiments. Data are means \pm s.e.m. ${ }^{*} P<0.05 ;{ }^{* * *} P<0.001 ; * * * * P<0.0001$ (unpaired two-tailed Student's $t$-test) 

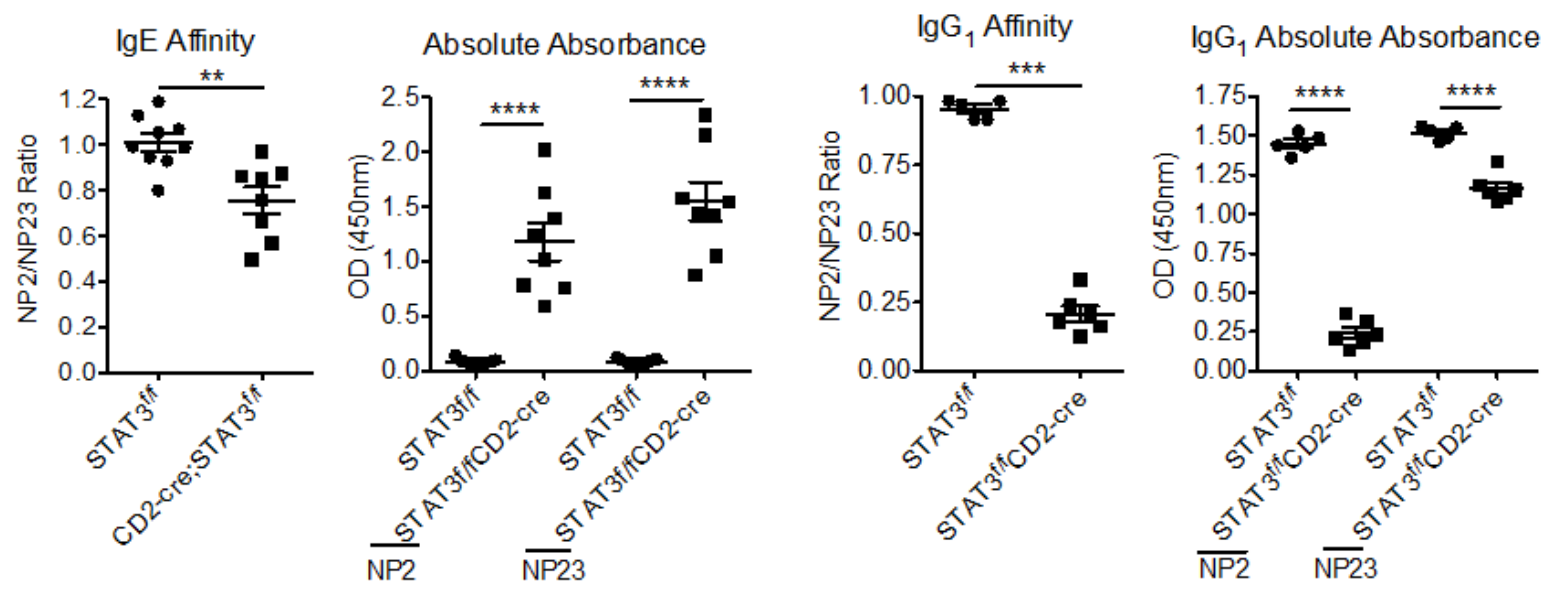

Figure 42: Loss of STAT3 leads to deceased antibody affinity in CD2-Cre driven

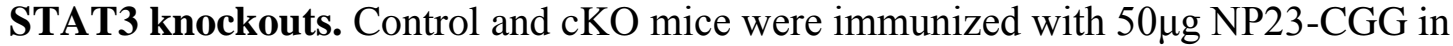
Alum via i.p. (A, B) Serum IgE (A) and IgG1 (B) were analyzed by ELISA with NP2BSA and NP23-BSA coated plates. Ratio of NP2 to NP23 absorbance (left) and absolute absorbance (right). Summarized results are from three independent experiments. Data are means \pm s.e.m $* * \mathrm{P}<0.01 ; * * * \mathrm{P}<0.001 ; * * * * \mathrm{P}<0.0001$ (unpaired two-tailed Student's ttest) 


\section{STAT3-dependent regulation of germinal center class switching is a B cell intrinsic}

effect

$\mathrm{T}_{\mathrm{fh}}$ and $\mathrm{B}$ cell interaction drives the formation and maintenance of the GC reaction.

However, it is B cells that make up the majority of the structure and undergo a more drastic amount of change and regulation (333). Thus, we sought to determine if the STAT3 dependent regulation of GC class switching was an effect specifically intrinsic to the B cell population. We used a CD19-Cre driven knockout model to examine these effects. In order to better understand the kinetics of this effect, multiple time points were observed. As early as day 7, antigen specific IgG antibodies in the serum were decreased, while IgE was elevated as seen in the CD2-Cre model (Figure 43). At day 14 these effects were even more pronounced. Additional isotypes were analyzed for this time point; demonstrating a decrease in serum $\operatorname{IgM}$ and a decrease in $\operatorname{IgG}$ subclasses $\operatorname{IgG}_{1}$, $\mathrm{IgG}_{2} \mathrm{a}$, and $\mathrm{IgG}_{2} \mathrm{~b}$. The affinity of day 14 antibody was also measured. Like the CD2-Cre model, both IgG and IgE anti-OVA antibody affinity was significantly reduced in knockout animals (Figure 44).

Analogous to the previous experiment, the progression of the GC reaction was also investigated. The GC is typically fully formed by 7 days post immunization $(333,334)$. Predictably, we found a marked and significant decrease in GC B cells at this time (Figure 45, top). Within the GC, cells switched to the IgE class were markedly increased. Similar to the later time point, $\mathrm{IgG}_{1}$ expressing GC frequency was also decreased. The plasma cells population was less impacted at day 7, showing no change in number or $\mathrm{IgG}_{1}$ class switching. The IgE expressing subset, however, increased (Figure 45, bottom). 
At 14 days post immunization these phenotypes became even more striking. The GC fraction was reduced in the absence of STAT3, while the IgE class subset was increased. The $\mathrm{IgG}_{1}$ subset was not altered at this time point (Figure 46, top). Plasma cells demonstrated the same marked increase in the IgE positive population but showed no other significant changes (Figure 46, bottom).

Within the GC structure, actively maturing GC B cells cycle between two distinct compartments. The light zone of the GC contains antigen presenting cells which drive selection of high affinity B cells through competition for survival signals, while the dark zone is the site of B cell proliferation and somatic hypermutation that results from successful light zone selection (335). We suspected that dysregulation of the migration of GC B cells between these two paradigms may be involved in the irregular class switching in the STAT3 deficient model. Analysis of CD86 and CXCR4 expression within the GC population demonstrates a slight increase in the dark zone fraction over the light zone in CD19-cre;STAT3 ${ }^{f l f l}$ mice (145) (Figure 47).

Transcription factor Bcl6 is a master regulator for GC function and formation (336, 337). Due to the decrease in GC formation in the STAT3 deficient mice, we sought to determine Bcl6 mRNA expression levels. Analysis showed lower Bcl6 expression in knockout mice compared to controls at day 14 (Figure 48). We also observed lower expression of Aicda, which is involved in somatic hypermutation and class-switch recombination in the GC (335). Next, we examined expression of IRF4 and IRF5, which have been linked to class-switch regulation and B cell differentiation, respectively (338, 339). These too were under-expressed in knockout mice. FOXO1, which is vital for the formation and maintenance of the dark zone stage of the germinal center reaction, was 
also downregulated in these cells (335). Interestingly, expression of dark zone chemokine receptor CXCR4 was not altered despite the observed difference in GC dark zone and light zone distribution. These data suggest STAT3 plays a key upstream regulatory role for several key genes in germinal center formation. 

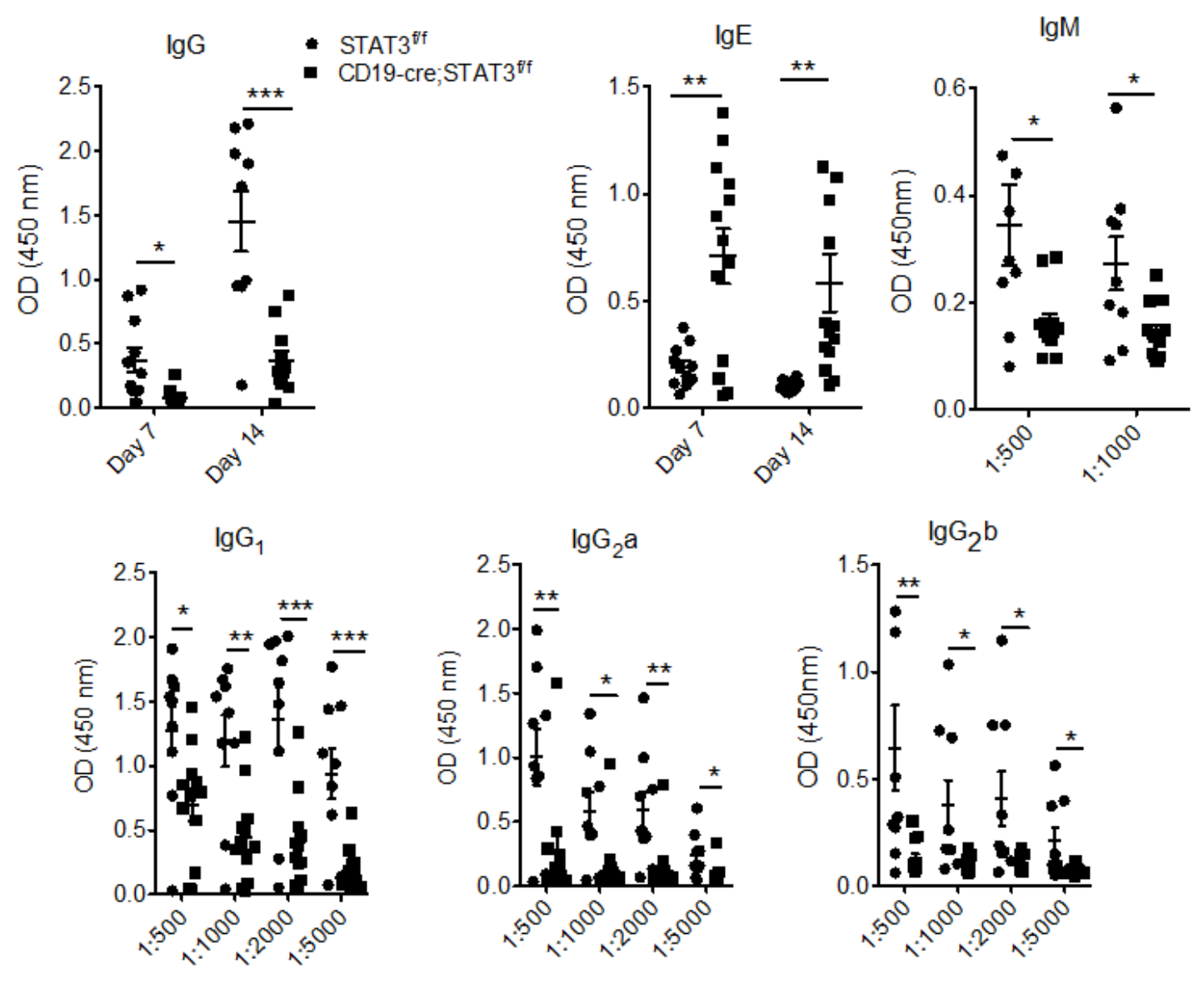

Figure 43: STAT3 regulation of class switching is a $\mathbf{B}$ cell intrinsic effect. STAT3 ${ }^{\mathrm{f} / \mathrm{f}}$ control or CD19-Cre; STAT3 ${ }^{\mathrm{f} / \mathrm{f}}+\mathrm{p}$. with $100 \mu \mathrm{g}$ OVA in Alum. Sera were collected, and anti-OVA antibody levels of different classes and isotypes were detected by ELISA at given dilutions. All data are from day 14 post-immunization unless otherwise noted. Summarized results are from at least three independent experiments. Data are means \pm s.e.m. $* P<0.05 ; * * P<0.01 ; * * * P<0.001 ; * * * * P<0.0001$ (unpaired two-tailed Student's $t$-test). 

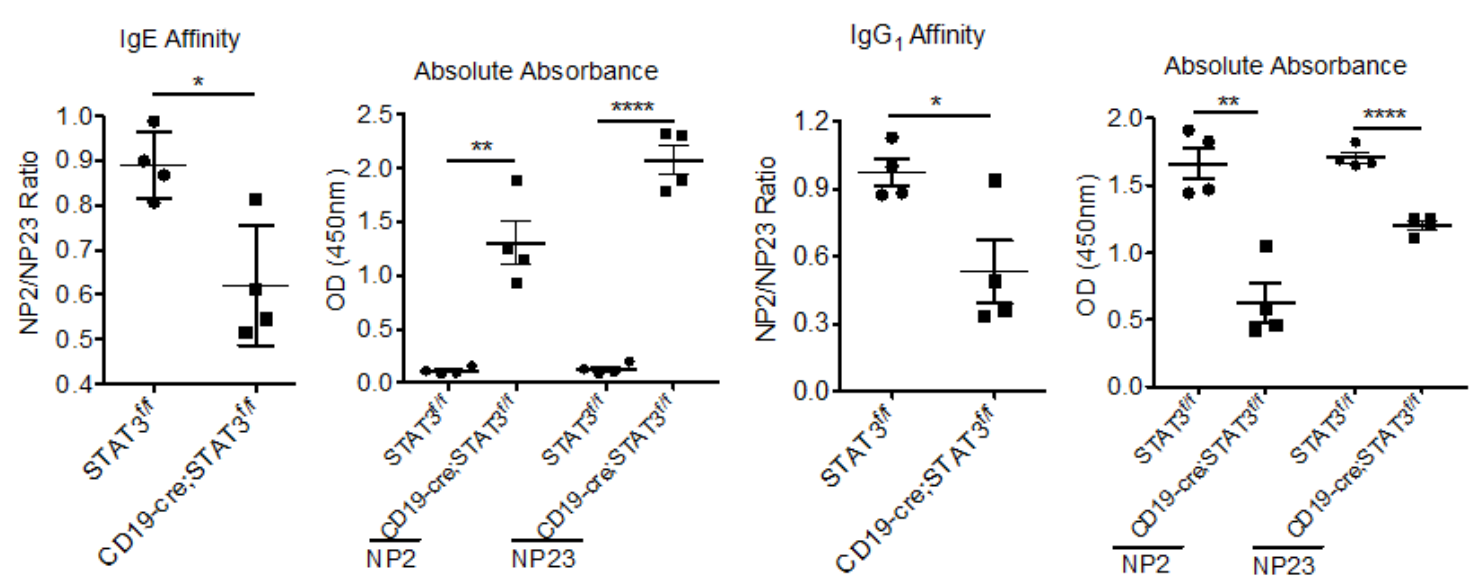

Figure 44: B cell STAT3 depletion alters antibody affinity Control and cKO mice were immunized with $50 \mu \mathrm{g} \mathrm{NP}_{23}-\mathrm{CGG}$ in Alum via i.p. Serum was collected day 14 post immunization. IgE and $\operatorname{IgG}_{1}$ were analyzed by ELISA with $\mathrm{NP}_{2}-\mathrm{BSA}$ and $\mathrm{NP}_{23}-\mathrm{BSA}$ coated plates. Ratio of NP2 to NP23 absorbance (left) and absolute absorbance (right) are shown. Data are means \pm s.e.m. ${ }^{* *} P<0.01$ (unpaired two-tailed Student's $t$-test) $* P<0.05$; $* * P<0.01 ; * * * * P<0.0001$ (unpaired two-tailed Student's $t$-test). 

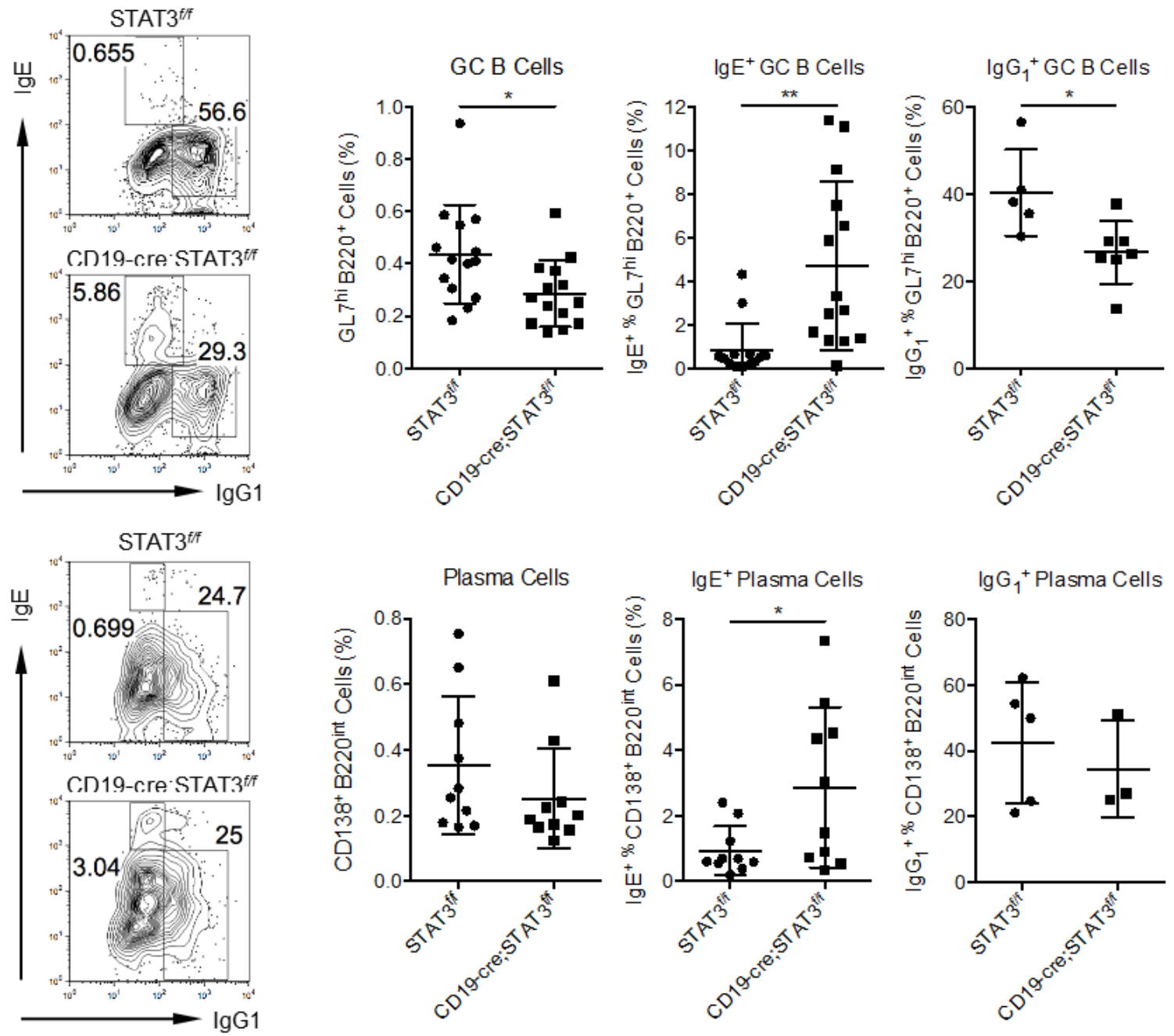

Figure 45: Loss of STAT3 in B cells alters early germinal center and plasma cell IgE class switching. STAT3f/f control or CD19-Cre; STAT3f/f mice were immunized i.p. with $100 \mu \mathrm{g}$ OVA in Alum. FACS analysis of splenocytes from day 7 post immunization mice. Cells were stained with mAbs B220, GL7, CD138, IgG1, and intracellular IgE. Representative dot plots and summarized results are from at least two independent experiments. Data are means \pm s.e.m. ${ }^{*} \mathrm{P}<0.05 ;{ }^{*} \mathrm{P}<0.01$ (unpaired two-tailed Student's ttest) 

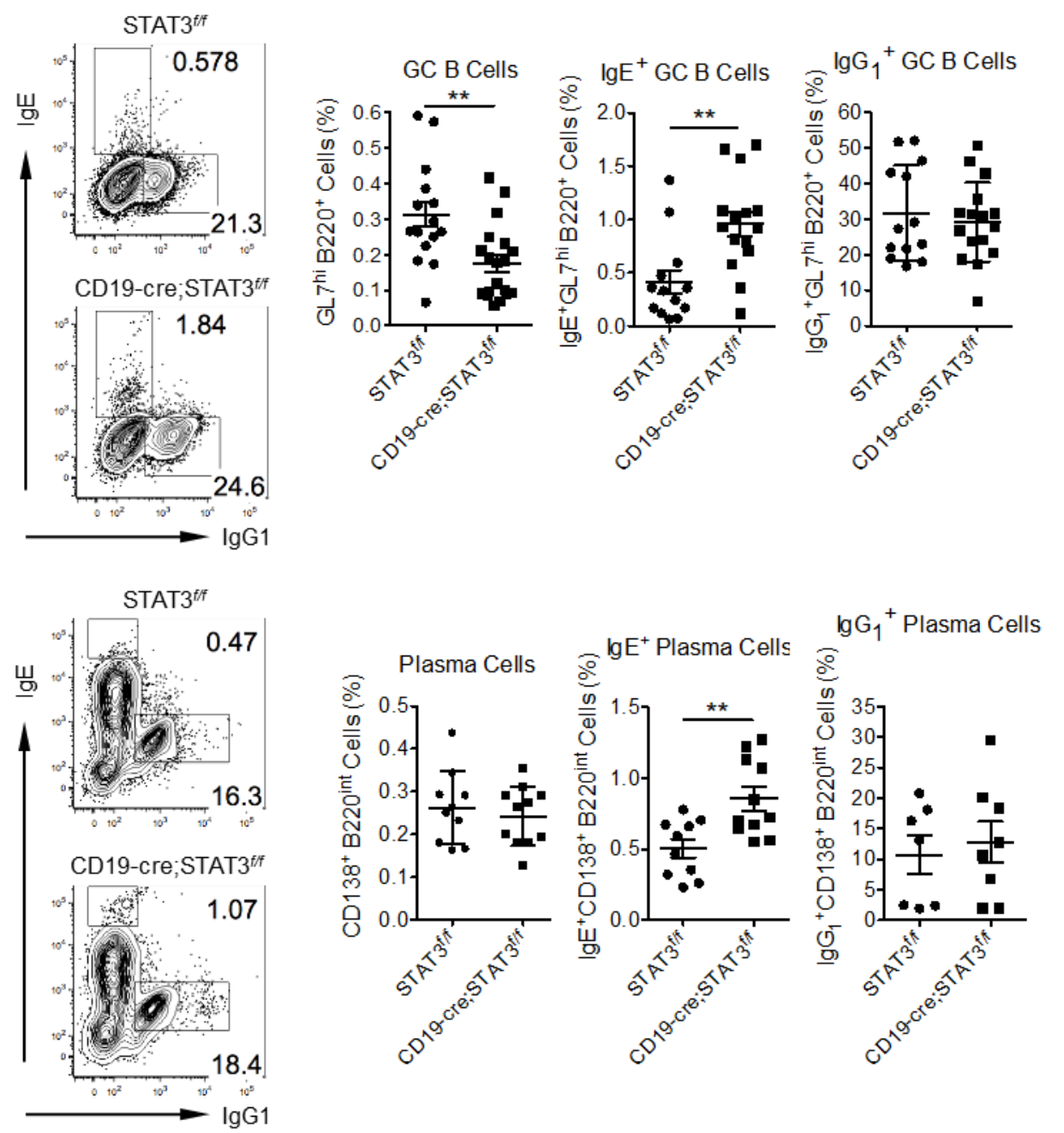

Figure 46: STAT3 regulates germinal center and plasma cell IgE class switching in B cells. STAT3 ${ }^{\mathrm{f} / \mathrm{f}}$ control or CD19-Cre; $\mathrm{STAT}^{\mathrm{f} / \mathrm{f}}$ mice were immunized i.p. with $100 \mu \mathrm{g}$ OVA in Alum. FACS analysis of splenocytes from day 14 post immunization mice. Cells were stained with mAbs B220, GL7, CD138, IgG $_{1}$, and intracellular IgE. Representative dot plots and summarized results are from at least three independent experiments. Data are means \pm s.e.m. ${ }^{* *} P<0.01$ (unpaired two-tailed Student's $t$-test) 

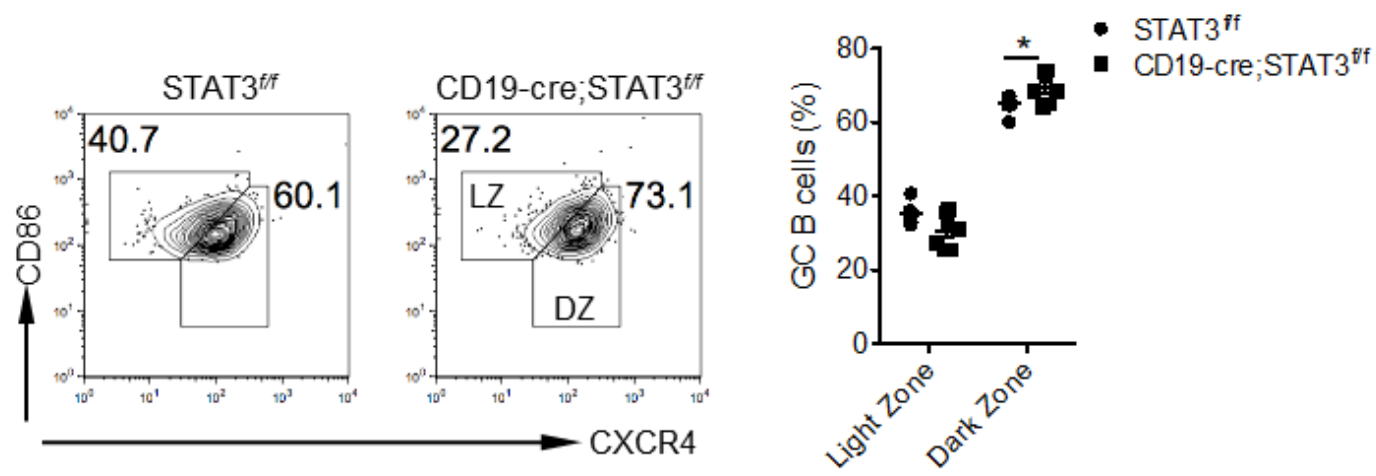

Figure 47: STAT3 depletion alters germinal center light zone and dark zone

distribution. STAT3 ${ }^{\mathrm{f} / \mathrm{f}}$ control or CD19-Cre; $\mathrm{STAT}^{\mathrm{f} / \mathrm{f}}$ mice were immunized i.p. with $100 \mu \mathrm{g}$ OVA in Alum. FACS analysis of splenocytes from day 14 post immunization mice.

FACS analysis of germinal center light zone and dark zone distribution. Cells were stained with mAbs B220, GL7, CXCR4 and CD86. Representative plots are gated on $\mathrm{B} 220^{+} \mathrm{GL}^{+}$. Summarized results are from two independent experiments. Data are means \pm s.e.m. ${ }^{*} P<0.05$ (unpaired two-tailed Student's $t$-test) 


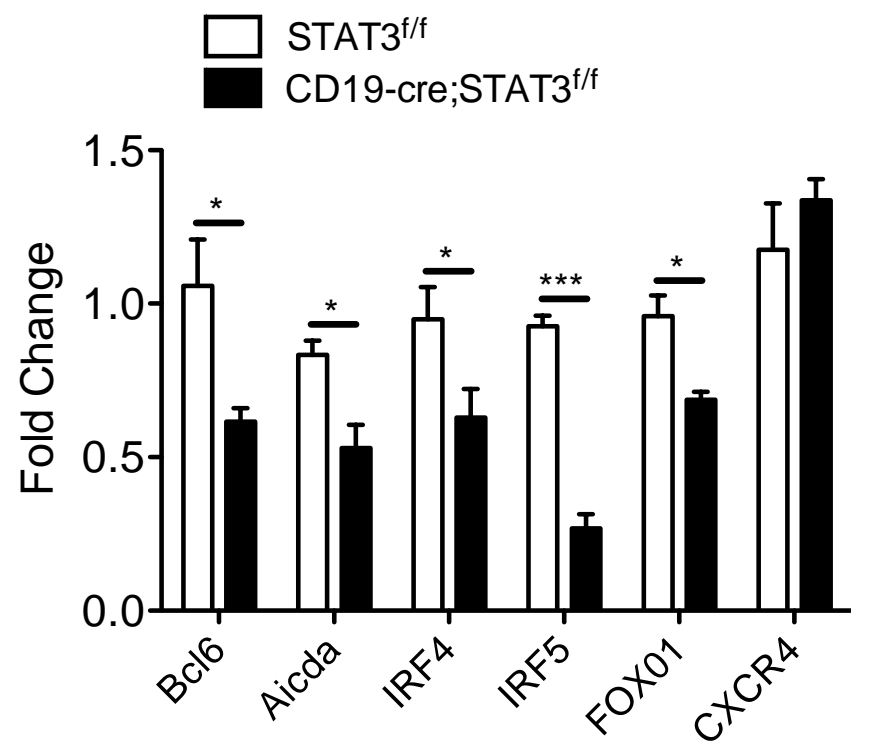

Figure 48: B cell deletion of STAT3 alters germinal center gene expression. GC gene expression of Bcl6, Aicda, IRF4, IRF5, FOXO1, and CXCR4 was measured using quantitative reverse transcriptase-PCR. GC cells were sorted from day 14 immunized WT or cKO mice. Data are means \pm s.e.m. $* P<0.05 ; * * * P<0.001$ (unpaired two-tailed Student's $t$-test) 


\section{Depletion of STAT3 regulates germinal center class switching in vitro.}

Previous studies have successfully established an in vitro system to replicate the in vivo GC reaction (268). We aimed to determine if the STAT3 deficiency in B cells would replicate the previously observed phenotype under these conditions. Culture of purified STAT3 knockout B cells in the presence of IL-4 and CD40L- and BAFFexpressing feeder cells produced fewer GC B-like cells at 96 hours, and induced less cell proliferation in general, but no changes in IgE expression (data not shown). Secondary culture with CD40L and IL-21 for 72 hours caused partial differentiation into plasma-like cells while maintaining a large portion of GC B-like cells. After this secondary differentiation, STAT3 knockout cultures maintained a reduced rate of overall expansion compared to controls (Figure 49). In addition, STAT3 knockout B cell culture continued to have reduced GC B-like frequency (Figure 50, top) However, these cells experienced greater $\operatorname{IgE}$ and fewer $\operatorname{IgG}_{1}$ class switching, mimicking the phenotype observed using in vivo Th2-driven immunization models. The plasma cell-like population also demonstrated similar results, displaying an increased overall frequency, increased $\operatorname{IgE}$ switching, and decreased $\operatorname{IgG}_{1}$ isotype subset (Figure 50, bottom). mRNA expression analyses were performed on the end-stage GC B-like and plasma cell-like populations to further confirm replication of the in vivo phenotype. GC B-like cells maintained significantly decreased expression of the germinal center genes Bcl6, Aicda, IRF4, IRF5, and FOXO1 (Figure 51, left). Unlike the in vivo GCs, CXCR4 expression was decreased in these cells. Plasma-like cells also mimicked an in vivo expression pattern, with decreased Aicda, IRF4, and IRF5 (Figure 51, right). These data confirm the in vivo effect of STAT3 deficiency seen in mice and suggest that this effect is 
a result of signaling via the IL-4, IL-21, or CD40 pathways used in the germinal center reaction. 

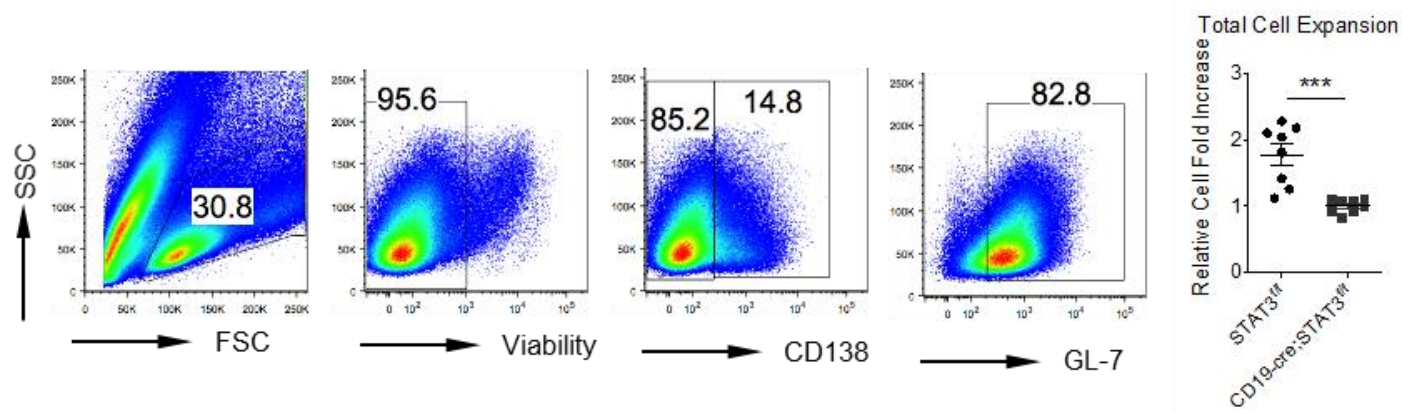

Figure 49: STAT3 regulates IgE class switching of in-vitro generated germinal

center B cells. Purified B cells from STAT3 control and cKO mice were cultured with CD40L and BAFF expressing feeder cells in the presence of IL-4 for 96 hours, followed by secondary culture with feeder cells and IL-21 for 72 hours. Total cell number from of secondary culture was evaluated by hemocytometer. Data are means \pm s.e.m. $* * * P<0.001$ (unpaired two-tailed Student's $t$-test) 

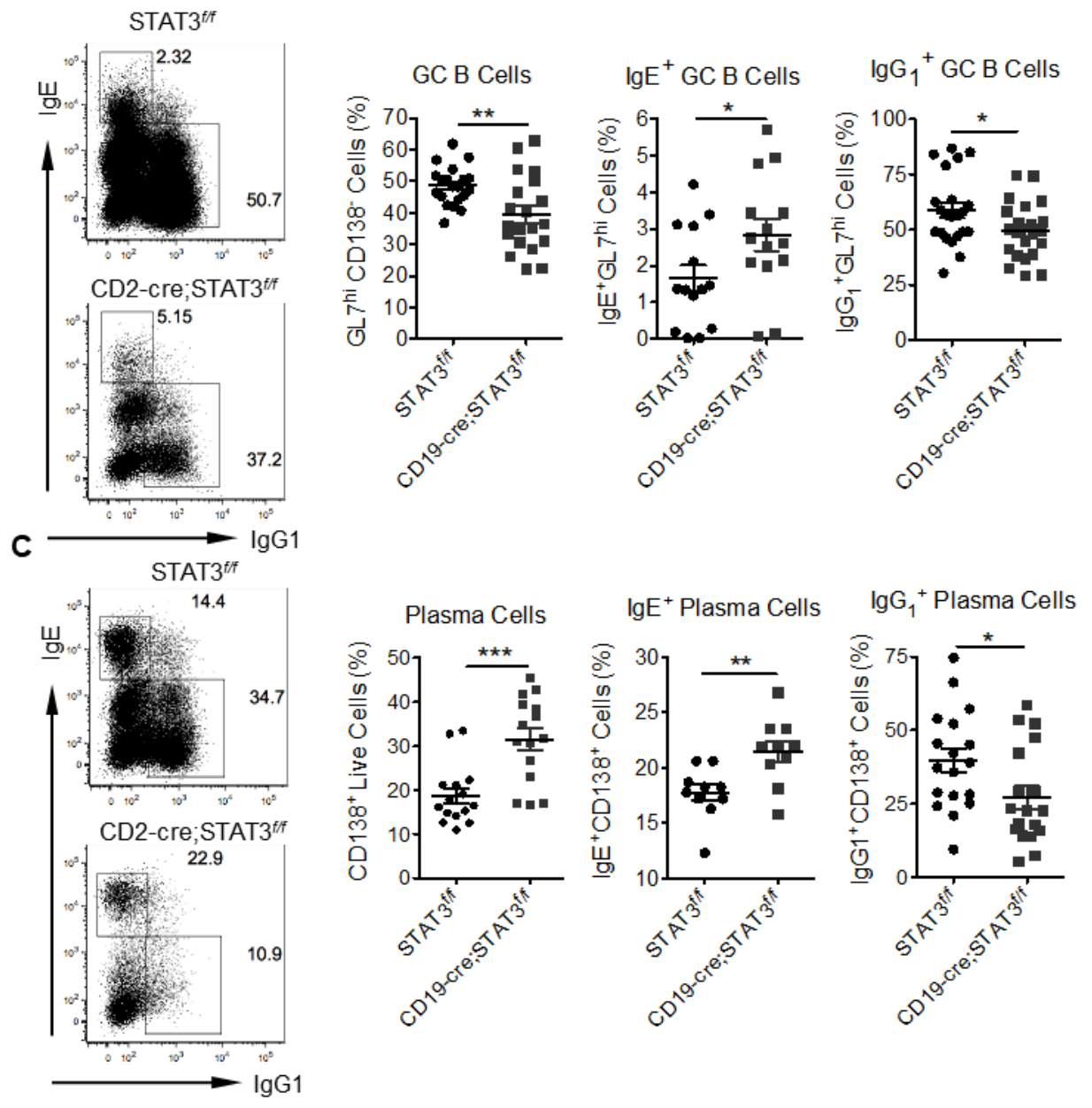

Figure 50: STAT3 regulates IgE class switching of in-vitro generated germinal

center B cells. Purified B cells from STAT3 control and cKO mice were cultured with CD40L and BAFF expressing feeder cells in the presence of IL-4 for 96 hours, followed by secondary culture with feeder cells and IL-21 for 72 hours. Cells were harvested and stained with mAbs CD138, GL7, IgG $_{1}$, and intracellular IgE. FACS analysis of iGB (top) and iPC (bottom) $\operatorname{IgG}_{1}$ and IgE expression. Representative plots are gated on CD138 $\mathrm{GL}^{+}$cells and $\mathrm{CD} 138^{+}$cells, as shown in the previous figure. Data are means \pm s.e.m. Summarized data are from at least three independent experiments. ${ }^{*} P<0.05 ; * * P<0.01$; $* * * P<0.001$ (unpaired two-tailed Student's $t$-test) 

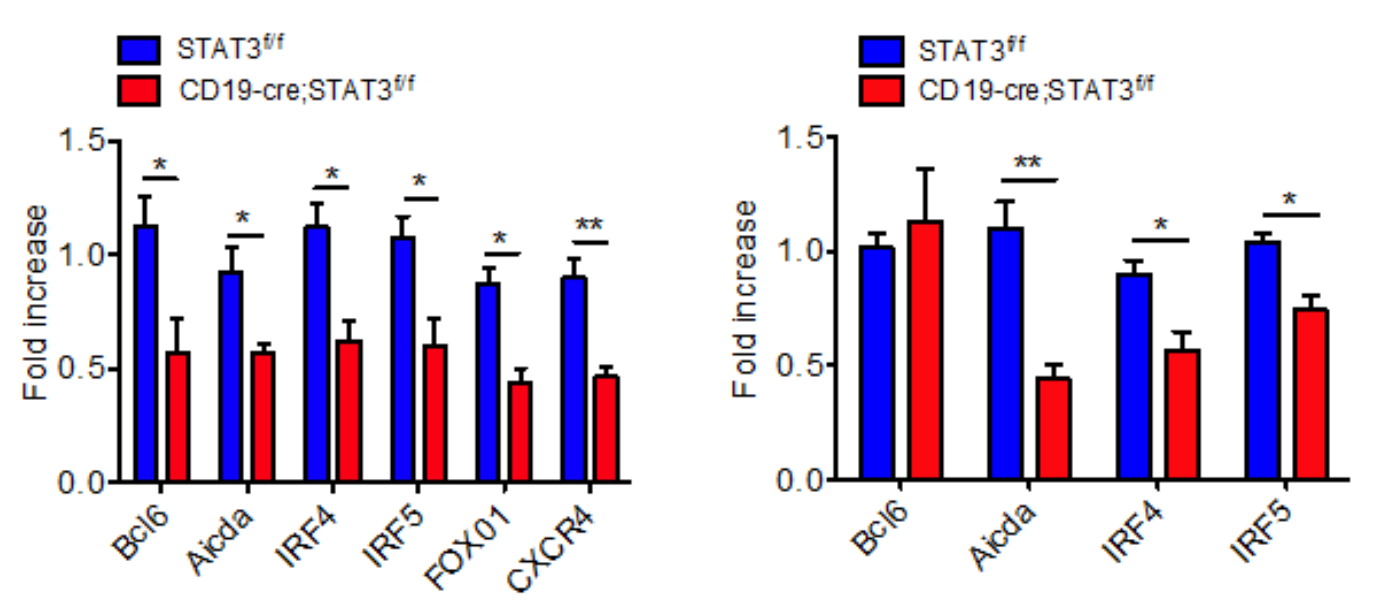

Figure 51: STAT3 regulates germinal center related genes in in vitro generated germinal center B cells. Gene expression of Bcl6, Aicda, IRF4, IRF5, FOXO1, and CXCR4 was measured using quantitative reverse transcriptase-PCR. Cells were sorted from day 7 cultured WT or cKO GC B-like (left) and plasma-like (right) cells. Data are means \pm s.e.m. Summarized data are from at least three independent experiments. ${ }^{*} P<0.05 ; * * P<0.01$ (unpaired two-tailed Student's $t$-test) 


\section{Target genes of STAT3 in the GC B cells}

To further identify the direct or indirect target genes of STAT3 in the GC B cells, a STAT3 chromatin immunoprecipitation sequencing (ChIP-seq) study was carried out. In total, 3571 STAT3-binding sites were identified, most of them were located in introns or intergenic regions (Figure 52, left), suggesting that STAT3 may regulate gene expression through binding to distal regulatory elements. Gene Ontology (GO) analysis of differential peak related genes suggested that these genes are related to biological process such as immune regulation and metabolic process, cellular component, and molecular function (Figure 53, top). Ranking of genes associated with STAT3 based on the absolute number of sequencing tags, $B c l 3$ and $C r t c 2$ were identified as the genes with the greater recruitment of STAT3 (Figure 52, right). In addition, Bach2, Stim1, and Stat4 had also binding site of STAT3 (data not shown). Interestingly, STAT3 itself was also found to be among the direct STAT3-regulated targets (data not shown).

To validate results from ChIP-seq experiments, five STAT3 bound genes identified by ChIP-seq, including Bcl3, Crtc2, Bach2, Stat4, and Stim1 were selected. Bcl3 and Crtc2 showed strong binding to STAT3 while Bach2, Stat4 and Stim1 showed weak to no binding using iGB from STAT3 control and cKO mice (Figure 54). This was also confirmed using iGB from WT mice immunoprecipitated with STAT3 mAb or isotype control mAb (Figure 53, bottom). We also searched STAT3 binding motif using differentially expressed sequences between STAT3 control and cKO GC B cells. A de novo STAT3 binding motif was discovered (Figure 52, bottom), suggesting that target genes with the STAT3-binding motif may be involved in STAT3 regulated GC B cell differentiation and IgE isotype switching. 

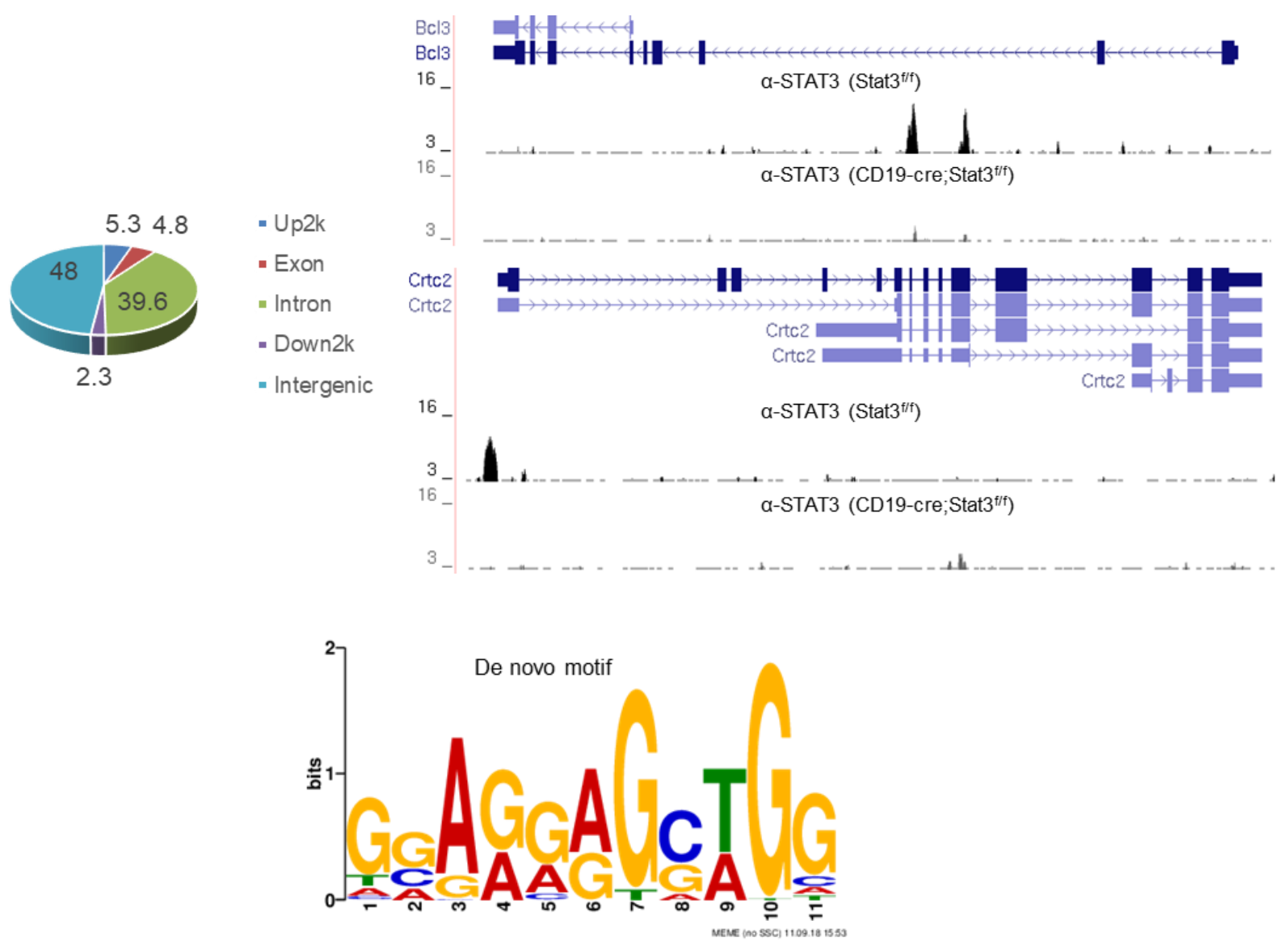

Figure 52: STAT3 binding patterns in GC B cells. In vitro cultured GC B cells from STAT3 control and cKO mice were used for ChIP-seq study. (Left) The genomic distribution (\%) of the identified STAT3-binding sites in GC B cells. (Right) Quantitative correlation of STAT3 at the $\mathrm{Bcl} 3$ and Crtc2 loci. (Bottom) De novo-derived STAT3 DNA binding motif. De novo motif was identified from the STAT3 ChIP-seq binding sites using MEME software. 

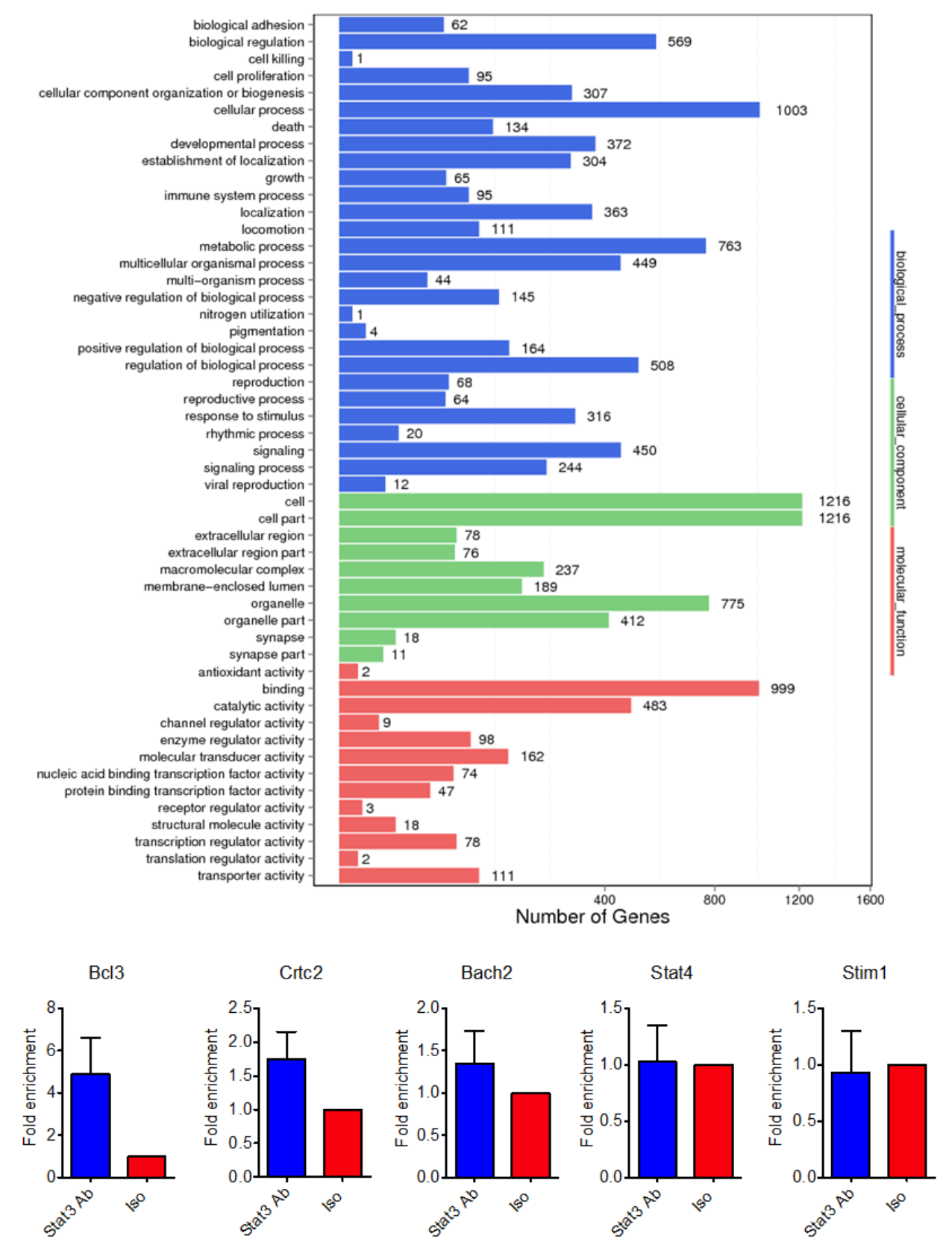

Figure 53: Gene Ontology (GO) analysis of differentially expressed peak related genes in STAT3 control and cKO GC B cells. Chromatins from wildtype GC B cells precipitated with STAT3 mAb or isotype control mAb were used for ChIP-qPCR analysis. Fold enrichment was calculated using isotype control as base level. 

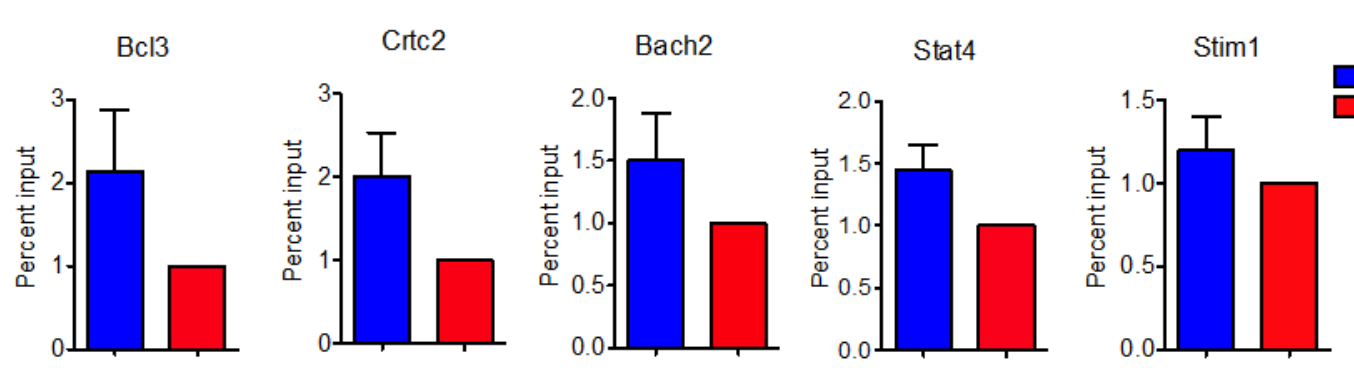

Figure 54: STAT3-bound genes. in GC B cells. Chromatins from STAT3 control and cKO mice were performed by ChIP-qPCR analysis for Bcl3, Crtc2, Bach2, Stat4, and Stim1. Percent of input was calculated using corresponding input as base level. 


\section{STAT3 in B cells regulates lung inflammation during inhaled allergy reactions}

Increased production of IgE is a well-known hallmark of allergic disease. Asthma in particular has been strongly linked with the production of IgE antibodies specific to a wide range of allergens (340). We thus tested the response of CD19-cre;STAT3 ${ }^{f l f l}$ mice in an induced asthma-like disease model. Briefly, mice were sensitized to OVA via immunization, then challenged intranasally thrice before sacrifice and analysis (Figure 55 , left).

Consistent with our previous immunization models, CD19-cre;STAT3 ${ }^{\text {flffl }}$ mice continued to display a strong phenotype of increased serum antigen-specific IgE production as compared to control mice (Figure 55, right). Immunohistochemistry staining of the lungs revealed massive infiltration of $\mathrm{Gr}-1^{+}$leukocytes, which mainly include neutrophils and eosinophils. In contrast, STAT3 proficient control mice had remarkably less infiltration, but were still increased compared to naïve mice (Figure 56). Furthermore, immunofluorescent staining of the lung tissue showed drastically more $\mathrm{CD}_{11 \mathrm{~b}^{+} \mathrm{CD} 193^{+} \text {eosinophil infiltration in CD19-cre;STAT3 }}^{\text {flflf }}$ mice (Figure 57), suggesting that STAT3 deficiency in B cells promotes allergy-induced lung inflammation. 

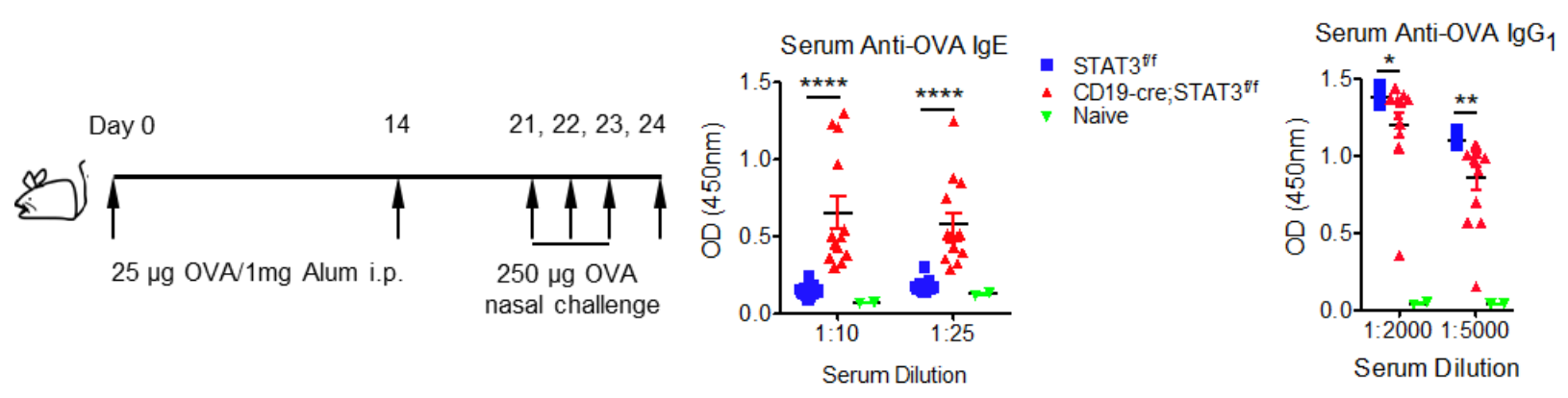

Figure 55: Generation of an asthma-like model in STAT3 deficient mice. (Left) Schema of the inhalation model. STAT3 ${ }^{\mathrm{f} / \mathrm{f}}$ control or CD19-Cre; STAT3 ${ }^{\mathrm{f} / \mathrm{f}}$ mice were immunized i.p. with $25 \mu \mathrm{g}$ OVA/1mg Alum, then boosted 14 days later. On day 21 post immunization, mice were anaesthetized and challenged with $250 \mu \mathrm{g}$ OVA intranasally, followed by repeated challenges on days 22 and 23. Mice were sacrificed on day 24 . (Right) Sera were collected at indicated time points and anti-OVA antibody levels of different classes were detected by ELISA. Data are means \pm s.e.m. Summarized data are from at least three independent experiments. ${ }^{*} P<0.05 ; * * P<0.01 ; * * * * P<0.0001$ (unpaired two-tailed Student's $t$-test) 


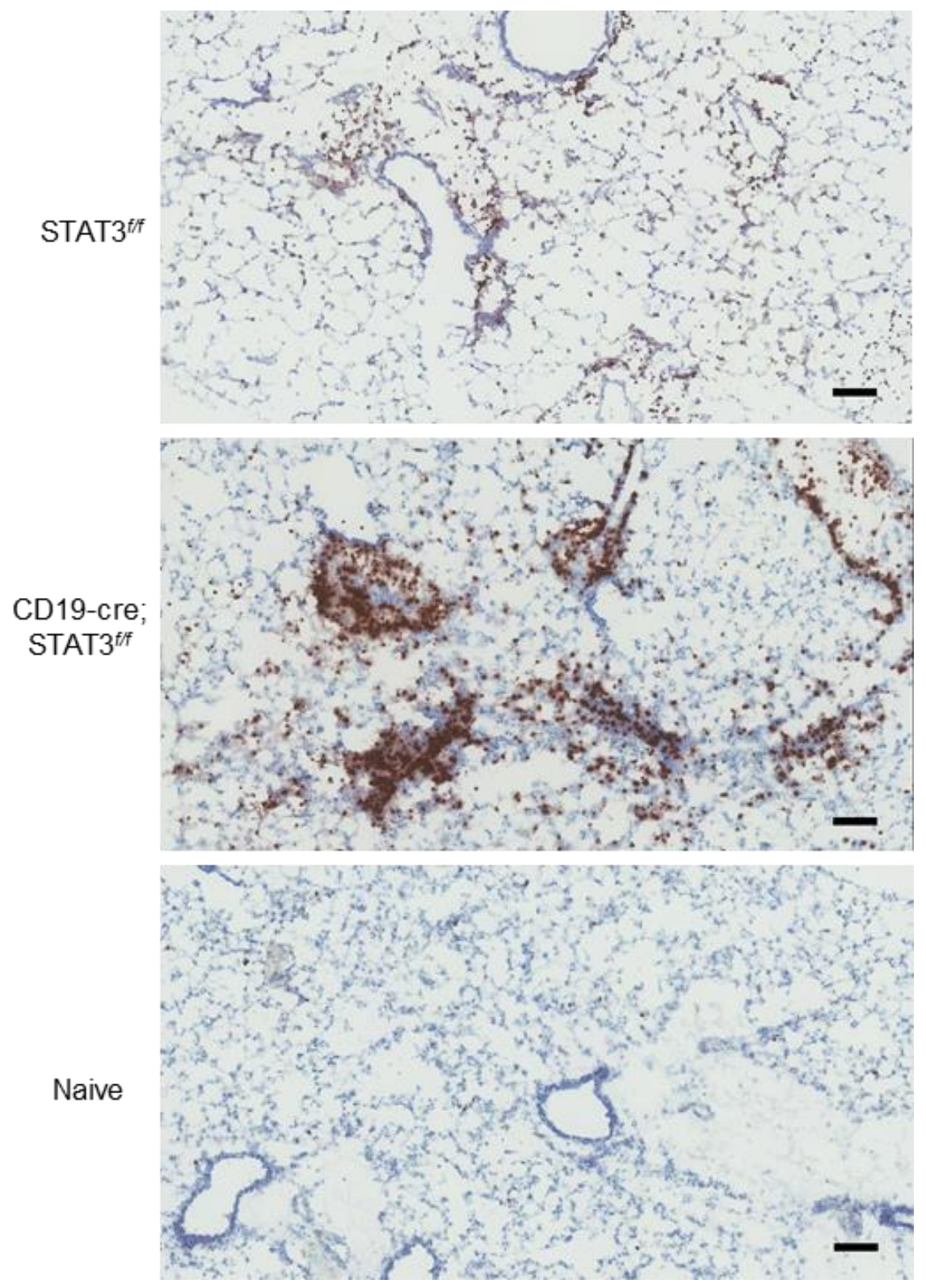

Figure 56: STAT3 deficiency causes increased inflammation in an asthma-like

model. Immunohistochemical staining of Gr-1 cells in the lungs of day 24 mice. Scale bar: $100 \mu \mathrm{m}$. Images were acquired by Aperio ScanScope digital scanner. Lung section from naïve mice was used as control. 

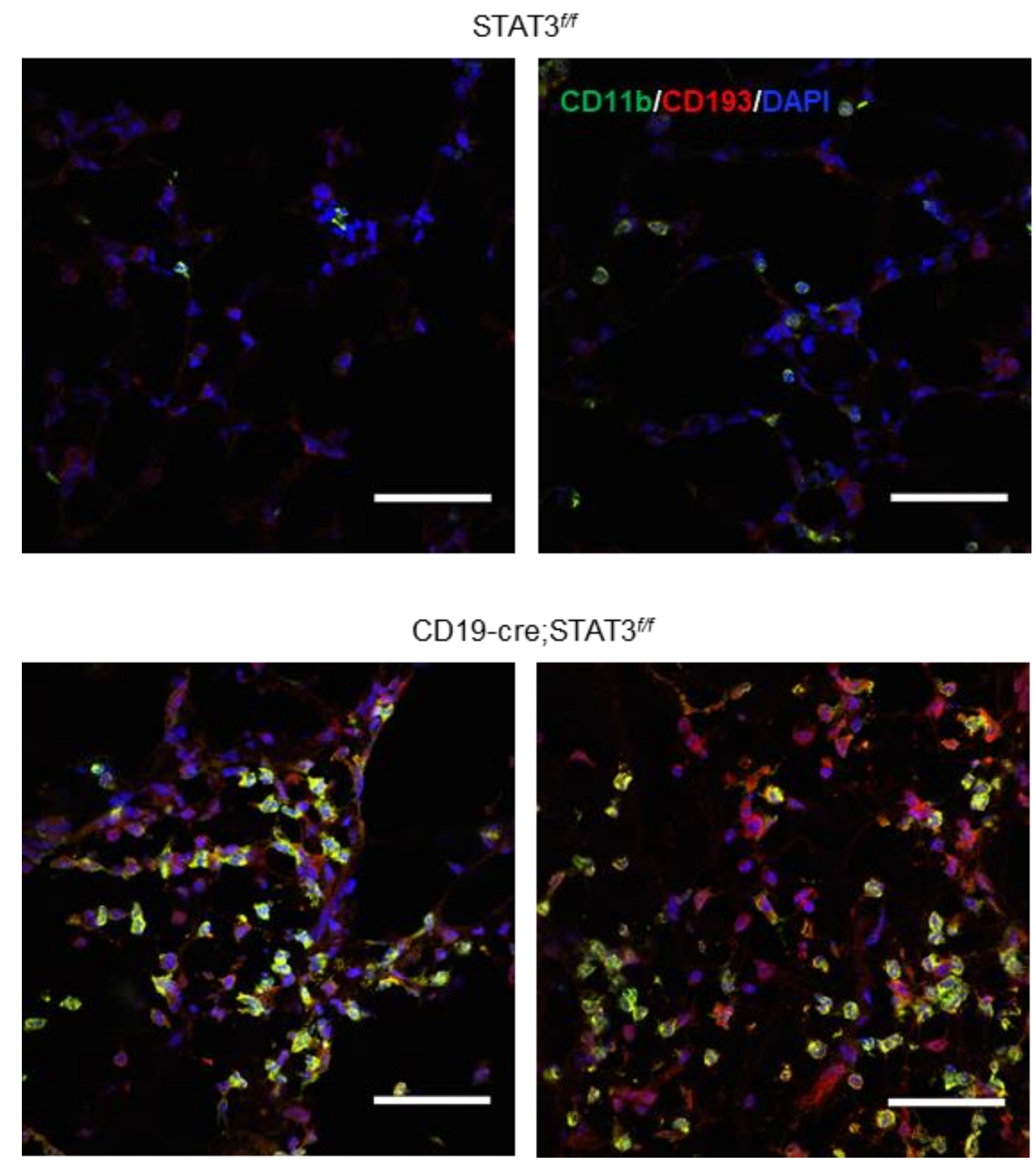

Figure 57: STAT3 deficiency causes increased eosinophil infiltration in an asthma-

like model. Immunofluorescence staining with FITC-CD11b, APC-CD193, and DAPI in the lungs of day 24 mice. Scale bar: $50 \mu \mathrm{m}$. Images were obtained using Nikon ECLIPSE Ti fluorescence microscopy. Representative images from two mice in each group are shown. 


\section{DISCUSSION}

STAT3 is a central and essential signaling molecule for many different cell types. In this study, we have shown its function in B cells as a negative regulator of IgE class switching in the GC reaction. Previous work by other groups has demonstrated STAT6 plays a critical role in the regulation of IgE germline expression in response to IL-4 upstream signaling (341). Nonfunctional mutations in STAT3 have been strongly associated with incidence of AD-HIES. However, how STAT3 dysfunction leads to increased IgE production is unclear. Our group recently found that STAT3 deficiency leads to a defect in GC formation and IgG antibody production following hRBC immunization and Th1-type immune responses in mice $(330,342)$. These data in combination with novel findings that $\mathrm{IgG}_{1} \mathrm{GC} B$ cells are able to sequentially switch to IgE led us to investigate the impact of STAT3 on this pathway $(343,344)$.

Stable interaction of $\mathrm{T}_{\mathrm{fh}}$ cells with B cells is a pillar of the GC reaction. STAT3 has already been shown to regulate Tfh differentiation and GC participation, but the function of STAT3 in GC B cells is not well studied $(345,346)$. Using a CD2-Cre driven model to knock out STAT3 in both these cell types, we revealed a defect in GC formation and $\mathrm{IgG}_{1}$ production under Th2 driven immunization, mirroring prior studies using Th1 reactions(330). However, a STAT3-knockout driven increase in production and class

switching of IgE antibody has not been previously reported, suggesting this may be a Th2 dependent phenomenon.

Despite the importance of STAT3 in $\mathrm{T}_{\mathrm{fh}}$ cell maintenance, use of a B cell specific knockout CD19-Cre model was able to replicate this phenotype, suggesting a B cell intrinsic defect. The reproduction of this phenomenon using B cell in vitro systems 
further supports this conclusion. Interestingly, the frequency of antibody-producing plasma cells was unchanged following immunization despite the decrease in frequency of GC B cells, while the IgE expressing subpopulation increased. This suggests that effect of the STAT3 defect is limited to the GC reaction while the proliferation of the end-stage plasma cell product is not significantly affected. We also showed that loss of STAT3 causes a disruption in the light zone and dark zone distribution of GC B cells. Migration between the light zone and dark zone of the GC is an essential part of the class switch and affinity maturation process, controlled by factors such as FOXO1, PI3K, and CXCR4 $(145,347,348)$. Indeed, FOXO1 expression was reduced in all STAT3 deficient GCs, though CXCR4 was unchanged in in vivo GC B cells. In other cell types, STAT3 has been shown to bind to the FOXO1 promoter and up-regulate expression, and thus may be directly controlling FOXO1 in B cells as well (349). Notably, we did demonstrate reduced IgE affinity in both CD2-Cre and CD19-Cre models, despite increased overall IgE production. This further implicates STAT3's effect on not only GC formation, but the efficacy of the antibodies it generates as well. However, it remains unclear whether the disruption in $\mathrm{IgG}_{1} / \mathrm{IgE}$ class switching and affinity maturation is a direct consequence of the light zone/dark zone distribution defect, or another unrelated symptom of STAT3 deficiency.

Class switching occurs in mature B cells in response to antigen stimulation and costimulatory signals. Excision of the DNA between the baseline mu promoter and downstream effector promoters causes expression of a new antibody class (350). $\operatorname{IgE}$ is unique in that and this process may happen either via a direct switch from IgM, or through sequential switch first to the $\mathrm{IgG}$ class followed by further switching to the 
downstream IgE promoter $(350,351)$. Our findings suggest that STAT3 suppresses this sequential switching pathway, indicated by the associative drop in $\operatorname{IgG}_{1}$ and elevated $\operatorname{IgE}$ expression. This observation would suggest STAT3 may also be dampening the effectiveness of the IgE antibody response, as sequential switching produces higher affinity antibody than direct switching (327). However, we have found reduced IgE affinity in our knockout models, indicating a more complex role for STAT3 in IgE production.

Direct regulation of both $\mathrm{IgG} 1$ and IgE class switching is reliant on Th2 cytokine signaling, most notably IL-4. STAT6 acts downstream of IL-4, binding to the I $\varepsilon$ promoter and initiating IgE expression (352). Critical GC regulator Bcl6 has binding sites within the I $\varepsilon$ promoter which overlap those of STAT6, acting as an antagonist $(353,354)$. STAT3 acts as an enhancer of Bcl6 expression and has confirmed binding sites within the Bcl6 promoter $(355,356)$. Indeed, GC Bcl6 expression was reduced in both our STAT3 deficient in vivo and in vitro models. Therefore, STAT3 may be acting as a negative regulator of IgE class switching via enhancement of STAT6 competitor Bcl6. STAT3 activation also causes increased expression of IRF4, which is required for GC formation $(357,358)$. We observed reduced IRF4 expression in knockout GCs and plasma cells. IRF4 downstream targets Aicda and IRF5 were reduced as well, which are vital in class switch regulation and $\mathrm{B}$ cell differentiation, respectively (338). These findings implicate STAT3 as a major upstream regulator of GC formation, function, and class switching. However, STAT3 ChIP-seq analysis reveals that none of these genes are identified as direct targets of STAT3. In addition, ID2 and NFIL3, two transcription factors critical for controlling IgE class switching do not have direct STAT3 binding sites, suggesting that 
STAT3 may use an unidentified factor(s) to regulate IgE isotype switching in the GC B cells $(325,359)$. It is also possible that STAT3 targets of in vitro cultured GC-like B cells are different than in vivo primary GC B cells despite their similar phenotype. Notably, Crtc2 has strong binding site on STAT3. Crtc2 is a transcriptional coactivator for CREB1 (cAMP response element). Previous studies demonstrate that Crtc2 plays an important role in plasma cell differentiation and GC B cell malignancy $(360,361)$. Interestingly, Aicda, Myc, Bach2, TCF3, and TCL1 are among 136 direct Crtc2 target genes (361). It is possible that STAT3 may regulate GC differentiation and IgE isotype switching through Crtc2.

In humans, STAT3 mutations have been strongly linked to incidence of AD-HIES, also known as Job's syndrome $(362,363)$. IgE is also known to mediate inflammatory symptoms in several allergic respiratory disorders (364). Additionally, STAT3 has been demonstrated to be involved in Th2 differentiation that may contribute to asthma disease (271). Despite high levels of IgE, AD-HIES patient incidence of allergy, anaphylaxis, and asthma condition is much less than that of other IgE related diseases (365). Inhibition of STAT3 in lung epithelial cells can even prevent lung inflammation in some asthma models, suggesting a protective role aginst allergy reaction in certain tissues (366).We demonstrated an increased eosinophil and inflammatory response in the lungs of CD19Cre driven knockout mice challenged in an asthma model, indicating B cell STAT3 is an effector of the allergic long response. However, this effect is likely ablated in many ADHIES patients by the protective loss of STAT3 in other tissues. It remains to be seen if other hyper-IgE disease hallmarks, such as susceptibility to recurrent pyogenic pneumonias, can also be caused by B cell STAT3 deficiency in mice (367). Impairment 
of the $\mathrm{IgG}$ response magnitude and affinity due to defective STAT3 are likely significant contributing factors to these frequent infections. Mice expressing a nonfunctional form of STAT3 were previously shown to be more susceptible to $C$ rodentium infection and inflammation of the colon, further supporting the role of STAT3 in protection from bacterial infection (368).

To conclude, we have shown that STAT3 is able to control IgE class switching indirectly through regulation of vital upstream factors, which can alter the inflammatory response in the lungs. These newly discovered factors may serve a potential theraupetic targets for treatment of AD-HIES patients and other IgE related diseases. 


\section{REFERENCES}

1. Medzhitov, R. 2007. Recognition of microorganisms and activation of the immune response. Nature 449: 819.

2. Parra, D., F. Takizawa, and J. O. Sunyer. 2013. Evolution of B Cell Immunity. Annual review of animal biosciences 1: 65-97.

3. Deng, L., M. Luo, A. Velikovsky, and R. A. Mariuzza. 2013. Structural Insights into the Evolution of the Adaptive Immune System. Annual Review of Biophysics 42: 191-215.

4. Sammy, B., H. W. R., and M. S. N. 2016. CD4+ T-cell help amplifies innate signals for primary CD8+ T-cell immunity. Immunological Reviews 272: 52-64.

5. Nimmerjahn, F., and J. V. Ravetch. 2008. Fc $\gamma$ receptors as regulators of immune responses. Nature Reviews Immunology 8: 34.

6. Amigorena, S., and C. Bonnerot. 1999. Fc receptors for IgG and antigen presentation on MHC class I and class II molecules. Seminars in Immunology 11: 385-390.

7. de Vallière, S., G. Abate, A. Blazevic, R. M. Heuertz, and D. F. Hoft. 2005. Enhancement of Innate and Cell-Mediated Immunity by Antimycobacterial Antibodies. Infection and Immunity 73: 6711-6720.

8. Meffre, E., and H. Wardemann. 2008. B-cell tolerance checkpoints in health and autoimmunity. Curr Opin Immunol 20: 632-638.

9. Wardemann, H., S. Yurasov, A. Schaefer, J. W. Young, E. Meffre, and M. C. Nussenzweig. 2003. Predominant autoantibody production by early human B cell precursors. Science 301: 1374-1377.

10. Pelanda, R., and R. M. Torres. 2012. Central B-Cell Tolerance: Where Selection Begins. Cold Spring Harbor Perspectives in Biology 4.

11. Aplin, B. D., C. L. Keech, A. L. de Kauwe, T. P. Gordon, D. Cavill, and J. McCluskey. 2003. Tolerance through Indifference: Autoreactive B Cells to the Nuclear Antigen La Show No Evidence of Tolerance in a Transgenic Model. The Journal of Immunology 171: 5890-5900.

12. Mandik-Nayak, L., J. Racz, B. P. Sleckman, and P. M. Allen. 2006. Autoreactive marginal zone B cells are spontaneously activated but lymph node B cells require T cell help. The Journal of Experimental Medicine 203: 1985-1998.

13. Brinkmann, M. M., E. Spooner, K. Hoebe, B. Beutler, H. L. Ploegh, and Y.-M. Kim. 2007. The interaction between the ER membrane protein UNC93B and TLR3, 7, and 9 is crucial for TLR signaling. The Journal of Cell Biology 177: 265-275.

14. Marshak-Rothstein, A. 2006. Toll-like receptors in systemic autoimmune disease. Nature Reviews Immunology 6: 823.

15. Savarese, E., C. Steinberg, R. D. Pawar, W. Reindl, S. Akira, H.-J. Anders, and A. Krug. 2008. Requirement of toll-like receptor 7 for pristane-induced production of 
autoantibodies and development of murine lupus nephritis. Arthritis \& Rheumatism 58: 1107-1115.

16. Christensen, S. R., J. Shupe, K. Nickerson, M. Kashgarian, Richard A. Flavell, and M. J. Shlomchik. 2006. Toll-like Receptor 7 and TLR9 Dictate Autoantibody Specificity and Have Opposing Inflammatory and Regulatory Roles in a Murine Model of Lupus. Immunity 25: 417-428.

17. Pisitkun, P., J. A. Deane, M. J. Difilippantonio, T. Tarasenko, A. B. Satterthwaite, and S. Bolland. 2006. Autoreactive B Cell Responses to RNA-Related Antigens Due to <em>TLR7</em> Gene Duplication. Science 312: 1669-1672.

18. Kawasaki, A., H. Furukawa, Y. Kondo, S. Ito, T. Hayashi, M. Kusaoi, I. Matsumoto, S. Tohma, Y. Takasaki, H. Hashimoto, T. Sumida, and N. Tsuchiya. 2011. TLR7 single-nucleotide polymorphisms in the 3' untranslated region and intron 2 independently contribute to systemic lupus erythematosus in Japanese women: a case-control association study. Arthritis Research \& Therapy 13: R41R41.

19. Sjöstrand, M., A. Johansson, L. Aqrawi, T. Olsson, M. Wahren-Herlenius, and A. Espinosa. 2016. The Expression of BAFF Is Controlled by IRF Transcription Factors. The Journal of Immunology 196: 91-96.

20. Crow, M. K. 2014. Type I Interferon in the Pathogenesis of Lupus. Journal of immunology (Baltimore, Md. : 1950) 192: 5459-5468.

21. Castillejo-López, C., A. M. Delgado-Vega, J. Wojcik, S. V. Kozyrev, E. Thavathiru, Y.-Y. Wu, E. Sánchez, D. Pöllmann, J. R. López-Egido, S. Fineschi, N. Domínguez, R. Lu, J. A. James, J. T. Merrill, J. A. Kelly, K. M. Kaufman, K. L. Moser, G. Gilkeson, J. Frostegård, B. A. Pons-Estel, S. D'Alfonso, T. Witte, J. L. Callejas, J. B. Harley, P. M. Gaffney, J. Martin, J. M. Guthridge, and M. E. Alarcón-Riquelme. 2012. Genetic and physical interaction of the B-cell systemic lupus erythematosus-associated genes <em>BANK $1</ \mathrm{em}>$ and $<\mathrm{em}>\mathrm{BLK}</ \mathrm{em}>$. Annals of the Rheumatic Diseases 71: 136-142.

22. Vaughn, S. E., L. C. Kottyan, M. E. Munroe, and J. B. Harley. 2012. Genetic susceptibility to lupus: the biological basis of genetic risk found in B cell signaling pathways. Journal of Leukocyte Biology 92: 577-591.

23. Wu, Y.-Y., R. Kumar, R. Iida, H. Bagavant, and M. E. Alarcón-Riquelme. 2016. BANK1 Regulates IgG Production in a Lupus Model by Controlling TLR7Dependent STAT1 Activation. PLOS ONE 11: e0156302.

24. Coughlin, J. J., S. L. Stang, N. A. Dower, and J. C. Stone. 2005. RasGRP1 and RasGRP3 Regulate B Cell Proliferation by Facilitating B Cell Receptor-Ras Signaling. The Journal of Immunology 175: 7179-7184.

25. Liu, L., D. Gritz, C. A. Parent, and D. Montell. 2014. PKC $\beta I I$ acts downstream of chemoattractant receptors and mTORC2 to regulate cAMP production and myosin II activity in neutrophils. Molecular Biology of the Cell 25: 1446-1457.

26. Lamagna, C., Y. Hu, A. L. DeFranco, and C. A. Lowell. 2014. B Cell-Specific Loss of Lyn Kinase Leads to Autoimmunity. The Journal of Immunology 192: 919-928.

27. Hua, Z., A. J. Gross, C. Lamagna, N. Ramos-Hernández, P. Scapini, M. Ji, H. Shao, C. A. Lowell, B. Hou, and A. L. DeFranco. 2014. Requirement for MyD88 Signaling in B Cells and Dendritic Cells for Germinal Center Anti-Nuclear 
Antibody Production in Lyn-Deficient Mice. The Journal of Immunology 192: 875-885.

28. Samuelson, E. M., R. M. Laird, A. C. Maue, R. Rochford, and S. M. Hayes. 2012. Blk haploinsufficiency impairs the development, but enhances the functional responses, of MZ B cells. Immunology and Cell Biology 90: 620-629.

29. Samuelson, E. M., R. M. Laird, A. M. Papillion, A. H. Tatum, M. F. Princiotta, and S. M. Hayes. 2014. Reduced B Lymphoid Kinase (Blk) Expression Enhances Proinflammatory Cytokine Production and Induces Nephrosis in C57BL/6-lpr/lpr Mice. PLOS ONE 9: e92054.

30. Manjarrez-Orduño, N., E. Marasco, S. A. Chung, M. S. Katz, J. F. Kiridly, K. R. Simpfendorfer, J. Freudenberg, D. H. Ballard, E. Nashi, T. J. Hopkins, D. S.

Cunninghame Graham, A. T. Lee, M. J. H. Coenen, B. Franke, D. W. Swinkels, R. R. Graham, R. P. Kimberly, P. M. Gaffney, T. J. Vyse, T. W. Behrens, L. A. Criswell, B. Diamond, and P. K. Gregersen. 2012. CSK regulatory polymorphism is associated with systemic lupus erythematosus and influences B-cell signaling and activation. Nature Genetics 44: 1227.

31. Hata, A., H. Sabe, T. Kurosaki, M. Takata, and H. Hanafusa. 1994. Functional analysis of Csk in signal transduction through the B-cell antigen receptor. Molecular and Cellular Biology 14: 7306-7313.

32. Bottini, N., L. Musumeci, A. Alonso, S. Rahmouni, K. Nika, M. Rostamkhani, J. MacMurray, G. F. Meloni, P. Lucarelli, M. Pellecchia, G. S. Eisenbarth, D. Comings, and T. Mustelin. 2004. A functional variant of lymphoid tyrosine phosphatase is associated with type I diabetes. Nature Genetics 36: 337.

33. Bottini, N., T. Vang, F. Cucca, and T. Mustelin. 2006. Role of PTPN22 in type 1 diabetes and other autoimmune diseases. Seminars in Immunology 18: 207-213.

34. Oleksyn, D., M. Pulvino, J. Zhao, R. Misra, A. Vosoughi, S. Jenks, C. Tipton, F. Lund, G. Schwartz, B. Goldman, C. Mohan, K. Mehta, M. Mehta, M. Leitgets, I. Sanz, and L. Chen. 2013. Protein Kinase C $\beta$ Is Required for Lupus Development in Sle Mice. Arthritis \& Rheumatism 65: 1022-1031.

35. Getahun, A., N. A. Beavers, S. R. Larson, M. J. Shlomchik, and J. C. Cambier. 2016. Continuous inhibitory signaling by both SHP-1 and SHIP-1 pathways is required to maintain unresponsiveness of anergic B cells. The Journal of Experimental Medicine 213: 751-769.

36. Maxwell, M. J., M. Duan, J. E. Armes, G. P. Anderson, D. M. Tarlinton, and M. L. Hibbs. 2011. Genetic Segregation of Inflammatory Lung Disease and Autoimmune Disease Severity in SHIP-1<sup $>-1-</$ sup $>$ Mice. The Journal of Immunology 186: 7164-7175.

37. Pao, L. I., K.-P. Lam, J. M. Henderson, J. L. Kutok, M. Alimzhanov, L. Nitschke, M. L. Thomas, B. G. Neel, and K. Rajewsky. 2007. B Cell-Specific Deletion of Protein-Tyrosine Phosphatase Shp1 Promotes B-1a Cell Development and Causes Systemic Autoimmunity. Immunity 27: 35-48.

38. Adachi, T., J. Wienands, C. Wakabayashi, H. Yakura, M. Reth, and T. Tsubata. 2001. SHP-1 Requires Inhibitory Co-receptors to Down-modulate B Cell Antigen Receptor-mediated Phosphorylation of Cellular Substrates. Journal of Biological Chemistry 276: 26648-26655. 
39. Hitomi, Y., N. Tsuchiya, A. Kawasaki, J. Ohashi, T. Suzuki, C. Kyogoku, T. Fukazawa, S. Bejrachandra, U. Siriboonrit, D. Chandanayingyong, P.

Suthipinittharm, B. P. Tsao, H. Hashimoto, Z. Honda, and K. Tokunaga. 2004. CD72 polymorphisms associated with alternative splicing modify susceptibility to human systemic lupus erythematosus through epistatic interaction with FCGR2B. Hum Mol Genet 13: 2907-2917.

40. Akatsu, C., K. Shinagawa, N. Numoto, Z. Liu, A. K. Ucar, M. Aslam, S. Phoon, T. Adachi, K. Furukawa, N. Ito, and T. Tsubata. 2016. CD72 negatively regulates B lymphocyte responses to the lupus-related endogenous toll-like receptor 7 ligand Sm/RNP. The Journal of Experimental Medicine 213: 2691-2706.

41. Li, D. H., J. W. Tung, I. H. Tarner, A. L. Snow, T. Yukinari, R.

Ngernmaneepothong, O. M. Martinez, and J. R. Parnes. 2006. CD72 downmodulates BCR-induced signal transduction and diminishes survival in primary mature B lymphocytes. J Immunol 176: 5321-5328.

42. Jellusova, J., U. Wellmann, K. Amann, T. H. Winkler, and L. Nitschke. 2010. CD22 × Siglec-G Double-Deficient Mice Have Massively Increased B1 Cell Numbers and Develop Systemic Autoimmunity. The Journal of Immunology 184: 3618-3627.

43. Xu, M., R. Hou, A. Sato-Hayashizaki, R. Man, C. Zhu, C. Wakabayashi, S. Hirose, T. Adachi, and T. Tsubata. 2013. $<\mathrm{em}>\mathrm{Cd} 72<$ sup $>\mathrm{c}</$ sup $></$ em $>$ Is a Modifier Gene that Regulates <em $>$ Fas $<$ sup $>$ lpr $</$ sup $></$ em $>$-Induced Autoimmune Disease. The Journal of Immunology 190: 5436-5445.

44. Baumgarth, N. 2010. The double life of a B-1 cell: self-reactivity selects for protective effector functions. Nature Reviews Immunology 11: 34.

45. Sindhava, V. J., and S. Bondada. 2012. Multiple Regulatory Mechanisms Control B-1 B Cell Activation. Frontiers in Immunology 3: 372.

46. Alugupalli, K. R., J. M. Leong, R. T. Woodland, M. Muramatsu, T. Honjo, and R. M. Gerstein. 2004. B1b Lymphocytes Confer T Cell-Independent Long-Lasting Immunity. Immunity 21: 379-390.

47. Berland, R., and H. H. Wortis. 2002. Origins and Functions of B-1 Cells with Notes on the Role of CD5. Annual Review of Immunology 20: 253-300.

48. Hoffmann, A., S. Kerr, J. Jellusova, J. Zhang, F. Weisel, U. Wellmann, T. H. Winkler, B. Kneitz, P. R. Crocker, and L. Nitschke. 2007. Siglec-G is a B1 cellinhibitory receptor that controls expansion and calcium signaling of the B1 cell population. Nature Immunology 8: 695.

49. Carter, N. A., R. Vasconcellos, E. C. Rosser, C. Tulone, A. Muñoz-Suano, M. Kamanaka, M. R. Ehrenstein, R. A. Flavell, and C. Mauri. 2011. Mice Lacking Endogenous IL-10-Producing Regulatory B Cells Develop Exacerbated Disease and Present with an Increased Frequency of Th1/Th17 but a Decrease in Regulatory T Cells. The Journal of Immunology 186: 5569-5579.

50. Ronet, C., Y. H.-L. Torre, M. Revaz-Breton, B. Mastelic, F. Tacchini-Cottier, J. Louis, and P. Launois. 2010. Regulatory B Cells Shape the Development of Th2 Immune Responses in BALB/c Mice Infected with <em>Leishmania major</em> Through IL-10 Production. The Journal of Immunology 184: 886-894.

51. Maseda, D., S. H. Smith, D. J. DiLillo, J. M. Bryant, K. M. Candando, C. T. Weaver, and T. F. Tedder. 2012. Regulatory B10 Cells Differentiate into 
Antibody-Secreting Cells After Transient IL-10 Production In Vivo. The Journal of Immunology 188: 1036-1048.

52. Mohan, C., S. Adams, V. Stanik, and S. K. Datta. 1993. Nucleosome: a major immunogen for pathogenic autoantibody-inducing T cells of lupus. The Journal of Experimental Medicine 177: 1367-1381.

53. Bobbitt, K. R., and L. B. Justement. 2000. Regulation of MHC Class II Signal Transduction by the B Cell Coreceptors CD19 and CD22. The Journal of Immunology 165: 5588-5596.

54. Graham, R. R., W. Ortmann, P. Rodine, K. Espe, C. Langefeld, E. Lange, A. Williams, S. Beck, C. Kyogoku, K. Moser, P. Gaffney, P. K. Gregersen, L. A. Criswell, J. B. Harley, and T. W. Behrens. 2007. Specific combinations of HLADR2 and DR3 class II haplotypes contribute graded risk for disease susceptibility and autoantibodies in human SLE. European Journal Of Human Genetics 15: 823.

55. Manku, H., D. S. C. Graham, and T. J. Vyse. 2009. Association of the costimulator OX40L with systemic lupus erythematosus. Journal of Molecular Medicine 87: 229-234.

56. Martin, F., A. M. Oliver, and J. F. Kearney. 2001. Marginal Zone and B1 B Cells Unite in the Early Response against T-Independent Blood-Borne Particulate Antigens. Immunity 14: 617-629.

57. Puga, I., M. Cols, C. M. Barra, B. He, L. Cassis, M. Gentile, L. Comerma, A. Chorny, M. Shan, W. Xu, G. Magri, D. M. Knowles, W. Tam, A. Chiu, J. B. Bussel, S. Serrano, J. A. Lorente, B. Bellosillo, J. Lloreta, N. Juanpere, F. Alameda, T. Baró, C. D. de Heredia, N. Torán, A. Català, M. Torrebadell, C. Fortuny, V. Cusi, C. Carreras, G. A. Diaz, J. M. Blander, C.-M. Farber, G. Silvestri, C. Cunningham-Rundles, M. Calvillo, C. Dufour, L. D. Notarangelo, V. Lougaris, A. Plebani, J.-L. Casanova, S. C. Ganal, A. Diefenbach, J. I. Aróstegui, M. Juan, J. Yagüe, N. Mahlaoui, J. Donadieu, K. Chen, and A. Cerutti. 2012. Bhelper neutrophils stimulate immunoglobulin diversification and production in the marginal zone of the spleen. Nature immunology 13: 170-180.

58. Steiniger, B., E. M. Timphus, and P. J. Barth. 2006. The splenic marginal zone in humans and rodents: an enigmatic compartment and its inhabitants. Histochemistry and Cell Biology 126: 641-648.

59. Zeng, D., M.-K. Lee, J. Tung, A. Brendolan, and S. Strober. 2000. Cutting Edge: A Role for CD1 in the Pathogenesis of Lupus in NZB/NZW Mice. The Journal of Immunology 164: 5000-5004.

60. Sang, A., Y.-Y. Zheng, and L. Morel. 2014. Contributions of B cells to lupus pathogenesis. Molecular immunology 62: 329-338.

61. van den Eertwegh, A. J. M., J. D. Laman, M. M. Schellekens, W. J. A. Boersma, and E. Claassen. 1992. Complement-mediated follicular localization of Tindependent type-2 antigens: the role of marginal zone macrophages revisited. European Journal of Immunology 22: 719-726.

62. Toellner, K.-M., S. A. Luther, D. M. Y. Sze, R. K. W. Choy, D. R. Taylor, I. C. M. MacLennan, and H. Acha-Orbea. 1998. T Helper 1 (Th1) and Th2 Characteristics Start to Develop During T Cell Priming and Are Associated with 
an Immediate Ability to Induce Immunoglobulin Class Switching. The Journal of Experimental Medicine 187: 1193-1204.

63. Chan, T. D., D. Gatto, K. Wood, T. Camidge, A. Basten, and R. Brink. 2009. Antigen Affinity Controls Rapid T-Dependent Antibody Production by Driving the Expansion Rather than the Differentiation or Extrafollicular Migration of Early Plasmablasts. The Journal of Immunology 183: 3139-3149.

64. MacLennan, I. C. M., K.-M. Toellner, A. F. Cunningham, K. Serre, D. M.-Y. Sze, E. Zúñiga, M. C. Cook, and C. G. Vinuesa. 2003. Extrafollicular antibody responses. Immunological Reviews 194: 8-18.

65. García de Vinuesa, C., A. Gulbranson-Judge, M. Khan, P. O'Leary, M. Cascalho, M. Wabl, G. G. B. Klaus, M. J. Owen, and I. C. M. MacLennan. 1999. Dendritic cells associated with plasmablast survival. European Journal of Immunology 29: 3712-3721.

66. Lee, S. K., R. J. Rigby, D. Zotos, L. M. Tsai, S. Kawamoto, J. L. Marshall, R. R. Ramiscal, T. D. Chan, D. Gatto, R. Brink, D. Yu, S. Fagarasan, D. M. Tarlinton, A. F. Cunningham, and C. G. Vinuesa. 2011. B cell priming for extrafollicular antibody responses requires Bcl-6 expression by T cells. The Journal of Experimental Medicine 208: 1377-1388.

67. Blink, E. J., A. Light, A. Kallies, S. L. Nutt, P. D. Hodgkin, and D. M. Tarlinton. 2005. Early appearance of germinal center-derived memory B cells and plasma cells in blood after primary immunization. The Journal of Experimental Medicine 201: 545-554.

68. Chernova, I., D. D. Jones, J. R. Wilmore, A. Bortnick, M. Yucel, U. Hershberg, and D. Allman. 2014. Lasting antibody responses are mediated by a combination of newly formed and established bone marrow plasma cells drawn from clonally distinct precursors. Journal of immunology (Baltimore, Md. : 1950) 193: 49714979.

69. Liu, Z., Y. Zou, and A. Davidson. 2011. Plasma cells in systemic lupus erythematosus: the long and short of it all. Eur J Immunol 41: 588-591.

70. Malkiel, S., A. N. Barlev, Y. Atisha-Fregoso, J. Suurmond, and B. Diamond. 2018. Plasma Cell Differentiation Pathways in Systemic Lupus Erythematosus. Frontiers in Immunology 9: 427.

71. Deng, R., C. Hurtz, Q. Song, C. Yue, G. Xiao, H. Yu, X. Wu, M. Muschen, S. Forman, P. J. Martin, and D. Zeng. 2017. Extrafollicular CD4(+) T-B interactions are sufficient for inducing autoimmune-like chronic graft-versus-host disease. Nat Commun 8: 978.

72. Kitano, M., S. Moriyama, Y. Ando, M. Hikida, Y. Mori, T. Kurosaki, and T. Okada. 2011. Bcl6 Protein Expression Shapes Pre-Germinal Center B Cell Dynamics and Follicular Helper T Cell Heterogeneity. Immunity 34: 961-972.

73. Coffey, F., B. Alabyev, and T. Manser. 2009. Initial clonal expansion of germinal center B cells takes place at the perimeter of follicles. Immunity 30: 599-609.

74. Okada, T., M. J. Miller, I. Parker, M. F. Krummel, M. Neighbors, S. B. Hartley, A. O'Garra, M. D. Cahalan, and J. G. Cyster. 2005. Antigen-Engaged B Cells Undergo Chemotaxis toward the T Zone and Form Motile Conjugates with Helper T Cells. PLoS Biology 3: e150. 
75. Garin, A., M. Meyer-Hermann, M. Contie, M. T. Figge, V. Buatois, M. Gunzer, K.-M. Toellner, G. Elson, and M. H. Kosco-Vilbois. 2010. Toll-like Receptor 4 Signaling by Follicular Dendritic Cells Is Pivotal for Germinal Center Onset and Affinity Maturation. Immunity 33: 84-95.

76. Heesters, B. A., R. C. Myers, and M. C. Carroll. 2014. Follicular dendritic cells: dynamic antigen libraries. Nature Reviews Immunology 14: 495.

77. Luzina, I. G., S. P. Atamas, C. E. Storrer, L. C. daSilva, G. Kelsoe, J. C. Papadimitriou, and B. S. Handwerger. 2001. Spontaneous formation of germinal centers in autoimmune mice. Journal of Leukocyte Biology 70: 578-584.

78. Victora, G. D., T. A. Schwickert, D. R. Fooksman, A. O. Kamphorst, M. MeyerHermann, M. L. Dustin, and M. C. Nussenzweig. 2010. Germinal Center Dynamics Revealed by Multiphoton Microscopy Using a Photoactivatable Fluorescent Reporter. Cell 143: 592-605.

79. McHeyzer-Williams, L. J., P. J. Milpied, S. L. Okitsu, and M. G. McHeyzerWilliams. 2015. Switched-memory B cells remodel B cell receptors within secondary germinal centers. Nature immunology 16: 296-305.

80. Victora, G. D., D. Dominguez-Sola, A. B. Holmes, S. Deroubaix, R. DallaFavera, and M. C. Nussenzweig. 2012. Identification of human germinal center light and dark zone cells and their relationship to human B-cell lymphomas. Blood 120: 2240-2248.

81. Gitlin, A. D., Z. Shulman, and M. C. Nussenzweig. 2014. Clonal selection in the germinal center by regulated proliferation and hypermutation. Nature 509: 637640.

82. Allen, C. D. C., T. Okada, and J. G. Cyster. 2007. Germinal Center Organization and Cellular Dynamics. Immunity 27: 190-202.

83. Schwickert, T. A., R. L. Lindquist, G. Shakhar, G. Livshits, D. Skokos, M. H. Kosco-Vilbois, M. L. Dustin, and M. C. Nussenzweig. 2007. In vivo imaging of germinal centres reveals a dynamic open structure. Nature 446: 83.

84. Suzuki, K., I. Grigorova, T. G. Phan, L. M. Kelly, and J. G. Cyster. 2009. Visualizing B cell capture of cognate antigen from follicular dendritic cells. The Journal of Experimental Medicine 206: 1485-1493.

85. Yoon, S.-O., X. Zhang, P. Berner, B. Blom, and Y. S. Choi. 2009. Notch Ligands Expressed by Follicular Dendritic Cells Protect Germinal Center B Cells from Apoptosis. The Journal of Immunology 183: 352-358.

86. Baumann, I., W. Kolowos, R. E. Voll, B. Manger, U. Gaipl, W. L. Neuhuber, T. Kirchner, J. R. Kalden, and M. Herrmann. 2002. Impaired uptake of apoptotic cells into tingible body macrophages in germinal centers of patients with systemic lupus erythematosus. Arthritis \& Rheumatism 46: 191-201.

87. Kojima, M., S. Nakamura, Y. Morishita, H. Itoh, K. Yoshida, Y. Ohno, T. Oyama, S. Asano, T. Joshita, S. Mori, T. Suchi, and N. Masawa. 2000. Reactive follicular hyperplasia in the lymph node lesions from systemic lupus erythematosus patients: A clinicopathological and immunohistological study of 21 cases. Pathology International 50: 304-312.

88. Victoratos, P., and G. Kollias. 2009. Induction of Autoantibody-Mediated Spontaneous Arthritis Critically Depends on Follicular Dendritic Cells. Immunity 30: 130-142. 
89. Hams, E., M. J. McCarron, S. Amu, H. Yagita, M. Azuma, L. Chen, and P. G. Fallon. 2011. Blockade of B7-H1 (Programmed Death Ligand 1) Enhances Humoral Immunity by Positively Regulating the Generation of T Follicular Helper Cells. The Journal of Immunology 186: 5648-5655.

90. Good-Jacobson, K. L., C. G. Szumilas, L. Chen, A. H. Sharpe, M. M. Tomayko, and M. J. Shlomchik. 2010. PD-1 regulates germinal center B cell survival and the formation and affinity of long-lived plasma cells. Nature Immunology 11: 535.

91. Crotty, S. 2011. Follicular Helper CD4 T Cells (TFH). Annual Review of Immunology 29: 621-663.

92. Avery, D. T., V. L. Bryant, C. S. Ma, R. de Waal Malefyt, and S. G. Tangye. 2008. IL-21-Induced Isotype Switching to IgG and IgA by Human Naive B Cells Is Differentially Regulated by IL-4. The Journal of Immunology 181: 1767-1779.

93. Hao, Z., G. S. Duncan, J. Seagal, Y.-W. Su, C. Hong, J. Haight, N.-J. Chen, A. Elia, A. Wakeham, W. Y. Li, J. Liepa, G. A. Wood, S. Casola, K. Rajewsky, and T. W. Mak. 2008. Fas Receptor Expression in Germinal-Center B Cells Is Essential for T and B Lymphocyte Homeostasis. Immunity 29: 615-627.

94. Cubas, R. A., J. C. Mudd, A.-L. Savoye, M. Perreau, J. van Grevenynghe, T. Metcalf, E. Connick, A. Meditz, G. J. Freeman, G. Abesada-Terk Jr, J. M. Jacobson, A. D. Brooks, S. Crotty, J. D. Estes, G. Pantaleo, M. M. Lederman, and E. K. Haddad. 2013. Inadequate T follicular cell help impairs B cell immunity during HIV infection. Nature Medicine 19: 494.

95. He, J., Louis M. Tsai, Yew A. Leong, X. Hu, Cindy S. Ma, N. Chevalier, X. Sun, K. Vandenberg, S. Rockman, Y. Ding, L. Zhu, W. Wei, C. Wang, A. Karnowski, Gabrielle T. Belz, Joanna R. Ghali, Matthew C. Cook, D. S. Riminton, A. Veillette, Pamela L. Schwartzberg, F. Mackay, R. Brink, Stuart G. Tangye, Carola G. Vinuesa, Charles R. Mackay, Z. Li, and D. Yu. 2013. Circulating Precursor CCR7loPD-1hi CXCR5+ CD4+ T Cells Indicate Tfh Cell Activity and Promote Antibody Responses upon Antigen Reexposure. Immunity 39: 770-781.

96. Liarski, V. M., N. Kaverina, A. Chang, D. Brandt, D. Yanez, L. Talasnik, G. Carlesso, R. Herbst, T. O. Utset, C. Labno, Y. Peng, Y. Jiang, M. L. Giger, and M. R. Clark. 2014. Cell Distance Mapping Identifies Functional T Follicular Helper Cells in Inflamed Human Renal Tissue. Science Translational Medicine 6: 230ra246-230ra246.

97. Shinnakasu, R., T. Inoue, K. Kometani, S. Moriyama, Y. Adachi, M. Nakayama, Y. Takahashi, H. Fukuyama, T. Okada, and T. Kurosaki. 2016. Regulated selection of germinal-center cells into the memory B cell compartment. Nature Immunology 17: 861.

98. Weisel, Florian J., Griselda V. Zuccarino-Catania, M. Chikina, and Mark J. Shlomchik. 2016. A Temporal Switch in the Germinal Center Determines Differential Output of Memory B and Plasma Cells. Immunity 44: 116-130.

99. Zotos, D., J. M. Coquet, Y. Zhang, A. Light, K. D'Costa, A. Kallies, L. M. Corcoran, D. I. Godfrey, K.-M. Toellner, M. J. Smyth, S. L. Nutt, and D. M. Tarlinton. 2010. IL-21 regulates germinal center B cell differentiation and proliferation through a B cell-intrinsic mechanism. The Journal of Experimental Medicine 207: 365-378. 
100. Suan, D., C. Sundling, and R. Brink. 2017. Plasma cell and memory B cell differentiation from the germinal center. Current Opinion in Immunology 45: 97102.

101. Reimold, A. M., N. N. Iwakoshi, J. Manis, P. Vallabhajosyula, E. SzomolanyiTsuda, E. M. Gravallese, D. Friend, M. J. Grusby, F. Alt, and L. H. Glimcher. 2001. Plasma cell differentiation requires the transcription factor XBP-1. Nature 412: 300 .

102. Meyer-Hermann, M., E. Mohr, N. Pelletier, Y. Zhang, Gabriel D. Victora, and K.M. Toellner. 2012. A Theory of Germinal Center B Cell Selection, Division, and Exit. Cell Reports 2: 162-174.

103. Vale, A. M., and H. W. Schroeder, Jr. 2010. Clinical consequences of defects in B-cell development. The Journal of allergy and clinical immunology 125: 778787.

104. Slifka, M. K., R. Antia, J. K. Whitmire, and R. Ahmed. 1998. Humoral Immunity Due to Long-Lived Plasma Cells. Immunity 8: 363-372.

105. Hammarlund, E., A. Thomas, I. J. Amanna, L. A. Holden, O. D. Slayden, B. Park, L. Gao, and M. K. Slifka. 2017. Plasma cell survival in the absence of B cell memory. Nat Commun 8: 1781.

106. Manz, R. A., A. Thiel, and A. Radbruch. 1997. Lifetime of plasma cells in the bone marrow. Nature 388: 133.

107. Espeli, M., S. Bökers, G. Giannico, H. A. Dickinson, V. Bardsley, A. B. Fogo, and K. G. C. Smith. 2011. Local Renal Autoantibody Production in Lupus Nephritis. Journal of the American Society of Nephrology 22: 296-305.

108. Taddeo, A., L. Khodadadi, C. Voigt, I. M. Mumtaz, Q. Cheng, K. Moser, T. Alexander, R. A. Manz, A. Radbruch, F. Hiepe, and B. F. Hoyer. 2015. Longlived plasma cells are early and constantly generated in New Zealand Black/New Zealand White F1 mice and their therapeutic depletion requires a combined targeting of autoreactive plasma cells and their precursors. Arthritis Research \& Therapy 17: 39.

109. Benson, M. J., S. R. Dillon, E. Castigli, R. S. Geha, S. Xu, K.-P. Lam, and R. J. Noelle. 2008. Cutting Edge: The Dependence of Plasma Cells and Independence of Memory B Cells on BAFF and APRIL. The Journal of Immunology 180: 36553659 .

110. Rozanski, C. H., R. Arens, L. M. Carlson, J. Nair, L. H. Boise, A. A. ChananKhan, S. P. Schoenberger, and K. P. Lee. 2011. Sustained antibody responses depend on CD28 function in bone marrow-resident plasma cells. The Journal of Experimental Medicine 208: 1435-1446.

111. Cassese, G., S. Arce, A. E. Hauser, K. Lehnert, B. Moewes, M. Mostarac, G. Muehlinghaus, M. Szyska, A. Radbruch, and R. A. Manz. 2003. Plasma Cell Survival Is Mediated by Synergistic Effects of Cytokines and AdhesionDependent Signals. The Journal of Immunology 171: 1684-1690.

112. Ripley, B., B. Goncalves, D. Isenberg, D. Latchman, and A. Rahman. 2005. Raised levels of interleukin 6 in systemic lupus erythematosus correlate with anaemia. Annals of the Rheumatic Diseases 64: 849-853.

113. Salazar-Camarena, D. C., P. C. Ortiz-Lazareno, A. Cruz, E. Oregon-Romero, J. R. Machado-Contreras, J. F. Muñoz-Valle, M. Orozco-López, M. Marín-Rosales, 
and C. A. Palafox-Sánchez. 2015. Association of BAFF, APRIL serum levels, BAFF-R, TACI and BCMA expression on peripheral B-cell subsets with clinical manifestations in systemic lupus erythematosus. Lupus 25: 582-592.

114. Hoyer, B. F., K. Moser, A. E. Hauser, A. Peddinghaus, C. Voigt, D. Eilat, A. Radbruch, F. Hiepe, and R. A. Manz. 2004. Short-lived Plasmablasts and Longlived Plasma Cells Contribute to Chronic Humoral Autoimmunity in NZB/W Mice. The Journal of Experimental Medicine 199: 1577-1584.

115. Mannoor, K., A. Matejuk, Y. Xu, M. Beardall, and C. Chen. 2012. Expression of Natural Autoantibodies in MRL-lpr Mice Protects from Lupus Nephritis and Improves Survival. The Journal of Immunology 188: 3628-3638.

116. Roth, K., L. Oehme, S. Zehentmeier, Y. Zhang, R. Niesner, and A. E. Hauser. 2014. Tracking plasma cell differentiation and survival. Cytometry Part A 85: 1524.

117. Pelletier, N., L. J. McHeyzer-Williams, K. A. Wong, E. Urich, N. Fazilleau, and M. G. McHeyzer-Williams. 2010. Plasma cells negatively regulate the follicular helper T cell program. Nature immunology 11: 1110-1118.

118. Deng, Y., and B. P. Tsao. 2014. Advances in lupus genetics and epigenetics. Current opinion in rheumatology 26: 482-492.

119. The International Consortium for Systemic Lupus Erythematosus, G., J. B. Harley, M. E. Alarcón-Riquelme, L. A. Criswell, C. O. Jacob, R. P. Kimberly, K. L. Moser, B. P. Tsao, T. J. Vyse, C. D. Langefeld, S. K. Nath, J. M. Guthridge, B. L. Cobb, D. B. Mirel, M. C. Marion, A. H. Williams, J. Divers, W. Wang, S. G. Frank, B. Namjou, S. B. Gabriel, A. T. Lee, P. K. Gregersen, T. W. Behrens, K. E. Taylor, M. Fernando, R. Zidovetzki, P. M. Gaffney, J. C. Edberg, J. D. Rioux, J. O. Ojwang, J. A. James, J. T. Merrill, G. S. Gilkeson, M. F. Seldin, H. Yin, E. C. Baechler, Q.-Z. Li, E. K. Wakeland, G. R. Bruner, K. M. Kaufman, and J. A. Kelly. 2008. Genome-wide association scan in women with systemic lupus erythematosus identifies susceptibility variants in ITGAM, PXK, KIAA1542 and other loci. Nature genetics 40: 204-210.

120. Kozyrev, S. V., A.-K. Abelson, J. Wojcik, A. Zaghlool, M. V. P. Linga Reddy, E. Sanchez, I. Gunnarsson, E. Svenungsson, G. Sturfelt, A. Jönsen, L. Truedsson, B. A. Pons-Estel, T. Witte, S. D'Alfonso, N. Barizzone, M. G. Danieli, C. Gutierrez, A. Suarez, P. Junker, H. Laustrup, M. F. González-Escribano, J. Martin, H. Abderrahim, and M. E. Alarcón-Riquelme. 2008. Functional variants in the B-cell gene BANK1 are associated with systemic lupus erythematosus. Nature Genetics 40: 211.

121. Nündel, K., N. M. Green, A. L. Shaffer, K. L. Moody, P. Busto, D. Eilat, K. Miyake, M. A. Oropallo, M. P. Cancro, and A. Marshak-Rothstein. 2015. CellIntrinsic Expression of TLR9 in Autoreactive B Cells Constrains BCR/TLR7Dependent Responses. The Journal of Immunology 194: 2504-2512.

122. Hofmann, K., A.-K. Clauder, and R. A. Manz. 2018. Targeting B Cells and Plasma Cells in Autoimmune Diseases. Frontiers in Immunology 9: 835.

123. Walsh, E. R., P. Pisitkun, E. Voynova, J. A. Deane, B. L. Scott, R. R. Caspi, and S. Bolland. 2012. Dual signaling by innate and adaptive immune receptors is required for TLR7-induced B-cell-mediated autoimmunity. Proceedings of the National Academy of Sciences of the United States of America 109: 16276-16281. 
124. Nath, S. K., S. Han, X. Kim-Howard, J. A. Kelly, P. Viswanathan, G. S. Gilkeson, W. Chen, C. Zhu, R. P. McEver, R. P. Kimberly, M. E. Alarcón-Riquelme, T. J. Vyse, Q.-Z. Li, E. K. Wakeland, J. T. Merrill, J. A. James, K. M. Kaufman, J. M. Guthridge, and J. B. Harley. 2008. A nonsynonymous functional variant in integrin- $\alpha \mathrm{M}$ (encoded by ITGAM) is associated with systemic lupus erythematosus. Nature Genetics 40: 152.

125. Ross, G. D. 2002. Role of the lectin domain of Mac-1/CR3 (CD11b/CD18) in regulating intercellular adhesion. Immunologic Research 25: 219-227.

126. Sugimori, T., D. L. Griffith, and M. A. Arnaout. 1997. Emerging paradigms of integrin ligand binding and activation. Kidney Int 51: 1454-1462.

127. Merle, N. S., R. Noe, L. Halbwachs-Mecarelli, V. Fremeaux-Bacchi, and L. T. Roumenina. 2015. Complement System Part II: Role in Immunity. Frontiers in Immunology 6: 257.

128. Gahmberg, C. G., S. C. Fagerholm, S. M. Nurmi, T. Chavakis, S. Marchesan, and M. Grönholm. 2009. Regulation of integrin activity and signalling. Biochimica et Biophysica Acta (BBA) - General Subjects 1790: 431-444.

129. Kim, C., F. Ye, and M. H. Ginsberg. 2011. Regulation of Integrin Activation. Annual Review of Cell and Developmental Biology 27: 321-345.

130. Podgrabinska, S., O. Kamalu, L. Mayer, M. Shimaoka, H. Snoeck, G. J. Randolph, and M. Skobe. 2009. Inflamed Lymphatic Endothelium Suppresses Dendritic Cell Maturation and Function via Mac-1/ICAM-1-Dependent Mechanism. The Journal of Immunology 183: 1767-1779.

131. Han, C., J. Jin, S. Xu, H. Liu, N. Li, and X. Cao. 2010. Integrin CD11b negatively regulates TLR-triggered inflammatory responses by activating Syk and promoting degradation of MyD88 and TRIF via Cbl-b. Nature Immunology 11: 734.

132. Wang, L., R. A. Gordon, L. Huynh, X. Su, K.-H. P. Min, J. Han, J. S. Arthur, G. D. Kalliolias, and L. B. Ivashkiv. 2010. Indirect Inhibition of Toll-like Receptor and Type I Interferon Responses by ITAM-Coupled Receptors and Integrins. Immunity 32: 518-530.

133. Zhang, M., Y. Han, C. Han, S. Xu, Y. Bao, Z. Chen, Y. Gu, D. Xia, and X. Cao. 2009. The $\beta 2$ integrin CD11b attenuates polyinosinic:Polycytidylic acid-induced hepatitis by negatively regulating natural killer cell functions. Hepatology 50: 1606-1616.

134. Ehirchiou, D., Y. Xiong, G. Xu, W. Chen, Y. Shi, and L. Zhang. 2007. CD11b facilitates the development of peripheral tolerance by suppressing Th17 differentiation. The Journal of Experimental Medicine 204: 1519-1524.

135. Kevil, C. G., M. J. Hicks, X. He, J. Zhang, C. M. Ballantyne, C. Raman, T. R. Schoeb, and D. C. Bullard. 2004. Loss of LFA-1, but not Mac-1, Protects MRL/MpJ-<em $>$ Fas $<$ sup $>$ lpr $</$ sup $></$ em $>$ Mice from Autoimmune Disease. The American Journal of Pathology 165: 609-616.

136. Ding, C., Y. Ma, X. Chen, M. Liu, Y. Cai, X. Hu, D. Xiang, S. Nath, H.-g. Zhang, H. Ye, D. Powell, and J. Yan. 2013. Integrin CD11b negatively regulates BCR signalling to maintain autoreactive B cell tolerance. Nat Commun 4: 2813.

137. Rush, J. S., and P. D. Hodgkin. 2001. B cells activated via CD40 and IL-4 undergo a division burst but require continued stimulation to maintain division, survival and differentiation. European Journal of Immunology 31: 1150-1159. 
138. Turner, M. L., E. D. Hawkins, and P. D. Hodgkin. 2008. Quantitative Regulation of B Cell Division Destiny by Signal Strength. The Journal of Immunology 181: 374-382.

139. Surova, E., and H. Jumaa. 2014. Chapter Four - The Role of BCR Isotype in BCell Development and Activation. In Advances in Immunology. H. L. Ploegh, ed. Academic Press. 101-139.

140. Chan, T. D., and R. Brink. 2012. Affinity-based selection and the germinal center response. Immunological Reviews 247: 11-23.

141. Batista, F. D., and M. S. Neuberger. 2000. B cells extract and present immobilized antigen: implications for affinity discrimination. The EMBO Journal 19: 513-520.

142. Khalil, A. M., J. C. Cambier, and M. J. Shlomchik. 2012. B Cell Receptor Signal Transduction in the GC Is Short-Circuited by High Phosphatase Activity. Science 336: 1178-1181.

143. Mueller, J., M. Matloubian, and J. Zikherman. 2015. Cutting edge: An in vivo reporter reveals active B cell receptor signaling in the germinal center. Journal of immunology (Baltimore, Md. : 1950) 194: 2993-2997.

144. Luo, W., F. Weisel, and M. J. Shlomchik. 2018. B Cell Receptor and CD40 Signaling Are Rewired for Synergistic Induction of the c-Myc Transcription Factor in Germinal Center B Cells. Immunity 48: 313-326.e315.

145. Dominguez-Sola, D., J. Kung, Antony B. Holmes, Victoria A. Wells, T. Mo, K. Basso, and R. Dalla-Favera. 2015. The FOXO1 Transcription Factor Instructs the Germinal Center Dark Zone Program. Immunity 43: 1064-1074.

146. Calado, D. P., Y. Sasaki, S. A. Godinho, A. Pellerin, K. Köchert, B. P. Sleckman, I. M. de Alborán, M. Janz, S. Rodig, and K. Rajewsky. 2012. The cell-cycle regulator c-Myc is essential for the formation and maintenance of germinal centers. Nature Immunology 13: 1092.

147. Takeshi, T. 2012. Role of Inhibitory BCR Co-Receptors in Immunity. Infectious Disorders - Drug Targets 12: 181-190.

148. Condé, C., G. Gloire, and J. Piette. 2011. Enzymatic and non-enzymatic activities of SHIP-1 in signal transduction and cancer. Biochemical Pharmacology 82: 1320-1334.

149. Dustin, L. B., D. R. Plas, J. Wong, Y. T. Hu, C. Soto, A. C. Chan, and M. L. Thomas. 1999. Expression of Dominant-Negative Src-Homology Domain 2Containing Protein Tyrosine Phosphatase-1 Results in Increased Syk Tyrosine Kinase Activity and B Cell Activation. The Journal of Immunology 162: 27172724.

150. Tsubata, T. 2018. Ligand Recognition Determines the Role of Inhibitory B Cell Co-receptors in the Regulation of B Cell Homeostasis and Autoimmunity.

Frontiers in Immunology 9: 2276.

151. Myers, D. R., J. Zikherman, and J. P. Roose. 2017. Tonic Signals: Why Do Lymphocytes Bother? Trends in Immunology 38: 844-857.

152. Hutzler, S., L. Özgör, Y. Naito-Matsui, K. Kläsener, T. H. Winkler, M. Reth, and L. Nitschke. 2014. The Ligand-Binding Domain of Siglec-G Is Crucial for Its Selective Inhibitory Function on B1 Cells. The Journal of Immunology 192: 54065414. 
153. Coffman, R. L., A. Sher, and R. A. Seder. 2010. Vaccine Adjuvants: Putting Innate Immunity to Work. Immunity 33: 492-503.

154. Bassi, N., R. Luisetto, A. Ghirardello, M. Gatto, B. Bottazzi, Y. Shoenfeld, L. Punzi, and A. Doria. 2012. Vaccination of mice for research purpose: Alum is as effective as and safer than complete Freund adjuvant.

155. Abram, C. L., G. L. Roberge, Y. Hu, and C. A. Lowell. 2014. Comparative analysis of the efficiency and specificity of myeloid-Cre deleting strains using ROSA-EYFP reporter mice. Journal of immunological methods 408: 89-100.

156. Wolniak, K. L., R. J. Noelle, and T. J. Waldschmidt. 2006. Characterization of (4Hydroxy-3-Nitrophenyl)Acetyl (NP)-Specific Germinal Center B Cells and Antigen-Binding B220<sup $>-</$ sup $>$ Cells after Primary NP Challenge in Mice. The Journal of Immunology 177: 2072-2079.

157. Paus, D., T. G. Phan, T. D. Chan, S. Gardam, A. Basten, and R. Brink. 2006. Antigen recognition strength regulates the choice between extrafollicular plasma cell and germinal center B cell differentiation. The Journal of Experimental Medicine 203: 1081-1091.

158. Mesin, L., J. Ersching, and G. D. Victora. 2016. Germinal Center B Cell Dynamics. Immunity 45: 471-482.

159. Smith, K. G. C., A. Light, L. A. O'Reilly, S.-M. Ang, A. Strasser, and D. Tarlinton. 2000. bcl-2 Transgene Expression Inhibits Apoptosis in the Germinal Center and Reveals Differences in the Selection of Memory B Cells and Bone Marrow Antibody-Forming Cells. The Journal of Experimental Medicine 191: 475-484.

160. Takahashi, Y., D. M. Cerasoli, J. M. Dal Porto, M. Shimoda, R. Freund, W. Fang, D. G. Telander, E.-N. Malvey, D. L. Mueller, T. W. Behrens, and G. Kelsoe. 1999. Relaxed Negative Selection in Germinal Centers and Impaired Affinity Maturation in bcl-x (L) Transgenic Mice. The Journal of Experimental Medicine 190: 399-410.

161. Gaudette, B. T., N. N. Iwakoshi, and L. H. Boise. 2014. Bcl-xL Protein Protects from C/EBP Homologous Protein (CHOP)-dependent Apoptosis during Plasma Cell Differentiation. Journal of Biological Chemistry 289: 23629-23640.

162. Cattoretti, G., C. Angelin-Duclos, R. Shaknovich, H. Zhou, D. Wang, and B. Alobeid. 2005. PRDM1/Blimp-1 is expressed in human B-lymphocytes committed to the plasma cell lineage. The Journal of Pathology 206: 76-86.

163. Martins, G., and K. Calame. 2008. Regulation and Functions of Blimp-1 in T and B Lymphocytes. Annual Review of Immunology 26: 133-169.

164. Sen, R. 2006. Control of B Lymphocyte Apoptosis by the Transcription Factor NF-\&\#x3ba;B. Immunity 25: 871-883.

165. Ettinger, R., G. P. Sims, A.-M. Fairhurst, R. Robbins, Y. S. da Silva, R. Spolski, W. J. Leonard, and P. E. Lipsky. 2005. IL-21 Induces Differentiation of Human Naive and Memory B Cells into Antibody-Secreting Plasma Cells. The Journal of Immunology 175: 7867-7879.

166. Depoil, D., and M. L. Dustin. 2016. Agile CD22 nanoclusters run rings around fenced BCR. The EMBO journal 35: 237-238. 
167. Buchner, M., and M. Müschen. 2014. Targeting the B cell receptor signaling pathway in B lymphoid malignancies. Current opinion in hematology 21: 341349.

168. Fiala, G. J., D. Kaschek, B. Blumenthal, M. Reth, J. Timmer, and W. W. A. Schamel. 2013. Pre-Clustering of the B Cell Antigen Receptor Demonstrated by Mathematically Extended Electron Microscopy. Frontiers in Immunology 4: 427.

169. Kawai, K., N. H. Tsuno, M. Matsuhashi, J. Kitayama, T. Osada, J. Yamada, T. Tsuchiya, S. Yoneyama, T. Watanabe, K. Takahashi, and H. Nagawa. 2005. CD11b-mediated migratory property of peripheral blood B cells. Journal of Allergy and Clinical Immunology 116: 192-197.

170. Zhang, X., Q. Wang, Y. Bi, Z. Kou, J. Zhou, Y. Cui, Y. Yan, L. Zhou, Y. Tan, H. Yang, Z. Du, Y. Han, Y. Song, P. Zhang, D. Zhou, R. Yang, and X. Wang. 2014. Kinetics of Memory B Cell and Plasma Cell Responses in the Mice Immunized with Plague Vaccines. Scandinavian Journal of Immunology 79: 157-162.

171. Nduati, E. W., D. H. L. Ng, F. M. Ndungu, P. Gardner, B. C. Urban, and J. Langhorne. 2010. Distinct Kinetics of Memory B-Cell and Plasma-Cell Responses in Peripheral Blood Following a Blood-Stage Plasmodium chabaudi Infection in Mice. PLOS ONE 5: e15007.

172. Pillai, S., and A. Cariappa. 2009. The follicular versus marginal zone B lymphocyte cell fate decision. Nature Reviews Immunology 9: 767.

173. Samardzic, T., D. Marinkovic, C.-P. Danzer, J. Gerlach, L. Nitschke, and T. Wirth. 2002. Reduction of marginal zone B cells in CD22-deficient mice. European Journal of Immunology 32: 561-567.

174. Rimaniol, A.-C., G. Gras, F. Verdier, F. Capel, V. B. Grigoriev, F. Porcheray, E. Sauzeat, J.-G. Fournier, P. Clayette, C.-A. Siegrist, and D. Dormont. 2004. Aluminum hydroxide adjuvant induces macrophage differentiation towards a specialized antigen-presenting cell type. Vaccine 22: 3127-3135.

175. Jordan, M. B., D. M. Mills, J. Kappler, P. Marrack, and J. C. Cambier. 2004. Promotion of B Cell Immune Responses via an Alum-Induced Myeloid Cell Population. Science 304: 1808-1810.

176. Xu, X., Q. Meng, U. Erben, P. Wang, R. Glauben, A. A. Kühl, H. Wu, C. W. Ma, M. Hu, Y. Wang, W. Sun, J. Jia, X. Wu, W. Chen, B. Siegmund, and Z. Qin. 2017. Myeloid-derived suppressor cells promote B-cell production of IgA in a TNFR2-dependent manner. Cellular and Molecular Immunology 14: 597-606.

177. Lelis, F. J. N., J. Jaufmann, A. Singh, K. Fromm, A. C. Teschner, S. Pöschel, I. Schäfer, S. Beer-Hammer, N. Rieber, and D. Hartl. 2017. Myeloid-derived suppressor cells modulate B-cell responses. Immunology Letters 188: 108-115.

178. Trigunaite, A., A. Khan, E. Der, A. Song, S. Varikuti, and T. N. JØrgensen. 2013. Gr-1highCD11b+ Cells Suppress B Cell Differentiation and Lupus-like Disease in Lupus-Prone Male Mice. Arthritis \& Rheumatism 65: 2392-2402.

179. Bird, A. K., M. Chang, J. Barnard, B. I. Goldman, N. Meednu, J. Rangel-Moreno, and J. H. Anolik. 2017. Neutrophils Slow Disease Progression in Murine Lupus via Modulation of Autoreactive Germinal Centers. The Journal of Immunology 199: 458-466. 
180. Jablonska, J., K. E. Dittmar, T. Kleinke, J. Buer, and S. Weiss. 2007. Essential role of CCL2 in clustering of splenic ERTR-9+ macrophages during infection of BALB/c mice by Listeria monocytogenes. Infection and immunity 75: 462-470.

181. Nikbakht, N., S. Shen, and T. Manser. 2013. Cutting edge: Macrophages are required for localization of antigen-activated $\mathrm{B}$ cells to the follicular perimeter and the subsequent germinal center response. Journal of immunology (Baltimore, Md. : 1950) 190: 4923-4927.

182. Melchers, F. 2005. The pre-B-cell receptor: selector of fitting immunoglobulin heavy chains for the B-cell repertoire. Nature Reviews Immunology 5: 578.

183. Hess, J., A. Werner, T. Wirth, F. Melchers, H.-M. Jäck, and T. H. Winkler. 2001. Induction of pre-B cell proliferation after $\langle\mathrm{em}>$ de novo $</ \mathrm{em}>$ synthesis of the pre-B cell receptor. Proceedings of the National Academy of Sciences 98: 17451750.

184. Nemazee, D., and M. Weigert. 2000. Revising B cell receptors. J Exp Med 191: 1813-1817.

185. Niiro, H., and E. A. Clark. 2002. Regulation of B-cell fate by antigen-receptor signals. Nature Reviews Immunology 2: 945.

186. Sáez de Guinoa, J., L. Barrio, M. Mellado, and Y. R. Carrasco. 2011. CXCL13/CXCR5 signaling enhances BCR-triggered B-cell activation by shaping cell dynamics. Blood 118: 1560-1569.

187. Calpe, E., C. Codony, M. J. Baptista, P. Abrisqueta, C. Carpio, N. Purroy, F. Bosch, and M. Crespo. 2011. ZAP-70 enhances migration of malignant B lymphocytes toward CCL21 by inducing CCR7 expression via IgM-ERK1/2 activation. Blood 118: 4401-4410.

188. Postigo, A. A., A. L. Corbí, F. Sánchez-Madrid, and M. O. de Landázuri. 1991.

Regulated expression and function of CD11c/CD18 integrin on human B lymphocytes. Relation between attachment to fibrinogen and triggering of proliferation through CD11c/CD18. The Journal of Experimental Medicine 174: 1313-1322.

189. Griffin, D. O., and T. L. Rothstein. 2011. A small CD11b(+) human B1 cell subpopulation stimulates T cells and is expanded in lupus. The Journal of Experimental Medicine 208: 2591-2598.

190. Griffin, D. O., and T. L. Rothstein. 2012. Human “Orchestrator” CD11b(+) B1 Cells Spontaneously Secrete Interleukin-10 and Regulate T-Cell Activity. Molecular Medicine 18: 1003-1008.

191. Ghosn, E. E. B., Y. Yang, J. Tung, L. A. Herzenberg, and L. A. Herzenberg. 2008. CD11b expression distinguishes sequential stages of peritoneal B-1 development. Proceedings of the National Academy of Sciences 105: 5195-5200.

192. Liu, X., X. Jiang, R. Liu, L. Wang, T. Qian, Y. Zheng, Y. Deng, E. Huang, F. Xu, J.-Y. Wang, and Y. Chu. 2015. B cells expressing CD11b effectively inhibit CD4+ T-cell responses and ameliorate experimental autoimmune hepatitis in mice. Hepatology 62: 1563-1575.

193. Kunisawa, J., M. Gohda, E. Hashimoto, I. Ishikawa, M. Higuchi, Y. Suzuki, Y. Goto, C. Panea, I. I. Ivanov, R. Sumiya, L. Aayam, T. Wake, S. Tajiri, Y. Kurashima, S. Shikata, S. Akira, K. Takeda, and H. Kiyono. 2013. Microbe- 
dependent $\mathrm{CD} 11 \mathrm{~b}(+) \operatorname{IgA}(+)$ plasma cells mediate robust early-phase intestinal IgA responses in mice. Nat Commun 4: 1772.

194. Jacobson, A. C., K. M. Roundy, J. J. Weis, and J. H. Weis. 2009. Regulation of Murine Splenic B Cell CR3 Expression by Complement Component 3. The Journal of Immunology 183: 3963-3970.

195. Watanabe-Fukunaga, R., C. I. Brannan, N. G. Copeland, N. A. Jenkins, and S. Nagata. 1992. Lymphoproliferation disorder in mice explained by defects in Fas antigen that mediates apoptosis. Nature 356: 314.

196. Richard, M. L., and G. Gilkeson. 2018. Mouse models of lupus: what they tell us and what they don't. Lupus Science \& Medicine 5: e000199.

197. Reddy, S. T., X. Ge, A. E. Miklos, R. A. Hughes, S. H. Kang, K. H. Hoi, C. Chrysostomou, S. P. Hunicke-Smith, B. L. Iverson, P. W. Tucker, A. D. Ellington, and G. Georgiou. 2010. Monoclonal antibodies isolated without screening by analyzing the variable-gene repertoire of plasma cells. Nature Biotechnology 28: 965.

198. Zuckerman, N. S., H. Hazanov, M. Barak, H. Edelman, S. Hess, H. Shcolnik, D. Dunn-Walters, and R. Mehr. 2010. Somatic hypermutation and antigen-driven selection of B cells are altered in autoimmune diseases. Journal of Autoimmunity 35: 325-335.

199. Kawasaki, A., S. Ito, H. Furukawa, T. Hayashi, D. Goto, I. Matsumoto, M. Kusaoi, J. Ohashi, R. R. Graham, K. Matsuta, T. W. Behrens, S. Tohma, Y. Takasaki, H. Hashimoto, T. Sumida, and N. Tsuchiya. 2010. Association of TNFAIP3 interacting protein 1, TNIP1 with systemic lupus erythematosus in a Japanese population: a case-control association study. Arthritis Research \& Therapy 12: R174-R174.

200. Caster, D. J., E. A. Korte, S. K. Nanda, K. R. McLeish, R. K. Oliver, R. T. G'Sell, R. M. Sheehan, D. W. Freeman, S. C. Coventry, J. A. Kelly, J. M. Guthridge, J. A. James, K. L. Sivils, M. E. Alarcon-Riquelme, R. H. Scofield, I. Adrianto, P. M. Gaffney, A. M. Stevens, B. I. Freedman, C. D. Langefeld, B. P. Tsao, B. A. Pons-Estel, C. O. Jacob, D. L. Kamen, G. S. Gilkeson, E. E. Brown, G. S. Alarcon, J. C. Edberg, R. P. Kimberly, J. Martin, J. T. Merrill, J. B. Harley, K. M. Kaufman, J. D. Reveille, J.-M. Anaya, L. A. Criswell, L. M. Vila, M. Petri, R. Ramsey-Goldman, S.-C. Bae, S. A. Boackle, T. J. Vyse, T. B. Niewold, P. Cohen, and D. W. Powell. 2013. ABIN1 Dysfunction as a Genetic Basis for Lupus Nephritis. Journal of the American Society of Nephrology: JASN 24: 1743-1754.

201. Zhou, J., R. Wu, A. A. High, C. A. Slaughter, D. Finkelstein, J. E. Rehg, V. Redecke, and H. Häcker. 2011. A20-binding inhibitor of NF- $\kappa B$ (ABIN1) controls Toll-like receptor-mediated CCAAT/enhancer-binding protein $\beta$ activation and protects from inflammatory disease. Proceedings of the National Academy of Sciences 108: E998-E1006.

202. Korte, E. A., D. J. Caster, M. T. Barati, M. Tan, S. Zheng, C. C. Berthier, F. C. Brosius, III, M. B. Vieyra, R. M. Sheehan, M. Kosiewicz, M. Wysoczynski, P. M. Gaffney, D. J. Salant, K. R. McLeish, and D. W. Powell. 2017. ABIN1 Determines Severity of Glomerulonephritis via Activation of Intrinsic Glomerular Inflammation. The American Journal of Pathology 187: 2799-2810. 
203. Nanda, S. K., R. K. C. Venigalla, A. Ordureau, J. C. Patterson-Kane, D. W. Powell, R. Toth, J. S. C. Arthur, and P. Cohen. 2011. Polyubiquitin binding to ABIN1 is required to prevent autoimmunity. The Journal of Experimental Medicine 208: 1215-1228.

204. Mandik-Nayak, L., S.-j. Seo, C. Sokol, K. M. Potts, A. Bui, and J. Erikson. 1999. MRL-<em >lpr/lpr</em> Mice Exhibit a Defect in Maintaining Developmental Arrest and Follicular Exclusion of Anti-double-stranded DNA B Cells. The Journal of Experimental Medicine 189: 1799-1814.

205. Manz, R. A., M. Lohning, G. Cassese, A. Thiel, and A. Radbruch. 1998. Survival of long-lived plasma cells is independent of antigen. Int Immunol 10: 1703-1711.

206. Morawski, P. A., and S. Bolland. 2017. Expanding the B Cell Centric View of Systemic Lupus Erythematosus. Trends in immunology 38: 373-382.

207. Rhodes, B., B. G. Fürnrohr, A. L. Roberts, G. Tzircotis, G. Schett, T. D. Spector, and T. J. Vyse. 2012. The rs1143679 (R77H) lupus associated variant of ITGAM (CD11b) impairs complement receptor 3 mediated functions in human monocytes. Annals of the Rheumatic Diseases 71: 2028-2034.

208. Faridi, M. H., S. Q. Khan, W. Zhao, H. W. Lee, M. M. Altintas, K. Zhang, V. Kumar, A. R. Armstrong, C. Carmona-Rivera, J. M. Dorschner, A. M. Schnaith, X. Li, Y. Ghodke-Puranik, E. Moore, M. Purmalek, J. Irizarry-Caro, T. Zhang, R. Day, D. Stoub, V. Hoffmann, S. J. Khaliqdina, P. Bhargava, A. M. Santander, M. Torroella-Kouri, B. Issac, D. J. Cimbaluk, A. Zloza, R. Prabhakar, S. Deep, M. Jolly, K. H. Koh, J. S. Reichner, E. M. Bradshaw, J. Chen, L. F. Moita, P. S. Yuen, W. Li Tsai, B. Singh, J. Reiser, S. K. Nath, T. B. Niewold, R. I. VazquezPadron, M. J. Kaplan, and V. Gupta. 2017. CD11b activation suppresses TLRdependent inflammation and autoimmunity in systemic lupus erythematosus. The Journal of Clinical Investigation 127.

209. Gilliam, J. N., and R. D. Sontheimer. 1981. Distinctive cutaneous subsets in the spectrum of lupus erythematosus. J Am Acad Dermatol 4: 471-475.

210. Kuhn, A., G. Bonsmann, H.-J. Anders, P. Herzer, K. Tenbrock, and M. Schneider. 2015. The Diagnosis and Treatment of Systemic Lupus Erythematosus. Deutsches Ärzteblatt International 112: 423-432.

211. Cervera, R., M. A. Khamashta, J. Font, G. D. Sebastiani, A. Gil, P. Lavilla, I. Domenech, A. O. Aydintug, A. Jedryka-Goral, E. de Ramon, and et al. 1993. Systemic lupus erythematosus: clinical and immunologic patterns of disease expression in a cohort of 1,000 patients. The European Working Party on Systemic Lupus Erythematosus. Medicine (Baltimore) 72: 113-124.

212. Fischer-Betz, R., P. Herzer, and M. Schneider. 2005. Systemischer Lupus erythematodes. Dtsch med Wochenschr 130: 2451-2458.

213. Bertsias, G. K., J. P. A. Ioannidis, M. Aringer, E. Bollen, S. Bombardieri, I. N. Bruce, R. Cervera, M. Dalakas, A. Doria, J. G. Hanly, T. W. J. Huizinga, D. Isenberg, C. Kallenberg, J. C. Piette, M. Schneider, N. Scolding, J. Smolen, A. Stara, I. Tassiulas, M. Tektonidou, A. Tincani, M. A. van Buchem, R. van Vollenhoven, M. Ward, C. Gordon, and D. T. Boumpas. 2010. EULAR recommendations for the management of systemic lupus erythematosus with neuropsychiatric manifestations: report of a task force of the EULAR standing committee for clinical affairs. Annals of the Rheumatic Diseases 69: 2074-2082. 
214. Florica, B., E. Aghdassi, J. Su, D. D. Gladman, M. B. Urowitz, and P. R. Fortin. 2011. Peripheral Neuropathy in Patients with Systemic Lupus Erythematosus. Seminars in Arthritis and Rheumatism 41: 203-211.

215. Weening, J. J., V. D. D'Agati, M. M. Schwartz, S. V. Seshan, C. E. Alpers, G. B. Appel, J. E. Balow, J. A. Bruijn, T. Cook, F. Ferrario, A. B. Fogo, E. M. Ginzler, L. Hebert, G. Hill, P. Hill, J. C. Jennette, N. C. Kong, P. Lesavre, M. Lockshin, L. M. Looi, H. Makino, L. A. Moura, and M. Nagata. 2004. The classification of glomerulonephritis in systemic lupus erythematosus revisited. J Am Soc Nephrol 15: 241-250.

216. Bertsias, G. K., M. Tektonidou, Z. Amoura, M. Aringer, I. Bajema, J. H. M. Berden, J. Boletis, R. Cervera, T. Dörner, A. Doria, F. Ferrario, J. Floege, F. A. Houssiau, J. P. A. Ioannidis, D. A. Isenberg, C. G. M. Kallenberg, L. Lightstone, S. D. Marks, A. Martini, G. Moroni, I. Neumann, M. Praga, M. Schneider, A. Starra, V. Tesar, C. Vasconcelos, R. F. van Vollenhoven, H. Zakharova, M. Haubitz, C. Gordon, D. Jayne, and D. T. Boumpas. 2012. Joint European League Against Rheumatism and European Renal Association-European Dialysis and Transplant Association (EULAR/ERA-EDTA) recommendations for the management of adult and paediatric lupus nephritis. Annals of the Rheumatic Diseases 71: 1771-1782.

217. Morris, David L., Kimberly E. Taylor, Michelle M. A. Fernando, J. Nititham, Marta E. Alarcón-Riquelme, Lisa F. Barcellos, Timothy W. Behrens, C. Cotsapas, Patrick M. Gaffney, Robert R. Graham, Bernardo A. Pons-Estel, Peter K. Gregersen, John B. Harley, Stephen L. Hauser, G. Hom, Carl D. Langefeld, Janelle A. Noble, John D. Rioux, Michael F. Seldin, Lindsey A. Criswell, and Timothy J. Vyse. 2012. Unraveling Multiple MHC Gene Associations with Systemic Lupus Erythematosus: Model Choice Indicates a Role for HLA Alleles and Non-HLA Genes in Europeans. The American Journal of Human Genetics 91: 778-793.

218. Farh, K. K.-H., A. Marson, J. Zhu, M. Kleinewietfeld, W. J. Housley, S. Beik, N. Shoresh, H. Whitton, R. J. H. Ryan, A. A. Shishkin, M. Hatan, M. J. CarrascoAlfonso, D. Mayer, C. J. Luckey, N. A. Patsopoulos, P. L. De Jager, V. K. Kuchroo, C. B. Epstein, M. J. Daly, D. A. Hafler, and B. E. Bernstein. 2014. Genetic and epigenetic fine mapping of causal autoimmune disease variants. Nature 518: 337.

219. Harley, I. T. W., K. M. Kaufman, C. D. Langefeld, J. B. Harley, and J. A. Kelly. 2009. Genetic susceptibility to SLE: new insights from fine mapping and genomewide association studies. Nature Reviews Genetics 10: 285.

220. Wahl, R., T. Meo, D. Shreffler, W. Miller, J. P. Atkinson, J. Schultz, and C. K. Osterland. 1979. C2 deficiency and a lupus erythematosus-like illness: family reevaluation. Ann Intern Med 90: 717-718.

221. Pussell, B. A., E. Bourke, M. Nayef, S. Morris, and D. K. Peters. 1980. Complement deficiency and nephritis. A report of a family. Lancet 1: 675-677.

222. Yasutomo, K., T. Horiuchi, S. Kagami, H. Tsukamoto, C. Hashimura, M. Urushihara, and Y. Kuroda. 2001. Mutation of DNASE1 in people with systemic lupus erythematosus. Nature Genetics 28: 313. 
223. Xiang, N., X.-m. Li, G.-s. Wang, J.-h. Tao, and X.-p. Li. 2013. Association of Fas gene polymorphisms with systemic lupus erythematosus: a meta-analysis.

Molecular Biology Reports 40: 407-415.

224. Fagerholm, S. C., M. MacPherson, M. J. James, C. Sevier-Guy, and C. S. Lau. 2013. The CD11b-integrin (ITGAM) and systemic lupus erythematosus. Lupus 22: 657-663.

225. Hu, X., H. Kim, E. Stahl, R. Plenge, M. Daly, and S. Raychaudhuri. 2011. Integrating Autoimmune Risk Loci with Gene-Expression Data Identifies Specific Pathogenic Immune Cell Subsets. The American Journal of Human Genetics 89: 496-506.

226. Wardemann, H., and M. C. Nussenzweig. 2007. B-cell self-tolerance in humans. Adv Immunol 95: 83-110.

227. Mohan, C., and C. Putterman. 2015. Genetics and pathogenesis of systemic lupus erythematosus and lupus nephritis. Nature Reviews Nephrology 11: 329.

228. Domeier, P. P., S. L. Schell, and Z. S. M. Rahman. 2017. Spontaneous germinal centers and autoimmunity. Autoimmunity 50: 4-18.

229. Odendahl, M., A. Jacobi, A. Hansen, E. Feist, F. Hiepe, G. R. Burmester, P. E. Lipsky, A. Radbruch, and T. Dörner. 2000. Disturbed Peripheral B Lymphocyte Homeostasis in Systemic Lupus Erythematosus. The Journal of Immunology 165: 5970-5979.

230. Woods, M., Y.-R. Zou, and A. Davidson. 2015. Defects in Germinal Center Selection in SLE. Frontiers in Immunology 6: 425.

231. Ekland, E. H., R. Forster, M. Lipp, and J. G. Cyster. 2004. Requirements for Follicular Exclusion and Competitive Elimination of Autoantigen-Binding B Cells. The Journal of Immunology 172: 4700-4708.

232. Cappione, A., J. H. Anolik, A. Pugh-Bernard, J. Barnard, P. Dutcher, G. Silverman, and I. Sanz. 2005. Germinal center exclusion of autoreactive B cells is defective in human systemic lupus erythematosus. Journal of Clinical Investigation 115: 3205-3216.

233. Guo, W., D. Smith, K. Aviszus, T. Detanico, R. A. Heiser, and L. J. Wysocki. 2010. Somatic hypermutation as a generator of antinuclear antibodies in a murine model of systemic autoimmunity. The Journal of Experimental Medicine 207: 2225-2237.

234. Mietzner, B., M. Tsuiji, J. Scheid, K. Velinzon, T. Tiller, K. Abraham, J. B. Gonzalez, V. Pascual, D. Stichweh, H. Wardemann, and M. C. Nussenzweig. 2008. Autoreactive IgG memory antibodies in patients with systemic lupus erythematosus arise from nonreactive and polyreactive precursors. Proceedings of the National Academy of Sciences 105: 9727-9732.

235. Diamond, B., and M. D. Scharff. 1984. Somatic mutation of the T15 heavy chain gives rise to an antibody with autoantibody specificity. Proceedings of the National Academy of Sciences of the United States of America 81: 5841-5844.

236. Zhang, J., V. Roschke, K. P. Baker, Z. Wang, G. S. Alarcón, B. J. Fessler, H. Bastian, R. P. Kimberly, and T. Zhou. 2001. Cutting Edge: A Role for B Lymphocyte Stimulator in Systemic Lupus Erythematosus. The Journal of Immunology 166: 6-10. 
237. Gómez-Martín, D., M. Díaz-Zamudio, J. Romo-Tena, M. J. Ibarra-Sánchez, and J. Alcocer-Varela. 2011. Follicular helper T cells poise immune responses to the development of autoimmune pathology. Autoimmunity Reviews 10: 325-330.

238. Kashiwakuma, D., A. Suto, Y. Hiramatsu, K. Ikeda, H. Takatori, K. Suzuki, S.-i. Kagami, K. Hirose, N. Watanabe, I. Iwamoto, and H. Nakajima. 2010. B and T Lymphocyte Attenuator Suppresses IL-21 Production from Follicular Th Cells and Subsequent Humoral Immune Responses. The Journal of Immunology 185: 2730-2736.

239. Cortini, A., U. Ellinghaus, T. H. Malik, D. S. Cunninghame Graham, M. Botto, and T. J. Vyse. 2017. B cell OX40L supports T follicular helper cell development and contributes to SLE pathogenesis. Annals of the Rheumatic Diseases 76: 20952103.

240. Bentham, J., D. L. Morris, D. S. C. Graham, C. L. Pinder, P. Tombleson, T. W. Behrens, J. Martín, B. P. Fairfax, J. C. Knight, L. Chen, J. Replogle, A.-C. Syvänen, L. Rönnblom, R. R. Graham, J. E. Wither, J. D. Rioux, M. E. AlarcónRiquelme, and T. J. Vyse. 2015. Genetic association analyses implicate aberrant regulation of innate and adaptive immunity genes in the pathogenesis of systemic lupus erythematosus. Nature genetics 47: 1457-1464.

241. Yang, W., M. Zhao, N. Hirankarn, C. S. Lau, C. C. Mok, T. M. Chan, R. W. S. Wong, K. W. Lee, M. Y. Mok, S. N. Wong, Y. Avihingsanon, I. O. L. N.G, T. L. Lee, M. H. K. Ho, P. P. W. Lee, W. H. S. Wong, P. C. Sham, and Y. L. Lau. 2009. ITGAM is associated with disease susceptibility and renal nephritis of systemic lupus erythematosus in Hong Kong Chinese and Thai. Human Molecular Genetics 18: 2063-2070.

242. Han, S., X. Kim-Howard, H. Deshmukh, Y. Kamatani, P. Viswanathan, J. M. Guthridge, K. Thomas, K. M. Kaufman, J. Ojwang, A. Rojas-Villarraga, V. Baca, L. Orozco, B. Rhodes, C.-B. Choi, P. K. Gregersen, J. T. Merrill, J. A. James, P. M. Gaffney, K. L. Moser, C. O. Jacob, R. P. Kimberly, J. B. Harley, S.-C. Bae, J.M. Anaya, M. E. Alarcón-Riquelme, K. Matsuda, T. J. Vyse, and S. K. Nath. 2009. Evaluation of imputation-based association in and around the integrin- $\alpha-M$ (ITGAM) gene and replication of robust association between a non-synonymous functional variant within ITGAM and systemic lupus erythematosus (SLE). Human Molecular Genetics 18: 1171-1180.

243. Gupta, V., S. Kumar, A. Pratap, R. Singh, R. Kumari, S. Kumar, A. Aggarwal, and R. Misra. Association of ITGAM, TNFSF4, TNFAIP3 and STAT4 gene polymorphisms with risk of systemic lupus erythematosus in a North Indian population. Lupus 0: 0961203318786432.

244. Martin, D. A., and K. B. Elkon. 2005. Autoantibodies make a U-turn: the toll hypothesis for autoantibody specificity. The Journal of Experimental Medicine 202: 1465-1469.

245. Hammerberg, C., S. K. Katiyar, M. C. Carroll, and K. D. Cooper. 1998. Activated Complement Component 3 (C3) Is Required for Ultraviolet Induction of Immunosuppression and Antigenic Tolerance. The Journal of Experimental Medicine 187: 1133-1138.

246. MacPherson, M., H. S. Lek, A. Prescott, and S. C. Fagerholm. 2011. A Systemic Lupus Erythematosus-associated R77H Substitution in the CD11b Chain of the 
Mac-1 Integrin Compromises Leukocyte Adhesion and Phagocytosis. Journal of Biological Chemistry 286: 17303-17310.

247. Zhou, Y., J. Wu, D. F. Kucik, N. B. White, D. T. Redden, A. J. Szalai, D. C. Bullard, and J. C. Edberg. 2013. Multiple Lupus Associated ITGAM Variants Alter Mac-1 Function on Neutrophils. Arthritis and rheumatism 65: 2907-2916.

248. Rosetti, F., Y. Chen, M. Sen, E. Thayer, V. Azcutia, Jan M. Herter, F. W. Luscinskas, X. Cullere, C. Zhu, and Tanya N. Mayadas. 2015. A LupusAssociated Mac-1 Variant Has Defects in Integrin Allostery and Interaction with Ligands under Force. Cell Reports 10: 1655-1664.

249. Maiti, A. K., X. Kim-Howard, P. Motghare, V. Pradhan, K. H. Chua, C. Sun, M. T. Arango-Guerrero, K. Ghosh, T. B. Niewold, J. B. Harley, J.-M. Anaya, L. L. Looger, and S. K. Nath. 2014. Combined protein- and nucleic acid-level effects of rs $1143679(\mathrm{R} 77 \mathrm{H})$, a lupus-predisposing variant within ITGAM. Human Molecular Genetics 23: 4161-4176.

250. Roberts, A. L., B. G. Fürnrohr, T. J. Vyse, and B. Rhodes. 2016. The complement receptor 3 (CD11b/CD18) agonist Leukadherin-1 suppresses human innate inflammatory signalling. Clinical \& Experimental Immunology 185: 361-371.

251. Faridi, M. H., S. Q. Khan, W. Zhao, H. W. Lee, M. M. Altintas, K. Zhang, V. Kumar, A. R. Armstrong, C. Carmona-Rivera, J. M. Dorschner, A. M. Schnaith, X. Li, Y. Ghodke-Puranik, E. Moore, M. Purmalek, J. Irizarry-Caro, T. Zhang, R. Day, D. Stoub, V. Hoffmann, S. J. Khaliqdina, P. Bhargava, A. M. Santander, M. Torroella-Kouri, B. Issac, D. J. Cimbaluk, A. Zloza, R. Prabhakar, S. Deep, M. Jolly, K. H. Koh, J. S. Reichner, E. M. Bradshaw, J. Chen, L. F. Moita, P. S. Yuen, W. Li Tsai, B. Singh, J. Reiser, S. K. Nath, T. B. Niewold, R. I. VazquezPadron, M. J. Kaplan, and V. Gupta. 2017. CD11b activation suppresses TLRdependent inflammation and autoimmunity in systemic lupus erythematosus. The Journal of Clinical Investigation 127: 1271-1283.

252. MacPherson, M., H. S. Lek, A. Prescott, and S. C. Fagerholm. 2011. A Systemic Lupus Erythematosus-associated R77H Substitution in the CD11b Chain of the Mac-1 Integrin Compromises Leukocyte Adhesion and Phagocytosis. The Journal of biological chemistry 286: 17303-17310.

253. Chung, S. A., K. E. Taylor, R. R. Graham, J. Nititham, A. T. Lee, W. A. Ortmann, C. O. Jacob, M. E. Alarcón-Riquelme, B. P. Tsao, J. B. Harley, P. M. Gaffney, K. L. Moser, Slegen, M. Petri, F. Y. Demirci, M. I. Kamboh, S. Manzi, P. K.

Gregersen, C. D. Langefeld, T. W. Behrens, and L. A. Criswell. 2011. Differential Genetic Associations for Systemic Lupus Erythematosus Based on Anti-dsDNA Autoantibody Production. PLoS Genet 7: e1001323.

254. Toller-Kawahisa, J. E., I. C. C. Vigato-Ferreira, J. A. T. Pancoto, C. T. MendesJunior, E. Z. Martinez, G. M. Palomino, P. Louzada-Júnior, E. A. Donadi, J. E. C. Del Lama, and C. M. Marzocchi-Machado. 2014. The variant of CD11b, rs1143679 within ITGAM, is associated with systemic lupus erythematosus and clinical manifestations in Brazilian patients. Human Immunology 75: 119-123.

255. Maiguel, D., M. H. Faridi, C. Wei, Y. Kuwano, K. M. Balla, D. Hernandez, C. J. Barth, G. Lugo, M. Donnelly, A. Nayer, L. F. Moita, S. Schürer, D. Traver, P. Ruiz, R. I. Vazquez-Padron, K. Ley, J. Reiser, and V. Gupta. 2011. Small 
Molecule-Mediated Activation of the Integrin CD11b/CD18 Reduces

Inflammatory Disease. Science signaling 4: ra57-ra57.

256. Kim-Howard, X., A. K. Maiti, J.-M. Anaya, G. R. Bruner, E. Brown, J. T. Merrill, J. C. Edberg, M. A. Petri, J. D. Reveille, R. Ramsey-Goldman, G. S. Alarcon, T. J. Vyse, G. Gilkeson, R. P. Kimberly, J. A. James, J. M. Guthridge, J. B. Harley, and S. K. Nath. 2010. ITGAM coding variant (rs1 143679) influences the risk of renal disease, discoid rash, and immunologic manifestations in lupus patients with European ancestry. Annals of the rheumatic diseases 69: 1329-1332.

257. Lee, Y. H., and S.-C. Bae. 2015. Association between the functional ITGAM rs1143679 G/A polymorphism and systemic lupus erythematosus/lupus nephritis or rheumatoid arthritis: an update meta-analysis. Rheumatology International 35: 815-823.

258. Sanchez, E., A. Nadig, B. C. Richardson, B. I. Freedman, K. M. Kaufman, J. A. Kelly, T. B. Niewold, D. L. Kamen, G. S. Gilkeson, J. T. Ziegler, C. D. Langefeld, G. S. Alarcón, J. C. Edberg, R. Ramsey-Goldman, M. Petri, E. E. Brown, R. P. Kimberly, J. D. Reveille, L. M. Vilá, J. T. Merrill, J.-M. Anaya, J. A. James, B. A. Pons-Estel, J. Martin, S.-Y. Park, S.-Y. Bang, S.-C. Bae, K. L. Moser, T. J. Vyse, L. A. Criswell, P. M. Gaffney, B. P. Tsao, C. O. Jacob, J. B. Harley, M. E. Alarcón-Riquelme, B. on behalf of, Genles, and A. H. Sawalha. 2011. Phenotypic associations of genetic susceptibility loci in systemic lupus erythematosus. Annals of the rheumatic diseases 70: 1752-1757.

259. Li, C., F. Tong, Y. Ma, K. Qian, J. Zhang, and X. Chen. 2018. Association of the CD11b rs1143679 polymorphism with systemic lupus erythematosus in the Han Chinese population. The Journal of international medical research 46: 10081014.

260. Ceccarelli, F., C. Perricone, P. Borgiani, C. Ciccacci, S. Rufini, E. Cipriano, C. Alessandri, F. R. Spinelli, A. Sili Scavalli, G. Novelli, G. Valesini, and F. Conti. 2015. Genetic Factors in Systemic Lupus Erythematosus: Contribution to Disease Phenotype. Journal of immunology research 2015: 745647-745647.

261. Walport, M. J., and P. J. Lachmann. 1990. Complement deficiencies and abnormalities of the complement system in systemic lupus erythematosus and related disorders. Curr Opin Rheumatol 2: 661-663.

262. Liu, C.-C., A. H. Kao, S. Manzi, and J. M. Ahearn. 2013. Biomarkers in systemic lupus erythematosus: challenges and prospects for the future. Therapeutic advances in musculoskeletal disease 5: 210-233.

263. Schroeder, H. W., and L. Cavacini. 2010. Structure and Function of Immunoglobulins. The Journal of allergy and clinical immunology 125: S41-S52.

264. Chang, T. W., P. C. Wu, C. L. Hsu, and A. F. Hung. 2007. Anti-IgE Antibodies for the Treatment of IgE-Mediated Allergic Diseases. In Advances in Immunology. Academic Press. 63-119.

265. Kane, A., E. K. Deenick, C. S. Ma, M. C. Cook, G. Uzel, and S. G. Tangye. 2014. STAT3 is a central regulator of lymphocyte differentiation and function. Current Opinion in Immunology 28: 49-57.

266. Holland, S. M., F. R. DeLeo , H. Z. Elloumi , A. P. Hsu , G. Uzel , N. Brodsky , A. F. Freeman, A. Demidowich, J. Davis , M. L. Turner, V. L. Anderson, D. N. Darnell , P. A. Welch, D. B. Kuhns, D. M. Frucht, H. L. Malech, J. I. Gallin , S. 
D. Kobayashi , A. R. Whitney, J. M. Voyich , J. M. Musser , C. Woellner, A. A. Schäffer, J. M. Puck, and B. Grimbacher 2007. STAT3 Mutations in the HyperIgE Syndrome. New England Journal of Medicine 357: 1608-1619.

267. Minegishi, Y., M. Saito, S. Tsuchiya, I. Tsuge, H. Takada, T. Hara, N.

Kawamura, T. Ariga, S. Pasic, O. Stojkovic, A. Metin, and H. Karasuyama. 2007. Dominant-negative mutations in the DNA-binding domain of STAT3 cause hyper-IgE syndrome. Nature 448: 1058-1062.

268. Nojima, T., K. Haniuda, T. Moutai, M. Matsudaira, S. Mizokawa, I. Shiratori, T. Azuma, and D. Kitamura. 2011. In-vitro derived germinal centre B cells differentially generate memory B or plasma cells in vivo. Nat Commun 2: 465.

269. Yoon, S. O., X. Zhang, P. Berner, and S. Choi Yong. 2009. IL-21 and IL-10 have redundant roles but differential capacities at different stages of plasma cell generation from human germinal center B cells. Journal of Leukocyte Biology 86: 1311-1318.

270. Wu, H., L.-L. Xu, P. Teuscher, H. Liu, M. H. Kaplan, and A. L. Dent. 2015. An Inhibitory Role for the Transcription Factor Stat3 in Controlling IL-4 and Bcl6 Expression in Follicular Helper T Cells. The Journal of Immunology 195: 20802089.

271. Stritesky, G. L., R. Muthukrishnan, S. Sehra, R. Goswami, D. Pham, J. Travers, E. T. Nguyen, D. E. Levy, and M. H. Kaplan. 2011. The Transcription Factor STAT3 Is Required for T Helper 2 Cell Development. Immunity 34: 39-49.

272. Ma, C. S., D. T. Avery, A. Chan, M. Batten, J. Bustamante, S. Boisson-Dupuis, P. D. Arkwright, A. Y. Kreins, D. Averbuch, D. Engelhard, K. Magdorf, S. S. Kilic, Y. Minegishi, S. Nonoyama, M. A. French, S. Choo, J. M. Smart, J. Peake, M. Wong, P. Gray, M. C. Cook, D. A. Fulcher, J. L. Casanova, E. K. Deenick, and S. G. Tangye. 2012. Functional STAT3 deficiency compromises the generation of human T follicular helper cells. Blood 119: 3997-4008.

273. Avery, D. T., E. K. Deenick, C. S. Ma, S. Suryani, N. Simpson, G. Y. Chew, T. D. Chan, U. Palendira, J. Bustamante, S. Boisson-Dupuis, S. Choo, K. E. Bleasel, J. Peake, C. King, M. A. French, D. Engelhard, S. Al-Hajjar, S. Al-Muhsen, K. Magdorf, J. Roesler, P. D. Arkwright, P. Hissaria, D. S. Riminton, M. Wong, R. Brink, D. A. Fulcher, J.-L. Casanova, M. C. Cook, and S. G. Tangye. 2010. B cell-intrinsic signaling through IL-21 receptor and STAT3 is required for establishing long-lived antibody responses in humans. The Journal of Experimental Medicine 207: 155-171.

274. Deenick, E. K., D. T. Avery, A. Chan, L. J. Berglund, M. L. Ives, L. Moens, J. L. Stoddard, J. Bustamante, S. Boisson-Dupuis, M. Tsumura, M. Kobayashi, P. D. Arkwright, D. Averbuch, D. Engelhard, J. Roesler, J. Peake, M. Wong, S. Adelstein, S. Choo, J. M. Smart, M. A. French, D. A. Fulcher, M. C. Cook, C. Picard, A. Durandy, C. Klein, S. M. Holland, G. Uzel, J.-L. Casanova, C. S. Ma, and S. G. Tangye. 2013. Naive and memory human B cells have distinct requirements for STAT3 activation to differentiate into antibody-secreting plasma cells. The Journal of Experimental Medicine 210: 2739-2753.

275. Meyer-Bahlburg, A., E. D. Renner, S. Rylaarsdam, J. Reichenbach, L. F. Schimke, A. Marks, H. Tcheurekdjian, R. Hostoffer, A. Brahmandam, T. R. Torgerson, B. H. Belohradsky, D. J. Rawlings, and H. D. Ochs. 2012. 
Heterozygous signal transducer and activator of transcription 3 mutations in hyper-IgE syndrome result in altered B-cell maturation. Journal of Allergy and Clinical Immunology 129: 559-562.e552.

276. Kane, A., A. Lau, R. Brink, S. G. Tangye, and E. K. Deenick. 2016. B-cellspecific STAT3 deficiency: Insight into the molecular basis of autosomaldominant hyper-IgE syndrome. Journal of Allergy and Clinical Immunology 138: 1455-1458.e1453.

277. Mogensen, T. H. 2016. Primary Immunodeficiencies with Elevated IgE. International Reviews of Immunology 35: 39-56.

278. Davis, S., J. Schaller, R. Wedgwood, and M. D. Harvard. 1966. JOB'S SYNDROME. The Lancet 287: 1013-1015.

279. Zhang, Q., J. C. Davis, I. T. Lamborn, A. F. Freeman, H. Jing, A. J. Favreau, H. F. Matthews, J. Davis, M. L. Turner, G. Uzel, S. M. Holland, and H. C. Su. 2009. Combined Immunodeficiency Associated with DOCK8 Mutations. New England Journal of Medicine 361: 2046-2055.

280. Minegishi, Y., M. Saito, T. Morio, K. Watanabe, K. Agematsu, S. Tsuchiya, H. Takada, T. Hara, N. Kawamura, T. Ariga, H. Kaneko, N. Kondo, I. Tsuge, A. Yachie, Y. Sakiyama, T. Iwata, F. Bessho, T. Ohishi, K. Joh, K. Imai, K. Kogawa, M. Shinohara, M. Fujieda, H. Wakiguchi, S. Pasic, M. Abinun, H. D. Ochs, E. D. Renner, A. Jansson, B. H. Belohradsky, A. Metin, N. Shimizu, S. Mizutani, T. Miyawaki, S. Nonoyama, and H. Karasuyama. 2006. Human Tyrosine Kinase 2 Deficiency Reveals Its Requisite Roles in Multiple Cytokine Signals Involved in Innate and Acquired Immunity. Immunity 25: 745-755.

281. Zhang, Y., X. Yu, M. Ichikawa, J. J. Lyons, S. Datta, I. T. Lamborn, H. Jing, E. S. Kim, M. Biancalana, L. A. Wolfe, T. DiMaggio, H. F. Matthews, S. M. Kranick, K. D. Stone, S. M. Holland, D. S. Reich, J. D. Hughes, H. Mehmet, J. McElwee, A. F. Freeman, H. H. Freeze, H. C. Su, and J. D. Milner. 2014. Autosomal recessive phosphoglucomutase 3 (<em>PGM3</em>) mutations link glycosylation defects to atopy, immune deficiency, autoimmunity, and neurocognitive impairment. Journal of Allergy and Clinical Immunology 133: 1400-1409.e1405.

282. Sassi, A., S. Lazaroski, G. Wu, S. M. Haslam, M. Fliegauf, F. Mellouli, T. Patiroglu, E. Unal, M. A. Ozdemir, Z. Jouhadi, K. Khadir, L. Ben-Khemis, M. Ben-Ali, I. Ben-Mustapha, L. Borchani, D. Pfeifer, T. Jakob, M. Khemiri, A. C. Asplund, M. O. Gustafsson, K. E. Lundin, E. Falk-Sörqvist, L. N. Moens, H. E. Gungor, K. R. Engelhardt, M. Dziadzio, H. Stauss, B. Fleckenstein, R. Meier, K. Prayitno, A. Maul-Pavicic, S. Schaffer, M. Rakhmanov, P. Henneke, H. Kraus, H. Eibel, U. Kölsch, S. Nadifi, M. Nilsson, M. Bejaoui, A. A. Schäffer, C. I. E. Smith, A. Dell, M.-R. Barbouche, and B. Grimbacher. 2014. Hypomorphic homozygous mutations in phosphoglucomutase 3 (<em>PGM3</em>) impair immunity and increase serum IgE levels. Journal of Allergy and Clinical Immunology 133: 1410-1419.e1413.

283. Mogensen, T. H. 2013. STAT3 and the Hyper-IgE syndrome. JAK-STAT 2: e23435. 
284. Eberting, C. D., J. Davis, J. M. Puck, S. M. Holland, and M. L. Turner. 2004. Dermatitis and the newborn rash of hyper-ige syndrome. Archives of Dermatology 140: 1119-1125.

285. Huang, J. T., M. Abrams, B. Tlougan, A. Rademaker, and A. S. Paller. 2009. Treatment of <em $>$ Staphylococcus aureus $</$ em $>$ Colonization in Atopic Dermatitis Decreases Disease Severity. Pediatrics 123: e808-e814.

286. Grimbacher, B., S. M. Holland, J. I. Gallin, F. Greenberg, S. C. Hill, H. L. Malech, J. A. Miller, A. C. O'Connell, and J. M. Puck. 1999. Hyper-IgE Syndrome with Recurrent Infections - An Autosomal Dominant Multisystem Disorder. New England Journal of Medicine 340: 692-702.

287. Freeman, A. F., J. Davis, V. L. Anderson, W. Barson, D. N. Darnell, J. M. Puck, and S. M. Holland. 2006. \&lt;em\&gt;Pneumocystis jiroveci\&lt;/em\&gt; Infection in Patients With Hyper-Immunoglobulin E Syndrome. Pediatrics 118: e1271.

288. Freeman, A. F., D. E. Kleiner, H. Nadiminti, J. Davis, M. Quezado, V. Anderson, J. M. Puck, and S. M. Holland. 2007. Causes of death in hyper-IgE syndrome. Journal of Allergy and Clinical Immunology 119: 1234-1240.

289. Garty, B. Z., A. Ben-Baruch, A. Rolinsky, C. Woellner, B. Grimbacher, and N. Marcus. 2009. Pneumocystis jirovecii pneumonia in a baby with hyper-IgE syndrome. European Journal of Pediatrics 169: 35.

290. Hutto, J. O. J., C. S. Bryan, F. L. Greene, C. J. White, and J. I. Gallin. Cryptococcosis of the colon resembling Crohn's disease in a patient with the hyperimmunoglobulinemia E-recurrent infection (Job's) syndrome. GASTROENTEROLOGY 94: 808-812.

291. Sowerwine, K. J., S. M. Holland, and A. F. Freeman. 2012. Hyper-IgE syndrome update. Annals of the New York Academy of Sciences 1250: 25-32.

292. Buckley, R. H. 2001. The hyper-IgE syndrome. Clinical Reviews in Allergy \& Immunology 20: 139-154.

293. O’Connell, A. C., J. M. Puck, B. Grimbacher, F. Facchetti, A. Majorana, J. I. Gallin, H. L. Malech, and S. M. Holland. 2000. Delayed eruption of permanent teeth in hyperimmunoglobulinemia E recurrent infection syndrome. Oral Surgery, Oral Medicine, Oral Pathology, Oral Radiology and Endodontics 89: 177-185.

294. Freeman, A. F., E. M. Avila, P. A. Shaw, J. Davis, A. P. Hsu, P. Welch, J. R. Matta, C. Hadigan, R. I. Pettigrew, S. M. Holland, and A. M. Gharib. 2011. Coronary Artery Abnormalities in Hyper-IgE Syndrome. Journal of Clinical Immunology 31: 338-345.

295. Freeman, A. F., C. J. Collura-Burke, N. J. Patronas, L. S. Ilcus, D. Darnell, J. Davis, J. M. Puck, and S. M. Holland. 2007. Brain Abnormalities in Patients With Hyperimmunoglobulin E Syndrome. Pediatrics 119: e1121-e1125.

296. Holland, S. M., F. R. DeLeo, H. Z. Elloumi, A. P. Hsu, G. Uzel, N. Brodsky, A. F. Freeman, A. Demidowich, J. Davis, M. L. Turner, V. L. Anderson, D. N. Darnell, P. A. Welch, D. B. Kuhns, D. M. Frucht, H. L. Malech, J. I. Gallin, S. D. Kobayashi, A. R. Whitney, J. M. Voyich, J. M. Musser, C. Woellner, A. A. Schäffer, J. M. Puck, and B. Grimbacher. 2007. STAT3 Mutations in the HyperIgE Syndrome. New England Journal of Medicine 357: 1608-1619.

297. Minegishi, Y., M. Saito, S. Tsuchiya, I. Tsuge, H. Takada, T. Hara, N. Kawamura, T. Ariga, S. Pasic, O. Stojkovic, A. Metin, and H. Karasuyama. 2007. 
Dominant-negative mutations in the DNA-binding domain of STAT3 cause hyper-IgE syndrome. Nature 448: 1058.

298. Renner, E. D., S. Rylaarsdam, S. Aňover-Sombke, A. L. Rack, J. Reichenbach, J. C. Carey, Q. Zhu, A. F. Jansson, J. Barboza, L. F. Schimke, M. F. Leppert, M. M. Getz, R. A. Seger, H. R. Hill, B. H. Belohradsky, T. R. Torgerson, and H. D. Ochs. 2008. Novel signal transducer and activator of transcription 3 (STAT3) mutations, reduced $\mathrm{T}<\mathrm{sub}>\mathrm{H}</ \mathrm{sub}>17$ cell numbers, and variably defective STAT3 phosphorylation in hyper-IgE syndrome. Journal of Allergy and Clinical Immunology 122: 181-187.

299. Bocchini, C. E., K. Nahmod, P. Katsonis, S. Kim, M. M. Kasembeli, A. Freeman, O. Lichtarge, G. Makedonas, and D. J. Tweardy. 2016. Protein stabilization improves STAT3 function in autosomal dominant hyper-IgE syndrome. Blood 128: 3061-3072.

300. Akira, S., Y. Nishio, M. Inoue, X.-J. Wang, S. We, T. Matsusaka, K. Yoshida, T. Sudo, M. Naruto, and T. Kishimoto. 1994. Molecular cloning of APRF, a novel IFN-stimulated gene factor 3 p91-related transcription factor involved in the gp130-mediated signaling pathway. Cell 77: 63-71.

301. O'Shea, John J., and R. Plenge. 2012. JAK and STAT Signaling Molecules in Immunoregulation and Immune-Mediated Disease. Immunity 36: 542-550.

302. Harrison, D. A. 2012. The JAK/STAT Pathway. Cold Spring Harbor Perspectives in Biology 4.

303. Rawlings, J. S., K. M. Rosler, and D. A. Harrison. 2004. The JAK/STAT signaling pathway. Journal of Cell Science 117: 1281-1283.

304. Wang, W.-B., D. E. Levy, and C.-K. Lee. 2011. STAT3 Negatively Regulates Type I IFN-Mediated Antiviral Response. The Journal of Immunology 187: 25782585.

305. Puel, A., S. Cypowyj, L. Maródi, L. Abel, C. Picard, and J.-L. Casanova. 2012. Inborn errors of human IL-17 immunity underlie chronic mucocutaneous candidiasis. Current Opinion in Allergy and Clinical Immunology 12: 616-622.

306. El Kasmi, K. C., J. Holst, M. Coffre, L. Mielke, A. de Pauw, N. Lhocine, A. M. Smith, R. Rutschman, D. Kaushal, Y. Shen, T. Suda, R. P. Donnelly, M. G. Myers, Jr., W. Alexander, D. A. Vignali, S. S. Watowich, M. Ernst, D. J. Hilton, and P. J. Murray. 2006. General nature of the STAT3-activated anti-inflammatory response. J Immunol 177: 7880-7888.

307. Hodge, D. R., E. M. Hurt, and W. L. Farrar. 2005. The role of IL-6 and STAT3 in inflammation and cancer. European Journal of Cancer 41: 2502-2512.

308. He, J., J. Shi, X. Xu, W. Zhang, Y. Wang, X. Chen, Y. Du, N. Zhu, J. Zhang, Q. Wang, and J. Yang. 2012. STAT3 mutations correlated with hyper-IgE syndrome lead to blockage of IL-6/STAT3 signalling pathway. J Biosci 37: 243-257.

309. Sonnenberg, G. F., L. A. Fouser, and D. Artis. 2011. Border patrol: regulation of immunity, inflammation and tissue homeostasis at barrier surfaces by IL-22. Nature Immunology 12: 383.

310. Hill, H., P. Quie, H. Pabst, H. Ochs, R. Clark, S. Klebanoff, and R. Wedgwood. 1974. DEFECT IN NEUTROPHIL GRANULOCYTE CHEMOTAXIS IN JOB'S SYNDROME OF RECURRENT "COLD" STAPHYLOCOCCAL ABSCESSES. The Lancet 304: 617-619. 
311. Milner, J. D., J. M. Brenchley, A. Laurence, A. F. Freeman, B. J. Hill, K. M. Elias, Y. Kanno, C. Spalding, H. Z. Elloumi, M. L. Paulson, J. Davis, A. Hsu, A. I. Asher, J. O'Shea, S. M. Holland, W. E. Paul, and D. C. Douek. 2008. Impaired TH17 cell differentiation in subjects with autosomal dominant hyper-IgE syndrome. Nature 452: 773.

312. Tamassia, N., M. Castellucci, M. Rossato, S. Gasperini, D. Bosisio, M. Giacomelli, R. Badolato, M. A. Cassatella, and F. Bazzoni. 2009. Uncovering an IL-10-dependent NF- $\kappa \mathrm{B}$ recruitment to the IL-1ra promoter that is impaired in STAT3 functionally defective patients. The FASEB Journal 24: 1365-1375.

313. Speckmann, C., A. Enders, C. Woellner, D. Thiel, A. Rensing-Ehl, M. Schlesier, J. Rohr, T. Jakob, E. Oswald, M. V. Kopp, O. Sanal, J. Litzman, A. Plebani, M. C. Pietrogrande, J. L. Franco, T. Espanol, B. Grimbacher, and S. Ehl. 2008. Reduced memory B cells in patients with hyper IgE syndrome. Clinical Immunology 129: 448-454.

314. Buckley, R. H., and W. G. Becker. 1978. Abnormalities in the Regulation of Human IgE Synthesis. Immunological Reviews 41: 288-314.

315. Ozaki, K., R. Spolski, C. G. Feng, C.-F. Qi, J. Cheng, A. Sher, H. C. Morse, C. Liu, P. L. Schwartzberg, and W. J. Leonard. 2002. A Critical Role for IL-21 in Regulating Immunoglobulin Production. Science 298: 1630-1634.

316. Avery, D. T., C. S. Ma, V. L. Bryant, B. Santner-Nanan, R. Nanan, M. Wong, D. A. Fulcher, M. C. Cook, and S. G. Tangye. 2008. STAT3 is required for IL-21induced secretion of IgE from human naive B cells. Blood 112: 1784-1793.

317. Bassing, C. H., W. Swat, and F. W. Alt. 2002. The Mechanism and Regulation of Chromosomal V(D)J Recombination. Cell 109: S45-S55.

318. Methot, S. P., and J. M. Di Noia. 2017. Chapter Two - Molecular Mechanisms of Somatic Hypermutation and Class Switch Recombination. In Advances in Immunology. F. W. Alt, ed. Academic Press. 37-87.

319. Fitzsimmons, C. M., F. H. Falcone, and D. W. Dunne. 2014. Helminth Allergens, Parasite-Specific IgE, and Its Protective Role in Human Immunity. Frontiers in Immunology 5: 61.

320. Deo, S. S., K. J. Mistry, A. M. Kakade, and P. V. Niphadkar. 2010. Role played by Th2 type cytokines in IgE mediated allergy and asthma. Lung India : Official Organ of Indian Chest Society 27: 66-71.

321. Schedel, M., R. Frei, C. Bieli, L. Cameron, J. Adamski, R. Lauener, and M. Kabesch. 2009. An IgE-associated polymorphism in <em>STAT6</em> alters NF-\&\#x3ba;B binding, <em>STAT6</em> promoter activity, and mRNA expression. Journal of Allergy and Clinical Immunology 124: 583-589.e586.

322. Sugai, M., H. Gonda, T. Kusunoki, T. Katakai, Y. Yokota, and A. Shimizu. 2002. Essential role of Id 2 in negative regulation of IgE class switching. Nature Immunology 4: 25.

323. Zhang, T.-t., K. J. Makondo, and A. J. Marshall. 2012. p1108 Phosphoinositide 3Kinase Represses IgE Switch by Potentiating BCL6 Expression. The Journal of Immunology.

324. Lorentz, A., I. Klopp, T. Gebhardt, M. P. Manns, and S. C. Bischoff. 2003. Role of activator protein 1, nuclear factor-kappaB, and nuclear factor of activated $\mathrm{T}$ 
cells in IgE receptor-mediated cytokine expression in mature human mast cells. $J$ Allergy Clin Immunol 111: 1062-1068.

325. Kashiwada, M., D. M. Levy, L. McKeag, K. Murray, A. J. Schröder, S. M. Canfield, G. Traver, and P. B. Rothman. 2010. IL-4-induced transcription factor NFIL3/E4BP4 controls IgE class switching. Proceedings of the National Academy of Sciences of the United States of America 107: 821-826.

326. Mandler, R., F. D. Finkelman, A. D. Levine, and C. M. Snapper. 1993. IL-4 induction of IgE class switching by lipopolysaccharide-activated murine B cells occurs predominantly through sequential switching. The Journal of Immunology 150: 407-418.

327. Xiong, H., J. Dolpady, M. Wabl, M. A. Curotto de Lafaille, and J. J. Lafaille. 2012. Sequential class switching is required for the generation of high affinity $\operatorname{IgE}$ antibodies. The Journal of Experimental Medicine 209: 353-364.

328. Bolli, R., A. B. Stein, Y. Guo, O. L. Wang, G. Rokosh, B. Dawn, J. D. Molkentin, S. K. Sanganalmath, Y. Zhu, and Y. T. Xuan. 2011. A murine model of inducible, cardiac-specific deletion of STAT3: its use to determine the role of STAT3 in the upregulation of cardioprotective proteins by ischemic preconditioning. $\mathrm{J} \mathrm{Mol} \mathrm{Cell}$ Cardiol 50: 589-597.

329. Tripathi, S. K., Z. Chen, A. Larjo, K. Kanduri, K. Nousiainen, T. Aijo, I. RicanoPonce, B. Hrdlickova, S. Tuomela, E. Laajala, V. Salo, V. Kumar, C. Wijmenga, H. Lahdesmaki, and R. Lahesmaa. 2017. Genome-wide Analysis of STAT3Mediated Transcription during Early Human Th17 Cell Differentiation. Cell Rep 19: 1888-1901.

330. Ding, C., X. Chen, P. Dascani, X. Hu, R. Bolli, H. G. Zhang, K. R. McLeish, and J. Yan. 2016. STAT3 Signaling in B Cells Is Critical for Germinal Center Maintenance and Contributes to the Pathogenesis of Murine Models of Lupus. $J$ Immunol 196: 4477-4486.

331. Kerfoot, S. M., G. Yaari, J. R. Patel, K. L. Johnson, D. G. Gonzalez, S. H. Kleinstein, and A. M. Haberman. 2011. Germinal center B cell and T follicular helper cell development initiates in the inter-follicular zone. Immunity 34: 947960.

332. O'Connor, B. P., L. A. Vogel, W. Zhang, W. Loo, D. Shnider, E. F. Lind, M. Ratliff, R. J. Noelle, and L. D. Erickson. 2006. Imprinting the fate of antigenreactive B cells through the affinity of the B cell receptor. J Immunol 177: 77237732.

333. Victora, G. D., and M. C. Nussenzweig. 2012. Germinal Centers. Annual Review of Immunology 30: 429-457.

334. De Silva, N. S., and U. Klein. 2015. Dynamics of B cells in germinal centres. Nature reviews. Immunology 15: 137-148.

335. Sander, S., V. T. Chu, T. Yasuda, A. Franklin, R. Graf, D. P. Calado, S. Li, K. Imami, M. Selbach, M. Di Virgilio, L. Bullinger, and K. Rajewsky. 2015. PI3 Kinase and FOXO1 Transcription Factor Activity Differentially Control B Cells in the Germinal Center Light and Dark Zones. Immunity 43: 1075-1086.

336. Hamel, K. M., V. M. Liarski, and M. R. Clark. 2012. Germinal center B-cells. Autoimmunity 45: 333-347. 
337. Basso, K., C. Schneider, Q. Shen, A. B. Holmes, M. Setty, C. Leslie, and R. Dalla-Favera. 2012. BCL6 positively regulates AID and germinal center gene expression via repression of miR-155. J Exp Med 209: 2455-2465.

338. Lien, C., C.-M. Fang, D. Huso, F. Livak, R. Lu, and P. M. Pitha. 2010. Critical role of IRF-5 in regulation of B-cell differentiation. Proceedings of the National Academy of Sciences 107: 4664-4668.

339. Zheng, Y., A. Chaudhry, A. Kas, P. deRoos, J. M. Kim, T. T. Chu, L. Corcoran, P. Treuting, U. Klein, and A. Y. Rudensky. 2009. Regulatory T-cell suppressor program co-opts transcription factor IRF4 to control T(H)2 responses. Nature 458: 351-356.

340. Froidure, A., J. Mouthuy, S. R. Durham, P. Chanez, Y. Sibille, and C. Pilette. 2016. Asthma phenotypes and IgE responses. European Respiratory Journal 47: 304-319.

341. Turqueti-Neves, A., M. Otte, O. P. da Costa, U. E. Höpken, M. Lipp, T. Buch, and D. Voehringer. 2014. B-cell-intrinsic STAT6 signaling controls germinal center formation. European Journal of Immunology 44: 2130-2138.

342. Fornek, J. L., L. T. Tygrett, T. J. Waldschmidt, V. Poli, R. C. Rickert, and G. S. Kansas. 2006. Critical role for Stat3 in T-dependent terminal differentiation of IgG B cells. Blood 107: 1085-1091.

343. Kitayama, D., A. Sakamoto, M. Arima, M. Hatano, M. Miyazaki, and T.

Tokuhisa. 2008. A role for Bcl6 in sequential class switch recombination to $\operatorname{IgE}$ in B cells stimulated with IL-4 and IL-21. Molecular Immunology 45: 1337-1345.

344. Misaghi, S., K. Senger, T. Sai, Y. Qu, Y. Sun, K. Hamidzadeh, A. Nguyen, Z. Jin, M. Zhou, D. Yan, W. Y. Lin, Z. Lin, M. N. Lorenzo, A. Sebrell, J. Ding, M. Xu, P. Caplazi, C. D. Austin, M. Balazs, M. Roose-Girma, L. DeForge, S. Warming, W. P. Lee, V. M. Dixit, and A. A. Zarrin. 2013. Polyclonal hyper-IgE mouse model reveals mechanistic insights into antibody class switch recombination. Proceedings of the National Academy of Sciences of the United States of America 110: 15770-15775.

345. Leonard, W., and C. Wan. 2016. IL-21 Signaling in Immunity [version 1; referees: 3 approved].

346. Choi, Y. S., J. A. Yang, and S. Crotty. 2013. Dynamic regulation of Bc16 in follicular helper CD4 T (Tfh) cells. Current Opinion in Immunology 25: 366-372.

347. Sander, S., Van T. Chu, T. Yasuda, A. Franklin, R. Graf, Dinis P. Calado, S. Li, K. Imami, M. Selbach, M. Di Virgilio, L. Bullinger, and K. Rajewsky. PI3 Kinase and FOXO1 Transcription Factor Activity Differentially Control B Cells in the Germinal Center Light and Dark Zones. Immunity 43: 1075-1086.

348. Beltman, J. B., C. D. C. Allen, J. G. Cyster, and R. J. de Boer. 2011. B cells within germinal centers migrate preferentially from dark to light zone.

Proceedings of the National Academy of Sciences 108: 8755-8760.

349. Oh, H.-M., C.-R. Yu, N. Golestaneh, A. Amadi-Obi, Y. S. Lee, A. Eseonu, R. M. Mahdi, and C. E. Egwuagu. 2011. STAT3 Protein Promotes T-cell Survival and Inhibits Interleukin-2 Production through Up-regulation of Class O Forkhead Transcription Factors. Journal of Biological Chemistry 286: 30888-30897.

350. Zhang, K., F. C. Mills, and A. Saxon. 1994. Switch circles from IL-4-directed epsilon class switching from human B lymphocytes. Evidence for direct, 
sequential, and multiple step sequential switch from mu to epsilon Ig heavy chain gene. J Immunol 152: 3427-3435.

351. Cameron, L., A. S. Gounni, S. Frenkiel, F. Lavigne, D. Vercelli, and Q. Hamid. 2003. $\mathrm{S} \varepsilon S \mu$ and $\mathrm{S} \varepsilon S \gamma$ Switch Circles in Human Nasal Mucosa Following Ex Vivo Allergen Challenge: Evidence for Direct as Well as Sequential Class Switch Recombination. The Journal of Immunology 171: 3816-3822.

352. Hebenstreit, D., G. Wirnsberger, J. Horejs-Hoeck, and A. Duschl. 2006. Signaling mechanisms, interaction partners, and target genes of STAT6. Cytokine Growth Factor Rev 17: 173-188.

353. Harris, M. B., C. C. Chang, M. T. Berton, N. N. Danial, J. Zhang, D. Kuehner, B. H. Ye, M. Kvatyuk, P. P. Pandolfi, G. Cattoretti, R. Dalla-Favera, and P. B. Rothman. 1999. Transcriptional repression of Stat6-dependent interleukin-4induced genes by BCL-6: specific regulation of iepsilon transcription and immunoglobulin E switching. Mol Cell Biol 19: 7264-7275.

354. Audzevich, T., G. Pearce, M. Breucha, G. Günal, and R. Jessberger. 2013. Control of the STAT6-BCL6 Antagonism by SWAP-70 Determines IgE Production. The Journal of Immunology 190: 4946-4955.

355. Walker, S. R., E. A. Nelson, J. E. Yeh, L. Pinello, G.-C. Yuan, and D. A. Frank. 2013. STAT5 Outcompetes STAT3 To Regulate the Expression of the Oncogenic Transcriptional Modulator BCL6. Molecular and Cellular Biology 33: 2879-2890.

356. Arguni, E., M. Arima, N. Tsuruoka, A. Sakamoto, M. Hatano, and T. Tokuhisa. 2006. JunD/AP-1 and STAT3 are the major enhancer molecules for high Bcl6 expression in germinal center B cells. Int Immunol 18: 1079-1089.

357. Ochiai, K., M. Maienschein-Cline, G. Simonetti, J. Chen, R. Rosenthal, R. Brink, A. S. Chong, U. Klein, A. R. Dinner, H. Singh, and R. Sciammas. 2013. Transcriptional regulation of germinal center B and plasma cell fates by dynamical control of IRF4. Immunity 38: 918-929.

358. Diehl, S. A., H. Schmidlin, M. Nagasawa, S. D. van Haren, M. J. Kwakkenbos, E. Yasuda, T. Beaumont, F. A. Scheeren, and H. Spits. 2008. STAT3-Mediated UpRegulation of BLIMP1 Is Coordinated with BCL6 Down-Regulation to Control Human Plasma Cell Differentiation. Journal of immunology (Baltimore, Md. : 1950) 180: 4805-4815.

359. Tong, P., and D. R. Wesemann. 2015. Molecular Mechanisms of IgE Class Switch Recombination. Curr Top Microbiol Immunol 388: 21-37.

360. Sherman, M. H., A. I. Kuraishy, C. Deshpande, J. S. Hong, N. A. Cacalano, R. A. Gatti, J. P. Manis, M. A. Damore, M. Pellegrini, and M. A. Teitell. 2010. AIDInduced Genotoxic Stress Promotes B Cell Differentiation in the Germinal Center via ATM and LKB1 Signaling. Molecular cell 39: 873-885.

361. Walsh, N. C., L. R. Waters, J. A. Fowler, M. Lin, C. R. Cunningham, D. G. Brooks, J. E. Rehg, H. C. Morse, and M. A. Teitell. 2015. LKB1 inhibition of NF$\kappa \mathrm{B}$ in $\mathrm{B}$ cells prevents $\mathrm{T}$ follicular helper cell differentiation and germinal center formation. EMBO Reports 16: 753-768.

362. Heimall, J., A. Freeman, and S. M. Holland. 2010. Pathogenesis of Hyper IgE Syndrome. Clinical Reviews in Allergy \& Immunology 38: 32-38.

363. Massaad, M. J., B. Cangemi, W. Al-Herz, G. LeFranc, A. Freeman, S. Baxi, S. Keles, A. Metin, M. Dasouki, A. Sobh, M. Kanariou, N. Al-Sukaiti, A. Ozen, H. 
Ochs, T. A. Chatila, J. P. Manis, and R. Geha. 2017. DOCK8 and STAT3 dependent inhibition of IgE isotype switching by TLR9 ligation in human B cells. Clinical Immunology 183: 263-265.

364. SCHULMAN, E. S. 2001. Development of a Monoclonal Anti-Immunoglobulin E Antibody (Omalizumab) for the Treatment of Allergic Respiratory Disorders. American Journal of Respiratory and Critical Care Medicine 164: S6-S11.

365. Siegel, A. M., K. D. Stone, G. Cruse, M. G. Lawrence, A. Olivera, M.-y. Jung, J. S. Barber, A. F. Freeman, S. M. Holland, M. O'Brien, N. Jones, C. G. Nelson, L. B. Wisch, H. H. Kong, A. Desai, O. Farber, A. M. Gilfillan, J. Rivera, and J. D. Milner. 2013. Diminished allergic disease in patients with STAT3 mutations reveals a role for STAT3 signaling in mast cell degranulation. The Journal of allergy and clinical immunology 132: 1388-1396.

366. Gavino, A. C., K. Nahmod, U. Bharadwaj, G. Makedonas, and D. J. Tweardy. 2016. STAT3 inhibition prevents lung inflammation, remodeling, and accumulation of Th2 and Th17 cells in a murine asthma model. Allergy 71: 16841692.

367. Grimbacher, B., S. M. Holland, J. I. Gallin, F. Greenberg, S. C. Hill , H. L. Malech , J. A. Miller, A. C. O'Connell, and J. M. Puck 1999. Hyper-IgE Syndrome with Recurrent Infections - An Autosomal Dominant Multisystem Disorder. New England Journal of Medicine 340: 692-702.

368. Steward-Tharp, S. M., A. Laurence, Y. Kanno, A. Kotlyar, A. V. Villarino, G. Sciume, S. Kuchen, W. Resch, E. A. Wohlfert, K. Jiang, K. Hirahara, G. Vahedi, H.-w. Sun, L. Feigenbaum, J. D. Milner, S. M. Holland, R. Casellas, F. Powrie, and J. J. O'Shea. 2014. A mouse model of HIES reveals pro- and antiinflammatory functions of STAT3. Blood 123: 2978-2987. 


\section{CURRICULUM VITAE \\ Paul Dascani, Ph.D. \\ Tel: 724-244-8943 \\ pdascani@gmail.com}

\section{EDUCATION}

2013-2018 PhD in Immunology, University of Louisville, Louisville KY

2013-2015 MSc. in Immunology, University of Louisville, Louisville KY

2007-2011 BSc in Biology, University of Pittsburgh, Pittsburgh PA

\section{OTHER POSITIONS AND EMPLOYMENT}

2011-2013 Research Technician, Division of Immunogenetics, University of

Pittsburgh, Pittsburgh PA

\section{PROFESSIONAL MEMBERSHIPS AND ACTIVITIES}

2016-2018 Policy Coordinator and President, University of Louisville Science Policy and Outreach Group (SPOG)

2016-2018 Student Member, American Association of Immunologists (AAI)

\section{EDUCATIONAL ACTIVITIES}

2017-2018 Graduate Student Mentor, University of Louisville, Louisville KY

2017 Louisville Science Pathways (LSP) Internship Student Mentor,

Louisville, KY

\section{PUBLICATIONS}

ORAL PRESENTATIONS

"Transcription Factor STAT3 Serves as a Negative Regulator Controlling IgE Class Switching in Mice" American Association of Immunologists Annual Meeting 2018, Austin TX

\section{POSTERS}

"Transcription Factor STAT3 Serves as a Negative Regulator Controlling IgE Class Switching in Mice" American Association of Immunologists Annual Meeting 2018, Austin TX 


\section{PEER-REVIEWED/ORIGINAL RESEARCH MANUSCRIPTS}

1. Chuanlin Ding, Xingguo Chen, Paul Dascani, Xiaoling Hu, Roberto Bolli, Huang-ge Zhang, Kenneth R. Mcleish and Jun Yan. "STAT3 Signaling in B Cells Is Critical for Germinal Center Maintenance and Contributes to the Pathogenesis of Murine Models of Lupus" J Immunol, 2016

2. Paul Dascani, Chuanlin Ding, Xiangyu Kong, David Tieri, Xiaoling Hu, Huang-ge Zhang, Daisuke Kitamura, Roberto Bolli, Eric C. Rouchka, Jun Yan. "Transcription

Factor STAT3 Serves as a Negative Regulator Controlling IgE Class Switching in Mice" ImmunoHorizons, 2018

\section{LABORATORY SKILLS}

In vivo main skills:

- Mice: Maintaining mice colonies, breeding, genotyping, intraperitoneal, footpad, and subcutaneous injection, blood and organ collection

- In vivo disease monitoring

Expert at:

- Flow cytometry

- Imaging flow cytometry

- Cell sorting from tissues and cell lines: FACS sorting, magnetic beads cell sorting

- Cell culture: culture of primary cells, cell lines

- Processing of human blood and for FACS analysis

- DNA and RNA isolation: PCR and quantitative RT-PCR

- ELISA (enzyme-linked immunosorbent assay)

- Lymphocyte proliferation assays, B cell differentiation assays

Competent at:

- Confocal microscopy

- Chromatin immunoprecipitation

- Tissue sectioning and preparation

Instruments used:

- Flow cytometry: FACS Calibur, FACS Canto II

- Cell sorting: FACS Aria III, AutoMacs Cell Sorter

- Imaging flow cytometry: Amnis Imagestream X 